\title{
MUON NEUTRINO DISAPPEARANCE AT MINOS
}

Robert E. Armstrong

Submitted to the faculty of the University Graduate School

in partial fulfillment of the requirements

for the degree

Doctor of Philosophy

in the Department of Physics,

Indiana University

August 2009 
Accepted by the Graduate Faculty, Indiana University, in partial fulfillment of the requirements for the degree of Doctor of Philosophy.

Doctoral Committee

Jon Urheim, Ph.D.

Rex Tayloe, Ph.D.

Charles J. Horowitz, Ph.D.

Stuart L. Mufson, Ph.D.

July 31, 2009 
(c) (2009)

Robert E. Armstrong

ALL RIGHTS RESERVED 
For my family,

thanks for everything 


\section{Robert E. Armstrong}

\section{MUON NEUTRINO DISAPPEARANCE AT MINOS}

A strong case has been made by several experiments that neutrinos oscillate, although important questions remain as to the mechanisms and precise values of the parameters. In the standard picture, two parameters describe the nature of how the neutrinos oscillate: the mass-squared difference between states and the mixing angle. The purpose of this thesis is to use data from the MINOS experiment to precisely measure the parameters associated with oscillations first observed in studies of atmospheric neutrinos. MINOS utilizes two similar detectors to observe the oscillatory nature of neutrinos. The Near Detector, located $1 \mathrm{~km}$ from the source, observes the unoscillated energy spectrum while the Far Detector, located $735 \mathrm{~km}$ away, is positioned to see the oscillation signal. Using the data in the Near Detector, a prediction of the expected neutrino spectrum at the Far Detector assuming no oscillations is made. By comparing this prediction with the MINOS data, the atmospheric mixing parameters are measured to be $\Delta m_{32}^{2}=2.45_{+0.12}^{-0.12} \times 10^{-3} \mathrm{eV}^{2}$ and $\sin ^{2}\left(2 \theta_{32}\right)=1.00_{-0.04}^{+0.00}$ ( $>0.90$ at $90 \%$ confidence level). 


\section{Contents}

1 Introduction 1

1.1 Measuring the Atmospheric Oscillation Parameters . . . . . . . . . . . 2

2 Background 5

2.1 The Early Years of Neutrinos . . . . . . . . . . . . . . . 5

2.2 Neutrino Mixing . . . . . . . . . . . . . . . . . . 7

2.3 Neutrino Interactions . . . . . . . . . . . . . . . . . . . . . 11

2.4 Solar Neutrino Problem . . . . . . . . . . . . . . . . . . . . . . 13

2.5 Atmospheric Neutrino Problem . . . . . . . . . . . . . . . . . . . 14

3 The NuMI Beam and the MINOS Detectors 20

3.1 The NuMI Beam . . . . . . . . . . . . . . . . . . . . . . . 20

3.1 .1 Proton Beam . . . . . . . . . . . . . . . . 2 20

3.1 .2 NuMI Target . . . . . . . . . . . . . . . . . 21

3.1 .3 Focusing Horns . . . . . . . . . . . . . . . . . . . . . 23

3.1 .4 Decay Volume . . . . . . . . . . . . . . . . . . . . . . 24

3.1.5 Different Beam Configurations . . . . . . . . . . . . 26

3.1.6 Uncertainties on the $\nu_{\mu}$ Flux . . . . . . . . . . . . . . 34

3.2 The MINOS Detectors . . . . . . . . . . . . . . . . . . 34

3.2 .1 Near Detector . . . . . . . . . . . . . . . . . . . 36

3.2 .2 Far Detector . . . . . . . . . . . . . . . . . . 39

3.2 .3 Calibration Detector . . . . . . . . . . . . . . . 41

4 Detector Operations and Simulations 43

4.1 Reconstruction Algorithms . . . . . . . . . . . . . . . . . . 43

4.2 Intensity Dependence of the Near Detector Data . . . . . . . . . . . . . 46

4.3 Monte Carlo Simulation . . . . . . . . . . . . . . . . . . . . . 47

4.3.1 Interactions in the Target . . . . . . . . . . . . . . 48

4.3.2 Propagation Through the NuMi Beamline . . . . . . . . . . . 48 
4.3.3 Neutrino Interactions in the MINOS Detectors . . . . . . . . . . . 49

4.3.4 Modeling Neutrino Interactions with NEUGEN . . . . . . . . . . 49

4.3.5 Light Transport . . . . . . . . . . . . . . . . . . . . 49

5 Hadron Production Tuning $\quad 51$

5.1 Existing Hadron Production Data . . . . . . . . . . . . . . . . 52

5.2 Hadron Production Parameterization . . . . . . . . . . . . . . 54

5.3 Constraining the Monte Carlo: The Fit to the Near Detector Data . . . . 57

5.3.1 Hadron Production Parameters . . . . . . . . . . . . . . 59

5.3 .2 Beam Parameters . . . . . . . . . . . . . . . 61

5.3 .3 Detector Parameters . . . . . . . . . . . . . . . 62

5.4 Data Sets . . . . . . . . . . . . . . . . . . . . 64

5.5 Smearing . . . . . . . . . . . . . . . . . 66

5.6 Fit Results . . . . . . . . . . . . . . . . 67

5.6 .1 Comments . . . . . . . . . . . . . . . 77

5.6.2 Effect on Hadron Production . . . . . . . . . . . . . . . . 77

5.6.3 Comparison to External Data . . . . . . . . . . . . . . . 80

5.7 Uncertainty on the Flux after the Fit . . . . . . . . . . . . 83

5.7.1 Uncertainty from Hadron Production after the Fit . . . . . . . . 83

5.7 .2 Total Uncertainty . . . . . . . . . . . . . . . . . . 84

5.7 .3 Correlations on the Fit Parameters . . . . . . . . . . . . . 84

5.7 .4 Impact on the Flux . . . . . . . . . . . . . . . 84

6 Near Detector Data and Extrapolation to the Far Detector $\quad 91$

6.1 Event Selection and Data/MC comparisons . . . . . . . . . . . . . 91

6.1.1 Pre-Selection Cuts . . . . . . . . . . . . . . . . . . 91

6.1.2 Distributions Studied . . . . . . . . . . . . . . . . . . 93

6.1.3 LE RunI Data/MC Comparison . . . . . . . . . . . . . . . . . 93

6.1.4 LE RunII Data/MC Comparison . . . . . . . . . . . . . . . . 100

6.1.5 HE RunII Data/MC Comparison . . . . . . . . . . . . . . . . . 107

6.1.6 Comments . . . . . . . . . . . . . . . . . . 114

6.2 Extrapolation to the Far Detector . . . . . . . . . . . . . . . . . . 114

6.2.1 Reweighting . . . . . . . . . . . . . . 117

7 Selecting Charged Current Events 118

7.1 Charged Current Events . . . . . . . . . . . . . . . . . . 118

$7.2 \mathrm{kNN}$ Method . . . . . . . . . . . . . . . . . . . . 119

7.3 Likelihood or PDF Method . . . . . . . . . . . . . . . . . . . . 121 
7.4 ANN Method . . . . . . . . . . . . . . . . . . . . . 122

7.5 A Comparison of Selection Algorithms . . . . . . . . . . . . . . 128

7.6 Improvements Over the Standard Implementation . . . . . . . . . . . . 132

8 Systematic Uncertainties $\quad 134$

8.1 Beam Related Uncertainties . . . . . . . . . . . . . . . . . . . . . 134

8.1.1 Geomagnetic Field in the Decay Pipe . . . . . . . . . . . . . . . 134

8.1.2 Time Dependent Change to the Flux . . . . . . . . . . . . . . . . 139

8.2 Relative Normalization . . . . . . . . . . . . . . . . . . . . . . . . . 142

8.3 Hadronic Energy Scale . . . . . . . . . . . . . . . . . . . . 143

8.3.1 Absolute Energy Scale . . . . . . . . . . . . . . . . . . 144

8.3.2 Final State Uncertainties . . . . . . . . . . . . . . . . . . . . 144

8.3.3 Relative Uncertainty . . . . . . . . . . . . . . . . . . . . . . 144

8.4 Neutral Current Contamination . . . . . . . . . . . . . . . . . 145

8.5 Muon Momentum . . . . . . . . . . . . . . . . . . . . . . . . . . . 147

8.6 Cross-Section . . . . . . . . . . . . . . . . . . . 147

8.7 Flux . . . . . . . . . . . . . . . . . . . . . 148

8.8 Extrapolation Errors . . . . . . . . . . . . . . . . . . . . . . . . . . 148

8.9 Residual Difference from Near Detector Fit . . . . . . . . . . . . . . . . . 149

8.10 Impact on the Oscillation Measurements . . . . . . . . . . . . . . . . . 149

8.10 .1 Global Errors . . . . . . . . . . . . . . . . . . . . . 149

8.10 .2 Relative Errors . . . . . . . . . . . . . . . . . . 158

8.11 Discussion . . . . . . . . . . . . . . . . . . . 160

9 Analysis of the Far Detector Data $\quad 161$

9.1 Selection Cuts . . . . . . . . . . . . . . . . . . . 162

9.2 Blinding . . . . . . . . . . . . . . . . . . 165

9.3 Data in the Far Detector . . . . . . . . . . . . . . . . . . . 165

9.4 Oscillation Analysis . . . . . . . . . . . . . . . . . . . . . 174

9.4.1 Fit to Individual Runs . . . . . . . . . . . . . . . . . . . . 181

9.4.2 Fitting the Data with No Cuts . . . . . . . . . . . . 183

9.4.3 Fitting with Systematics . . . . . . . . . . . . . . . . 183

9.5 Comparison with Published MINOS Results . . . . . . . . . . . . . . . 184

9.6 Dip at $5 \mathrm{GeV} \ldots \ldots \ldots \ldots \ldots$. . . . . . . . . . . . . . . 184

9.6.1 Cut Relaxation . . . . . . . . . . . . . . . 186

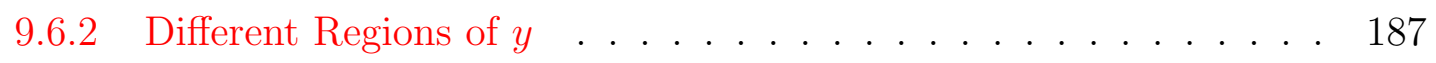

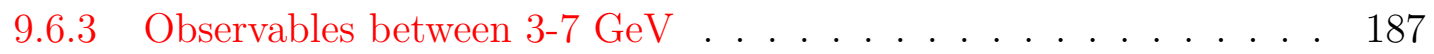


9.6.4 Focusing Effects . . . . . . . . . . . . . . . . . . . . . . . 190

9.6.5 Summary of $5 \mathrm{GeV}$ dip . . . . . . . . . . . . . . . . . 190

9.7 Alternatives to Neutrino Oscillations . . . . . . . . . . . . . . . . . 192

9.7.1 Neutrino Decay . . . . . . . . . . . . . . . . . . . . . . . . 192

9.7 .2 Neutrino Decoherence . . . . . . . . . . . . . . . . . . 193

9.7.3 Lorentz and CPT Invariance Violation . . . . . . . . . . . . . . 193

9.8 Measurements at a Boundary . . . . . . . . . . . . . . . . . 196

9.9 Future Sensitivity . . . . . . . . . . . . . . . . . . . . . . . 198

10 Summary and Outlook 205

$\begin{array}{ll}\text { A Uncertainties to the } \nu_{e} \text { Flux } & 209\end{array}$

A.1 Analysis Procedure . . . . . . . . . . . . . . . . . . . 211

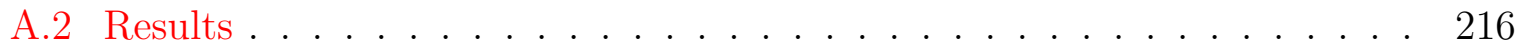

$\begin{array}{lr}\text { Bibliography } & 217\end{array}$ 


\section{List of Figures}

$2.1 \mathrm{CC}$ and $\mathrm{NC}$ interactions. . . . . . . . . . . . . . 11

2.2 Different types of $\mathrm{CC}$ interactions. . . . . . . . . . . . . . . 13

2.3 Solar neutrinos measured by SNO . . . . . . . . . . . . . . 15

2.4 Measurements from KamLAND and solar neutrino experiments. . . . . 16

2.5 Zenith angle distributions from SuperKamiokande. . . . . . . . . . . . 17

2.6 Neutrino oscillations as a function of $L / E$ for SuperKamiokande. . . . . 19

2.7 Neutrino energy spectrum from K2K. . . . . . . . . . . . . . . 19

3.1 The layout of the NuMI beamline. . . . . . . . . . . . . . . . 21

3.2 The NuMI target. . . . . . . . . . . . . . . . . . 22

3.3 Horn 1 of the NuMI beamline. . . . . . . . . . . . . . . 23

3.4 Horn 2 of the NuMI beamline. . . . . . . . . . . . . . . . 24

3.5 Possible trajectories for hadrons in the NuMI beam. . . . . . . . . . . 25

3.6 Probability for a $\pi^{+}$to interact for different beam configurations. . . . . 27

3.7 Probability for a $\pi^{-}$to interact for different beam configurations. . . . . 28

3.8 Probability for a $K^{+}$to interact for different beam configurations. . . . 29

3.9 Probability for a $K^{-}$to interact for different beam configurations. . . . 30

3.10 Neutrino energy spectrum for different beam configurations. . . . . . . . 31

$3.11 \nu_{\mu}$ energy spectrum from different parents. . . . . . . . . . . . . 32

$3.12 \bar{\nu}_{\mu}$ energy spectrum from different parents. . . . . . . . . . . . 33

3.13 The flux uncertainties on the $\nu_{\mu}$ flux. . . . . . . . . . . . 35

3.14 The scintillator readout system. . . . . . . . . . . . . . . 35

3.15 The MINOS Near Detector. . . . . . . . . . . . . . . . . 37

3.16 Different scintillator modules. . . . . . . . . . . . . . . . 37

3.17 A beam spill in the Near Detector. . . . . . . . . . . . . . . . 38

3.18 The MINOS Far Detector. . . . . . . . . . . . . . . . 40

3.19 Calorimetric response of the Calibration Detector. . . . . . . . . . . 42

4.1 Accumulated POT from the NuMI beamline. . . . . . . . . . . . . . 44

$4.2 \mathrm{CC}$ and $\mathrm{NC}$ events in MINOS. . . . . . . . . . . . . . 45 
4.3 Neutrino events as function of beam intensity. . . . . . . . . . . . . 47

4.4 Neutrino cross-section measurements. . . . . . . . . . . . . . . . . 50

5.1 External hadron production data. . . . . . . . . . . . . . . 53

5.2 Different model predictions for the MINOS neutrino flux. . . . . . . . 55

5.3 Hadron production as a function of $x_{F}$ from FLUKA. . . . . . . . 57

5.4 Fit coefficients as a function of $x_{F} \ldots \ldots \ldots \ldots \ldots$

5.5 The effect of changing cross-section parameters on the $\nu_{\mu}$ energy spectrum. 63

5.6 The weighting for the $\bar{\nu}_{\mu}$ cross-section uncertainty. . . . . . . . . . . . 64

5.7 Data used in the fit compared to all the data. . . . . . . . . . . . 65

5.8 The $\chi^{2}$ surface for different smearing widths. . . . . . . . . . . . 68

5.9 Fit results for the neutrino energy of the $\nu_{\mu}$ sample. . . . . . . . . . 70

5.10 Fit results for the neutrino energy of the $\nu_{\mu}$ sample. . . . . . . . . 71

5.11 Fit results for the neutrino energy of the $\bar{\nu}_{\mu}$ sample. . . . . . . . . . . 72

5.12 Fit results for the neutrino energy of the $\bar{\nu}_{\mu}$ sample. . . . . . . . . . . 73

5.13 Fit results for the muon energy of the $\nu_{\mu}$ sample. . . . . . . . . . . . 74

5.14 Fit results for the muon energy of the $\nu_{\mu}$ sample. . . . . . . . . . . 75

5.15 The results on hadron production from the fit. . . . . . . . . . . 79

5.16 The $\pi^{+} / \pi^{-}$from the fit. . . . . . . . . . . . . . . . 80

5.17 Particle production results from the fit. . . . . . . . . . . . 81

5.18 Particle production from the fit compared to MIPP. . . . . . . . . . 82

5.19 Systematic uncertainty on the $\nu_{\mu} \mathrm{F} / \mathrm{N}$ flux ratio from hadron production. $\quad 85$

5.20 Systematic uncertainty on the $\bar{\nu}_{\mu} \mathrm{F} / \mathrm{N}$ flux ratio from hadron production. $\quad 86$

5.21 Total Systematic uncertainty on the $\nu_{\mu} \mathrm{F} / \mathrm{N}$ flux ratio. . . . . . . . . 87

5.22 The correlation matrix from the fit. . . . . . . . . . . . . . 88

5.23 A comparison of the systematic uncertainty before and after the fit. . . 90

6.1 Energy plots from LE RunI. . . . . . . . . . . . . . . . . . . . . . . . 94

6.2 Vertex and track end plots from LE RunI. . . . . . . . . . . . . . . . 95

6.3 Miscellaneous plots from LE RunI. . . . . . . . . . . . . . . . . . . 96

6.4 CC selection variables from LE RunI. . . . . . . . . . . . . . . . 97

6.5 CC selector plots for different energies from LE RunI. . . . . . . . . . . 98

6.6 CC selector plots for different energies on a log scale from LE RunI. . . 99

6.7 Energy plots from LE RunII. . . . . . . . . . . . . . . . . . . . . . . 101

6.8 Vertex and track end plots from LE RunII. . . . . . . . . . . . . . . . . 102

6.9 Miscellaneous plots from LE RunII. . . . . . . . . . . . . . . . . . . . . 103

6.10 CC selection variables from LE RunII. . . . . . . . . . . . . . . . . . . 104

6.11 CC selector plots for different energies from LE RunII. . . . . . . . . . . 105 
6.12 CC selector plots for different energies on a log scale from LE RunII. . . 106

6.13 Energy plots from HE RunII. . . . . . . . . . . . . . . . . . . . 108

6.14 Vertex and track end plots from HE RunII. . . . . . . . . . . . . . . . . 109

6.15 Miscellaneous plots from HE RunII. . . . . . . . . . . . . . . . . . . . . 110

6.16 CC selection variables from HE RunII. . . . . . . . . . . . . . . . . . . . 111

6.17 CC selector plots for different energies from HE RunII. . . . . . . . . . . 112

6.18 CC selector plots for different energies on a log scale from HE RunII. . . 113

6.19 The Far/Near ratio for different ND locations. . . . . . . . . . . . . 115

6.20 A matrix relating energies in the FD to the ND. . . . . . . . . . 116

6.21 Reweighting from the ND fit for different components. . . . . . . . . . 117

7.1 Selection variables for the kNN method. . . . . . . . . . . . . . 120

7.2 Selection variables for the PDF method. . . . . . . . . . . . . . 123

7.3 Selection variables for the PDF method. . . . . . . . . . . . . . . . . . 124

7.4 Selection variables for the ANN method. . . . . . . . . . . . . . 126

7.5 Selection variables for the ANN method. . . . . . . . . . . . . . . . 127

7.6 Sensitivity comparison of $\sin ^{2}\left(2 \theta_{32}\right)$ for different CC selectors . . . . . 129

7.7 Sensitivity comparison of $\Delta m_{32}^{2}$ for different CC selectors . . . . . . . 130

7.8 Efficiency and purity of the kNN selector. . . . . . . . . . . . . . . 131

7.9 Sensitivity comparison of different CC selectors. . . . . . . . . . . . 131

7.10 Sensitive comparison when the minimum hits for a track is changed. . . 133

8.1 The coordinate system used to measure the geomagnetic field. . . . . . . 135

8.2 Measurements of the geomagnetic field in the decay pipe. . . . . . . . 136

8.3 The impact of the geomagnetic field at the ND . . . . . . . . . . . 137

8.4 The impact of the geomagnetic field at the FD . . . . . . . . . . . 137

8.5 The impact of the geomagnetic field on the $\mathrm{F} / \mathrm{N}$ ratio. . . . . . . . . 138

8.6 The number of neutrino events as a function of month. . . . . . . . . . . 139

8.7 The impact of a decrease in target density on the neutrino energy spectrum. 140

8.8 The impact of removing target segments on the neutrino energy spectrum. 141

8.9 The change in the $\nu_{\mu}$ energy spectrum compared to the uncertainty. . . 142

8.10 The efficiencies as calculated from the hand scan of events. . . . . . . . 143

8.11 The uncertainty on the hadronic energy scale. . . . . . . . . . . . . . . 145

8.12 The ratio of selected muon removed data events to Monte Carlo. . . . . . 146

8.13 The uncertainty on the $\mathrm{F} / \mathrm{N}$ ratio from the flux. . . . . . . . . . . 148

8.14 The correlation matrices between different energy bins. . . . . . . . . . . 150

8.15 The error from the extrapolation procedure for the LE beam. . . . . . . 151

8.16 The difference between the data and MC from the ND fit. . . . . . . . 151 
8.17 Shifts in oscillation parameters from global systematic errors.

8.18 Shifts in oscillation parameters from global systematic errors using the ND reweighting. . . . . . . . . . . . . . . . . . . . 157

8.19 Shifts in oscillation parameters from relative systematic errors. . . . . . 159

9.1 The time difference between neutrino events and the closest spill. . . . . 163

9.2 The number of total neutrino events as a function of time. . . . . . . . . 164

9.3 FD energy plots for the LE beam. . . . . . . . . . . . . . . . . 167

9.4 FD CC selection variables for the LE beam. . . . . . . . . . . . . . . 168

9.5 FD vertex and track end plots for the LE beam. . . . . . . . . . . . . 169

9.6 2-D FD vertex and track end plots for the LE beam. . . . . . . . . . . 170

9.7 FD energy plots for the HE beam. . . . . . . . . . . . . . . . . 171

9.8 FD CC selection variables for the HE beam. . . . . . . . . . . . . . . . 172

9.9 FD vertex and track end plots for the HE beam. . . . . . . . . . . . . 173

9.10 2-D FD vertex and track end plots for the LE beam. . . . . . . . . . . . 175

9.11 FD neutrino energy spectrum for the LE RunI beam. . . . . . . . . . . 176

9.12 FD neutrino energy spectrum for the LE RunII beam. . . . . . . . . . . 177

9.13 FD neutrino energy spectrum for the HE beam. . . . . . . . . . . . . . 178

9.14 Confidence limits . . . . . . . . . . . . . . . . . . . . . . . . . . 179

9.15 Confidence limits with $\sin ^{2}\left(2 \theta_{32}\right)$ unconstrained . . . . . . . . . . . 180

9.16 Background components in the FD . . . . . . . . . . . . . . 181

9.17 Confidence limits for individual runs . . . . . . . . . . . . . . . . . 182

9.18 Results excluding cuts on event selection. . . . . . . . . . . . . . 183

9.19 A comparison of the $90 \%$ C.L. when the largest systematic errors are included as nuisance parameters (red) and with no systematics (black). . . 184

9.20 Comparison with the recent published MINOS results. . . . . . . . . . . 185

9.21 Combined LE RunI+RunII FD spectrum. . . . . . . . . . . . . . . . . 186

9.22 The effect of selection cuts on the $5 \mathrm{GeV}$ dip. . . . . . . . . . . . . 187

9.23 The neutrino energy spectrum in different regions of $y$. . . . . . . . . 188

9.24 Various quantities for events with energy from 3-7 GeV . . . . . . . . . . 189

9.25 The neutrino spectrum with additional focusing errors in the fit. . . . . 191

9.26 Oscillations compared to neutrino decay and decoherence. . . . . . . . . 194

9.27 The $\chi^{2}$ as a function of the energy exponent. . . . . . . . . . . . . 195

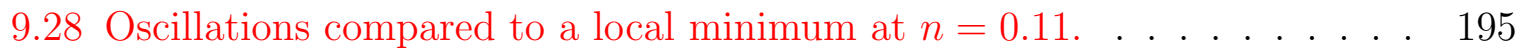

$9.2990 \%$ confidence limits for the data and Monte Carlo. . . . . . . . . . . . 196

9.30 The $\Delta \chi_{90}^{2}$ surface. . . . . . . . . . . . . . . . . . . . . . 198

$9.3190 \%$ confidence limits using the Feldman-Cousins prescription. . . . . . 199 
9.32 Distribution of oscillation parameters for different exposures. . . . . . . 201

9.33 Errors on the oscillation parameters as a function of exposure. . . . . . 202

9.34 Errors on the oscillation parameters as a function of $1-1 / \sqrt{\text { exposure. }}$. 203

9.35 Errors on the systematic parameters as a function of exposure. . . . . . 204

10.1 The confidence limits from this analysis compared to other experiments. 207

10.2 The sensitivity with increasing POT. . . . . . . . . . . . 208

A.1 The change in the $\nu_{e}$ flux to different uncertainties for the LE beam. . . 212

A.2 The change in the $\nu_{e}$ flux to different uncertainties for the HE beam. . . 213

A.3 The change in the $\nu_{e}$ flux less than $10 \mathrm{GeV}$ for the LE beam. . . . . . . 214

A.4 The change in the $\nu_{e}$ flux less than $10 \mathrm{GeV}$ for the HE beam. . . . . . . 215 


\section{List of Tables}

3.1 Different beam configurations in MINOS. . . . . . . . . . . . . . 31

4.1 The amount of data collected in different beam configurations. . . . . . 44

5.1 Amount of data and MC used for the $\nu_{\mu}$ sample in the fit. . . . . . . 66

5.2 Amount of data and MC used for the $\bar{\nu}_{\mu}$ sample in the fit. . . . . . . . 67

$5.3 \chi 2^{2}$ contributions from different beam configurations. . . . . . . . . 76

5.4 Hadron production parameters from the fit. . . . . . . . . . . 77

5.5 Target parameters from the fit. . . . . . . . . . . . . 78

5.6 Energy, NC background and cross-section parameters from the fit. . . . 78

8.1 Uncertainties from the Calibration Detector. . . . . . . . . . . . . . 144

8.2 Fit values obtained from fitting fake data in the ND. . . . . . . . . . . 153

8.3 Fit values obtained from fitting fake data in the ND with reweighting. . 153

8.4 Shifts in oscillation parameters from global systematic errors. . . . . . . 154

8.5 Shifts in oscillation parameters from global systematic errors. . . . . . . 156

8.6 Shifts in oscillation parameters from relative systematic errors. . . . . . 158

9.1 Amount of data used in this analysis. . . . . . . . . . . . . . 161

9.2 Fraction of events remaining after cuts. . . . . . . . . . . . . . . 165

9.3 The $\chi^{2}$ contribution from different beams. . . . . . . . . . . . . . . 181

9.4 Oscillation parameters from individual runs. . . . . . . . . . . . . . 182

9.5 Comparison with the different published MINOS results. . . . . . . . . 185

A.1 Systematic errors from focusing for $\nu_{e}$ events for the LE beam. . . . . . 216

A.2 Systematic errors from focusing for $\nu_{e}$ events for the HE beam. . . . . . 216 


\section{Chapter 1}

\section{Introduction}

During the mid-1990s the question of whether neutrinos oscillated had not been resolved. Atmospheric and solar neutrino experiments hinted at oscillations with large mixing angles, but other experiments had reported no evidence. Mixing from the quark sector and the hope for the neutrino as a dark matter candidate pointed to small values for the mixing angles. In the midst of these issues, the Main Injector Neutrino Oscillation Search (MINOS) was proposed to use an accelerator based neutrino beam to confirm oscillations and precisely determine the neutrino mixing parameters.

MINOS utilizes the NuMI (Neutrinos from the Main Injector) beam and two nearly identical detectors to study oscillations. The beam extracts $120 \mathrm{GeV}$ protons from the Main Injector at Fermi National Laboratory (Fermilab). The protons strike a graphite target creating showers of hadronic particles that decay to neutrinos. Muon neutrinos constitute over $99 \%$ of the NuMI beam with a peak energy of $\sim 3 \mathrm{GeV}$. The resulting combination of distance and energy gives MINOS good sensitivity to the neutrino mixing parameters suggested by atmospheric measurements. Under the approximation of twoflavor oscillations, the probability that a muon neutrino of energy $E$ will be detected as muon neutrino after having traveled a distance $L$ is:

$$
\operatorname{Prob}_{\text {survival }}=1-\sin ^{2}(2 \theta) \sin ^{2}\left(1.27 \frac{\Delta m^{2}\left[\mathrm{eV}^{2}\right] L[\mathrm{~km}]}{E[\mathrm{GeV}]}\right)
$$


where $\Delta m^{2}$ is the mass-squared difference between the two states and $\sin ^{2}(2 \theta)$ is the mixing angle. Two detectors observe and measure individual neutrino interactions for MINOS. The Near Detector is located at Fermilab and the Far Detector at the Soudan Underground Laboratory.

While the primary goal of MINOS is to determine the neutrino mixing parameters, it has a rich physics reach. MINOS can measure the sub-dominant oscillation mode from $\nu_{\mu}$ to $\nu_{e}$, dependent on the remaining unknown mixing parameters $\theta_{13}$ and $\delta$. Matter effects and the sign of $\Delta m_{32}^{2}$ also play a significant role in the $\nu_{\mu} \rightarrow \nu_{e}$ rate. MINOS can also make measurements of neutrino cross-sections, the form factors in quasi-elastic scattering processes, properties of high energy cosmic rays, and atmospheric neutrinos.

\subsection{Measuring the Atmospheric Oscillation Parame- ters}

Protons from the Main Injector (MI) create pions and kaons producing neutrinos via the following decays:

$$
\begin{gathered}
\pi^{+} \rightarrow \nu_{\mu}+\mu^{+}(99.9 \%), \\
\pi^{-} \rightarrow \bar{\nu}_{\mu}+\mu^{-}(99.9 \%), \\
K^{+} \rightarrow \nu_{\mu}+\mu^{+}(63.4 \%), \\
K^{-} \rightarrow \bar{\nu}_{\mu}+\mu^{-}(63.4 \%),
\end{gathered}
$$

where the branching fractions have been indicated in parenthesis. A majority of muons stop before they can decay to neutrinos, although they do provide the low rate of $\nu_{e}$ events in the beam. To increase the number of neutrinos, the pions and kaons are "focused" toward the detectors by toroidal magnets that preferentially select positively charged pions and kaons. 
Oscillations are seen as an energy dependent deficit of muon type neutrinos at the Far Detector relative to the Near. The two detector arrangement minimizes the systematic uncertainty due to the neutrino flux, cross-sections, and detector efficiencies. Differences between Near and Far must be understood to avoid being mistaken for oscillations. Unfortunately, there are some differences between the two, the largest being the amount of solid angle seen by each detector. Using only the $1 / r^{2}$ difference, the Far should see $\left(r_{\text {Near }} / r_{F a r}\right)^{2} \approx 2 \times 10^{-6}$ fewer events. Other differences come from the fact that the Near sees an extended neutrino source while the Far a point source. An extrapolation procedure is necessary, using the Near Detector data as a reference point, to predict the expected neutrino flux at the Far Detector.

This thesis describes the process of measuring the atmospheric oscillation parameters with MINOS and is organized in the following manner. Chapter 2 recounts the historical context of neutrino oscillations and how our understanding has evolved as a result of theoretical advances and experimental discoveries. The standard theory underlying neutrino oscillations will also be reviewed in the two neutrino framework. Chapter 3 discusses the details of the NuMI beam and the MINOS detectors in more detail. In Chapter 4, various reconstruction and simulation codes are described.

Due to our lack of knowledge of the neutrino flux, a prediction of the expected number of neutrino events in the Near Detector cannot be calculated from first principles. A method using the data in the Near Detector to constrain and tune the Monte Carlo calculation is introduced in Chapter 5. These constraints reduce the systematic uncertainties from the neutrino beam. The full implications of this tuning are explored in Chapter 6, where different physics distributions between the Near Detector data and the tuned Monte Carlo are compared. This tuning serves as an extrapolation procedure for predicting the neutrinos at Far Detector in the absence of oscillations.

Chapter 7 looks at the discrimination of Charged Current from Neutral Current events. This selection is important because Charged Current events allow for the determination of the neutrino flavor. Different discrimination methods are evaluated based 
on their sensitivity to the oscillation parameters. Chapter 8 describes the impact of systematic errors on the measurement. The Far Detector data set, obtained from exposure to $3.36 \times 10^{20}$ protons on target, is examined in Chapter 9. The data is found to be in good agreement with the oscillation hypothesis. Finally, Chapter 10 summarizes the conclusions and compares this measurement to previous experiments.

In the course of this thesis, reference is made to work carried out by other members of the MINOS collaboration; where appropriate the attribution of figures and results to specific sources is given. Unless indicated as such, the results, tables and figures are original to this thesis. 


\section{Chapter 2}

\section{Background}

The neutrino was proposed in 1930 [1] as a 'desperate remedy' to save the principle of the conservation of energy which had been seemingly violated in observations of nuclear decay. At that time, only two elementary particles were known - the electron and proton. Furthermore, the new particle was considered undetectable due it being neutral and weakly interacting. As our understanding of particle physics unfolded, it became apparent that perhaps this neutrino could be detected. In 1953, experimenters saw the first evidence of the neutrino. After its detection the neutrino played a key role in providing a complete picture of the unification of the weak and electromagnetic forces. This chapter will review a brief history of the important historical events in the life of the neutrino. The theory of neutrino oscillations will be developed and the experimental evidence for oscillations will be reviewed.

\subsection{The Early Years of Neutrinos}

Studies in the early 20th century revealed that electrons, produced in $\beta$-decay of nuclei, had a continuous energy spectrum $[2,3,4,5]$. This posed a serious theoretical problem - if the electron was the result of a two-body decay, then assuming the conservation of energy and momentum, it should be mono-energetic. This lead Neils Bohr to believe 
that the conservation of energy only held statistically in this reaction. A revolutionary view, offered by Wolfgang Pauli [1] in 1930, held that energy was conserved, but that a very penetrating neutral particle, called the "neutron", was emitted along with the electron. This neutron, he postulated, could have a penetrating power 10 times larger than a photon (we now know it is closer to $10^{16}$ ).

In 1932, Chadwick [6] found evidence for a neutral particle in the nucleus. These particles were slightly heavier than the proton and named neutrons. Unfortunately, the neutron was too heavy to help explain the problem of $\beta$-decay. To distinguish the particle from the heavier neutron, Enrico Fermi named the particle emitted in $\beta$ decay the "neutrino" Fermi developed a theory of $\beta$ decay $[7,8]$ following methods similar to those of quantum electrodynamics. He postulated that the neutrino was created in a point interaction between an electron and a nucleon.

Twenty years later, Reines and Cowen, overcame many technical challenges to observe the first evidence for the neutrino [9]. They used a liquid scintillator detector doped with Cadmium and placed it outside of the Hanford nuclear reactor. They looked for the reaction $\bar{\nu}_{\mu}+p \rightarrow n+e^{+}$, where the positron would produce gamma rays in the scintillator followed by a prompt capture of the neutron by the Cadmium. They observed an average of $2.55 \pm 0.15$ counts/min when the reactor was on and $2.14 \pm 0.13$ counts/min with the reactor off. The difference of $0.41 \pm 0.2$ counts $/ \mathrm{min}$, was close to the predicted neutrino flux of $\sim \frac{1}{5}$ counts $/ \mathrm{min}$. Reines and Cowen improved and confirmed their measurement in 1956 [10] at the Savannah River Plant.

In 1962, researchers at Brookhaven [11] observed a second type of neutrino distinct from those which had been observed with electrons. This neutrino was seen in the decay of pions to muons and was dubbed the muon neutrino. With the discovery of the heavier tau lepton in 1975 [12], some postulated an associated third type of neutrino. The number of active neutrinos was also indirectly observed from measurements of the the $Z$ boson to be $2.994 \pm 0.012$ [13]. Direct evidence of the tau neutrino came in 2001 from the DONUT experiment [14]. 
The theoretical underpinnings of the weak interactions (those which govern neutrinos) were unified with the electromagnetic interactions by Glashow [15], Salam [16] and Weinberg [17]. They postulated that the electromagnetic and weak interactions were mediated by two massive gauge bosons, the $W$, and $Z$. The $W$ bosons were responsible for the neutrino interactions heretofore observed where a lepton is produced. These interactions were labeled Charged Current (CC). The theory also predicted Neutral Current (NC) interactions via the neutral $Z$ boson. The Gargamelle experiment observed the first neutral current neutrino interaction in 1973 [18].

\subsection{Neutrino Mixing}

Drawing upon evidence of mixing in the kaon system [19, 20], Pontecorvo applied the analogy to neutrinos [21]. Only one type of neutrino was known at the time, so he only considered $\nu \rightarrow \bar{\nu}$ transitions. After the discovery of the muon neutrino, he expanded his work with a paper [22] considering the non-conservation of leptonic charge and possible transitions of $\nu_{\mu} \leftrightarrow \nu_{e}$. Several years earlier Maki, Nakagawa, and Sakata [23] had introduced a formalism for two neutrino oscillations (although their work was relatively unknown).

The formalism assumes that neutrinos are created in flavor or weak eigenstates. The flavor is defined by the accompanying lepton created with the neutrino. Although neutrinos interact in flavor eigenstates, they propagate as mass eigenstates. If neutrinos are massive, then the mass states could differ from the flavor eigenstates. A neutrino of flavor $\alpha$ can then be described as a linear combination of mass eigenstates:

$$
\left|\nu_{\alpha}>=\sum_{i} U_{\alpha i}^{\star}\right| \nu_{i}>
$$

where the sum $i$ is over all the mass eigenstates and $U^{\star}$ is the leptonic mixing matrix. The mixing matrix is a $3 \times 3$ unitary matrix, sometimes referred to as the PMNS matrix 
(named after Pontecorvo, Maki, Nakagawa, Sakata) that describes the transformation between the weak and mass eigenstates.

In the neutrino rest frame, the time evolution of the neutrino state, given by the Schrodinger equation, is

$$
\left|\nu_{i}\left(\tau_{i}\right)>=e^{-i m_{i} \tau_{i}}\right| \nu_{i}(0)>
$$

where $\tau_{I}$ is the proper time. By Lorentz invariance $m_{i} \tau_{i}=E_{i} t-p_{i} L$, where $L$ is the distance traveled in the lab frame and $t$ the time. For highly relativistic particles such as neutrinos $L \approx t$. For each mass eigenstate the momentum $p_{i}$ can be written as:

$$
p_{i}=\sqrt{E^{2}-m_{i}^{2}} \approx E-\frac{m_{i}^{2}}{2 E}
$$

Assuming each eigenstate has the same energy gives

$$
m_{i} \tau_{i}=E-\left(E-\frac{m_{i}^{2}}{2 E}\right) L=\frac{m_{i}^{2} L}{2 E}
$$

The evolution of the neutrino state after having traveled a distance $L$ can now be expressed as

$$
\left|\nu_{\alpha}(L)>=\sum_{i} U_{\alpha i}^{\star} e^{-i \frac{m_{i}^{2} L}{2 E}}\right| \nu_{i}>
$$

The amplitude that a neutrino of flavor $\alpha$ will be detected as a flavor $\beta$ is given by

$$
\begin{aligned}
\operatorname{Amplitude}\left(\nu_{\alpha}->\nu_{\beta}\right) & =<\nu_{\beta} \mid \nu_{\alpha}(L)> \\
& =<\nu_{\beta}\left|\sum_{\beta}\left(\sum_{i} U_{\alpha i}^{\star} e^{-i \frac{m_{i}^{2} L}{2 E}}\right) U_{\beta i}\right| \nu_{\beta}> \\
& =\sum_{i} U_{\alpha i}^{\star}\left(e^{-i \frac{m_{i}^{2} L}{2 E}}\right) U_{\beta i} .
\end{aligned}
$$


The probability for detecting the neutrino as a flavor $\beta$ is

$$
\begin{aligned}
\operatorname{Prob}\left(\nu_{\alpha}->\nu_{\beta}\right) & =\left|\operatorname{Amplitude}\left(\nu_{\alpha}->\nu_{\beta}\right)\right|^{2} \\
& \left.=\sum_{i j} U_{\beta i} U_{\alpha i}^{\star} U_{\beta j}^{\star} U_{\alpha j} e^{-i \frac{\left(m_{i}^{2}-m_{j}^{2}\right) L}{2 E}}\right),
\end{aligned}
$$

which can be written as

$$
\begin{aligned}
\operatorname{Prob}\left(\nu_{\alpha}->\nu_{\beta}\right)= & \sum_{i} U_{\beta i} U_{\alpha i}^{\star} \sum_{j} U_{\beta j}^{\star} U_{\alpha j} \\
& +\sum_{i j} U_{\beta i} U_{\alpha i}^{\star} U_{\beta j}^{\star} U_{\alpha j}\left(e^{-i \frac{\Delta m_{i j}^{2} L}{2 E}}-1\right),
\end{aligned}
$$

where $\Delta m_{i j}^{2}=\left(m_{i}^{2}-m_{j}^{2}\right)$. Because of the unitarity of $U$, The first term is $\delta_{i j}$. The second term can be simplified by noting that when $i=j$ the term vanishes and that the terms for $j>i$ are complex conjugates of $j<i$. Hence,

$$
\operatorname{Prob}\left(\nu_{\alpha}->\nu_{\beta}\right)=\delta_{\alpha \beta}+2 \sum_{i>j} \mathfrak{R e}\left[U_{\beta i} U_{\alpha i}^{\star} U_{\beta j}^{\star} U_{\alpha j}\right]\left(e^{-i \frac{\Delta m_{i j}^{2} L}{2 E}}-1\right)
$$

Splitting the final part into real and imaginary parts gives:

$$
\begin{aligned}
\operatorname{Prob}\left(\nu_{\alpha}->\nu_{\beta}\right)= & \delta_{\alpha \beta} \\
& -4 \sum_{i>j} \mathfrak{R e}\left[U_{\beta i} U_{\alpha i}^{\star} U_{\beta j}^{\star} U_{\alpha j}\right] \sin ^{2}\left(\frac{\Delta m_{i j}^{2} L}{4 E}\right) \\
& +2 \sum_{i>j} \mathfrak{I m}\left[U_{\beta i} U_{\alpha i}^{\star} U_{\beta j}^{\star} U_{\alpha j}\right] \sin \left(\frac{\Delta m_{i j}^{2} L}{2 E}\right)
\end{aligned}
$$

The same answer can be derived under the assumption that the mass eigenstates have equal momentum as opposed to equal energy. 
The expression can be simplified for the case of two neutrino states

$$
\operatorname{Prob}\left(\nu_{\alpha}->\nu_{\alpha}\right)=1-4 \sum_{i>j} \sum_{j}\left|U_{\alpha i}\right|^{2}\left|U_{\alpha j}\right|^{2} \sin ^{2}\left(\frac{\Delta m_{i j}^{2} L}{4 E}\right) .
$$

For two neutrino states the mixing matrix becomes:

$$
U=\left(\begin{array}{cc}
\cos \theta & \sin \theta \\
-\sin \theta & \cos \theta
\end{array}\right)
$$

simplifying the survival probability to

$$
\begin{aligned}
& \operatorname{Prob}\left(\nu_{\alpha}->\nu_{\alpha}\right)=1-\sin ^{2}(2 \theta) \sin \left(\frac{\Delta m^{2} L}{4 E}\right) \\
& \operatorname{Prob}\left(\nu_{\alpha}->\nu_{\beta}\right)=\sin ^{2}(2 \theta) \sin \left(\frac{\Delta m^{2} L}{4 E}\right) .
\end{aligned}
$$

Putting the expression in SI units gives:

$$
\operatorname{Prob}\left(\nu_{\alpha}->\nu_{\alpha}\right)=1-\sin ^{2}(2 \theta) \sin \left(\frac{1.27 \Delta m^{2} L[k m]}{2 E[G e V]}\right)
$$

Two flavor oscillations are dependent on a mixing angle $\theta$, the mass-squared difference $\Delta m^{2}, L$, the distance traveled and $E$ the energy of the neutrino. Experiments define the combination of $L / E$ which sets the sensitivity to a particular oscillation length.

When extending the formalism to three neutrinos four free parameters are allowed for a unitary matrix. They are usually defined as 3 mixing angles $\theta_{12}, \theta_{23}, \theta_{13}$ and one CP 

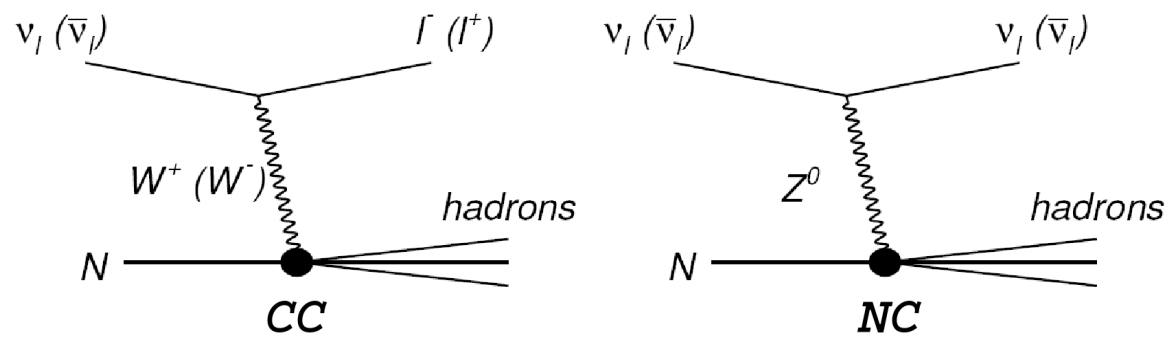

Figure 2.1: Left: A charged current (CC) interaction, where a neutrino of type $l$ interacts with a nucleon via the exchange of a $W$ boson and creates a lepton of type $l$. Right: A neutral current (NC) interaction where the neutrino scatters off a nucleon via the exchange of a $Z$ boson.

violating phase $\delta$. The mixing matrix $U$ is commonly factorized by:

$$
\begin{aligned}
& U=\left(\begin{array}{ccc}
U_{e 1} & U_{e 2} & U_{e 3} \\
U_{\mu 1} & U_{\mu 2} & U_{\mu 3} \\
U_{\tau 1} & U_{\tau 2} & U_{\tau 3}
\end{array}\right) \\
& =\left(\begin{array}{ccc}
1 & 0 & 0 \\
0 & c_{23} & s_{23} \\
0 & -s_{23} & c_{23}
\end{array}\right)\left(\begin{array}{ccc}
c_{13} & 0 & s_{13} e^{-i \delta} \\
0 & 1 & 0 \\
-s_{13} e^{i \delta} & 0 & c_{13}
\end{array}\right)\left(\begin{array}{ccc}
c_{12} & s_{12} & 0 \\
-s_{12} & c_{12} & 0 \\
0 & 0 &
\end{array}\right) \text {, } \\
& =\left(\begin{array}{ccc}
c_{12} c_{13} & s_{12} c_{13} & s_{13} e^{-i \delta} \\
-s_{12} c_{23}-c_{12} s_{23} s_{13} e^{i \delta} & c_{12} c_{23}-s_{12} s_{23} s_{13} e^{i \delta} & s_{23} c_{23} \\
s_{12} s_{23}-c_{12} c_{23} s_{13} e^{i \delta} & -c_{12} s_{23}-s_{12} c_{23} s_{13} e^{i \delta} & c_{23} c_{13},
\end{array}\right)
\end{aligned}
$$

where $s_{i j}=\sin \theta_{i j}$, and $c_{i j}=\cos \theta_{i j}$. When including three neutrinos, the survival probability is more complicated and will not be given here.

\subsection{Neutrino Interactions}

The theory of weak interactions predicts two types of neutrino interactions: Charged Current (CC), and Neutral Current (NC) (see Figure 2.1). CC interactions are described 
by the following reaction

$$
\nu_{l}+N \rightarrow l+\text { hadrons }
$$

and NC by

$$
\nu_{l}+N \rightarrow \nu_{l}+\text { hadrons }
$$

where $N$ is a nucleon and $l$ is a lepton. The key difference between the two is that CC interactions produce a detectable lepton in the final state. Identification of the lepton allows for the determination of the flavor of neutrino.

$\mathrm{CC}$ reactions can be decomposed into three distinct sectors: quasi-elastic, resonance and deep inelastic scattering. They are characterized (see Figure 2.2) by:

$$
\begin{aligned}
& \nu_{l}+N \rightarrow l+N \quad \text { Quasi - Elastic (QE), } \\
& \nu_{l}+N \rightarrow l+\Delta / N^{\star}->l+\pi+N^{*} \quad \text { Resonance (RES), } \\
& \nu_{l}+N \rightarrow l+X \quad \text { Deep Inelastic Scattering (DIS), }
\end{aligned}
$$

where $N^{*}$ and $\Delta$ are excited states of the nucleus. For low energy $(<1 \mathrm{GeV})$ neutrinos, a majority of interactions are QE. QE events are the simplest events with a lepton and nucleon in the final state. At intermediate energies, RES interactions produce excited states of the nucleon decaying with $\pi$ production. Theoretically this is the most complicated channel to describe because the nucleon must be represented by a bound 3 quark system. Nuclear effects and possible re-interactions of particles in the nucleus make it especially challenging. At high energies DIS interactions dominate where the neutrinos interacts with individual partons of the nucleons.

The dynamics can be described by a V-A Current-Current Lagrangian. The vector portion of the weak interaction is related to the electromagnetic current via the "CVC theorem" or conserved vector current theorem. This allows the vector portion of the weak interaction to be determined from electron scattering experiments leaving the axial component as the most uncertain component. The form factor characterizing the axial 

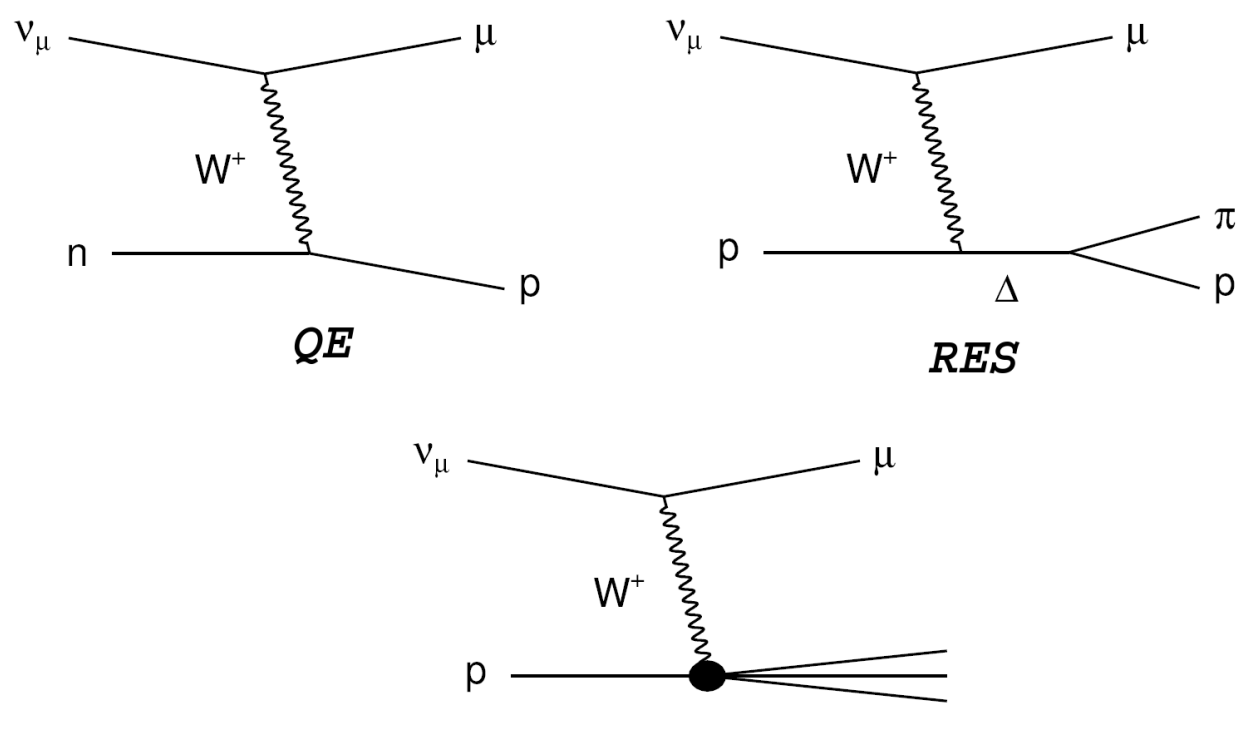

DIS

Figure 2.2: Three types of CC interactions: quasi-elastic or QE (top left), resonance or RES (top right), and deep inelastic scattering or DIS (bottom).

part of the hadronic current is usually assumed to have a dipole form, leaving a single unknown parameter - the axial vector mass $M_{A}$.

\subsection{Solar Neutrino Problem}

Through nuclear processes in the sun, large amounts of neutrinos are created. Ray Davis first attempted to detect these neutrinos in 1955 at Hanford [24], but was unsuccessful. His experiment used a tank of $\mathrm{C}_{2} \mathrm{CL}_{4}$ and looked for neutrinos using a radio-chemical method proposed by Pontecorvo [25] in 1946. The process proceeds via $\nu_{e}+\mathrm{Cl}^{37} \rightarrow \mathrm{Ar}^{37}+e^{-}$, where a neutrino interacts with the the chlorine and the argon atoms are individually extracted and counted. In 1968, after improving the detector and moving it the Homestake Mine, Davis published the detection of solar neutrinos [26]. He reported less than half the number of neutrinos expected from solar model calculations [27]. Both theory and experiment continued improving for the next twenty years, with results largely unchanged. One possibility put forth by Gribov [28], was that the 
deficit of neutrinos from the sun was due to neutrinos changing from one type to another in flight to the earth. The scientific community, however, did not trust the solar models sufficiently to consider the observed deficit a problem.

Confirming evidence for a deficit of solar neutrinos came in 1988 [29] from the Kamiokande experiment. They observed $0.46 \pm 15 \%$ less than the expected solar neutrino flux. Evidence continued to increase as data from other radio-chemical experiments Sage [30] and Gallex [31] confirmed these results.

Direct evidence for flavor change came from The Sudburry Neutrino Observatory (SNO). SNO was sensitive to NC interactions in addition to the $\mathrm{CC}$ seen by previous solar neutrino experiments. Since all neutrino flavors participate in the NC interaction, they should not show a deficit, which is is precisely what they observed [32] as shown in in Figure 2.3. The NC interactions were in agreement with solar models while the CC interactions were not.

Oscillations of electron antineutrinos from KamLAND [34], strengthened the evidence for oscillations. KamLAND measured antineutrinos from numerous nuclear reactors with an average distance of $180 \mathrm{~km}$ from the detector. Results from KamLAND and solar neutrinos are shown in Figure 2.4. It is a strong confirmation of the oscillation hypothesis that such different experiments agree. The oscillation parameters measured by these experiments were $\Delta m^{2}=7.9_{-0.5}^{+0.6} \times 10^{-5} \mathrm{eV}^{2}$ and $\tan ^{2} \theta=0.40_{-0.07}^{+0.10}$.

\subsection{Atmospheric Neutrino Problem}

High energy cosmic rays produce neutrinos via the decay of pions, kaons and muons. The interactions are:

$$
\begin{aligned}
p+N & \rightarrow n+\pi / K+\ldots \\
\pi^{ \pm} / K^{ \pm} & \rightarrow \mu^{ \pm}+\nu_{\mu} / \bar{\nu}_{\mu} \\
\mu^{ \pm} & \rightarrow e^{ \pm}+\bar{\nu}_{\mu} / \nu_{\mu}+\nu_{e} / \bar{\nu}_{e} .
\end{aligned}
$$




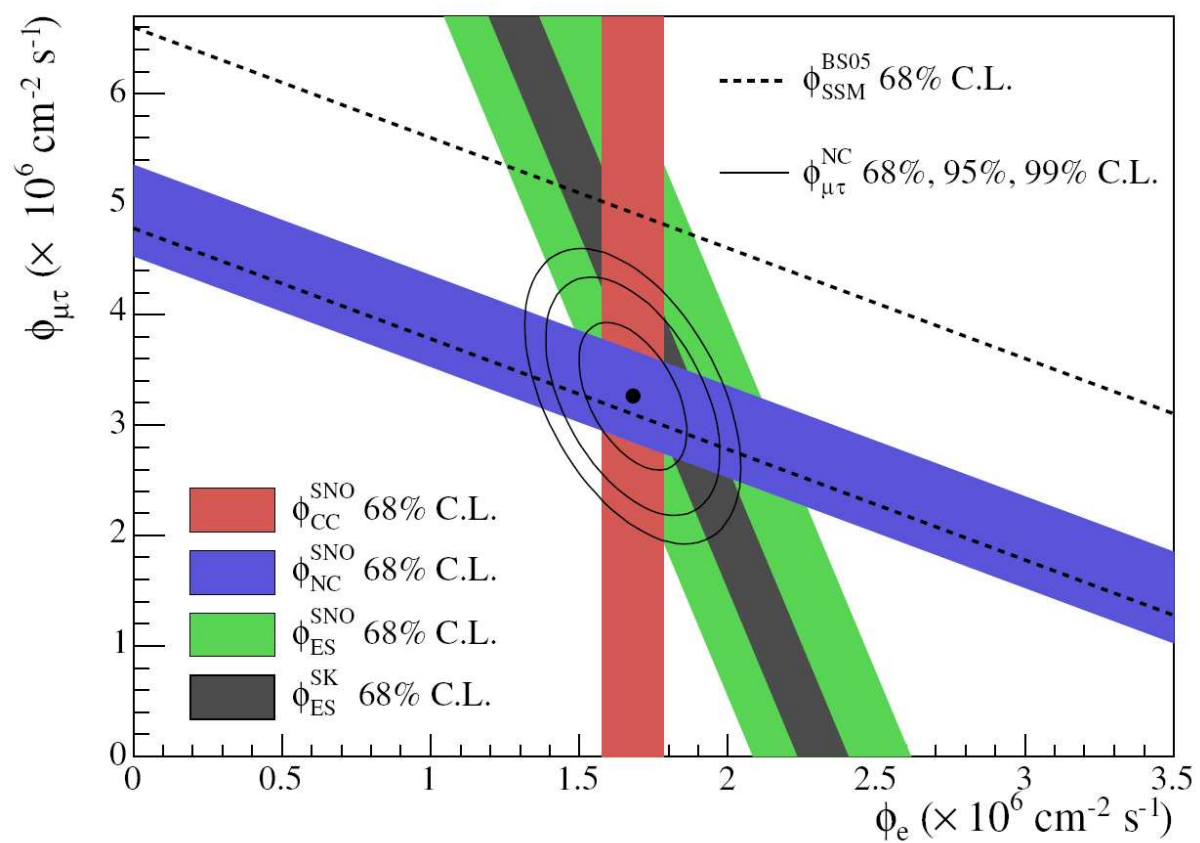

Figure 2.3: Flux of $\nu_{\mu}+\nu_{\tau}$ vs $\nu_{e}$ measured by SNO for charged current (red), neutral current (blue), elastic scattering (green), along with results from SuperKamiokande elastic scattering measurements (black). The solar standard model is shown by the dashed lines. Taken from [33]. 

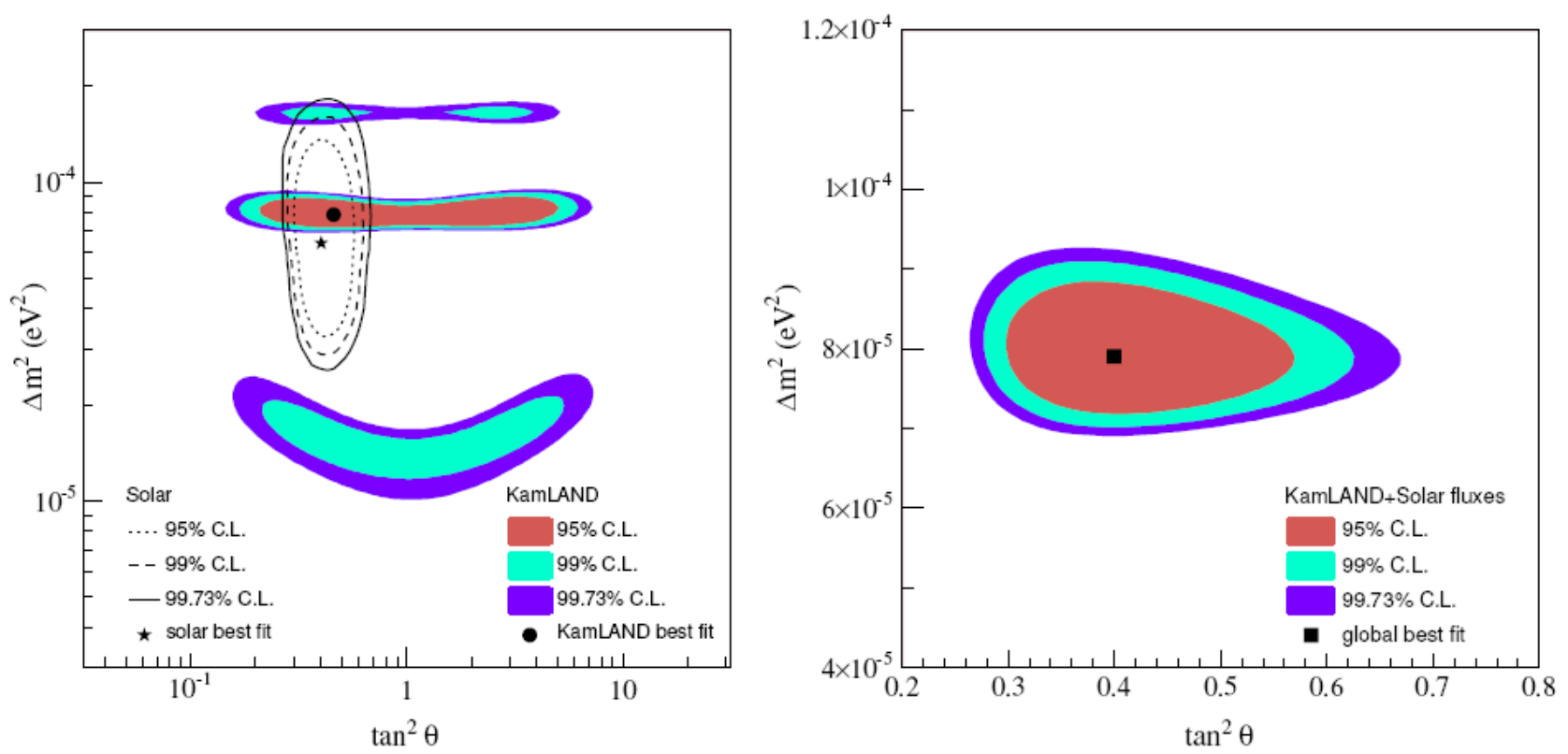

Figure 2.4: Left: Neutrino oscillations parameters allowed by KamLAND (shaded area) and solar neutrino experiments (lines). Right: A combined two neutrino analysis of solar and KamLAND data. Taken from [34].

Each initial hadron produces on average two muon neutrinos to each electron neutrino. Thus, the ratio of observed $\nu_{\mu}$ to $\nu_{e}$ should be $\approx 2: 1$. The ratio largely cancels out uncertainties on the atmospheric neutrino flux. Deep underground detectors, where backgrounds from cosmic rays are small, should be able to measure the flavor content via CC interactions.

Fortunately, proton decay experiments also require low cosmic ray backgrounds. In the late 1980s, two proton decay experiments observed an anomalous deficit in their expected backgrounds from atmospheric neutrinos. Both used Cerenkov radiation of muons and electrons to detect neutrinos. IMB [36] observed $26 \pm 3 \%$ of their events from muon decay when they expected $34 \pm 1 \%$. The Kamionkande [37] experiment observed an even larger discrepancy of $59 \pm 7 \%$ of the expected $\nu_{\mu}$ flux. The $\nu_{e}$ flux, in comparison, was seen to agree with expectations. The energies of these neutrinos were too large to 


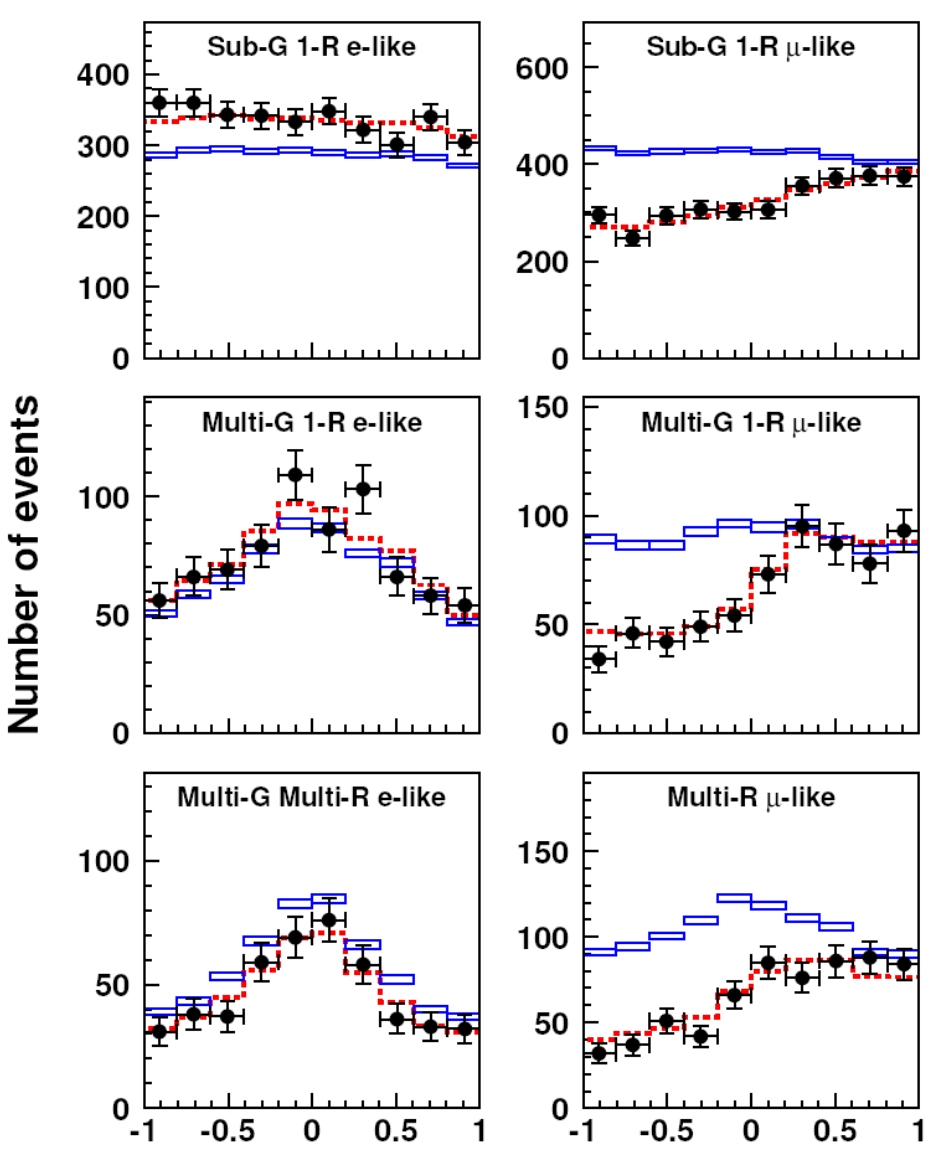

Figure 2.5: Zenith angle distributions for electron-like (left column) and muon-like (right column) events from the SuperKamiokande experiment. The black points are data, the blue boxes shows the expected values without oscillations and the red dashed line shows the best fit oscillation solution. Adapted from [35] 
be explained by the oscillation lengths consistent with solar neutrinos. This ruled out oscillations of $\nu_{\mu} \rightarrow \nu_{e}$. These required a separate oscillation length scale.

Complications arose when two iron calorimeter experiments, Frejus [38] and Nusex [39] observed no evidence for oscillations. Many considered the problem to lie with water Cerenkov experiments. A few years later, results from Soudan2 [40] strengthened the case for oscillations. These were iron calorimeters and therefore ruled out possible problems with water Cerenkov detectors. Convincing evidence finally came from the successor to Kamiokande, SuperKamiokande in 1998. SuperKamiokande [41] measured the ratio, $R=(\mu / e)_{D a t a} /(\mu / e)_{M C}$, of muon to electron neutrinos in data and Monte Carlo to be $R=0.63 \pm 0.03$ (stat.) \pm 0.05 (sys). They observed oscillations by looking at neutrinos that traveled different lengths (see Figure 2.5). Downward neutrino events arriving from above the detector travel a shorter distance than those arriving from below which travel through the earth. The dependence of the deficit on the zenith angle and the $R$ value strongly supported neutrino oscillations of $\nu_{\mu} \rightarrow \nu_{\tau}$. Recently, SuperKamiokande has published results [42] where events are binned as a function of $L / E$. The sinusoidal nature of the oscillations is clearly visible.

The K2K [43] experiment confirmed the results of the atmospheric oscillations using a controlled source of neutrinos. Using $12 \mathrm{GeV}$ protons, a beam of neutrinos was produced and sent $250 \mathrm{~km}$ to the SuperKamiokande detector. At this distance and energy, K2K is sensitive to the same region of parameter space as the other atmospheric neutrino experiments. A smaller $1 \mathrm{kT}$ water Cerenkov detector was located $300 \mathrm{~m}$ from the target. A total of 112 events were seen at SuperKamiokande where 158 were expected. The energy spectrum of these events is shown in Figure 2.7 


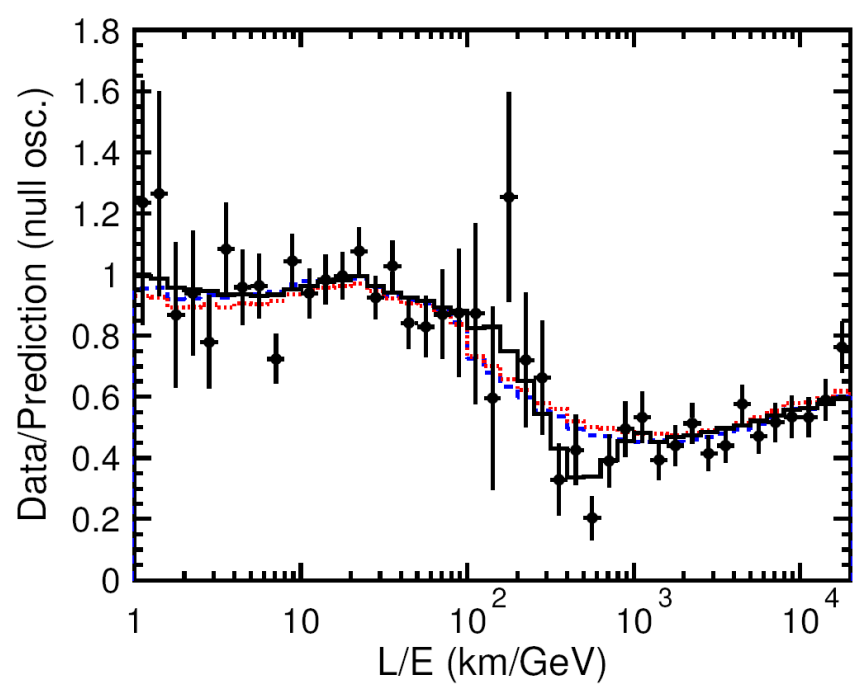

Figure 2.6: The ratio of data to Monte Carlo events with and without neutrino oscillations as a function of $L / E$ as measured by the SuperKamiokande experiment. The line indicates the best fit oscillation solution (black), neutrino decay (dashed blue line), neutrino decoherence (dotted red line). Taken from [42]

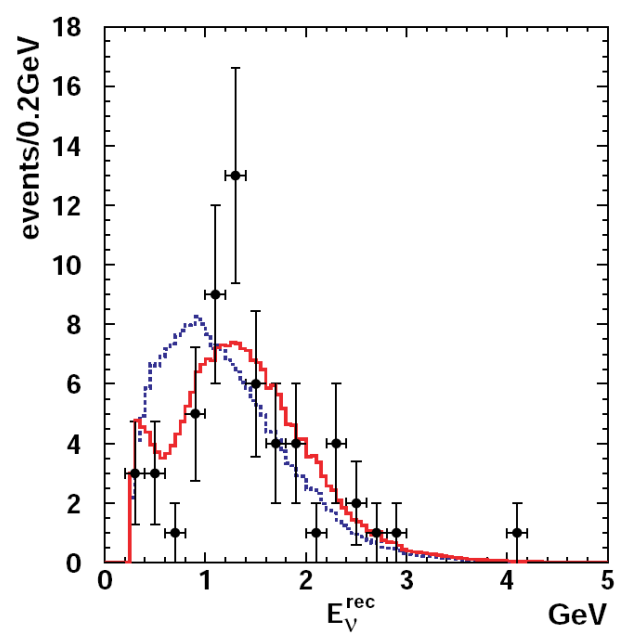

Figure 2.7: The ratio of data to Monte Carlo events with and without neutrino oscillations as a function $L / E$ as measured by the $\mathrm{K} 2 \mathrm{~K}$ experiment. The line indicates the best fit oscillation solution (red), the unoscillated prediction (blue) and the data (points). Taken from [43] 


\section{Chapter 3}

\section{The NuMI Beam and the MINOS Detectors}

\subsection{The NuMI Beam}

The Neutrinos at the Main Injector (NuMI) beamline delivers neutrinos to the MINOS detectors. A diagram of the beamline is illustrated in Figure 3.1. Protons are extracted from the Main Injector in a $10 \mu$ s spill and bent downward toward the target. Downstream of the target are two magnetic focusing horns that focus the secondary particles to the decay pipe. While in the decay pipe the secondary particles decay to neutrinos.

\subsubsection{Proton Beam}

The protons delivered to NuMI begin as $\mathrm{H}^{-}$ions and are accelerated in a Cockroft-Walton accelerator to $750 \mathrm{keV}$. A linear accelerator increases their energy from $750 \mathrm{keV}$ to 400 $\mathrm{MeV}$ for injection into the Booster. Before entering the Booster, a carbon foil converts the $\mathrm{H}^{-}$ions into protons. The Booster captures the protons and increases their energy to $8 \mathrm{GeV}$. The final stage of acceleration is provided by the Main Injector (MI) where they are accelerated to $120 \mathrm{GeV}$. The large radius of the MI allows for multiple batches of protons from the Booster to be accelerated simultaneously. Six proton batches fit inside 

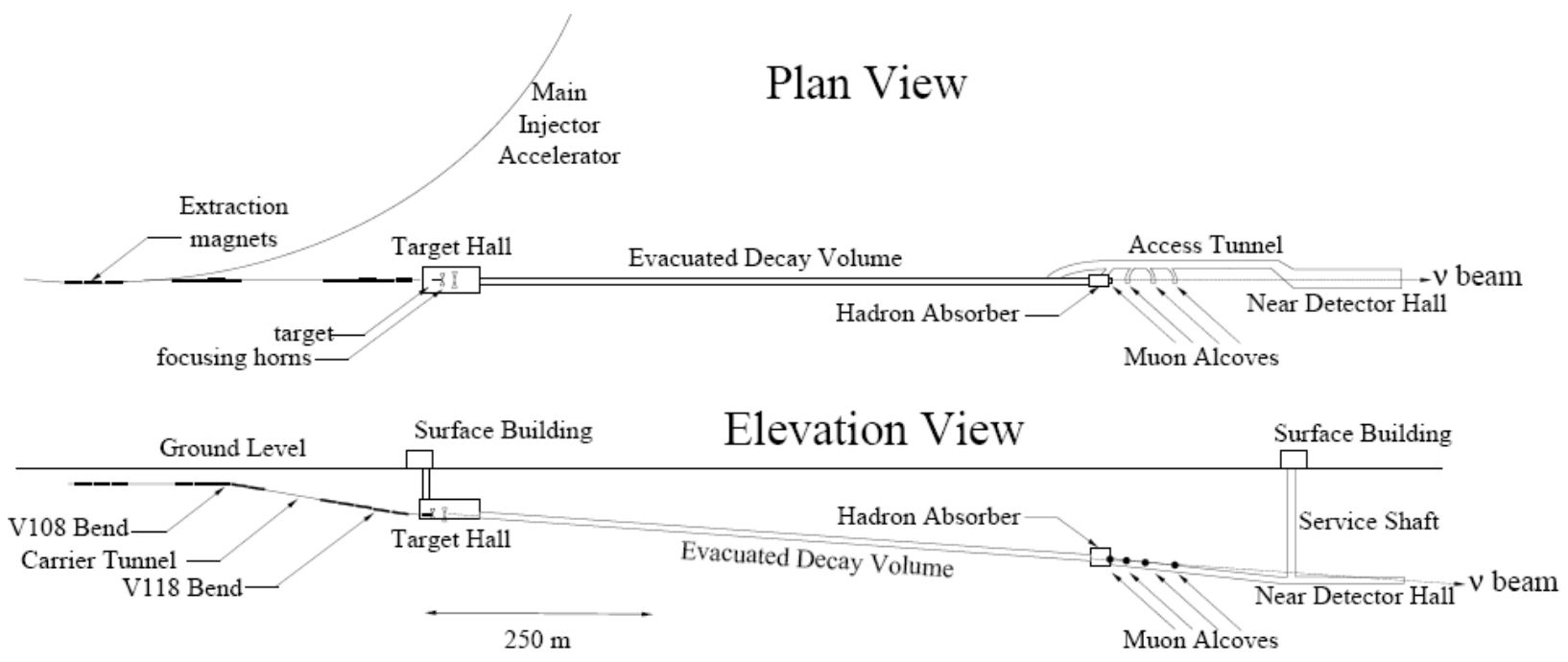

Figure 3.1: A plan and elevation view of the NuMI beam where protons from the MI are impinged upon the NuMI target and focused via magnetic horns toward the MINOS detectors. Taken from [44].

the MI. This batch structure is clearly visible in the MINOS neutrino data. When NuMI receives all of the batches the extraction occurs via a $10 \mu$ s spill. Occasionally, one of the batches is sent to the Antiproton Accumulator for the Tevatron, in which case the spill is $8.6 \mu \mathrm{s}$.

Each extraction contains $\sim 2.1 \times 10^{13}$ protons per bunch with a cycle time from 2.2 to 2.4 seconds [44]. After extraction, the protons travel $350 \mathrm{~m}$ at a downward angle of $58 \mathrm{mrad}$ to the NuMI target. Throughout the journey, the position and profile of the beam is monitored by Beam Position Monitors (BPMs) and Secondary Emission Monitors (SEMs). The beam intensity is measured by toroidal beam current transformers and through loss monitors. The position of the proton beam was stable to within $\pm 0.1 \mathrm{~mm}$.

\subsubsection{NuMI Target}

To reduce the stress induced by the deposited energy from the proton beam, the NuMI target is segmented. The target (see Figure 3.2) consists of 47 individual rectangular segments with an additional segment located further upstream used for beam alignment. 


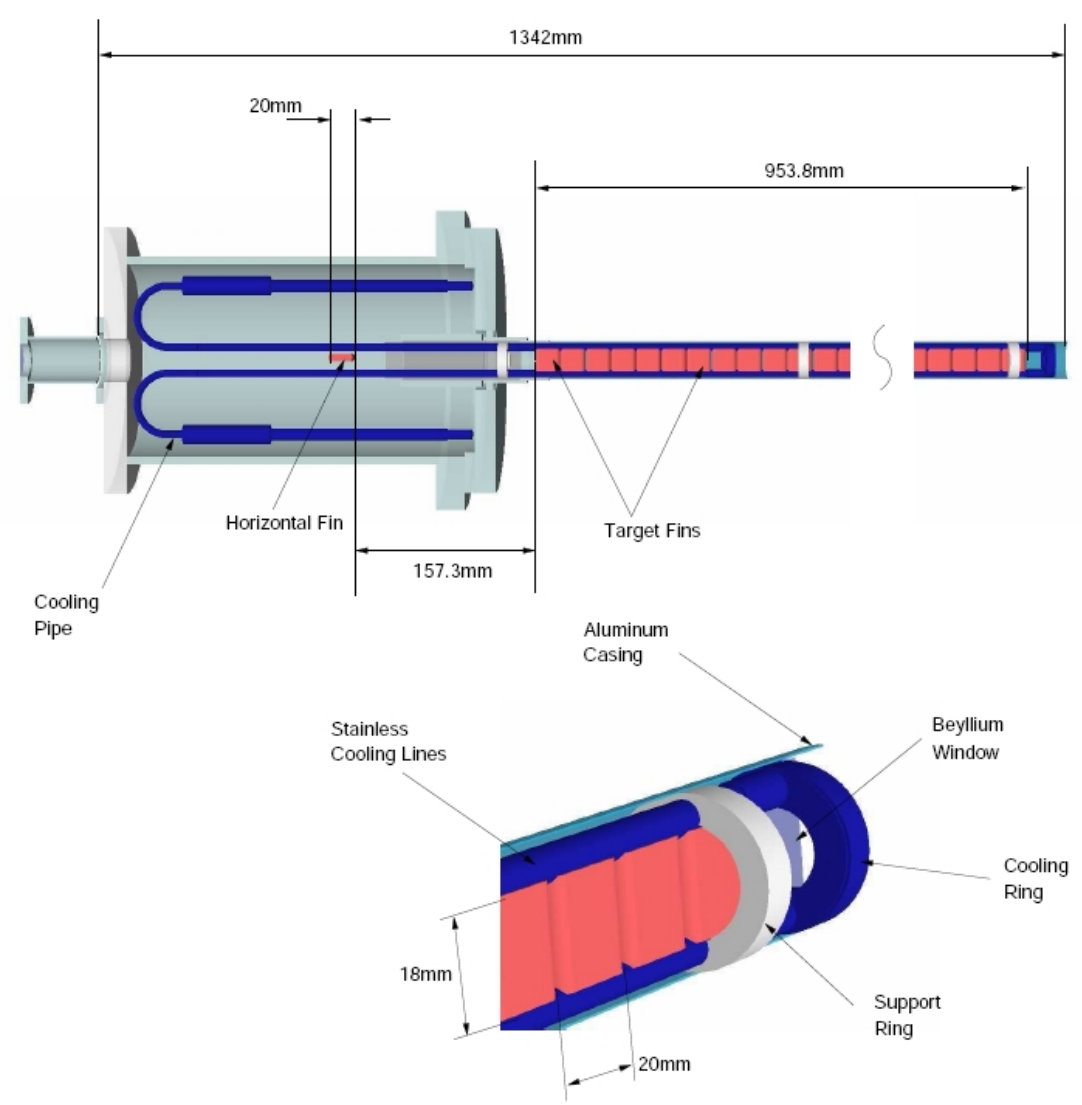

Figure 3.2: The NuMI target which is composed of 47 separate graphite segments. The segments are contained in a Aluminum container and cooled by circulating water in cooling pipes. The Aluminum container has Beryllium windows at the upstream and downstream ends. Taken from [45].

Circulating water cools the the graphite segments and the entire system is enclosed in an aluminum vessel. The beam spot size at the target is $1.2-1.5 \mathrm{~mm}$.

The total dimensions of the target are $6.4 \mathrm{~mm}$ in width, $15 \mathrm{~mm}$ in height and $940 \mathrm{~mm}$ in length. This length corresponds to about 2 interaction lengths, therefore most of the protons will interact in the target. The target has a collimating baffle that protects the target and other downstream components from stray particles. An advantage of the segmented design is that it allows for small-angle, high momentum secondaries to escape the target with a small chance of re-interacting. 


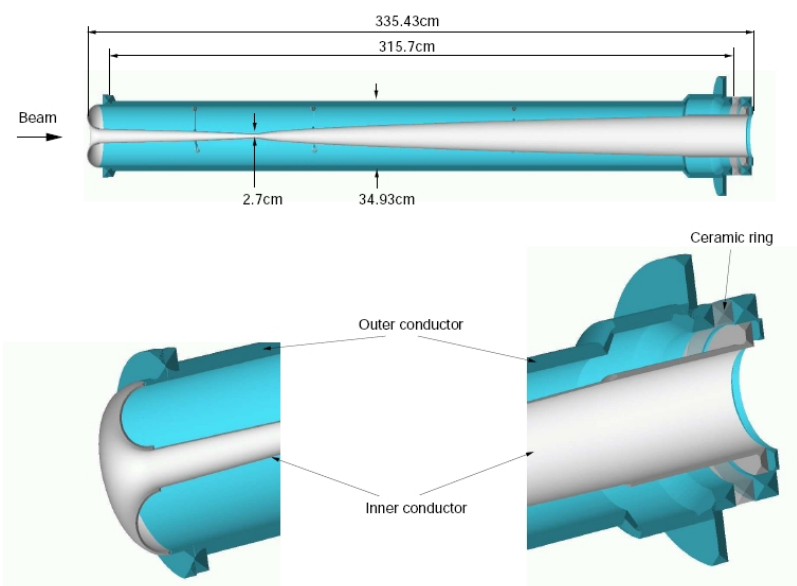

Figure 3.3: The first horn of the NuMI beam. During beam operation the current is pulsed down the inner conductor creating a toroidal magnetic field. Hadrons that pass in the region between will have their trajectories altered. Taken from [45].

\subsubsection{Focusing Horns}

The focusing of pions and kaons (hadrons) is essential to increase the neutrino flux. Focusing changes the outgoing direction of the hadrons produced off the target and directs them towards the detectors. NuMI employs two magnetic horns separated by 10 m. Each horn consists of two aluminum conductors, symmetric about the beam axis (see Figures 3.3 and 3.4). A current is pulsed down the inner conductor and returned through the outer conductor thereby producing a toroidal magnetic field in between. The outer conductor is $2.54 \mathrm{~cm}$ thick while the inner conductor only a few millimeters to reduce scattering. The shape of the inner conductor is parabolic giving particles entering at larger radius a greater change in momentum.

During beam spills the horns are pulsed simultaneously with current up to $200 \mathrm{kA}$ producing a maximum $30 \mathrm{kG}$ field. The absolute value of the current was calibrated to within $0.5 \%$ and was seen to vary by $0.2 \%$ over the course of data collection. Alignment of the target horn system is done using the beam directly.

Figure 3.5 shows 5 different trajectories through the two horn system, and the predicted energy spectrum for each of those trajectories at the Near Detector. A majority 


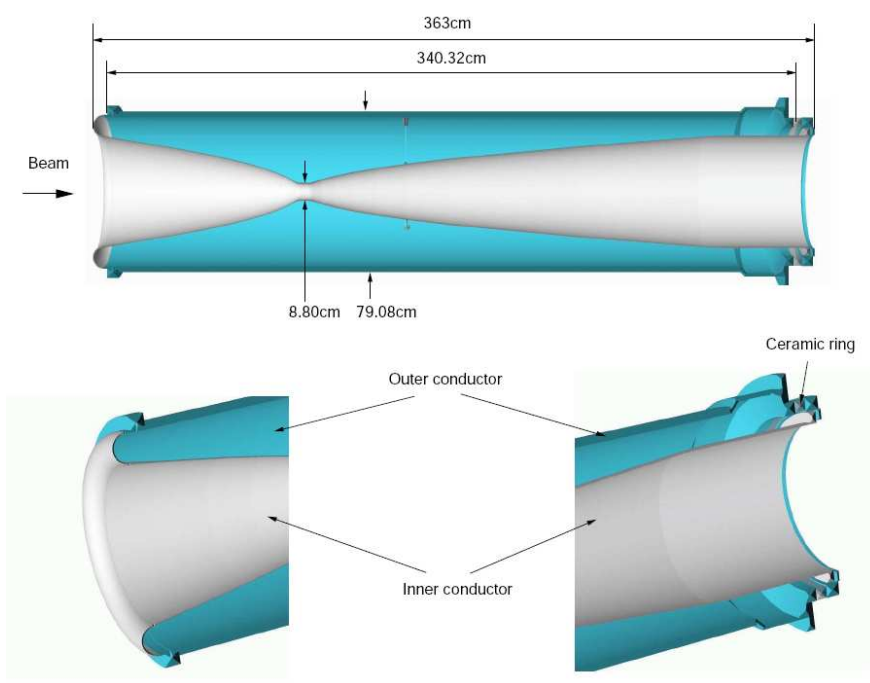

Figure 3.4: The second horn of the NuMI beam. During beam operation the current is pulsed down the inner conductor creating a toroidal magnetic field. Hadrons that pass in the region between will have their trajectories altered. Taken from [45].

of the neutrino events in the peak are from hadrons that are "Overfocused" while the high energy neutrinos originate from hadrons that pass through the aperture (neck) of the horns and thus are not subject to any focusing.

\subsubsection{Decay Volume}

The decay volume is a steel pipe $2 \mathrm{~m}$ diameter and $675 \mathrm{~m}$ long. The pipe has been evacuated to 0.5 Torr to reduce absorption and scattering. An upstream window was designed out of Aluminum to maintain structural integrity under vacuum while minimizing scattering. The upstream window is made of $1.6 \mathrm{~mm}$ thick aluminum and the downstream of steel $4.76 \mathrm{~mm}$ thick. The length of the decay pipe is determined by the average distance that pions of energy 5 to $10 \mathrm{GeV}$ will travel. The decay length for a $10 \mathrm{GeV}$ pion is $\gamma \beta c \tau=560 \mathrm{~m}$

Particles that do not decay in the decay volume will be absorbed by the hadron absorber. The absorber consists of a cooled aluminum core surrounded by steel and concrete blocks. The muons will pass through and be be absorbed in the intervening rock upstream of the Near Detector. 

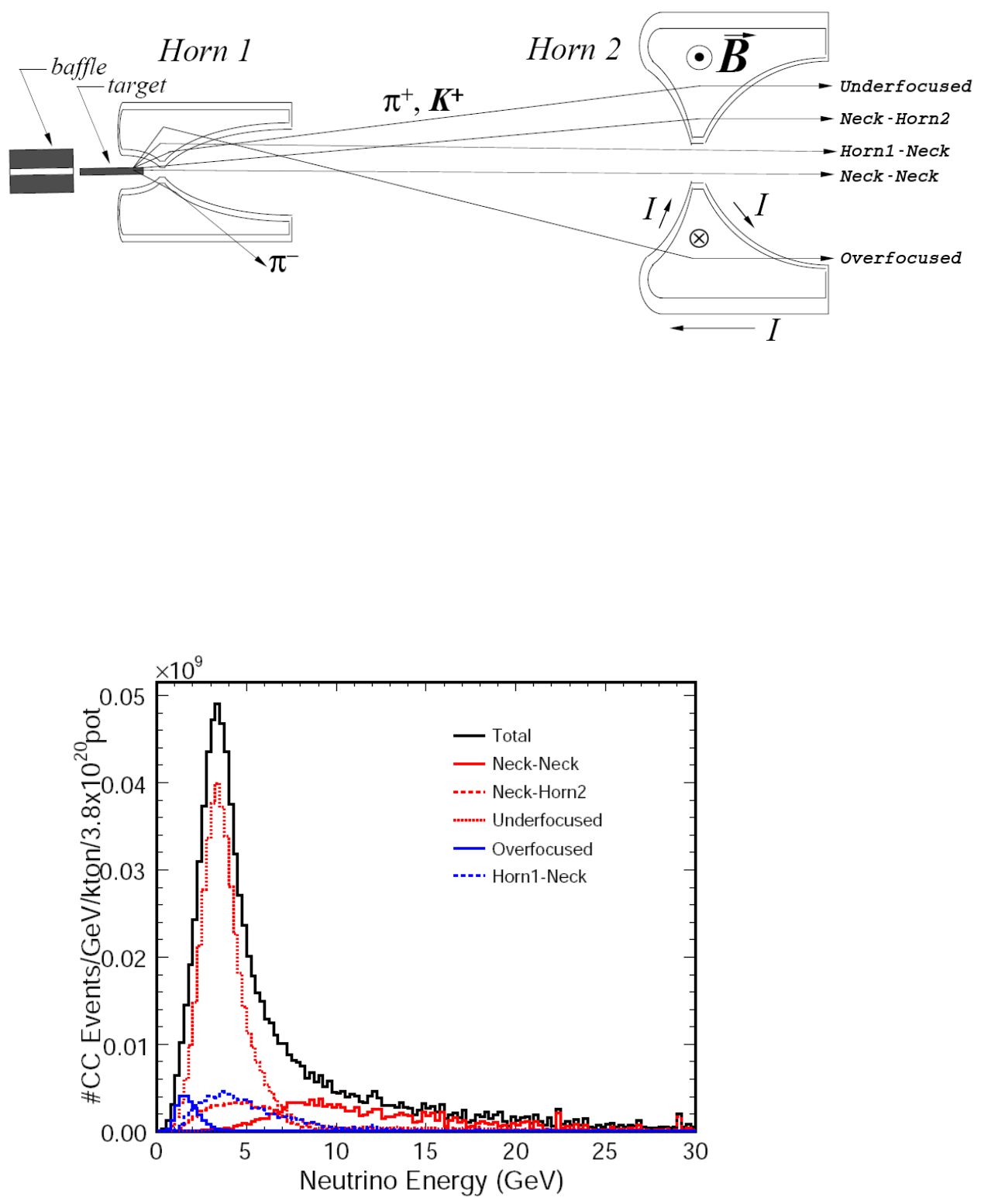

Figure 3.5: Top: A schematic of the NuMI beamline with five different possible trajectories for a positive hadron (the negative hadrons will be focused away) focused through the two horn system. Bottom: The corresponding neutrino energy spectrum at the Near Detector for the five different trajectories. "Neck-Neck" refers to hadrons that are unfocused and pass through the neck of both horns, "Horn1-Neck" and "Neck-Horn2" refer to hadrons focused through only one of the horns, "Underfocused" refers to hadrons that receive little change from horn 1 , and "Overfocused" are those that get a large correction from horn 1 and over-corrected by horn 2. Taken from [45] 


\subsubsection{Different Beam Configurations}

A unique feature of the NuMI beam is that by varying the beamline configuration the average neutrino energy can be changed. The target and baffle are mounted on a rail system that shifts the position of the target. As the target moves, the relative distance between the target and horns changes. This distance selects the longitudinal momentum, or $p_{Z}$, of the hadrons that will be focused. When the target is closer to the horns, hadrons with lower $p_{Z}$ are focused. The current controls the transverse momentum, or $p_{T}$, of the focused hadrons.

Seven different combinations of target position and horn current have been used in data taking. Figures 3.6 to 3.9 illustrate the momentum of the hadrons that are focused for different configurations. They show the probability that a $\nu_{\mu} / \bar{\nu}_{\mu}$ from $\pi / K$ decays will interact in the Near Detector as a function of $p_{T}$ and $p_{Z}$. As the momenta of the selected parent hadrons changes so will the typical neutrino energy. In contrast to the neutrinos, the antineutrinos are defocused away from the MINOS detectors. The changing of the beam configuration does not affect the selection of these hadrons and therefore the antineutrino flux.

Different configurations are labeled by the string leXXXzYYYi, where "le" indicates the $10 \mathrm{~m}$ separation of the two horns, $\mathrm{XXX}$ is the distance from the target to the horns in $\mathrm{cm}$ (along the beam axis) and YYY is the horn current in kA. Table 3.1 lists the seven different configurations and the target position and horn current. Several configurations also have shorter labels that will frequently be used. Figure 3.10 compares the neutrino energy spectra at the Near Detector for the LE, ME, and HE configurations.

Below $30 \mathrm{GeV}$, a majority of neutrinos come from pion decay while kaons dominate above. Figures 3.11 and 3.12 show the relative contributions of the flux from different parent hadrons in four different beam configurations: Horn Off, LE, ME, and HE.

The greatest sensitivity to oscillations is found when running in the LE configuration, where the typical neutrino energy is the lowest. Most of the time MINOS has spent 

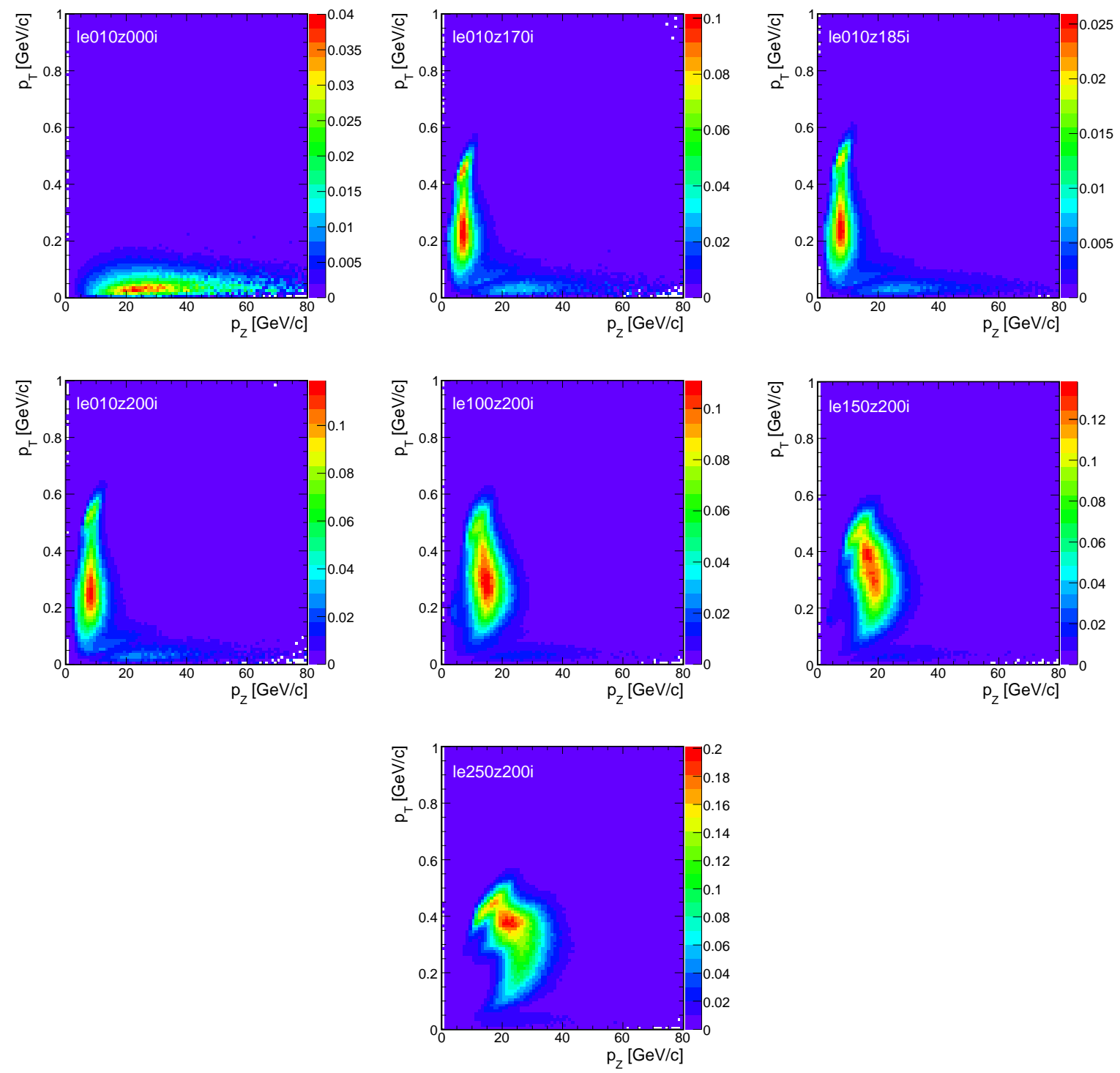

Figure 3.6: The probability that a $\pi^{+}$will make a charged current $\nu_{\mu}$ event that is detected in the Near Detector as a function of $p_{T}$ and $p_{Z}$. 

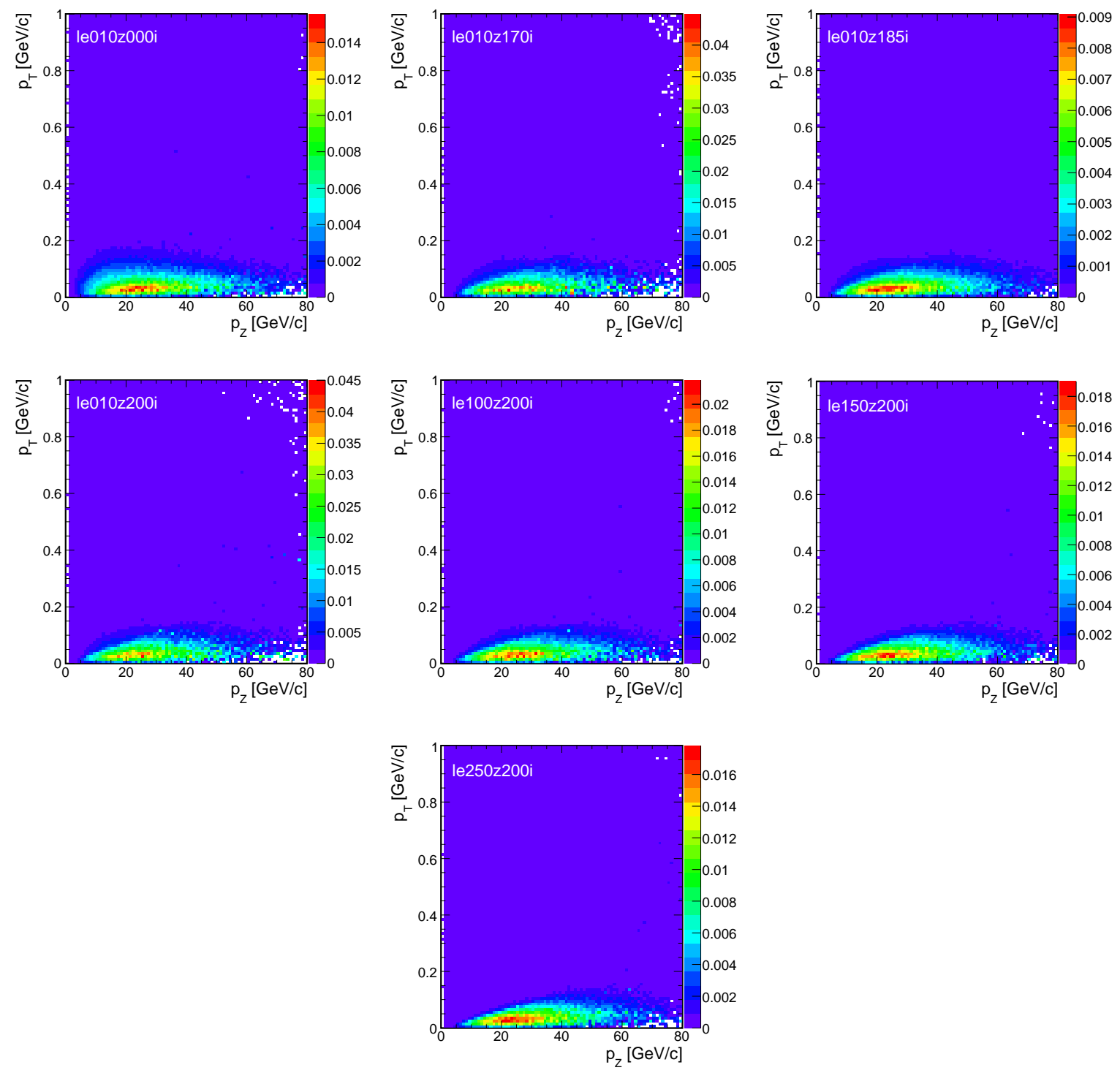

Figure 3.7: The probability that a $\pi^{-}$will make a charged current $\bar{\nu}_{\mu}$ that is detected in the Near Detector as a function of $p_{T}$ and $p_{Z}$. 

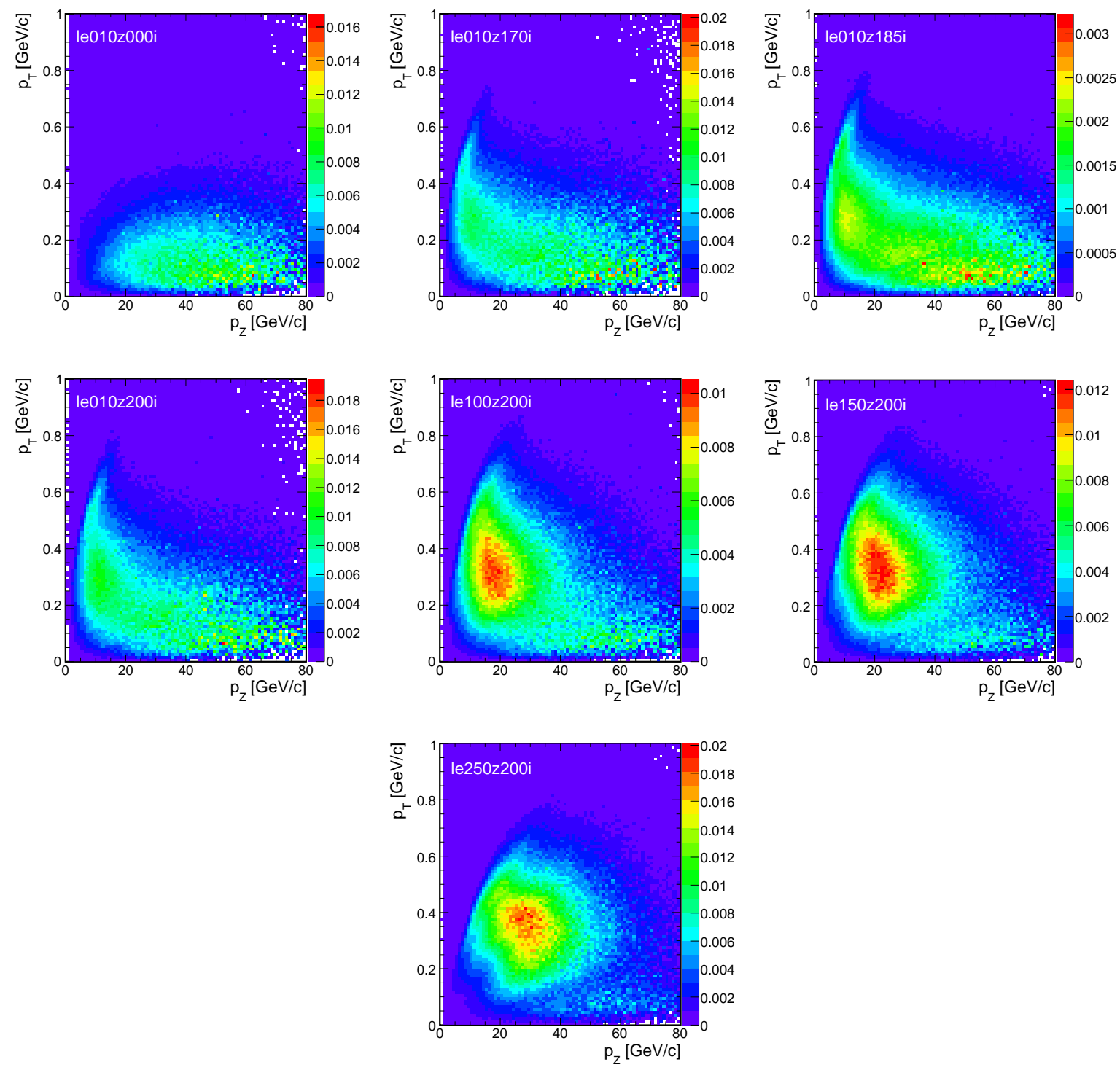

Figure 3.8: The probability that a $K^{+}$will make a charged current $\nu_{\mu}$ that is detected in the Near Detector as a function of $p_{T}$ and $p_{Z}$. 

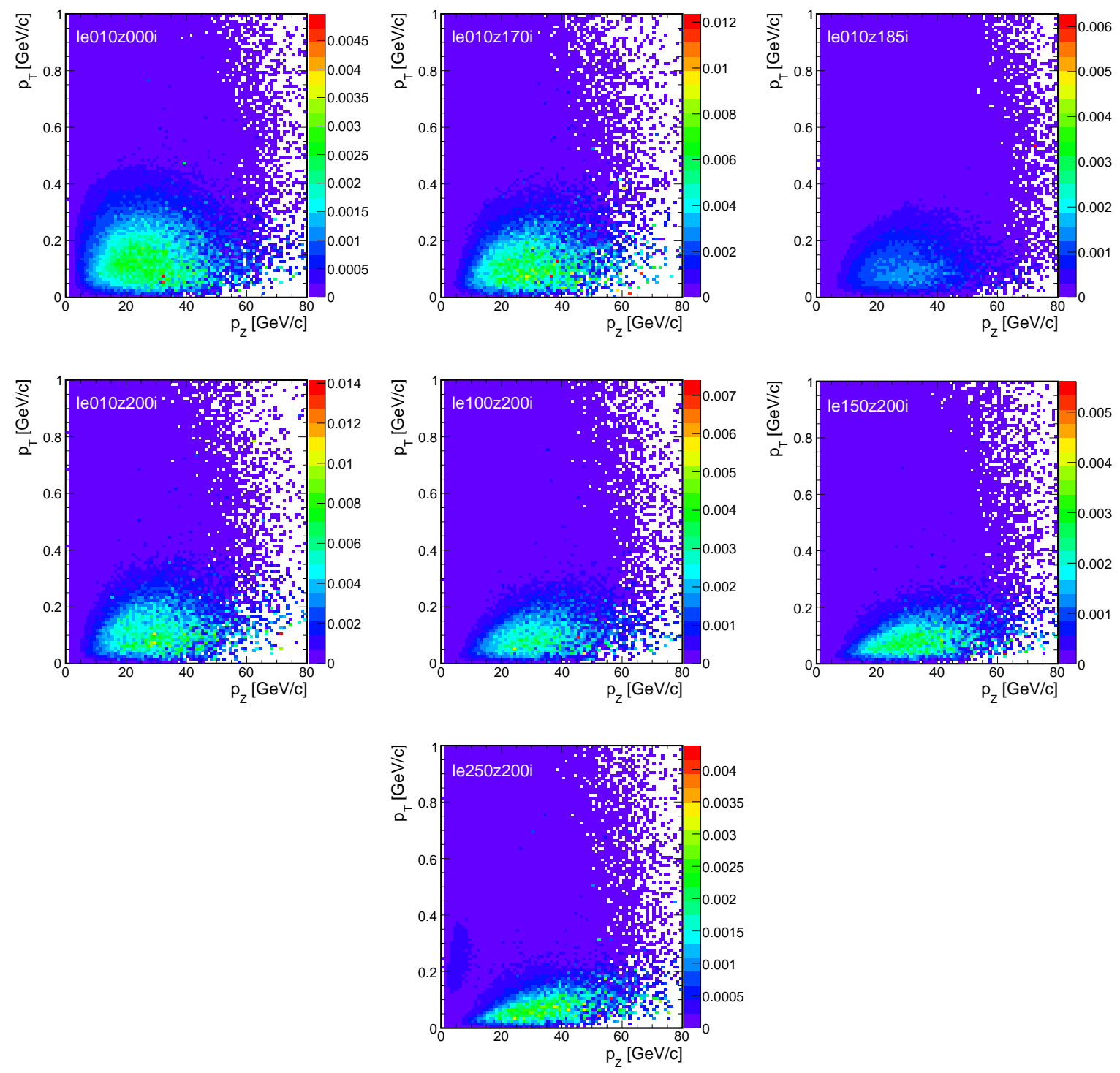

Figure 3.9: The probability that a $K^{-}$will make a charged current $\bar{\nu}_{\mu}$ that is detected in the Near Detector as a function of $p_{T}$ and $p_{Z}$. 


\begin{tabular}{lccl}
\hline \hline Beam Label & Target Position & Horn Current & Short Label \\
\hline le010z000i & $10 \mathrm{~cm}$ & $0 \mathrm{kA}$ & Horn Off \\
le010z170i & $10 \mathrm{~cm}$ & $170 \mathrm{kA}$ & None \\
le010z185i & $10 \mathrm{~cm}$ & $185 \mathrm{kA}$ & Low Energy (LE) \\
le010z200i & $10 \mathrm{~cm}$ & $200 \mathrm{kA}$ & None \\
le100z200i & $100 \mathrm{~cm}$ & $200 \mathrm{kA}$ & Medium Energy (ME) \\
le150z200i & $150 \mathrm{~cm}$ & $200 \mathrm{kA}$ & Medium High Energy (MHE) \\
le250z200i & $250 \mathrm{~cm}$ & $200 \mathrm{kA}$ & High Energy (HE) \\
\hline \hline
\end{tabular}

Table 3.1: Beam configurations that have been used to collect data in MINOS. The target position refers to the distance upstream of the default position where the target is inserted fully into horn 1.

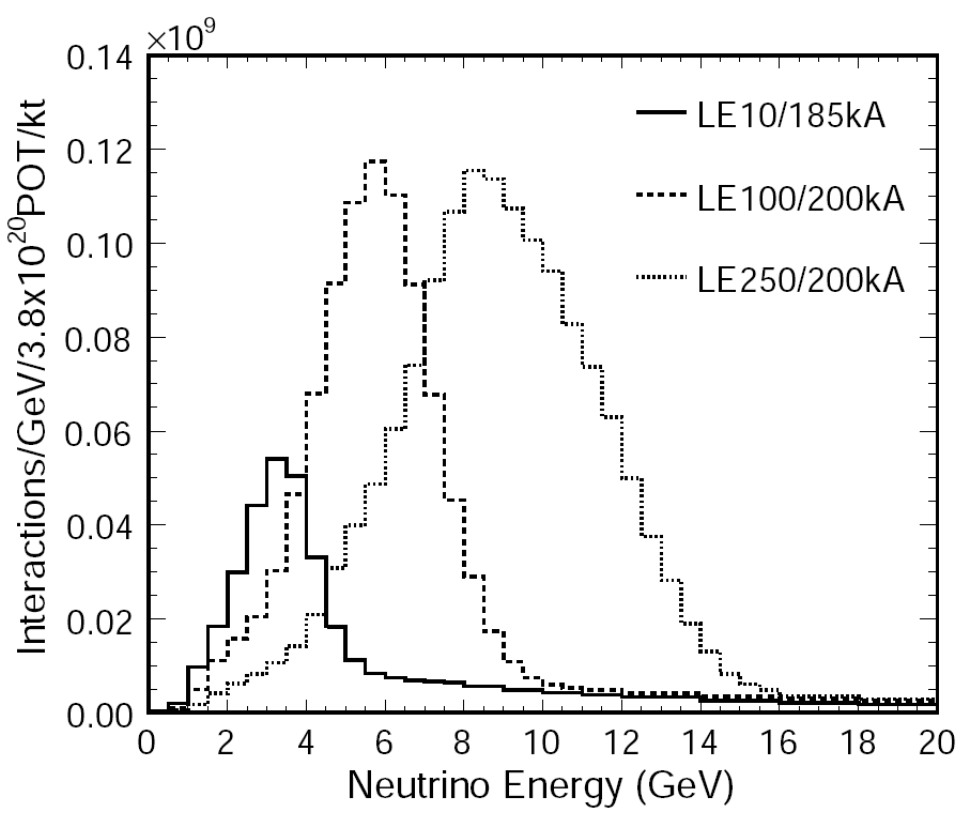

Figure 3.10: A Monte Carlo simulation of the total $\nu_{\mu}$ spectra for three beam configurations: le010z185i (LE), le100z150i (ME), and le250z200i (HE). Taken from [46]. 

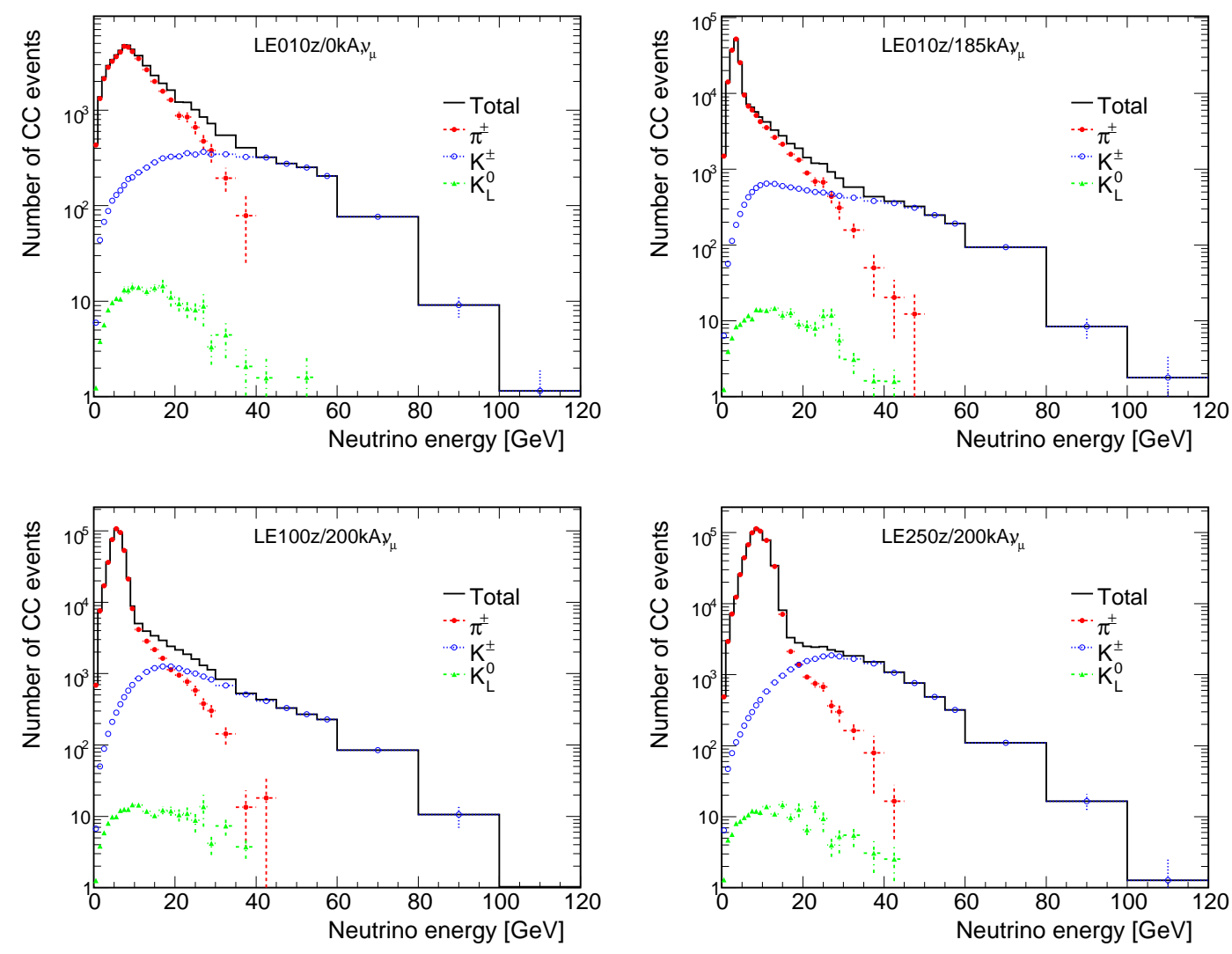

Figure 3.11: Monte Carlo $\nu_{\mu}$ CC spectra in the Near Detector for the le010z000i(upper left), le010z185i(upper right), le100z200i(lower left), le250z200i(lower right) configurations. The individual components from charged and neutral kaons and pions are indicated as well. Taken from [47]. 

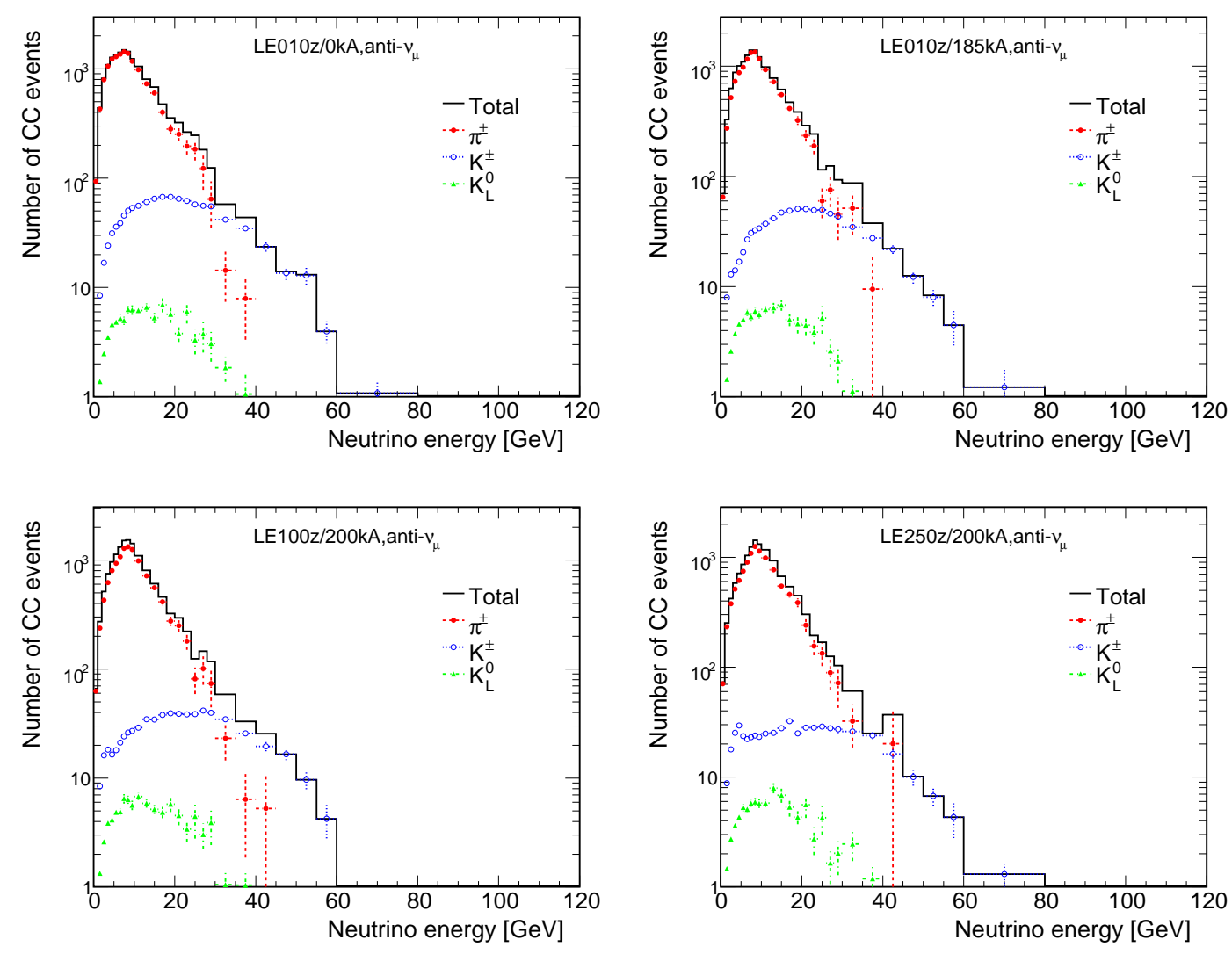

Figure 3.12: Monte Carlo $\bar{\nu}_{\mu}$ CC spectra in the Near Detector for the le010z000i(upper left), le010z185i(upper right), le100z200i(lower left), le250z200i(lower right). The individual components from charged and neutral kaons and pions are indicated as well. Taken from $[47]$. 
running in this configuration. The other beams have been used to constrain the physics of hadrons production from the target (see Chapter 5).

\subsubsection{Uncertainties on the $\nu_{\mu}$ Flux}

Incorrect alignment or modeling of the focusing will change the neutrino energy spec-

trum. Uncertainties from these effects can be readily calculated by the Monte Carlo (see Section 4.3). They also have the advantage of being constrained by external data from the beam monitoring instrumentation. Figure 3.13 shows the estimated $1 \sigma$ uncertainties as a function of neutrino energy for: the alignment of the horn and target, the modeling of the current in the horns, the fraction of the beam scraping on the upstream shielding components, and the uncertainty on the number of protons on target.

These figures show that the uncertainty on the ratio of the event yield of the Far/Near is less than that of the Near alone. At high energies the uncertainty is small because the parent hadrons are not focused by the horns. The largest uncertainty is seen at $\sim 5 \mathrm{GeV}$ where the hadrons cross the edges of the focusing horn apertures.

\subsection{The MINOS Detectors}

MINOS uses two functionally identical detectors to measure neutrinos. The Near Detector measures the unoscillated neutrino spectrum and the Far Detector is used to search for signs of oscillations. Both detectors are magnetized steel-scintillator sampling calorimeters. They consist of alternating planes of plastic scintillator strips and steel $2.54 \mathrm{~cm}$ thick. The scintillator is made of $1 \mathrm{~cm}$ thick $\times 4.1 \mathrm{~cm}$ wide strips of polystyrene coextruded with a $\mathrm{TiO}_{2}$ reflective coating. Down the center of each strip a wavelength shifting fiber is glued to collect the light. The wavelength-shifting (WLS) fibers transport the light to optical connectors located on the edge of the scintillator where it is then transferred to a clear polystyrene fiber and routed to photomultiplier tubes (PMTs) as shown in Figure 3.14. 

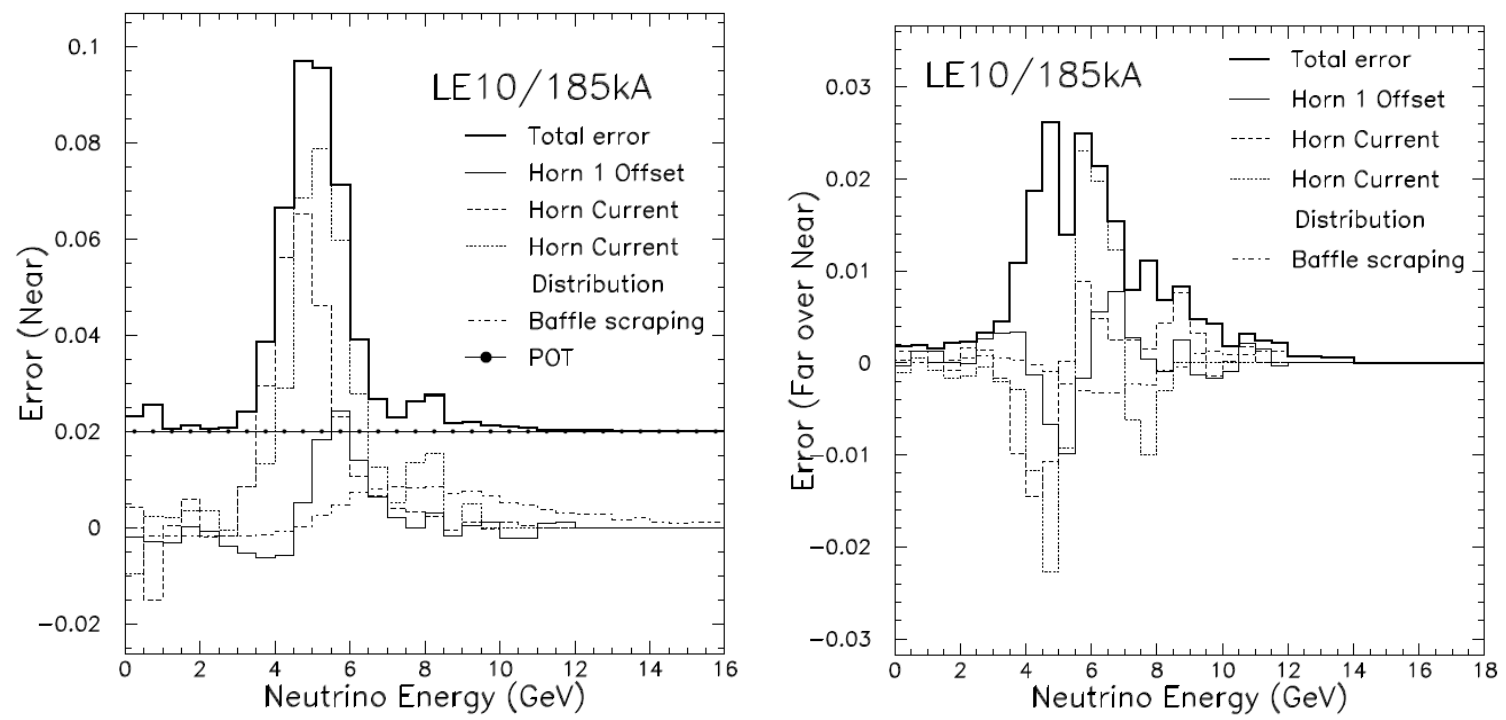

Figure 3.13: The flux uncertainties on the $\nu_{\mu}$ flux as a result of beam alignment and focusing. The lines show the fractional change in the number of neutrino events as a function energy for a $1 \sigma$ shift in various beam parameters; these include the position of the horn and target, the modeling of the current and the effects of the upstream baffle. The left shows the effect on the Near Detector spectrum and the right shows the effect on the Far/Near ratio. Taken from [46].

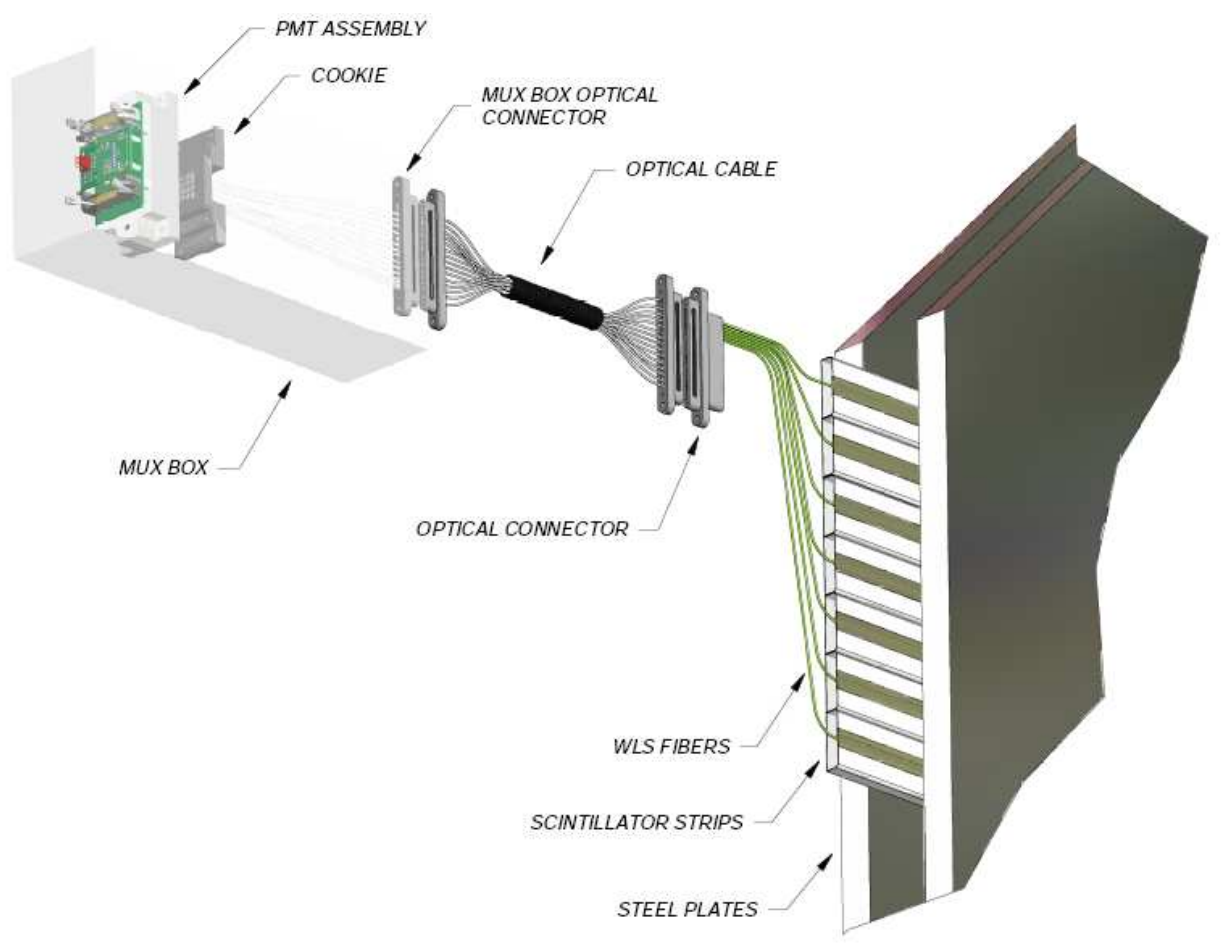

Figure 3.14: Drawing of the scintillator readout system. WLS Fibers from the scintillator are carried to clear optical fibers and routed to PMTs. Taken from [48]. 
Each plane has one "view" of strips with the next oriented $90^{\circ}$ to the previous. The two views (known as $u$, and $v$ ), are oriented $45^{\circ}$ to the horizontal and provide 3-D topology.

\subsubsection{Near Detector}

The Near Detector (see Figure 3.15) is located 100 m (225 mwe) underground at Fermi National Laboratory in Batavia, Illinois. The detector is divided into two separate regions. The first 121 planes make up the calorimeter. One in five planes are fully instrumented with scintillator strips; the others are only partially instrumented. The calorimeter contains the hadronic showers of the events. The last 161 planes comprise the spectrometer. The spectrometer saves on scintillator by reducing the channel count so only one in every in five is instrumented. This saving comes at the cost of some ambiguity in event reconstruction. Four separate channels share PMTs and are electronically summed. The spectrometer is designed to track the muons created in the calorimeter

Fully instrumented planes have 96 scintillator strips, while the partially instrumented planes have 64. Modules are formed by grouping scintillator strips side-by-side and sandwiching them between aluminum covers. Figure 3.16 shows the layout of different strip patterns for partially and fully instrumented planes and for the different views. The 282 total planes amount to $0.98 \mathrm{kT}$.

For the Near Detector, the steel planes are "squashed-octagons" with dimensions $6.2 \mathrm{~m}$ wide and $3.8 \mathrm{~m}$ high. Each plane has a hole $30 \times 30 \mathrm{~cm}^{2}, 59 \mathrm{~cm}$ from the center of the plane where magnetic coil resides. The coil hole is outside of the fiducial region of the beam. The magnetic field is generated by $40 \mathrm{kA}$ of current producing a $1.17 \mathrm{~T}$ field at the center of the beam spot.

During normal beam operations, an average of 15 neutrino events per spill are recorded. Fast timing resolution of $20 \mathrm{~ns}$ is needed to separate out individual events. A typical beam spill in the Near Detector is shown in Figure 3.17. 

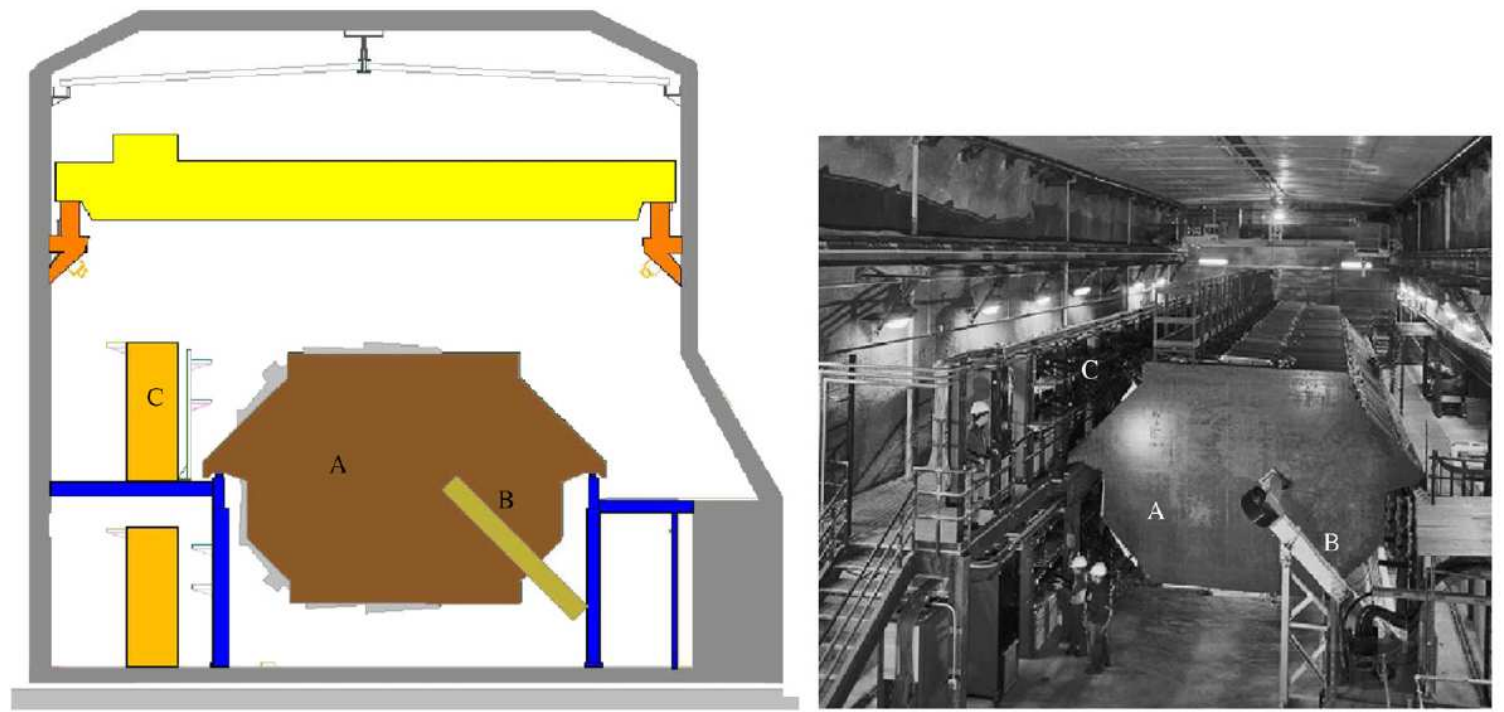

Figure 3.15: Top Left: End view of the Near Detector looking towards Soudan. "A" is the point where the NuMI beam hits the detector , "B", indicates the magnetic coil and "C" shows the electronics rack that are read out on one side of the Near Detector. Top Right: An actual picture of the Near Detector with the same points labeled. Taken from [49].
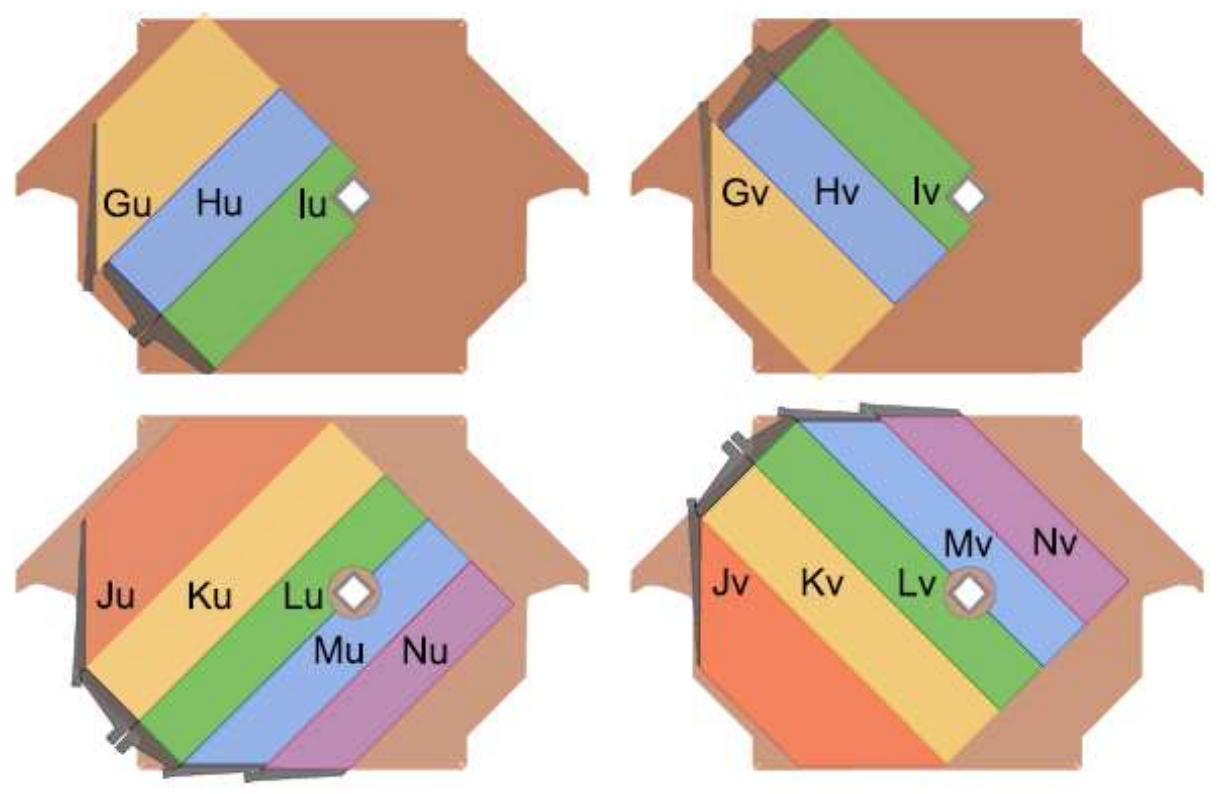

Figure 3.16:

The four different types of planes used in the Near Detector. The two upper planes show the partially instrumented planes with the left for the $u$ view and the right for the $v$. The lower two planes show the fully instrumented planes for $u$ (left) and $v$ (right).

The G-N labels denote different scintillator modules. Taken from [49]. 


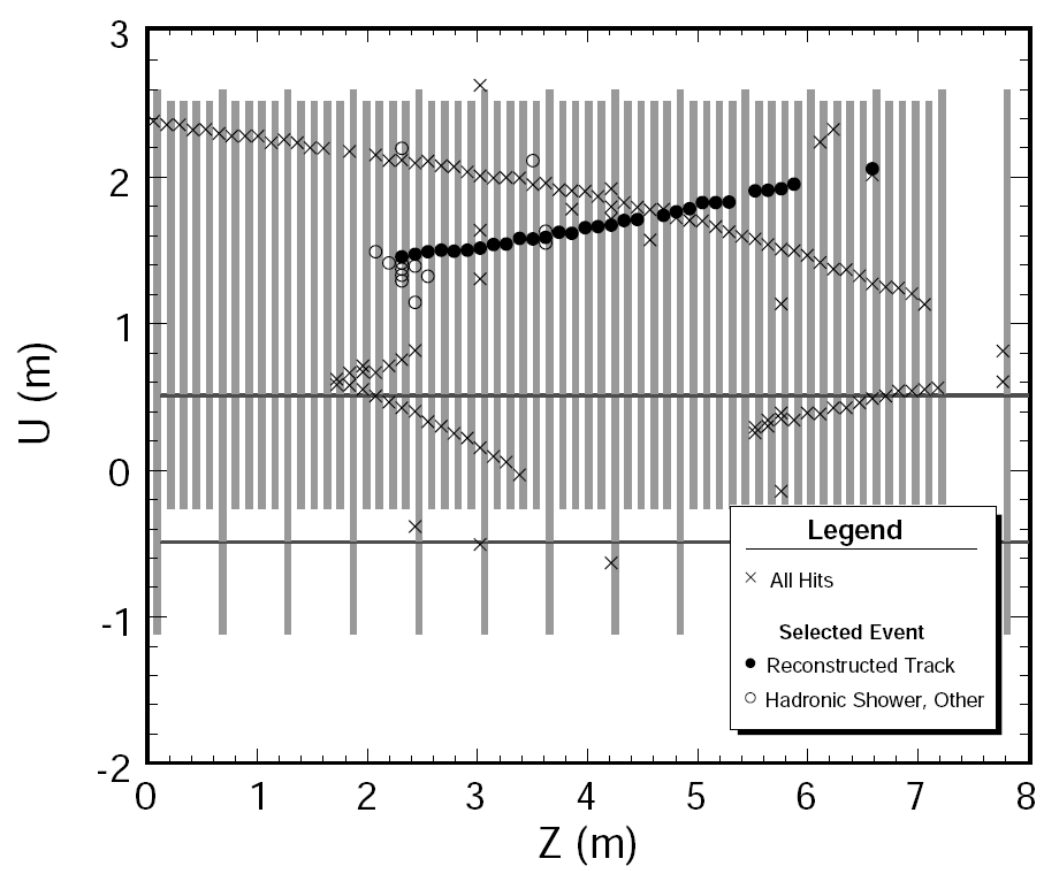

(a)

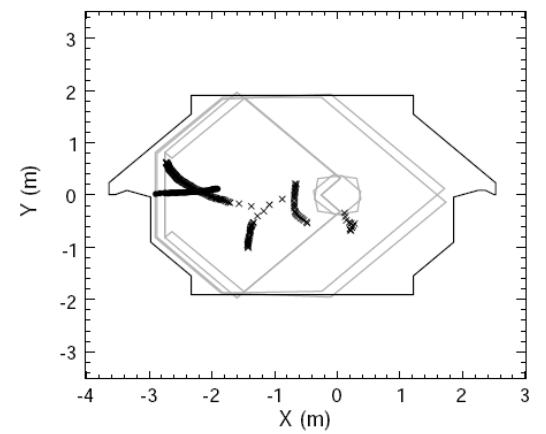

(b)

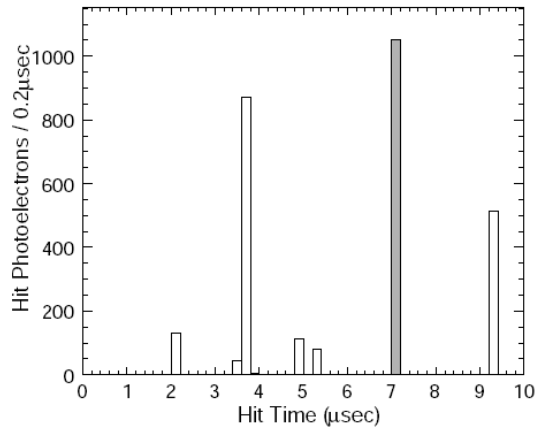

(c)

Figure 3.17: One beam spill in the Near Detector. Top: The $u$ planes with markers to indicate hits in the detector. The beam is incident on the left side and the coil hole is seen at the center of the graph. Bottom Right: A horizontal and vertical view of the hits. Bottom Left: The hits as a function of time. The bin width is about ten times larger than the resolution. Taken from [46] 
The electronics in the Near Detector is governed by the much higher instantaneous event rate than the Far Detector. During the $10 \mu$ s spill there must be no dead time in order to record all of the events. Fast electronics are required with the speed set by the $53 \mathrm{MHz}$ RF frequency of the Main Injector. The readout is based on a custom integrated circuit designed at Fermilab called the Charge Integrated Circuit (QIE) [49].

The triggering is done in two modes: Spill and Cosmic Rays. A signal from the Main Injector initiates an acquisition period of up to $19 \mu$. Data is collected for the entire spill and stored in FIFO front-end cards. After the spill, the data is readout and individual events are time-stamped according to the RF bucket with which they were associated. In cosmic ray mode, data is collected during the time in between spills. The last dynode of each PMT initiates a 150 ns readout period used to record the cosmic rays.

\subsubsection{Far Detector}

The Far Detector (see Figure 3.18) was constructed in an inactive iron mine in Soudan, Minnesota and consists of 486 planes of steel. The planes are $8 \mathrm{~m}$ wide regular octagons. The planes are separated into two "supermodules" separated by $1.15 \mathrm{~m}$. The first supermodule number has 249 steel planes and the second 237. The first upstream plane of each supermodule does not have a corresponding scintillator plane, resulting in 484 total scintillator planes. All planes are fully instrumented with a total of 192 scintillator strips per plane for a total mass of $5.4 \mathrm{kT}$. Unlike the Near Detector, the scintillator strips are read out from both sides of the detector. Through the center is routed a $15.2 \mathrm{kA}$ magnetic coil producing an average field of $1.2 \mathrm{~T}$.

The optical signals are recorded by 16-anode (M16) Hamamatsu PMTs. The signal from eight different fibers are optically summed to reduce the PMT cost. The process of choosing the correct arrangement of hits is done in the offline software. To distinguish between upward and downward going muons the timing resolution is 3-5 ns [49]. 

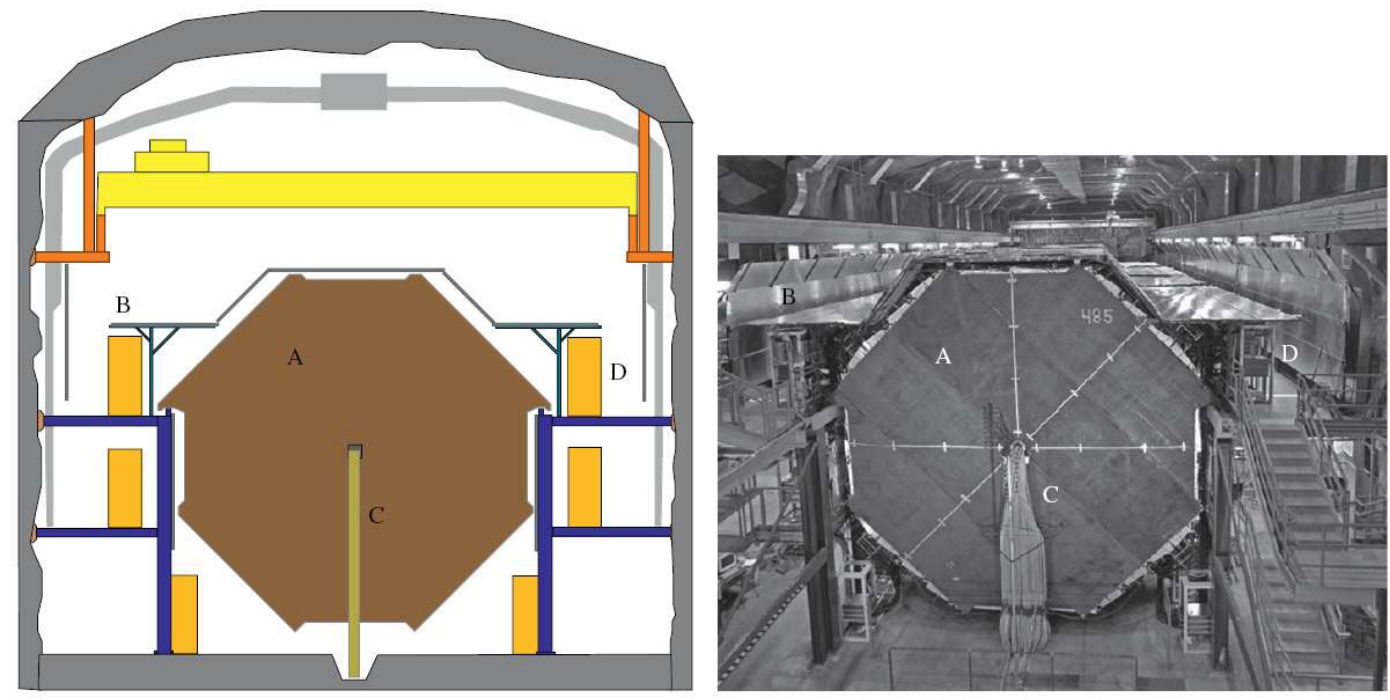

Figure 3.18: Left: End view of the Far Detector looking towards Fermilab. "A" is the last steel plane of the second supermodule, "B", indicates the veto shield, "C" shows the magnetic coil and "D" shows the electronics racks that are read out on both sides of the Far Detector. Right: An actual picture of the Far Detector with the same points labeled. Taken from [49]

The electronics in the Far Detector were designed to handle the low rate seen in an underground environment. The number of neutrino events from NuMI is only a couple per day. The signal rate is therefore dominated by the detector noise. The data is digitized by a 14-bit ADC and transferred to a FIFO for processing. Since a direct spill signal is not possible at the Far Detector, a GPS timing system at the Near Detector generates timestamps of the spill signals. These are transmitted over the Internet to the Far Detector. Taking into account corrections for antenna delays, hardware offsets and 2.4 ms to travel from Fermilab to Soudan, the data around a window of the spill trigger is written out as a beam event. The buffering capability allows for ample time to wait for spill information. When spill information is not available cosmic ray triggering is done via algorithms based on clustering hits. 


\subsubsection{Calibration Detector}

The Calibration Detector was a much smaller prototype detector used to calibrate the response to individual particles. It consisted of 60 of $1 \times 1 \mathrm{~cm}^{2}$ planes for a total of 12 tons. The Calibration Detector was inserted into the CERN PS test lines and exposed to pions, electrons, muons and protons with momenta from $0.2-10 \mathrm{GeV}$. It was instrumented with both Near and Far electronics. Unlike the Near and Far Detectors the Calibration Detector didn't need to be magnetized since the momentum of the test particles was known. The measurements carried out there were used to validate the MINOS Monte Carlo simulations and to estimate the uncertainty on the shower or hadronic energy scale.

The beam ran for different polarities of the mentioned particle types. Time-of-flight and Cerenkov detectors were used to distinguish particle types The data was compared to the same GEANT3 based Monte Carlo used for the Near and Far detectors. Figure 3.19 shows the detector response to electrons for three different momentum settings. The electron data agree with the Monte Carlo to $2 \%$ [50] and the pion and proton shower response to $6 \%$ [51]. These results provide the absolute hadronic scale uncertainty. 


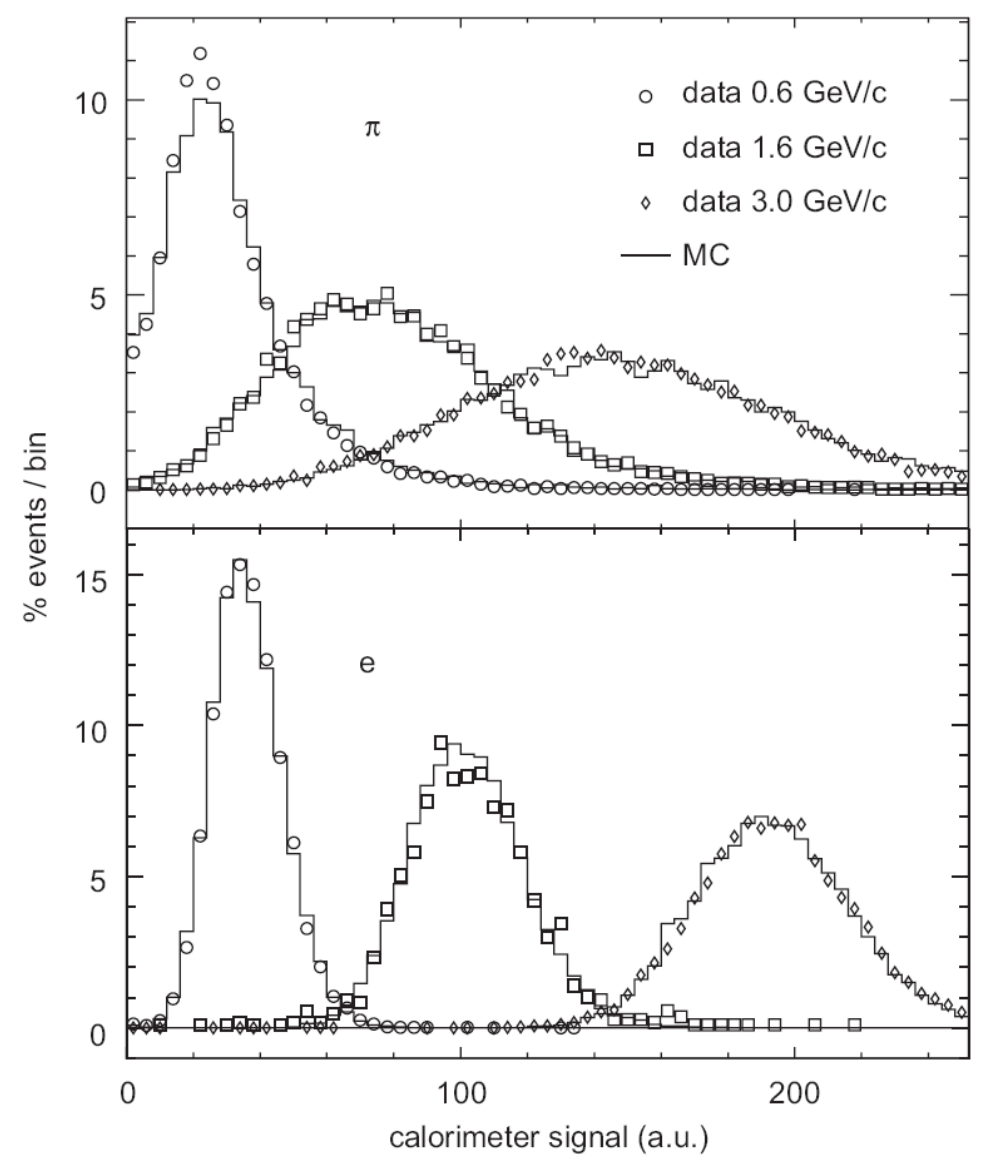

Figure 3.19: Calorimetric response at the Calibration Detector for pions (top) and electrons (bottom) at three different beam energies for data (circles) and Monte Carlo (solid line). The x-axis scale is in arbitrary units. Taken from [49]. 


\section{Chapter 4}

\section{Detector Operations and Simulations}

The NuMI beam first turned on in May 2005 and continues to take data. The data is divided into three separate run periods separated by shutdowns of the accelerator complex. RunI spans from May 2005-February 2006, RunII from June 2006-June 2007 and RunIII October 2007-June 2009. Only data from RunI and RunII are considered in this thesis. Figure 4.1 gives the number of protons on target (POT) delivered to MINOS as a function of time for RunI and RunII. Table 4.1 lists the total amount of reconstructed data from the Near Detector in each beam configuration.

Figure 4.2 gives examples of CC and NC events as viewed in MINOS. CC interactions produce a muon which will penetrate many planes before ranging out after steady ionization losses. NC events appear as a collection of hits emanating from the interaction vertex. Identification of charged current events is important for the oscillation analysis and will be discussed further in Chapter 7 .

\subsection{Reconstruction Algorithms}

The MINOS event reconstruction software was developed over several years by many people. It analyzes collections of hits, separating them into individual neutrino events of tracks and shower. The software assigns a total neutrino energy to each event based 


\begin{tabular}{lc}
\hline \hline Beam Configuration & POT $\left(\times 10^{18}\right)$ \\
\hline le010z185i RunII & 194.0 \\
le010z185i RunI & 125.9 \\
le250z200i RunII & 15.4 \\
le010z000i RunII & 2.8 \\
le010z000i RunI & 2.6 \\
le250z200i RunI & 1.6 \\
le150z200i RunII & 1.6 \\
le010z170i RunI & 1.4 \\
le010z200i RunI & 1.4 \\
le100z200i RunI & 1.1 \\
\hline \hline
\end{tabular}

Table 4.1: The total amount of data from the Near Detector in each beam configuration measured in protons on target (POT). The beam configurations are labeled following the convention defined in Section 3.1.5.

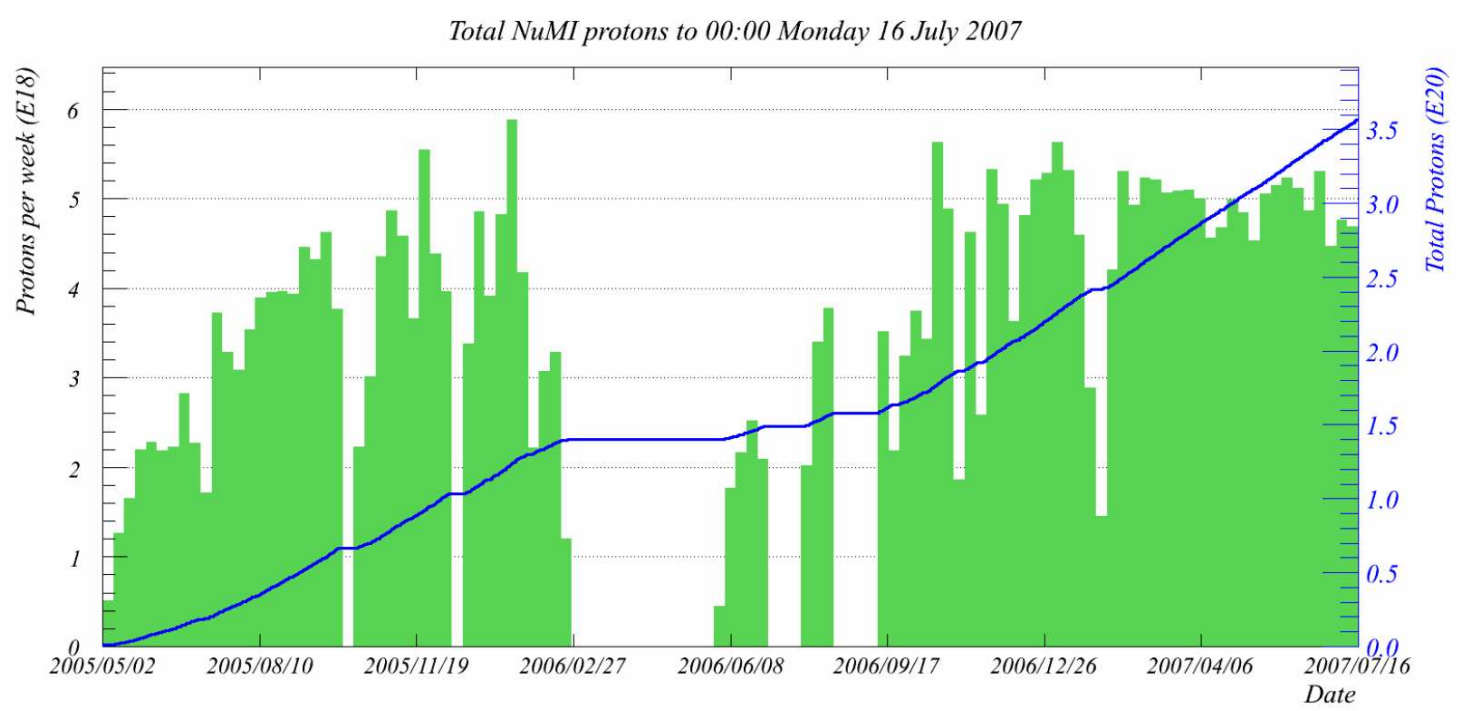

Figure 4.1: The number of POT accumulated from the NuMI beamline. The green histogram indicates the weekly amount with the axis on the left, and the blue line is the total with the axis on the right. Taken from [52] 


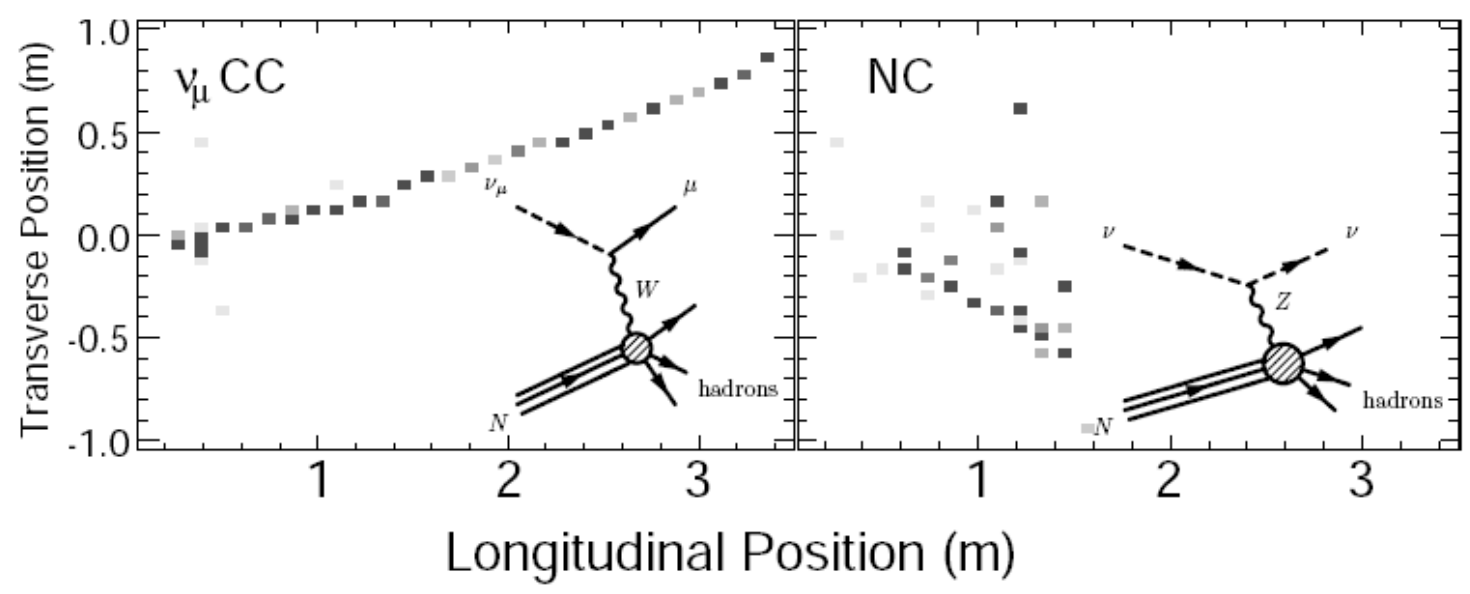

Figure 4.2: Left: One view of a CC neutrino interaction in the MINOS detector. Right: One view of a NC interaction. Shaded areas indicate the amount of energy deposited in individual scintillator strips with darker shades signifying more energy. Taken from [48].

on the sum of track and shower energies. The formation of neutrino events is done as follows:

i. Separating individual neutrino events - In the Near Detector up to 20 neutrino events can be in one spill. Individual events can be separated by localization in space and time. The Far Detector, in general, only has one neutrino event per spill and this step is not necessary.

ii. Demuxing - In the Far Detector (and the spectrometer in the Near Detector) multiple scintillator strips share the same PMT. The process of choosing the correct combination of hits associated with detector positions is known as "demuxing" [53]. In the Far Detector different patterns are used for each side of the detector to provide a better reconstruction of particles. The pattern also takes into account possible crosstalk in the M16 PMTs.

iii. Finding tracks - The algorithm [54] identifies small collections of hits in a single view that could serve as short track segments. These short track segments are combined to make longer segments. Longer segments from each view are combined 
to from a 3D track. The algorithm is tuned generously to find tracks. Therefore almost no tracks are missed. Muons constitute a majority of the tracks. If the track is fully contained inside the detector the energy deposited in each plane can be accumulated to find the energy of the muon. The resolution on the muon energy from range is $\sim 5 \%$.

iv. Finding the curvature of the tracks - For events that do not stop in the Detector the curvature of the track can be used to find the energy. To estimate curvature along the path a Kalman Filter is used [54]. This method takes into account the finite detector resolution and multiple Coulomb scattering as well as the effects of the magnetic field. The algorithm calculates the curvature at each point along the track. Due to the fact that $\nu_{\mu} \rightarrow \mu^{-}$and $\bar{\nu}_{\mu} \rightarrow \mu^{+}$, the sign of the curvature determines the initial neutrino type. The resolution on the muon energy from curvature is $\sim 10 \%$.

v. Finding showers - By looking for clusters of hits clustered in space and time hadronic showers can be formed. The total energy of each shower is proportional to the summed pulse height of all the hits.

vi. Forming neutrino events - This step combines information from the shower and track algorithms looking to combine them to form single neutrino interactions.

\subsection{Intensity Dependence of the Near Detector Data}

The number of neutrino interactions should depend linearly on the beam intensity. Deviations from linearity could indicate problems with the reconstruction software. Such a concern is warranted for the Near Detector data, where many many interactions per spill are seen. Figure 4.3 shows the mean number of events for $\nu_{\mu}$ and $\bar{\nu}_{\mu}$ as a function of beam intensity. The plots were made by counting the number events that pass the pre-selection cuts (see Section 6.1.1) for each spill. Care must be taken to include spills that did not 

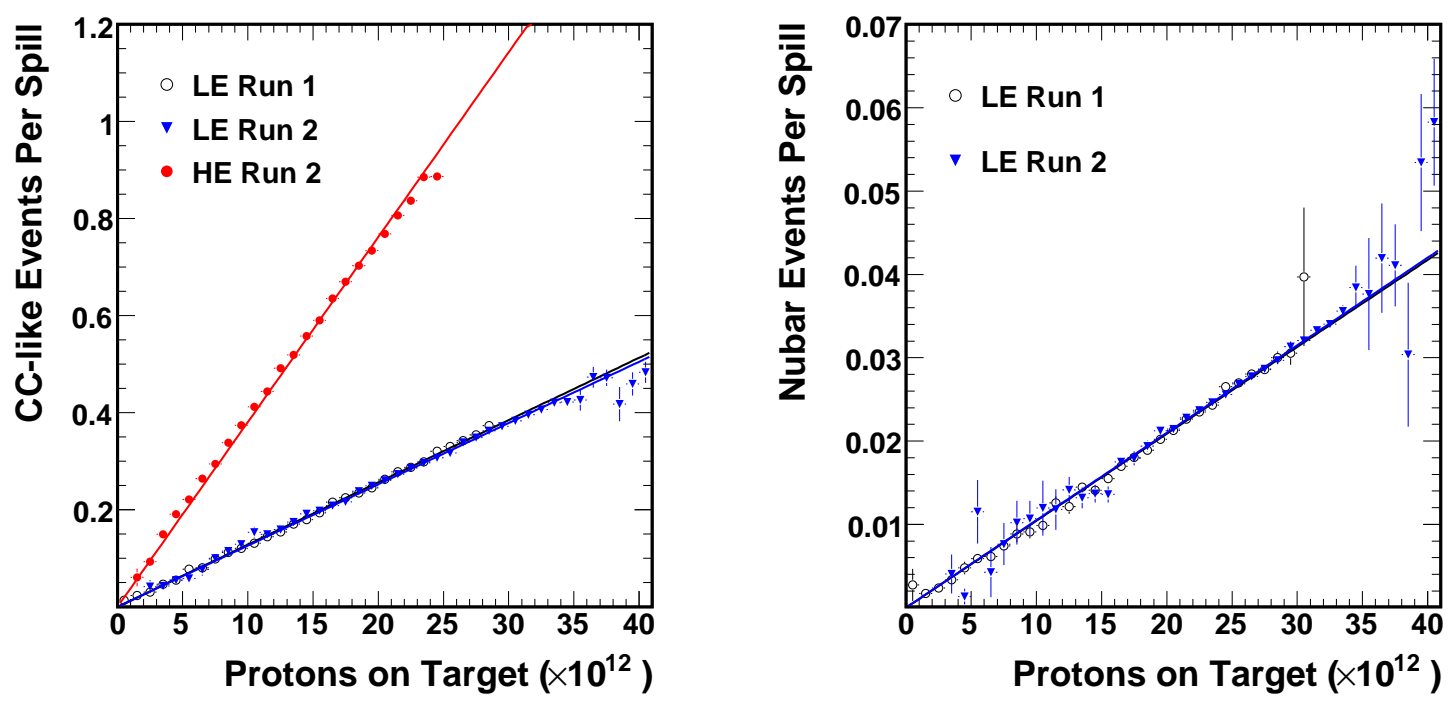

Figure 4.3: Left: The number of selected charged current events as a function of beam intensity for LE RunI (open circles) and RunII (blue triangles) and HE RunII (red circles). Right: The number of selected $\bar{\nu}_{\mu}$ events as a function of beam intensity for LE RunI (open circles) and RunII (blue triangles). Each data set is fit by a straight line constrained to pass through the origin.

produce any reconstructed neutrino events. Otherwise, the data will be skewed at low intensity. The data was also fit by a linear function constrained to pass through zero.

The data was seen to prefer a quadratic fit over a linear fit, showing a small loss in the number of events at higher intensity. This is consistent with studies from the Monte Carlo [55].

\subsection{Monte Carlo Simulation}

The simulation includes four independent stages: proton interactions in the target, propagation through the beamline, neutrino interactions in the MINOS detectors, and the detector response. Each successive step depends on the results from the previous step. Such modularity, speeds up processing time for the complete chain, and allows narrow focused studies on particular aspects. 


\subsubsection{Interactions in the Target}

The detailed interactions of the primary protons with the NuMI target are simulated using FLUKA 2005 [56]. FLUKA is a particle transport Monte Carlo capable of modeling hadronic interactions. FLUKA stores the outgoing particles from the target for reuse.

\subsubsection{Propagation Through the NuMI Beamline}

The next step uses the program GNUMI. GNUMI takes particles off the target and propagates them through the focusing horns to the decay pipe. The physics of the focusing by the horns and scattering off various components is modeled by GEANT3 [57]. The particles decay in the decay pipe, and the neutrino properties are stored in flux files. Because only a small fraction of the neutrinos produced actually intersect the detectors, the fluxes at the Near and Far Detectors are calculated by a reweighting scheme. Each event is given a weight based on the likelihood that it will be detected in the MINOS detectors. By weighting each neutrino event by this probability all of the neutrinos generated can be used in the flux calculation.

An additional weighting procedure preferentially tracks the higher energy hadrons produced from the target. A much greater number of low energy hadrons will be produced and the time involved in tracking all of these low energy hadrons is large. To enhance the statistics of the higher energy hadrons some of the low energy secondaries are selectively ignored and assigned an importance weight to correct for this procedure. This results in tracking about one out of two $15 \mathrm{GeV}$ hadrons and one in six $6 \mathrm{GeV}$ hadrons.

The flux files contain a list of neutrino parents along with their associated momentum and where they decayed. Using the reweighting schemes the flux at either detector, or anywhere else, can be calculated. 


\subsubsection{Neutrino Interactions in the MINOS Detectors}

GMINOS is responsible for randomly selecting neutrino events from the flux files for interaction. The neutrino path is sampled deciding whether or not to interact it in the detectors or the surrounding rock. The neutrino event generator, NEUGEN [58] (see the next section), models the neutrino-nucleon interactions. The NEUGEN results are passed to a GEANT3-based detector simulation (GMINOS) and propagated throughout the detector. GCALOR [59] models the hadronic shower development which determines the deposited energy in the scintillator strips.

\subsubsection{Modeling Neutrino Interactions with NEUGEN}

After specifying a neutrino and nucleus, NEUGEN simulates all of the neutrino interactions in MINOS detectors or rock. As discussed in Section 2.3, charged current events occur in QE, RES, or DIS interactions. The QE cross-section depends on the weak form factors of the nucleon, although effects (Fermi momentum, Pauli Blocking, etc) must also be taken into account. Nucleons are treated as bound in the nucleus based on the Fermi Gas model and hadrons are allowed to reinteract in the nucleus. RES events are modeled in NEUGEN using a Rein-Sehgal [60] model. Both RES and QE events are parametrized in NEUGEN using a dipole approximation for the weak axial form factor. Figure 4.4 shows the $\nu_{\mu}$ charged current cross-section as a function of neutrino energy as used in NEUGEN.

\subsubsection{Light Transport}

Once the energy from individual strips have been recorded the final stage is to incorporate the simulation of the detectors. Light from the scintillator is transported through the wavelength shifting fiber to the PMTs and digitized into individual hits. At this point, the hits can use same reconstruction code as the real data. 


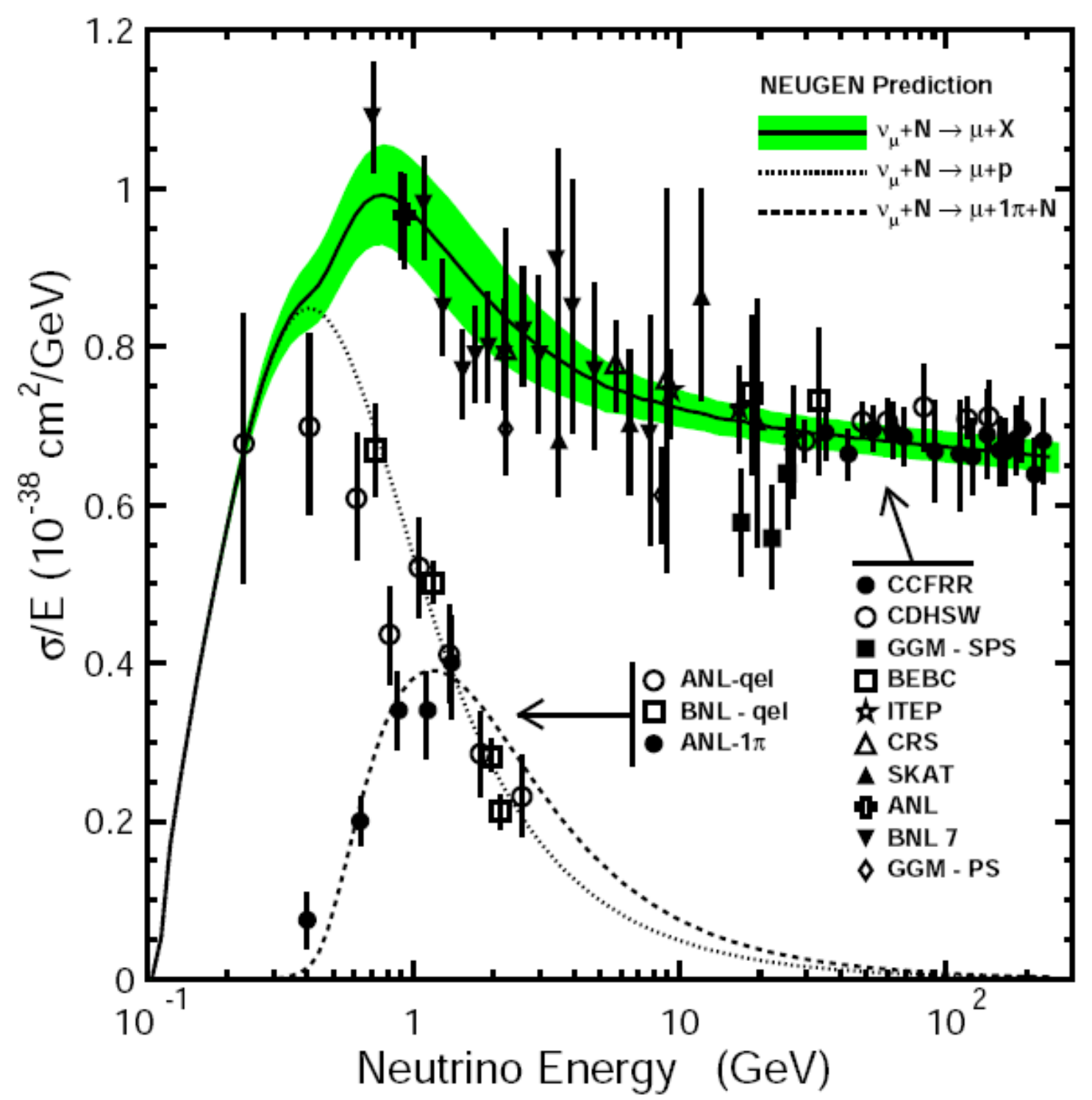

Figure 4.4: The charged current neutrino cross-section as a function of neutrino energy for inclusive scattering, quasi-elastic, and single pion production. This calculation is compared to experimental data. The green band indicates the theoretical uncertainty. Taken from [49]. 


\section{Chapter 5}

\section{Hadron Production Tuning}

The number of neutrinos detected is a function of the neutrino flux, the cross-section and the total amount of detector material. A good understanding of the neutrino flux is important for a precision measurement of oscillation parameters. The largest uncertainty on the flux comes from hadron production.

The calculation of the number of hadrons produced from interactions in the target has plagued many neutrino experiments. In neutrino experiments, hadrons serve as intermediary states to the neutrinos and are not directly detected. Few experiments have been dedicated to making these measurements. A knowledge of the hadron yield as a function of momenta and angle is essential to predicting the neutrino energy spectrum. Several experiments have used neutrino data to constrain the hadron production [61, $62,63,64]$. One of the unique features MINOS offers, is the ability to sample different regions of momentum space, and use it to constrain the underlying physics of hadron production. Section 3.1.5 showed how different beam configurations sample different regions of momenta space.

Knowing the hadron production is particularly important at high energies where hadrons travel through the horns unfocused. Other uncertainties that don't impact the flux prediction as much come from the cross-sections, detector modeling and background estimation. Since these uncertainties have different energy dependencies, the data in the 
Near Detector can be used to constrain them. For example, errors in the knowledge of cross-sections at a given energy will appear in all beams as a deviation from the Monte Carlo simulations. However, the energy dependence due to errors in the beam modeling will vary for different configurations allowing for a decoupling of these errors from other sources.

The effect on the energy spectrum from different uncertainties can be calculated from the Monte Carlo. If these uncertainties can be characterized by a relatively small number of parameters, they can be implemented in a fit of the Monte Carlo to the large amount of data in the Near Detector. This chapter will describe the parameterization of the important uncertainties and the details of the fit to the Near Detector data. This fit will be able to reduce the systematic error from hadron production and give a more accurate prediction of the flux. The corrections to the Monte Carlo simulations derived from the fit in the Near Detector can also be applied to the Monte Carlo in the Far Detector to make a prediction of the unoscillated neutrino spectrum (see Section 6.2).

The approach described in this chapter is an extension of the one pioneered by Zarko Pavlovich [45] for the analysis of an earlier data set. The implementation is original to this thesis. It also forms the basis for the work and plots presented in published MINOS results of the same data set described here [48].

\subsection{Existing Hadron Production Data}

To date, relatively few measurements of hadron production from high-energy protons interactions have been made. The most relevant to MINOS are measurements made by Atherton et al. [65], SPY [66] and Barton et al. [67]. Figure 5.1 shows these data sets overlaid with the probability that a $\pi^{+}$will produce a neutrino interaction in the Near Detector (see Figure 3.6). While the data does cover some of the region of the LE beam, there is no data for very low $p_{T}$ or for the region important to the HE beam. 


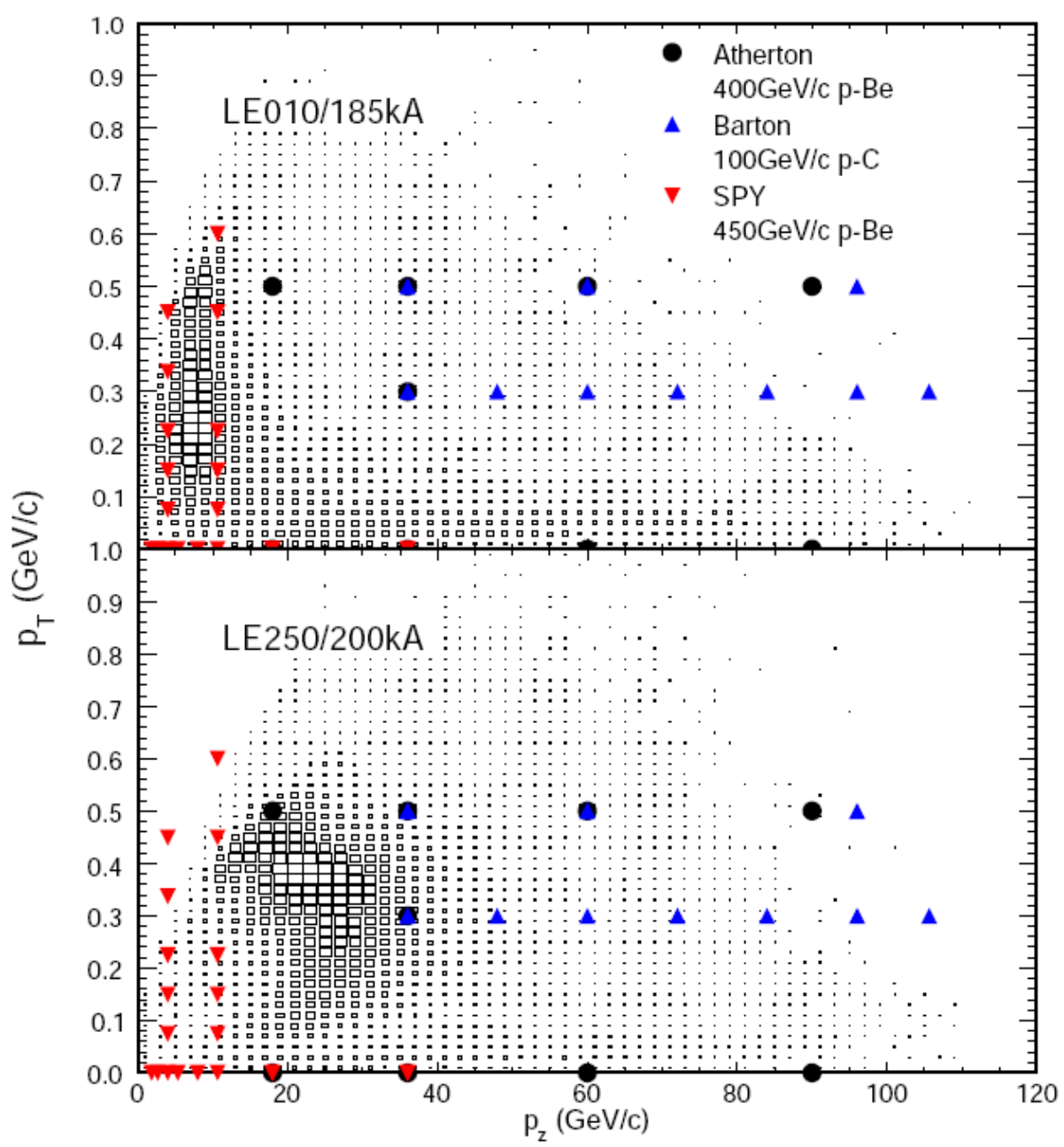

Figure 5.1: The boxes represent the probability that a $\pi^{+}$will interact in the Near Detector and the points indicate where hadron production experiments have made measurements The red, black and blue points come from Atherton [65], Spy [66] and Barton [67] respectively. Top: the le010z185i beam configuration. Bottom: the le250z200i configuration. Figure taken from [45]. 
Even these measurements cannot necessarily be applied to MINOS. Complications arise because the data was taken in different environments. Some of these complications include:

i. Most data has been taken by single arm spectrometers sensitive to only selected points of $p_{T}$ and $p_{Z}$ space requiring interpolation.

ii. Experiments have taken data at different beam momenta.

iii. Experiments have used targets of different lengths and materials.

All of these factors must be extrapolated to the NuMI beam conditions before they can be used. Because of these difficulties and the overall lack of data, estimates of the uncertainties have been difficult to make. Figure 5.2 compares four different models of the neutrino flux at the Near Detector from MARS [68], GEANT-FLUKA, BMPT [69], and Malensek [70]. The range of prediction varies from 10-25\% as function of neutrino energy.

The MIPP [71] experiment was specifically designed to address the question of hadron production for NuMI. MIPP is a full acceptance spectrometer with excellent particle identification. Using a spare NuMI target MIPP has successfully taken data, but is still in the process of analyzing the results. Preliminary studies have been done [72], but the full use of the MIPP data in constraining the hadron production data for MINOS will await the official release of their data. Comparisons with the preliminary MIPP results are shown in Section 5.6.3

\subsection{Hadron Production Parameterization}

Feynman [73] proposed that the energy of secondary hadrons should show some degree of scaling with beam momentum. He used the scaling variable $x_{F}=2 p_{L} / \sqrt{s}$, where $p_{L}$ and $\sqrt{s}$ are the longitudinal momentum and total energy in the center of momentum frame. One result of this is that the shape of the $p_{T}$ distributions should be similar for different 

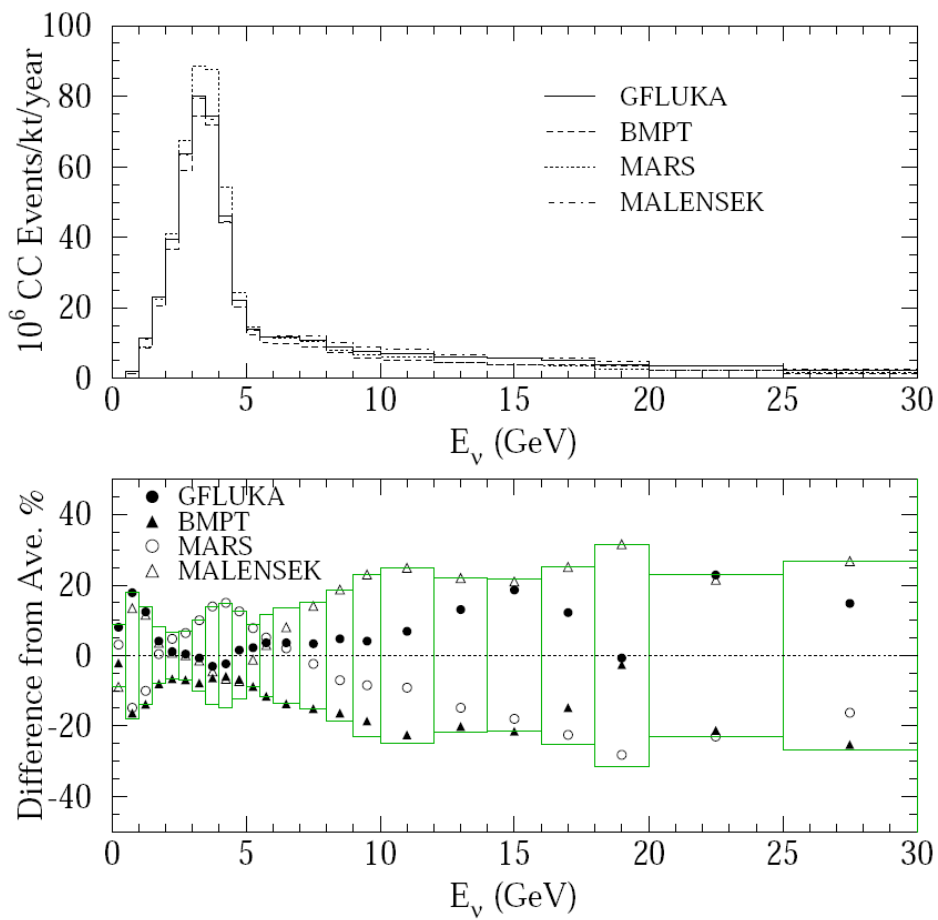

Figure 5.2: Comparison of different models used to predict the neutrino flux. Top: the Near Detector flux. Bottom: ratio of each model to the average. Taken from [45]. 
incident beam momenta. For the purposes of MINOS a sufficiently good scaling variable is $x_{l a b}=p_{L} / p_{0}$, where $p_{L}$ is the longitudinal momentum of the hadron in the lab and $p_{0}$ is the momentum of the primary particle. It can be shown that for $p_{0}>>M_{p}$ and $p_{L}>m_{\pi}, x_{l a b} \approx x_{F}$. The variables $x_{l a b}$ and $x_{F}$ will be used interchangeably in this chapter. The hadron production parameterization was developed by Zarko Pavlovich [45] and will be described here for completeness. For our studies we employ a parameterization of hadron production using the following functional form:

$$
\frac{d^{2} N}{d p_{T} d x_{F}}=\left[A+B * p_{T}\right] * \exp \left(-C p_{T}^{3 / 2}\right)
$$

where $A, B$, and $C$ are parameters that modify the hadron production and $x_{F}=$ $p_{Z} / p_{\text {proton }}$. The parameter $A$ determines the yield at low $p_{T}, B$ determines the rising edge, and $C$ determines how fast the function falls off at high $p_{T}$.

Values of $A, B$, and $C$ can be found as function of $x_{F}$ by fitting the $p_{T}$ distributions to Equation 5.1 in distinct bins of $x_{F}$. Figure 5.3 shows the hadron production yields as calculated by FLUKA for different $x_{F}$ regions with the corresponding fit to equation 5.1, separately for kaons and pions. As can be seen there is some disagreement, but exact agreement is not essential for our purposes, only the ability to provide adequate knobs that change the essential features of the shape of the hadron spectra.

The results from the fits to $A, B$, and $C$ can be further represented as parametric functions of $x_{F}$. The parametric functions take the form:

$$
\begin{aligned}
A\left(x_{F}\right) & =a_{1} *\left(1-x_{F}\right)^{a_{2}} *\left(1+a_{3} * x_{F}\right) * x_{F}^{-a_{4}} \\
B\left(x_{F}\right) & =b_{1} *\left(1-x_{F}\right)^{b_{2}} *\left(1+b_{3} * x_{F}\right) * x_{F}^{-b_{4}} \\
C\left(x_{F}\right) & =c_{1} / x_{F}^{c_{2}}+c_{3}\left(\text { for } x_{F}<0.22\right) \\
& =c_{1} * \exp \left(c 2\left(x_{F}-c_{3}\right)+c 4 x_{F}+c_{5} \cdot\left(\text { for } x_{F}>0.22\right) .\right.
\end{aligned}
$$




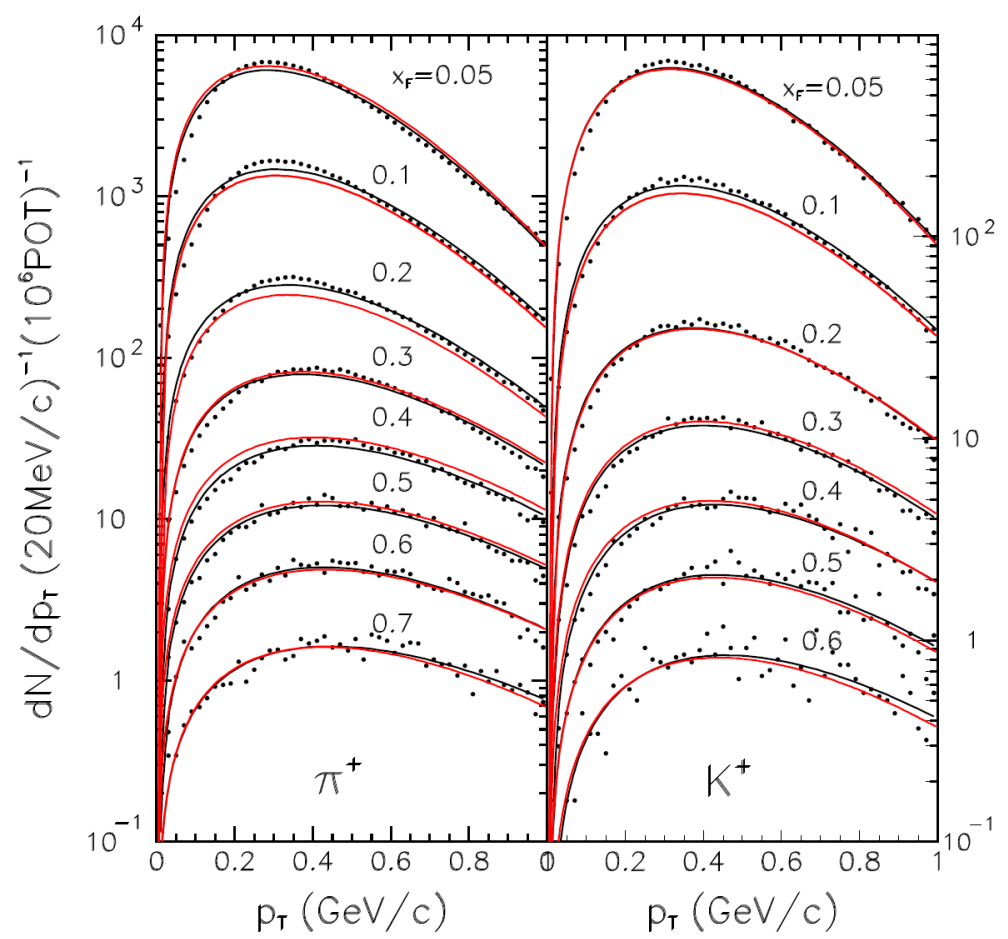

Figure 5.3: Monte Carlo data from FLUKA on the production of $\pi^{+}$(left) and $K^{+}$(right) in different slices of $x_{F}$. The solid lines indicate fits to the data using Equation 5.1. Taken from $[45]$.

These are loosely based on the BMPT [69] parameterization. The results of the parameter fits shown in Figure 5.4 provide values for $A, B$, and $C$ for any value of $x_{F}$.

\subsection{Constraining the Monte Carlo: The Fit to the Near Detector Data}

A fit is done with all of the data from the Near Detector data and parameters that can be varied so as to minimize the differences between the data and Monte Carlo. The parameters can be divided roughly into three different sections: beam, hadron production, and detector. The beam parameters focus on alignment and focusing effects of the NuMI beam. The hadron production parameters describe how $A, B$ and $C$ from the previous 

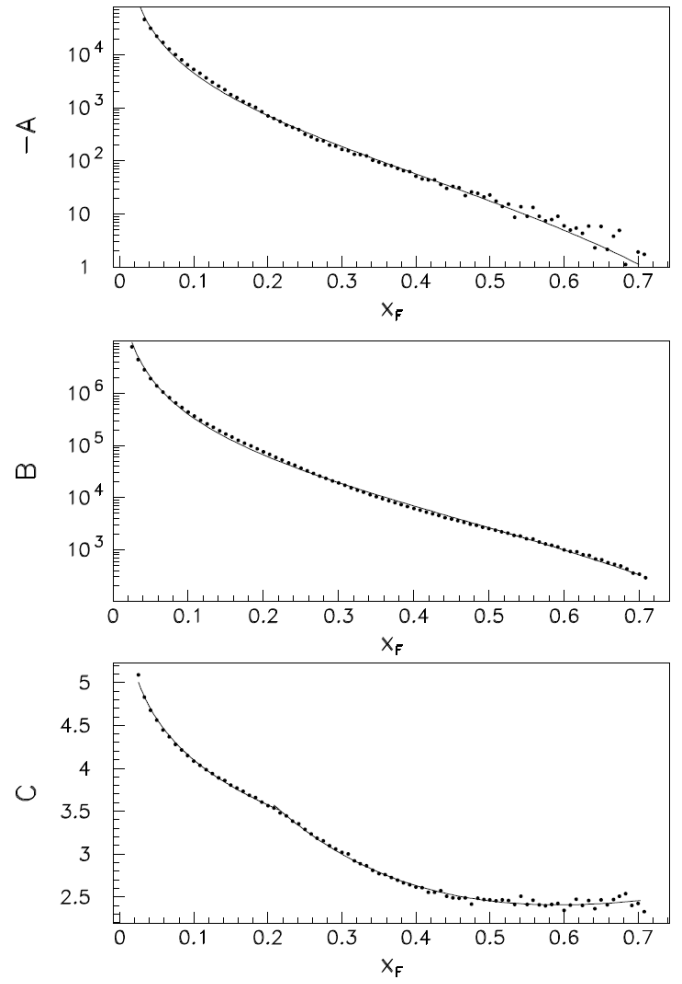

Figure 5.4: The coefficients for $A, B$, and $C$ as a function of $x_{F}$. The fit of Equation 5.3 is the solid line. Taken from [45]. 
section are modified to change the shape of the underlying production yield. The detector parameters encompass cross-sections, energy scales and the NC background.

The minimization routine of the fit is provided by MINUIT [74] interfaced with the ROOT [75] package. The fit uses samples separated as $\nu_{\mu}$ and $\bar{\nu}_{\mu}$ events from 10 different beam configurations. The data and Monte Carlo spectra are stored in histograms and the $\chi^{2}$ function that is minimized is defined as:

$$
\chi^{2}=\sum^{\text {beam }} \sum^{\nu \text { type bins }} \frac{\left(N_{\text {data }}-N_{\mathrm{MC}}(\alpha)\right)^{2}}{\sigma_{\text {data }}^{2}+\sigma_{\mathrm{MC}}^{2}}+\sum_{j}^{\text {parameters }}\left(\frac{\Delta \alpha_{j}}{\sigma_{j}}\right)^{2},
$$

where the sum is over all beam configurations, $\nu_{\mu}$ and $\bar{\nu}_{\mu}$ events and over all the bins of the histograms, $N_{\text {data }}$ and $N_{\mathrm{MC}}$ are the histogram bin contents for data and Monte Carlo, $\sigma_{\text {data }}$ and $\sigma_{\mathrm{MC}}$ are the statistical uncertainties on the bin contents, $\Delta \alpha_{j}$ is the difference between the parameter value and the nominal value used in the Monte Carlo simulation and $\sigma_{j}$ is the uncertainty. Additional constraints are provided as detailed in the following sections.

\subsubsection{Hadron Production Parameters}

To describe the hadron production, 16 parameters are introduced: 6 for $\pi^{+}$and $K^{-}$and 2 for $\pi^{-}$and $K^{-}$. Because the sensitivity in momentum space is larger for positive mesons, more parameters are used. The six parameters for the positive mesons are defined as follows for $\pi^{+}$:

$$
\begin{aligned}
& A^{\prime}\left(x_{F}\right)=\left(\operatorname{par} 0+\operatorname{par} 1 \cdot x_{F}\right) \cdot A\left(x_{F}\right) \\
& B^{\prime}\left(x_{F}\right)=\left(\operatorname{par} 2+\operatorname{par} 3 \cdot x_{F}\right) \cdot B\left(x_{F}\right) \\
& C^{\prime}\left(x_{F}\right)=\left(\operatorname{par} 3+\operatorname{par} 5 \cdot x_{F}\right) \cdot C\left(x_{F}\right),
\end{aligned}
$$


and for $K^{+}$:

$$
\begin{aligned}
& A^{\prime}\left(x_{F}\right)=\left(\operatorname{par} 6+\operatorname{par} 7 \cdot x_{F}\right) \cdot A\left(x_{F}\right) \\
& B^{\prime}\left(x_{F}\right)=\left(\operatorname{par} 8+\operatorname{par} 9 \cdot x_{F}\right) \cdot B\left(x_{F}\right) \\
& C^{\prime}\left(x_{F}\right)=\left(\operatorname{par} 10+\operatorname{par} 11 \cdot x_{F}\right) \cdot C\left(x_{F}\right) .
\end{aligned}
$$

Each individual hadron is given a weight by the fit based on these parameters:

$$
\operatorname{Weight}\left(\pi^{+}, K^{+}\right)=\frac{\left[A^{\prime}+B^{\prime} p_{T}\right] * \exp \left(-C^{\prime} p_{T}^{3 / 2}\right)}{\left[A+B p_{T}\right] * \exp \left(-C p_{T}^{3 / 2}\right)}
$$

Because the weights are calculated through this ratio, the method does not critically depend on the how well the parameterization is able to match the data. The weights for the negative mesons are defined as follows:

$$
\begin{aligned}
\operatorname{Weight}\left(\pi^{-}\right) & =\left(\operatorname{par} 12+\operatorname{par} 13 \cdot x_{F}\right) * \operatorname{Weight}\left(\pi^{+}\right) \\
\operatorname{Weight}\left(K^{-}\right) & =\left(\operatorname{par} 14+\operatorname{par} 15 \cdot x_{F}\right) * \operatorname{Weight}\left(K^{+}\right)
\end{aligned}
$$

By using the same initial weights, the ratios $\pi^{+} / \pi^{-}$and $K^{ \pm} / \pi^{ \pm}$are preserved to first order.

The $\nu_{\mu}$ beam does not have sensitivity to neutrinos from $K_{L}^{0}$, but they do contribute to the $\nu_{e}$ component of the beam. We follow the prescription from [45] to relate the neutral kaon component to the charged kaons. Assuming isospin symmetry, $u_{v} / d_{v}=2$, $u_{s}=\bar{u}_{s}=d_{s}=\bar{d}_{s}$ and $s_{s}=\bar{s}_{s}$ where the subscript $v$ is for valence quarks and $s$ for sea quarks. The number of $K_{L}^{0}$ is related to $N\left(K^{+}\right)$and $N\left(K^{-}\right)$by

$$
N\left(K_{S}^{0}\right)=N\left(K_{L}^{0}\right)=\frac{N\left(K^{+}\right)+3 N\left(K^{-}\right)}{4}
$$

This simple model agrees with direct measurements of $K_{S}$ to within $15 \%$ up to $x_{F}=$ $0.5[69]$. 
Because very different values for these parameters can give similar results the hadron production parameters are not constrained in the fit. The value of weights are restricted to be within the range of \pm 10 to avoid numerical problems in the fit. Constraints on the hadron production come from penalty terms introduced for the particle production ratios and the shift in the average $p_{T}$.

The data from the NA49 hadron production experiment is used to constrain the $\pi^{+} / \pi^{-}$ratio. We assumed a $5 \%$ uncertainty which is larger than the errors given in [76]. One concern in using this data is that it was taken with a much thinner target. The MINOS target is $94 \mathrm{~cm}$ in length while the NA49 target was only $7 \mathrm{~cm}$ long. To address the possible impact of thin/thick target, a Monte Carlo simulation was run using FLUKA to look at the resulting $\pi^{+} / \pi^{-}$when only the first NuMI target segment is used. This corresponds to a target $2 \mathrm{~cm}$ long and 0.04 interaction lengths. The differences between this simulation and the full target were within the $5 \%$ uncertainty assumed for the NA49 data. The other set of hadron production constraints are from FLUKA:

i. $\pi^{+} 15 \mathrm{MeV}$ shift for the mean $p_{T}$

ii. $K^{+} 15 \mathrm{MeV}$ shift for the mean $p_{T}$

iii. $20 \%$ constraint on $K^{+} / K^{-}, K^{+} / \pi^{+}$, and $K^{-} / \pi^{-}$

The spread of $<p_{T}>$ predicted by different models motivates the $15 \mathrm{MeV}$ penalty terms and the $20 \%$ peanlty terms on production ratios.

\subsubsection{Beam Parameters}

A comparison of the neutrino energy spectrum from RunI and RunII for the LE beam configuration shows a difference of about $5 \%$ at the peak of the energy spectrum at 5 $\mathrm{GeV}$. The most likely explanation for such a shift is that the target position was different from RunI relative to RunII because a new target was installed at the beginning of RunII. This fact questioned the reliability of the target position for all the beam configurations. Fits where all the target positions were allowed to float were able to produce better 
agreement with the data. For these reasons the target position was introduced as a parameter in the fit for all the beam configurations. This adds 6 parameters for the different configurations; three in RunI for the le010z, le100z, and le250z positions and and three in RunII for the le010z, le150z, and le250z positions. An uncertainty of $\pm 1 \mathrm{~cm}$ from the nominal position was used in the corresponding penalty terms. There is no way to observationally confirm the position of the target for previous configurations therefore the difference between a fit with and without the target positions floating will be added to the systematic uncertainty.

\subsubsection{Detector Parameters}

Seven parameters are considered in the fit for neutrino interaction modeling:

i. $M_{A}$ for quasi-electric scattering $(1 \sigma=15 \%)$ (see Section 2.3 )

ii. $M_{A}$ for resonance scattering $(1 \sigma=15 \%)$

iii. $\bar{\nu}_{\mu}$ charged current cross-section ( $1 \sigma=$ up to $\left.30 \%\right)$

iv. NC scale for $\nu_{\mu}$-like $(1 \sigma=30 \%)$

v. $\mathrm{NC}$ scale for $\bar{\nu}_{\mu}$-like $(1 \sigma=30 \%)$

Figure 5.5 shows the effect of scaling $M_{A}$ for quasi-elastic and resonance events. Parameters for DIS events were not included in the fit because of strong correlations with hadron production parameters. The cross-section are also better known for DIS events than quasi-elastic and resonance events.

An energy dependent scale factor for the $\bar{\nu}_{\mu}$ cross-section is used that varies smoothly from 0 to $25 \mathrm{GeV}$ and is parameterized by:

$$
\text { ScaleFactor }=a+\frac{2 *(1-a) * E_{\text {True }}}{b}+\frac{(a-1) * E_{\text {True }}^{2}}{b^{2}},
$$

where $E_{\text {True }}$ is the true neutrino energy, $a$ is a parameter for the fit, and $b$ is fixed at $25 \mathrm{GeV}$. No weighting is done for events with true energy greater than $25 \mathrm{GeV}$. Figure 5.6 


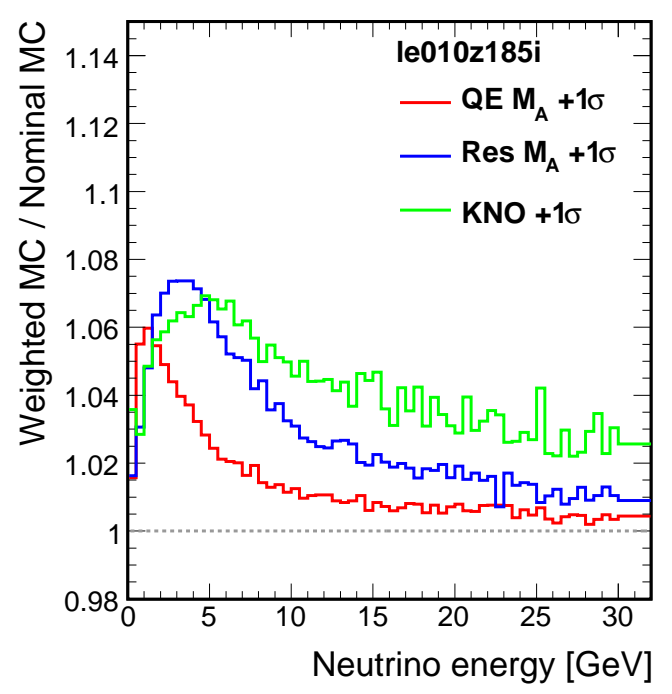

Figure 5.5: The effect of changing the QE and RES $M_{A}$ by one $1 \sigma$, where in this case $1 \sigma=15 \%$ and also shifting the KNO parameters by $1 \sigma$.

shows the function for a value of $a=0.95$. An additional constraint to the $\bar{\nu}_{\mu}$ cross-section is included that is $30 \%$ at low energy, and slowly approaches 0 at $25 \mathrm{GeV}$.

Three parameters are considered to account for the uncertainty in the neutrino energy reconstruction:

i. Shower energy offset $(1 \sigma=50 \mathrm{MeV})$,

ii. Shower energy scale ( $1 \sigma=$ energy dependent function),

iii. Muon energy scale $(1 \sigma=4 \%)$.

The following functional form is used to describe the uncertainty on the shower energy scale:

$$
\begin{aligned}
\epsilon_{1} & =0.04 \\
\epsilon_{2} & =0.1-0.02 * E_{\text {shw }}+0.001 * E_{\text {shw }}^{2}\left(\text { for } E_{\text {shw }}<10 \mathrm{GeV}\right) \\
& =0\left(\text { for } E_{\text {shw }}>10 \mathrm{GeV}\right) \\
\epsilon_{\text {cal }} & =0.057 \\
\epsilon_{\text {total }} & =\sqrt{\epsilon_{1}^{2}+\epsilon_{2}^{2}+\epsilon_{\text {cal }}^{2}}
\end{aligned}
$$




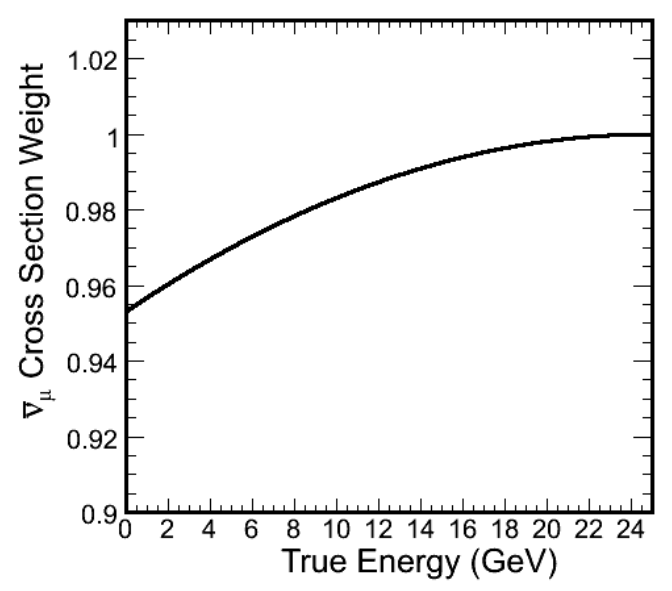

Figure 5.6: The weighting given for the $\bar{\nu}_{\mu}$ cross-section uncertainty. In this plot the value of the parameter was set to 0.95 .

where $\epsilon_{1}$ and $\epsilon_{2}$ account for uncertainties in the hadronization model and nuclear effects as a function of true shower energy, and $\epsilon_{c a l}$ is from the absolute energy calibration from the Calibration Detector (see Section 3.2.3). The total uncertainty $\epsilon_{\text {total }}$ is calculating by adding the different components in quadrature.

\subsection{Data Sets}

A set of pre-selection cuts was applied (see Section 6.1.1) to select CC-like neutrino events.

A majority of the MINOS data (see Table 4.1) is taken in the le010z185i beam configuration since it is most sensitive to neutrino oscillations. However, in order not to be completely dominated by the data in this configuration but rather be sensitive to other beam configurations, only a subset of the data from the le010z185i and le250z200i beams is used. Complete runs were selected from the entire data set until the desired number of protons on target (POT) was reached. As a check, a comparison was done between the subsample used in the fit and the data sets from which they were selected. Figure 5.7 compares the reconstructed neutrino, muon and shower energy. These plots shows that the differences between all the data and the subsample was within a few percent. 

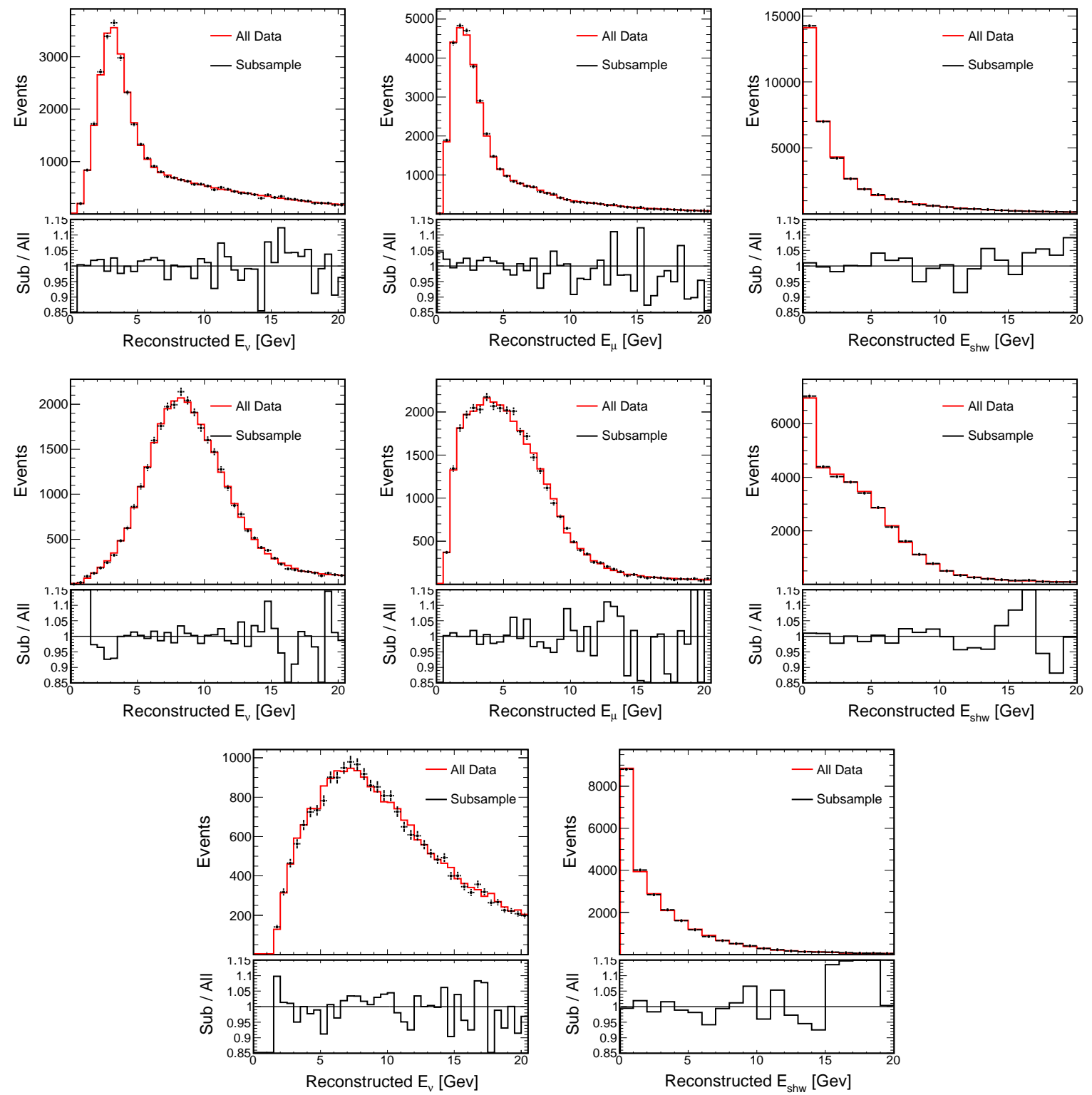

Figure 5.7: Top Row: A comparison of the subsample used in the fit to the total data for the neutrino (left), muon (middle) and shower (right) energies. This shows data from the $\nu_{\mu}$ le010z185i beam. Middle Row: A comparison of the subsample used in the fit to the total data for the neutrino (left), muon (middle) and shower (right) energies. This shows data from the $\nu_{\mu}$ le250z200i beam. Bottom Row: A comparison of the subsample used in the fit to the total data for the neutrino (left), and shower (right) energies. This shows data from the $\bar{\nu}_{\mu}$ le010z185i beam. 


\begin{tabular}{lccc}
\hline \hline Beam Configuration & Data POT $\left(\times 10^{18}\right)$ & MC POT $\left(\times 10^{18}\right)$ & Variables in Fit \\
\hline le010z000i RunI & 2.59 & 6.00 & enu \\
le010z000i RunII & 2.75 & 6.00 & enu \\
le010z170i & 1.44 & 3.54 & enu \\
le010z185i RunI & 3.26 & 17.10 & enu, emu, eshw \\
le010z185i RunII & 3.03 & 17.10 & enu, emu, eshw \\
le010z200i & 1.35 & 3.58 & enu \\
le100z200i & 0.51 & 3.51 & enu, emu, eshw \\
le150z200i & 0.84 & 3.49 & enu, emu, eshw \\
le250z200i RunI & 0.78 & 3.45 & enu, emu, eshw \\
le250z200i RunII & 0.87 & 3.45 & enu, emu, eshw \\
\hline \hline
\end{tabular}

Table 5.1: Amount of data and Monte Carlo used for the $\nu_{\mu}$ sample. The column "Variables in the fit" indicates which information was used in the fit for that particular beam: "enu" is the neutrino energy, "emu" is the muon energy, and "eshw" is the shower energy.

The reconstructed neutrino energy is taken to be the sum of the individual shower and muon energies. Tables 5.1 and 5.2 show the amount of each data that is used for $\nu_{\mu}$ and $\bar{\nu}_{\mu}$ samples. The fit is done with three individual histograms of muon, shower, and neutrino energy except where noted.

\subsection{Smearing}

A majority of the parameters defined for the fit scale the histogram bin contents up or down. In contrast, the energy scale parameters shift events between bins. This shifting will cause, depending on the amount of Monte Carlo used, discontinuities in the $\chi^{2}$ surface. This causes problems for the fit. The MIGRAD minimization method used in MINUIT relies on the second derivatives with respect to the parameters and cannot handle discontinuous $\chi^{2}$ surfaces. Other minimization algorithms such as the SIMPLEX method are immune to such problems, but cannot give an estimate on the uncertainty of individual fit parameters and take more time. 


\begin{tabular}{lccc}
\hline \hline Beam Configuration & Data POT $\left(\times 10^{18}\right)$ & MC POT $\left(\times 10^{18}\right)$ & Variables in Fit \\
\hline le010z000i RunI & 2.59 & 6.00 & enu \\
le010z000i RunII & 2.75 & 6.00 & enu \\
le010z170i & 1.44 & 3.54 & enu \\
le010z185i RunI & 26.92 & 17.10 & enu \\
le010z185i RunII & 28.79 & 17.10 & enu \\
le010z200i & 1.35 & 3.58 & enu \\
le100z200i & 1.02 & 3.51 & enu \\
le150z200i & 1.67 & 3.49 & enu \\
le250z200i RunI & 1.55 & 3.45 & enu \\
le250z200i RunII & 14.43 & 3.45 & enu \\
\hline \hline
\end{tabular}

Table 5.2: Amount of data and Monte Carlo used for the $\bar{\nu}_{\mu}$ sample. All $\bar{\nu}_{\mu}$ samples only use the neutrino energy.

One way to smooth out the $\chi^{2}$ surface is to smear each event and allow events near bin boundaries to contribute to neighboring bins. This can remove the discontinuities in the $\chi^{2}$ surface and allow MINUIT to correctly get errors on the parameters. The biweight kernel was chosen to perform the smearing. This function is defined as $\left(1-x^{2}\right)$ from $x=-1$ to 1 . The width of the function is normalized to the desired smearing width. A smearing of $1 \%$ of the neutrino, muon and shower energy was chosen and is well below the resolution of the detector.

Figure 5.8 shows the effect for the shower energy scale with and without smearing for different widths. The fit was run with several different widths and the results were found to be independent of the widths chosen. For consistency, the smearing was applied to both data and Monte Carlo.

\section{$5.6 \quad$ Fit Results}

Figures 5.9 through 5.14 show the results of the fitting procedure for each of the 10 different beam configurations. Each plot shows four separate histograms: the Near Detector data, the nominal Monte Carlo, the results of the full fit and a fit where the target positions were kept fixed. Results are shown for the $\nu_{\mu}$, neutrino, muon and shower energy 


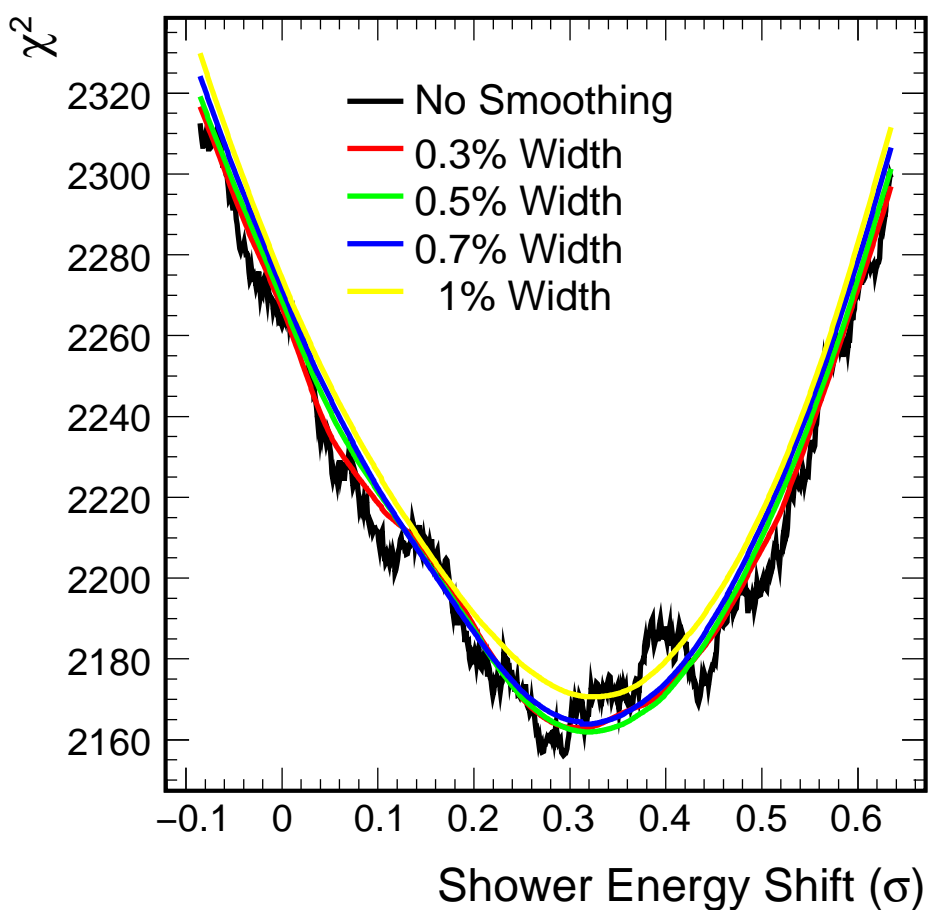

Figure 5.8: The $\chi^{2}$ surface as a function of the shower energy scale for different smearing widths. 
distributions and the $\bar{\nu}_{\mu}$ antineutrino energy. Table 5.3 summarizes the contribution of the $\chi^{2}$ from each beam configuration and the number of bins. Tables 5.4, 5.5 and 5.6 list the values of the best fit parameters. 

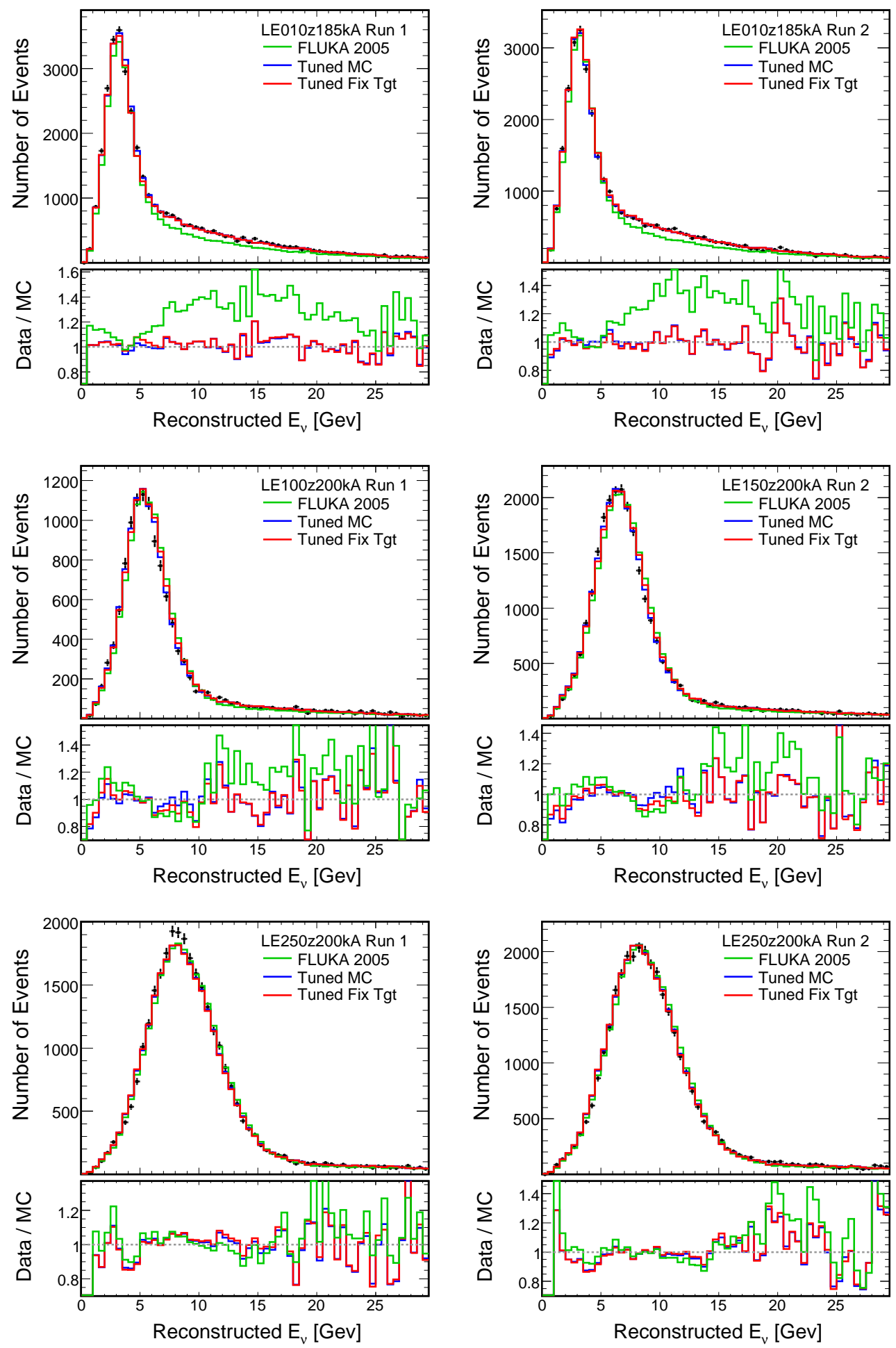

Figure 5.9: Neutrino energy spectrum for the $\nu_{\mu}$ charged current sample in the Near Detector. Black points are the data, Red lines are the untuned Monte Carlo based on FLUKA, the blue is the result after the fit and purple shows a fit with the target position fixed. 

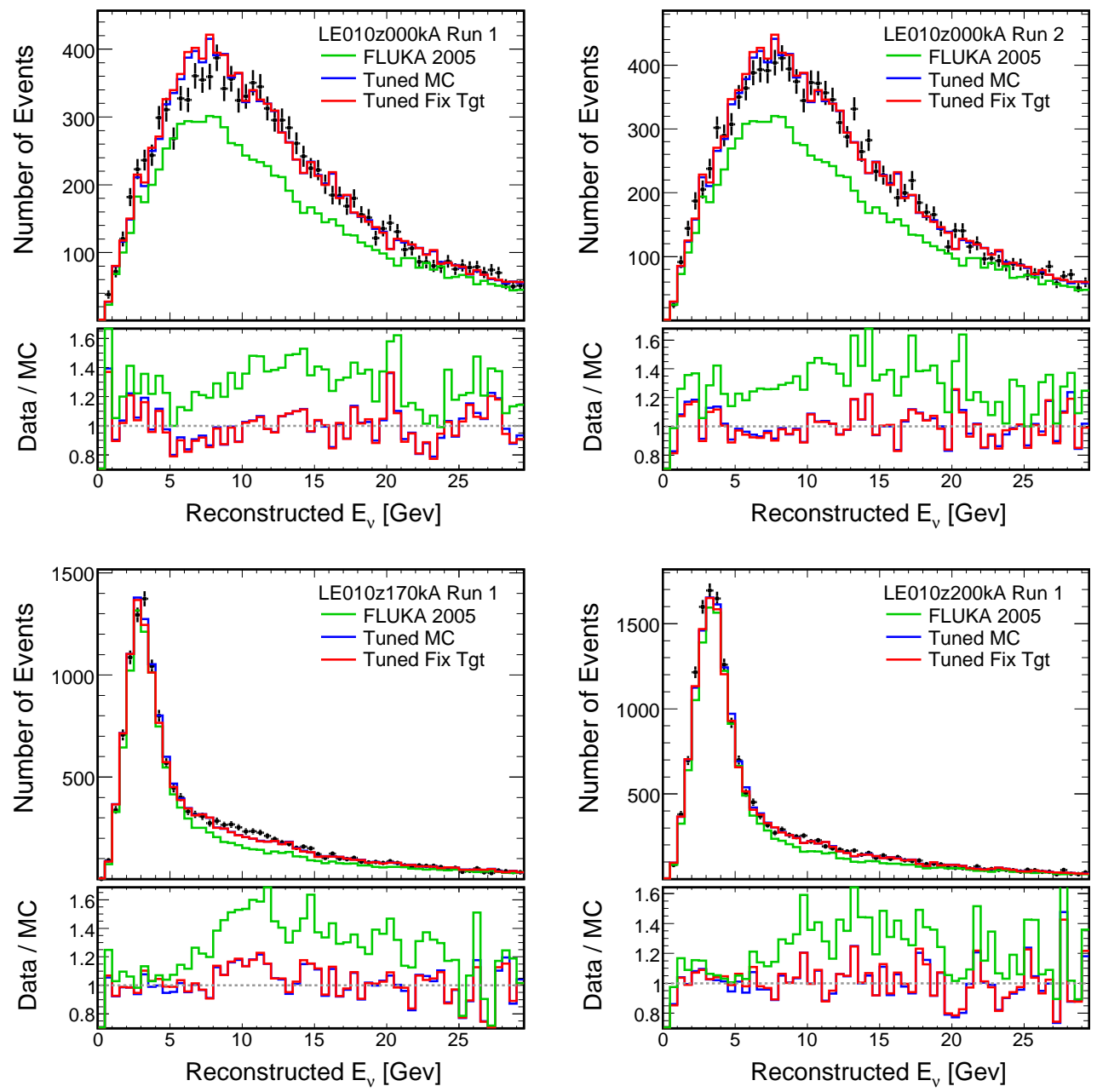

Figure 5.10: Neutrino energy spectrum for the $\nu_{\mu}$ charged current sample in the Near Detector. Black points are the data, green lines are the untuned Monte Carlo based on FLUKA, the blue is the result after the fit and red shows a fit with the target position fixed. 

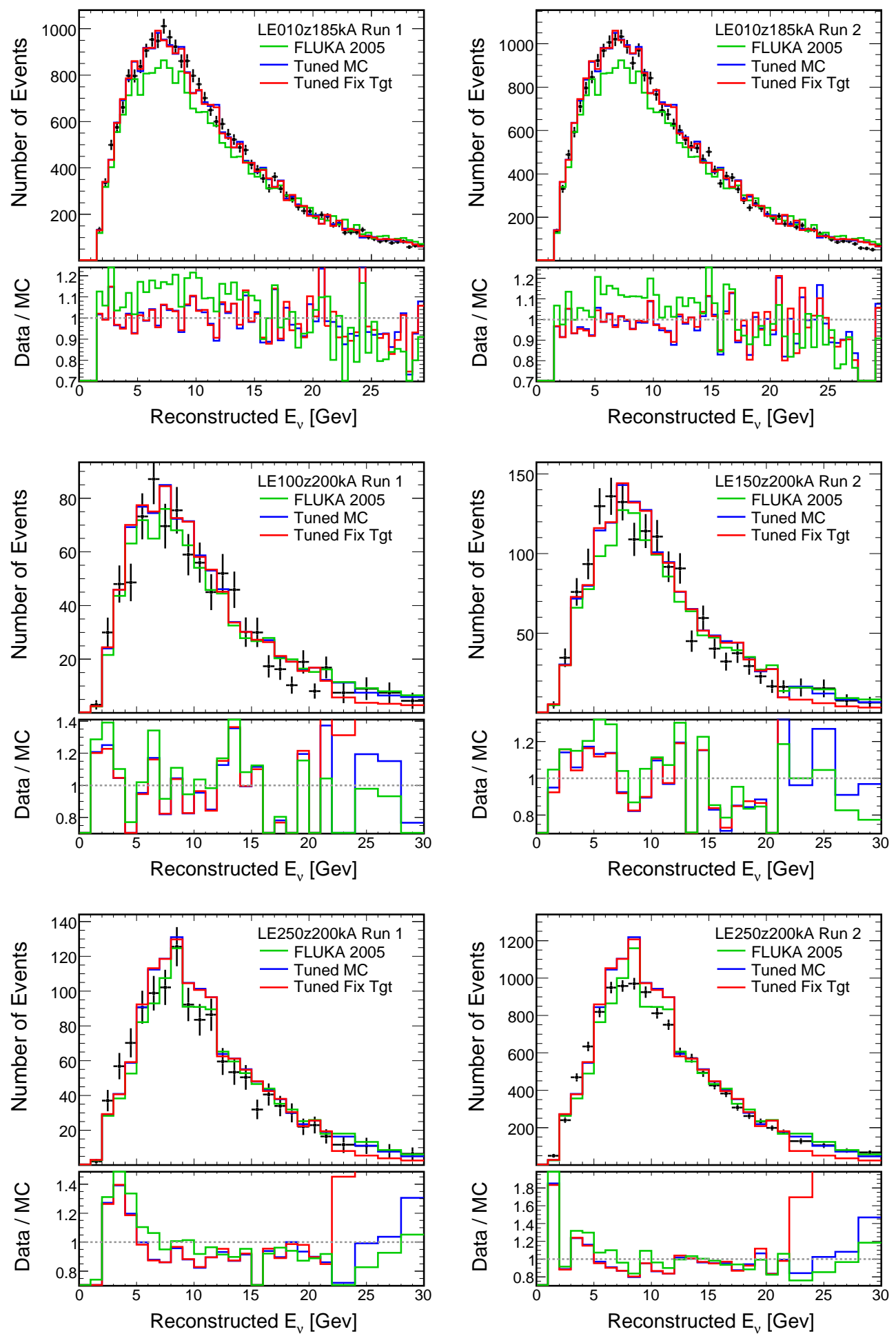

Figure 5.11: Anti-neutrino energy spectrum for the $\bar{\nu}_{\mu}$ charged current sample in the Near Detector. Black points are the data, green lines are the untuned Monte Carlo based on FLUKA, the blue is the result after the fit and red shows a fit with the target position fixed. 

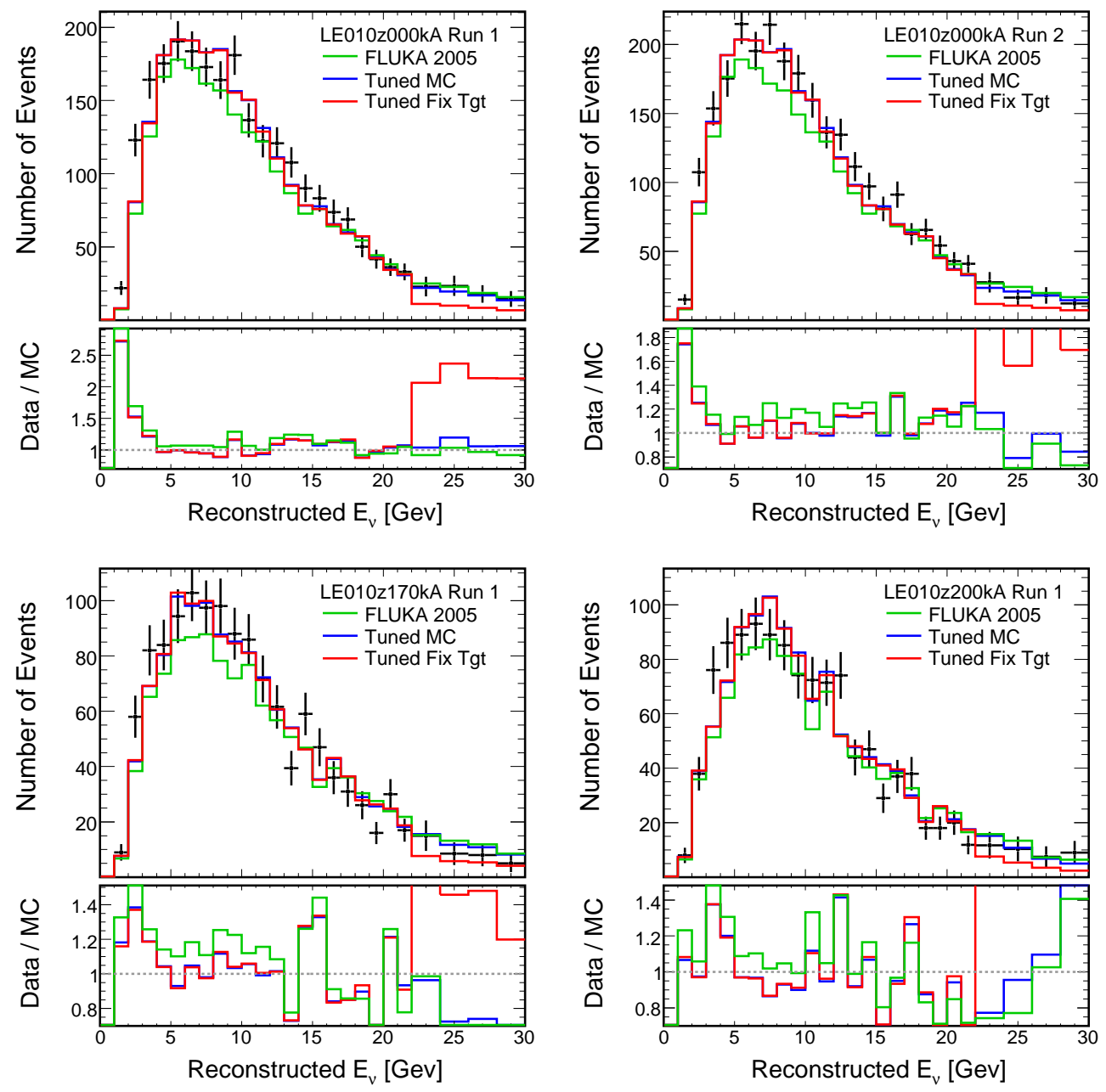

Figure 5.12: Anti-neutrino energy spectrum for the $\bar{\nu}_{\mu}$ charged current sample in the Near Detector. Black points are the data, green lines are the untuned Monte Carlo based on FLUKA, the blue is the result after the fit and red shows a fit with the target position fixed. 

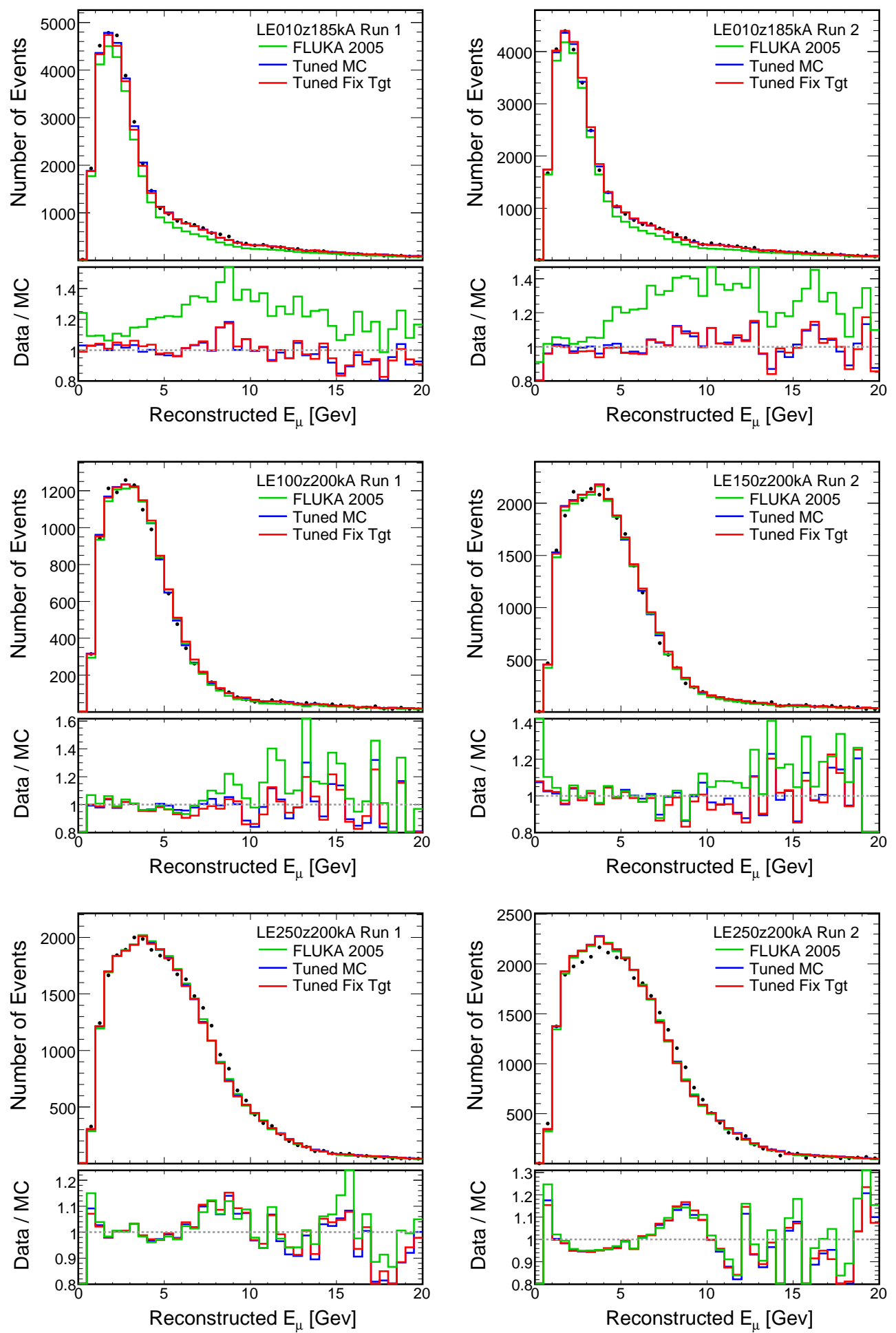

Figure 5.13: Muon energy spectrum for the $\nu_{\mu}$ charged current sample in the Near Detector. Black points are the data, green lines are the untuned Monte Carlo based on FLUKA, the blue is the result after the fit and red shows a fit with the target position fixed. 

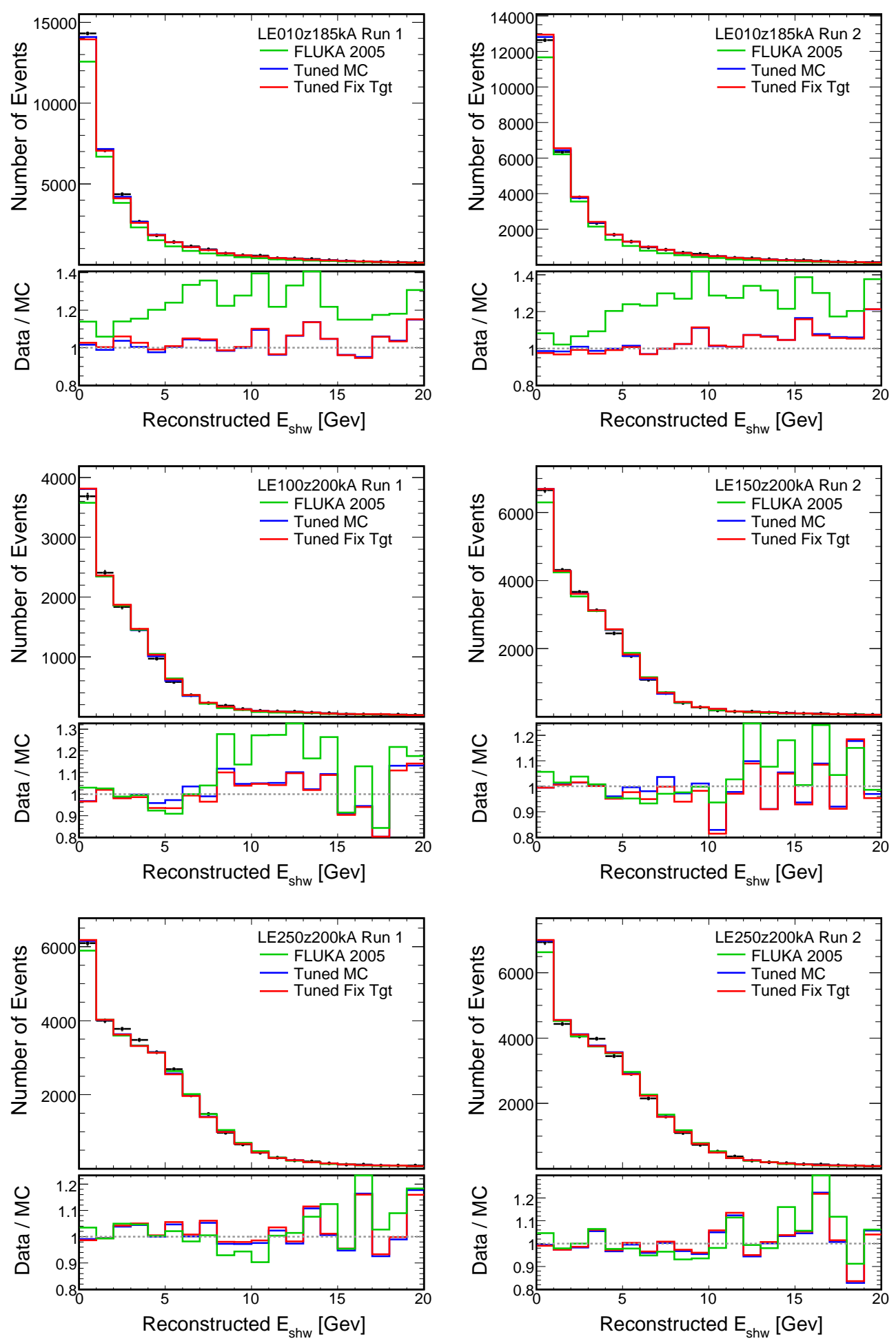

Figure 5.14: Shower energy spectrum for the $\nu_{\mu}$ charged current sample in the Near Detector. Black points are the data, green lines are the untuned Monte Carlo based on FLUKA, the blue is the result after the fit and red shows a fit with the target position fixed. 


\begin{tabular}{llcccc}
\hline \hline Beam & NuMI & $\begin{array}{c}\chi^{2} \\
\text { Configuration }\end{array}$ & $\begin{array}{c}\chi^{2} \\
\text { Operation }\end{array}$ & $\begin{array}{c}\chi^{2} \\
\text { (No Tuning) }\end{array}$ & $\begin{array}{c}\text { Degrees of } \\
\text { (funed MC) }\end{array}$ \\
\hline$\nu_{\mu}$ LE010z0kA & RunI & 538.0 & 95.3 & $(100.9)$ & 69 \\
$\nu_{\mu}$ LE010z0kA & RunII & 620.5 & 75.5 & $(79.1)$ & 69 \\
$\nu_{\mu}$ LE010z170kA & RunI & 427.8 & 87.4 & $(89.9)$ & 70 \\
$\nu_{\mu}$ LE010z185kA & RunI & 2387.9 & 211.6 & $(246.9)$ & 160 \\
$\nu_{\mu}$ LE010z185kA & RunII & 1737.5 & 181.7 & $(209.8)$ & 159 \\
$\nu_{\mu}$ LE010z200kA & RunI & 352.6 & 89.8 & $(88.8)$ & 69 \\
$\nu_{\mu}$ LE100z200kA & RunI & 327.0 & 170.9 & $(204.0)$ & 158 \\
$\nu_{\mu}$ LE150z200kA & RunII & 376.0 & 202.2 & $(239.5)$ & 159 \\
$\nu_{\mu}$ LE250z200kA & RunI & 249.7 & 233.2 & $(254.2)$ & 158 \\
$\nu_{\mu}$ LE250z200kA & RunII & 395.7 & 332.6 & $(340.9)$ & 160 \\
$\bar{\nu}_{\mu}$ LE010z0kA & RunI & 54.0 & 37.1 & $(37.7)$ & 28 \\
$\bar{\nu}_{\mu}$ LE010z0kA & RunII & 55.3 & 28.9 & $(29.5)$ & 28 \\
$\bar{\nu}_{\mu}$ LE010z170kA & RunI & 51.0 & 28.1 & $(29.0)$ & 28 \\
$\bar{\nu}_{\mu}$ LE010z185kA & RunI & 192.5 & 71.4 & $(71.5)$ & 67 \\
$\bar{\nu}_{\mu}$ LE010z185kA & RunII & 172.7 & 79.2 & $(80.8)$ & 67 \\
$\bar{\nu}_{\mu}$ LE010z200kA & RunI & 37.0 & 33.8 & $(34.1)$ & 28 \\
$\bar{\nu}_{\mu}$ LE100z200kA & RunI & 39.3 & 40.0 & $(40.2)$ & 28 \\
$\bar{\nu}_{\mu}$ LE150z200kA & RunII & 44.3 & 36.5 & $(35.4)$ & 28 \\
$\bar{\nu}_{\mu}$ LE250z200kA & RunI & 33.8 & 30.6 & $(30.1)$ & 28 \\
$\bar{\nu}_{\mu}$ LE250z200kA & RunII & 50.5 & 46.6 & $(45.6)$ & 28 \\
Parameter penalty terms & - & - & 32.9 & $(2.5)$ & - \\
Hadron Production penalty terms & - & - & 20.1 & $(15.4)$ & - \\
\hline Total & - & 8143.1 & 2165.6 & $(2305.9)$ & 1589 \\
\hline \hline
\end{tabular}

Table 5.3: Contributions to the $\chi^{2}$ from each beam configuration. Values in parentheses are obtained from a fit where the target positions fixed. 


\begin{tabular}{|c|c|c|c|c|c|c|}
\hline \multicolumn{4}{|c|}{$\pi^{+}$} & & \multicolumn{2}{|l|}{$K^{+}$} \\
\hline & Best F & it Value & Error & & Best Fit Value & Error \\
\hline par0 & 1.01 & $(0.12)$ & 0.06 & par6 & $-0.67(3.31)$ & 0.74 \\
\hline par1 & -8.07 & $(-4.28)$ & 0.25 & par7 & $20.50 \quad(12.52)$ & 1.80 \\
\hline par2 & 1.11 & $(0.93)$ & 0.01 & par8 & $1.38(1.46)$ & 0.05 \\
\hline par3 & -0.56 & $(0.17)$ & 0.03 & par9 & $0.75 \quad(0.57)$ & 0.16 \\
\hline par4 & 1.13 & $(0.96)$ & 0.01 & par10 & $0.92 \quad(0.96)$ & 0.09 \\
\hline par5 & -0.04 & $(0.69)$ & 0.05 & par11 & $2.14 \quad(2.02)$ & 0.28 \\
\hline \multicolumn{4}{|c|}{$\pi^{-}$} & & \multicolumn{2}{|l|}{$K^{-}$} \\
\hline par12 & 1.07 & $(1.06)$ & 0.01 & par14 & $(0.70)$ & 0.069 \\
\hline par13 & -0.95 & $(-0.94)$ & 0.02 & par15 & $(0.78)$ & 0.205 \\
\hline
\end{tabular}

Table 5.4: Best fit values and $1 \sigma$ errors from the fit for the hadron production parameters. The numbers indicate the change required to shift the Monte Carlo to match the data. The values in parenthesis are those from the fit where the target positions were fixed.

\subsubsection{Comments}

The fit is able to reduce the discrepancy between the data and the Monte Carlo to less than $\sim 5 \%$ in all the beams. The fit favors large shifts for the target parameters. In particular the target positions for the le100z200i and le150z200i data are shifted by more than $3 \mathrm{~cm}$ from the nominal position. It is possible that the target parameter is covering for some aspect of the beam that is not being properly modeled. The other parameters do not seem to have been pulled far from within the estimated uncertainty.

\subsubsection{Effect on Hadron Production}

By observing the high energy of tail of the LE configuration and the Horn Off data, it can be seen that the largest discrepancy between data and Monte Carlo is a result of unfocused hadrons. To compensate for this, the fit should increase the relative number of hadrons at low $p_{T}$. Figure 5.15 shows how the number of hadrons is modified by the fit as a function of $p_{T}$ and $p_{Z}$ relative to FLUKA. It confirms the expectation at low $p_{T}$, and indicates that small corrections were made to the momentum space important for the LE configuration. 


\begin{tabular}{llccc}
\hline \hline Beam & NuMI & Best-Fit $[\mathrm{cm}]$ & Error $[\mathrm{cm}]$ & $1 \sigma[\mathrm{cm}]$ \\
Configuration & Operation & & & \\
\hline LE010z & RunI & +1.64 & 0.36 & 1.0 \\
LE100z & RunI & -3.45 & 0.56 & 1.0 \\
LE250z & RunI & +0.68 & 0.69 & 1.0 \\
LE010z & RunII & -1.57 & 0.39 & 1.0 \\
LE150z & RunII & -3.60 & 0.53 & 1.0 \\
LE250z & RunII & +0.45 & 0.68 & 1.0 \\
\hline \hline
\end{tabular}

Table 5.5: Best fit values and errors from the fit for the target positions.

\begin{tabular}{lrrrr}
\hline \hline & Best-Fit & Error & (Fixed tgt) & $1 \sigma$ \\
\hline$M_{A}$ for QE & $+2.8 \%$ & $2.2 \%$ & $(+6.6 \%)$ & $15 \%$ \\
$M_{A}$ for RES & $-2.5 \%$ & $1.2 \%$ & $(-2.1 \%)$ & $15 \%$ \\
$\bar{\nu}_{\mu}$ CC cross-section & 0.95 & 0.02 & $(0.92)$ & 1.0 \\
NC normalization for $\nu_{\mu}$ & $+14.0 \%$ & $9.7 \%$ & $(+16.4 \%)$ & $30 \%$ \\
NC normalization for $\bar{\nu}_{\mu}$ & $-36.8 \%$ & $25.3 \%$ & $(-37.4 \%)$ & $30 \%$ \\
Shower energy offset & $-23.7 \mathrm{MeV}$ & $6.2 \mathrm{MeV}$ & $(-18.5 \mathrm{MeV})$ & $50 \mathrm{MeV}$ \\
Shower energy scale & $-0.34 \sigma$ & $0.03 \sigma$ & $(-0.34 \sigma)$ & $1.0 \sigma$ \\
Muon energy scale & $-0.50 \%$ & $0.13 \%$ & $(-0.85 \%)$ & $2 \%$ \\
\hline \hline
\end{tabular}

Table 5.6: Best fit values and 1 errors from the fit for the cross-section and detector errors. Numbers in parentheses are obtained from a fit where the target positions are fixed. The $1 \sigma$ column indicates the estimated uncertainties that were used to constrain the fit. 

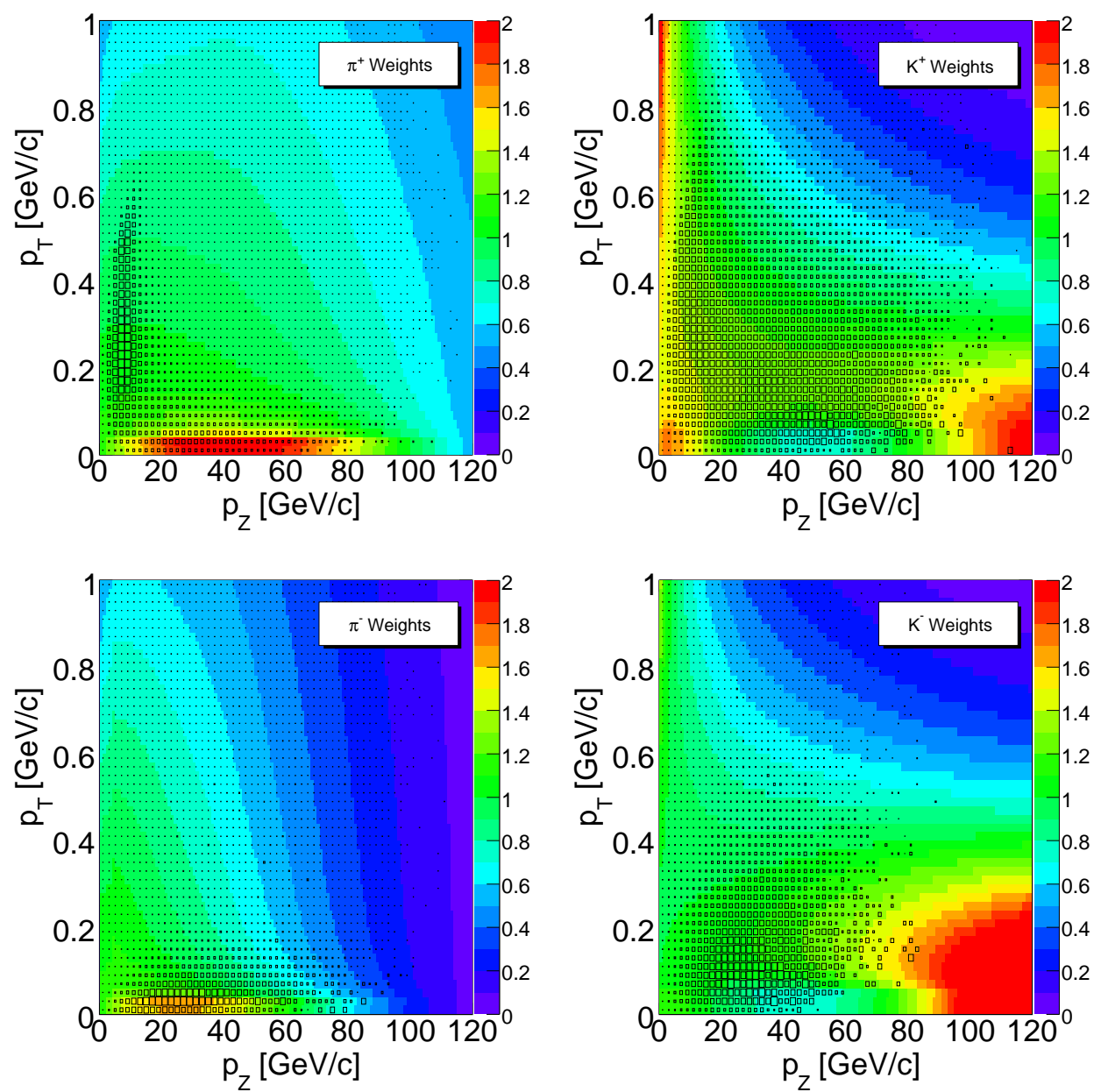

Figure 5.15: The color indicates the weights given to hadrons as a result of the fit as a function of $p_{T}$ and $p_{Z}$. The boxes show the probability that a neutrino will create a neutrino in the Near Detector for the le010z185i beam configuration. 


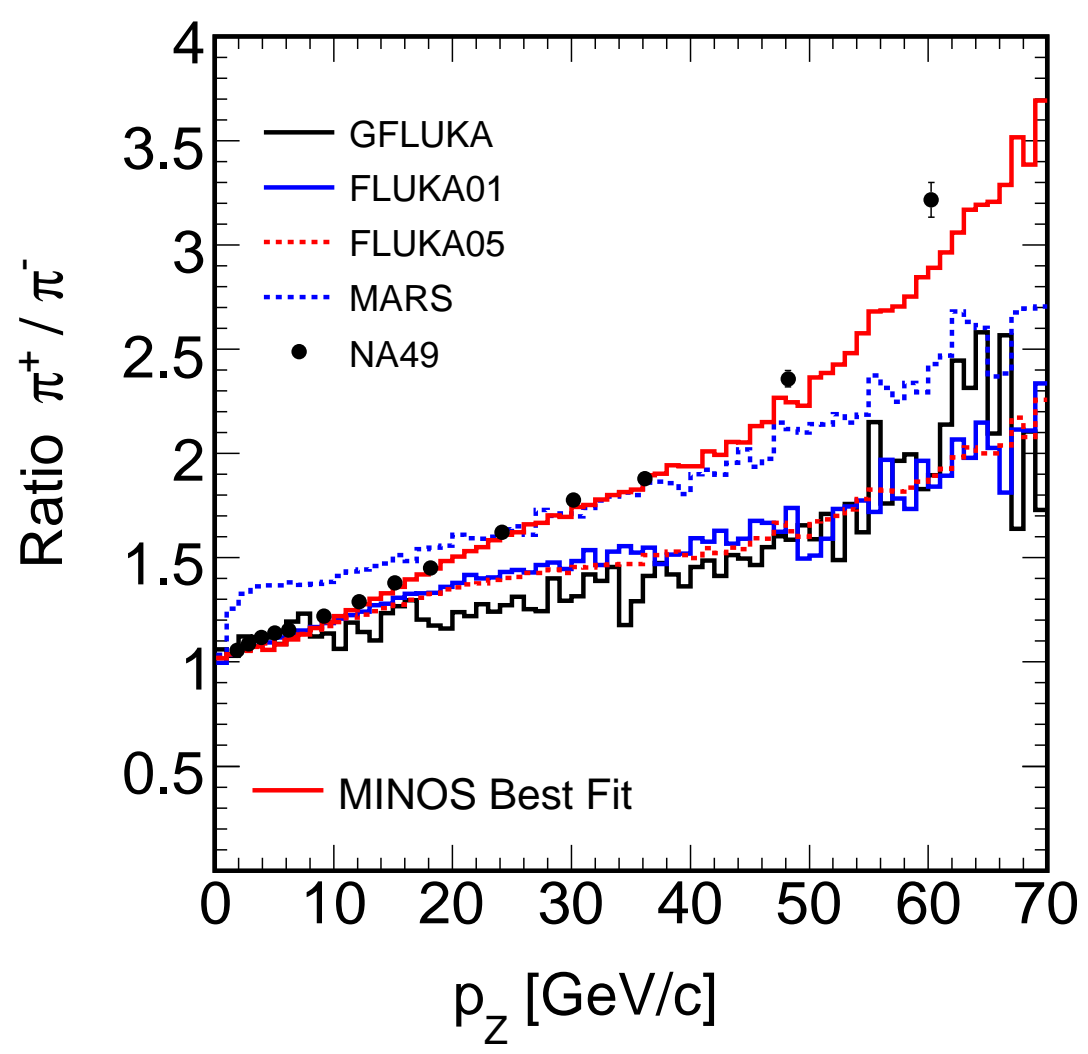

Figure 5.16: The $\pi^{+} / \pi^{-}$of the fit compared to NA49 data and various other models.

\subsubsection{Comparison to External Data}

As noted earlier, there is very little data to constrain the hadron production. Figure 5.6.3 shows the $\pi^{+} / \pi^{-}$ratio integrated over $p_{T}$ compared to the NA49 data. Figure 5.17 compare the ratios between $K^{+} / K^{-}, K^{+} / \pi^{+}$, and $K^{-} / \pi^{-}$between the fit and other models. A comparison can also be done (see Figure 5.18) with preliminary data from the MIPP experiment [72] for the particle production ratios. Comparisons of the $K^{+} / K^{-}$, $K^{-} / \pi^{-}$, and $K^{+} / \pi^{+}$ratios show good agreement with these results. 

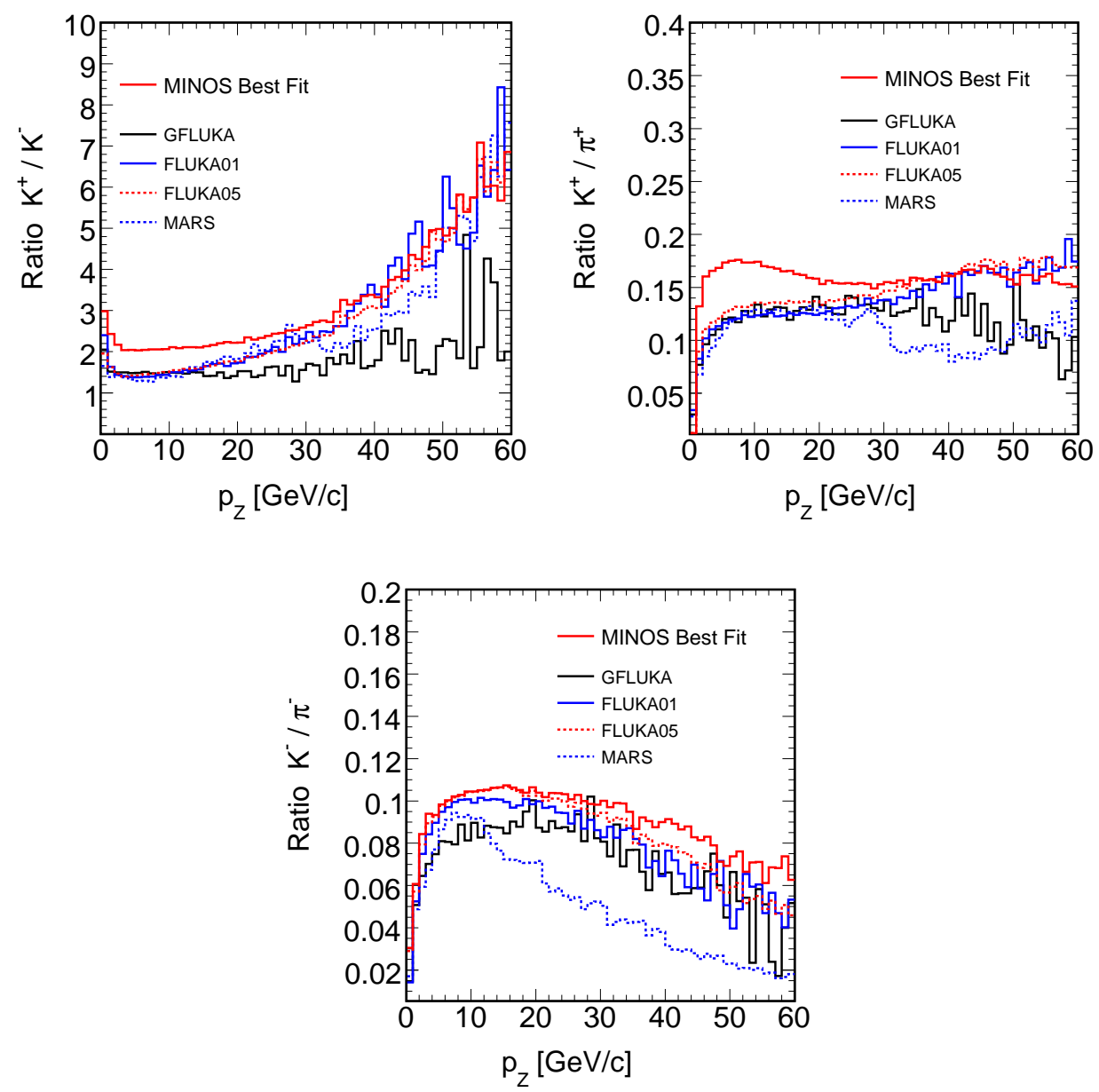

Figure 5.17: A comparison of particle production ratios between the fit and different models. 

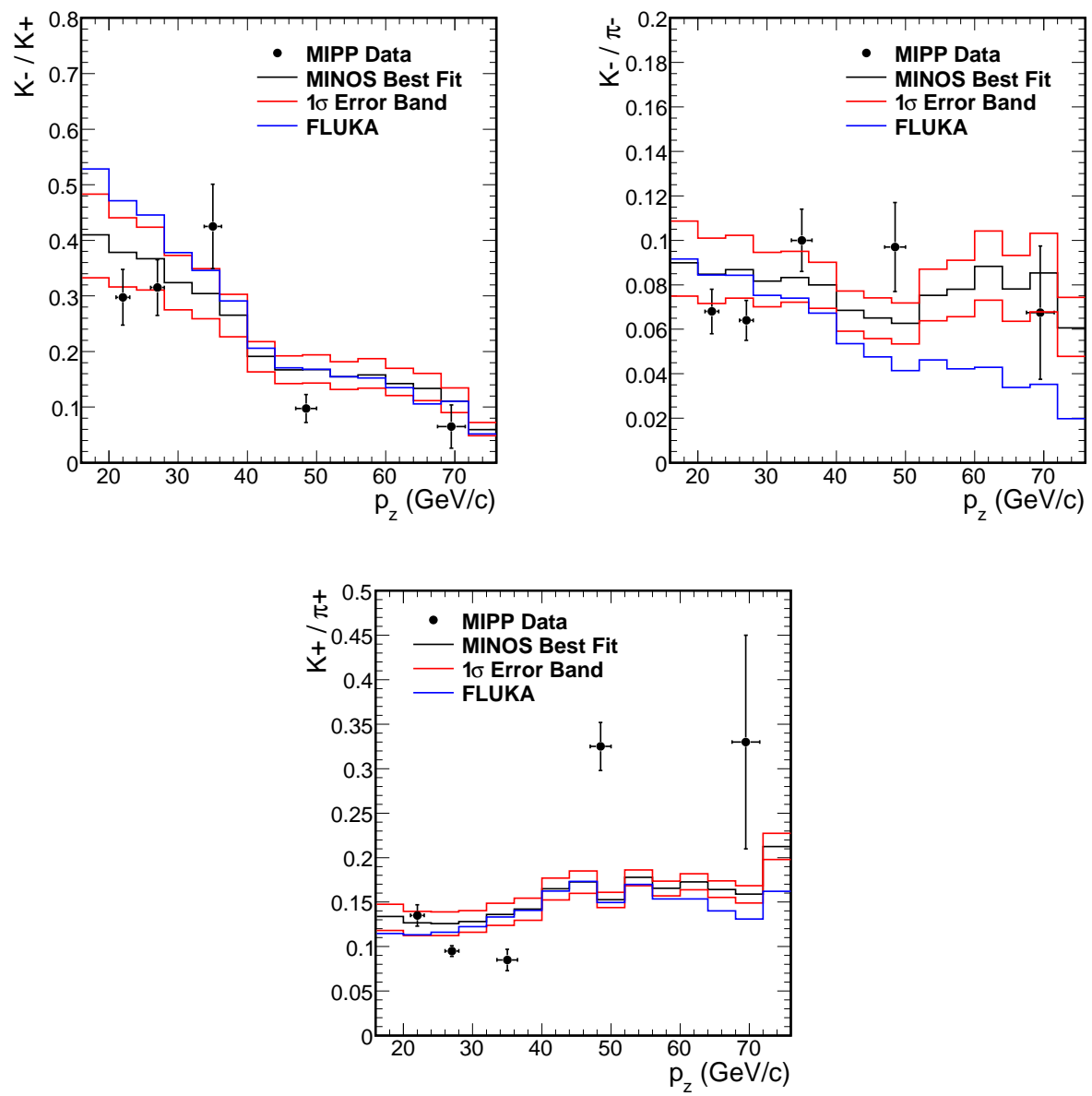

Figure 5.18: A comparison of particle production ratios from the Near Detector fit and preliminary data from the MIPP experiment. 


\subsection{Uncertainty on the Flux after the Fit}

What are the remaining uncertainties in the flux prediction after the fit has been performed? Uncertainties not explicitly included the fit must be taken into account such as those due to beam focusing and alignment (see Section 3.1.6). There also remain statistical uncertainties on the parameters after the fit.

\subsubsection{Uncertainty from Hadron Production after the Fit}

The residual uncertainty from hadron production from the fit is calculated from the errors on those parameters and their correlations. A collection of 750 random sets of hadron production parameters were generated centered on the values obtained from the fit, with a variance reflecting their correlated errors. A Cholesky decomposition of the covariance matrix into a lower triangular matrix gives:

$$
M=A \dot{A}^{T},
$$

where $M$ is the covariance matrix and $A$ the lower triangular matrix. From the $A$, a random vector of the parameters, $V$, can then be generated via:

$$
V=\Gamma+A Z
$$

where $\Gamma$ is a vector of the best fit points and $Z$ is a vector of normally distributed random numbers with mean zero. Each parameter will be centered on the best fit value from the fit and the width will include correlations from other parameters.

For each of the 750 random sets the Far Detector and Near Detector flux is found by reweighting the same Monte Carlo sample. The error for each energy bin is found by taking the maximum deviation of the bin content for each of the 750 possible values. The errors are then doubled to account for the difference seen in the high energy tail above 10 
$\mathrm{GeV}$ between the fit where the target positions were fixed and where they were allowed to float. Figures 5.19 and 5.20 show the calculated uncertainty.

\subsubsection{Total Uncertainty}

The total error is found by adding in quadrature the beam alignment errors, the shift caused by the fitted target position, and the hadron production errors from the previous section. The final errors on the $\nu_{\mu}$ Far/Near ratio for different beam configurations are shown in Figure 5.21. These figures demonstrate that the uncertainty on the flux can be reduced to only a few percent. At energies below $10 \mathrm{GeV}$ the dominant uncertainty to the flux comes from the beam parameters and the target position. Hadron production accounts for the uncertainty at higher energies.

\subsubsection{Correlations on the Fit Parameters}

In a fit with a large number of parameters there are bound to be parameters that are highly correlated with each other. Figure 5.22 shows the correlation matrix of the fit parameters. Significant correlation is seen between the parameters governing hadron production. The cause of this is the particular parameterization chosen. Other large correlations are seen between the two cross-section parameters RES $M_{A}$ and QE $M_{A}$ and between QE $M_{A}$ and the shower energy offset.

\subsubsection{Impact on the Flux}

The impact on the neutrino flux is found by applying the target and hadron production weights to the Monte Carlo simulation. A comparison of the uncertainty on the Far over Near ratio can be done for before and after the fit. Figure 5.23 shows this comparison, where the uncertainty before the fit is found taking the spread of different hadron production models. The fit has reduced the uncertainty over the entire energy spectrum, except 

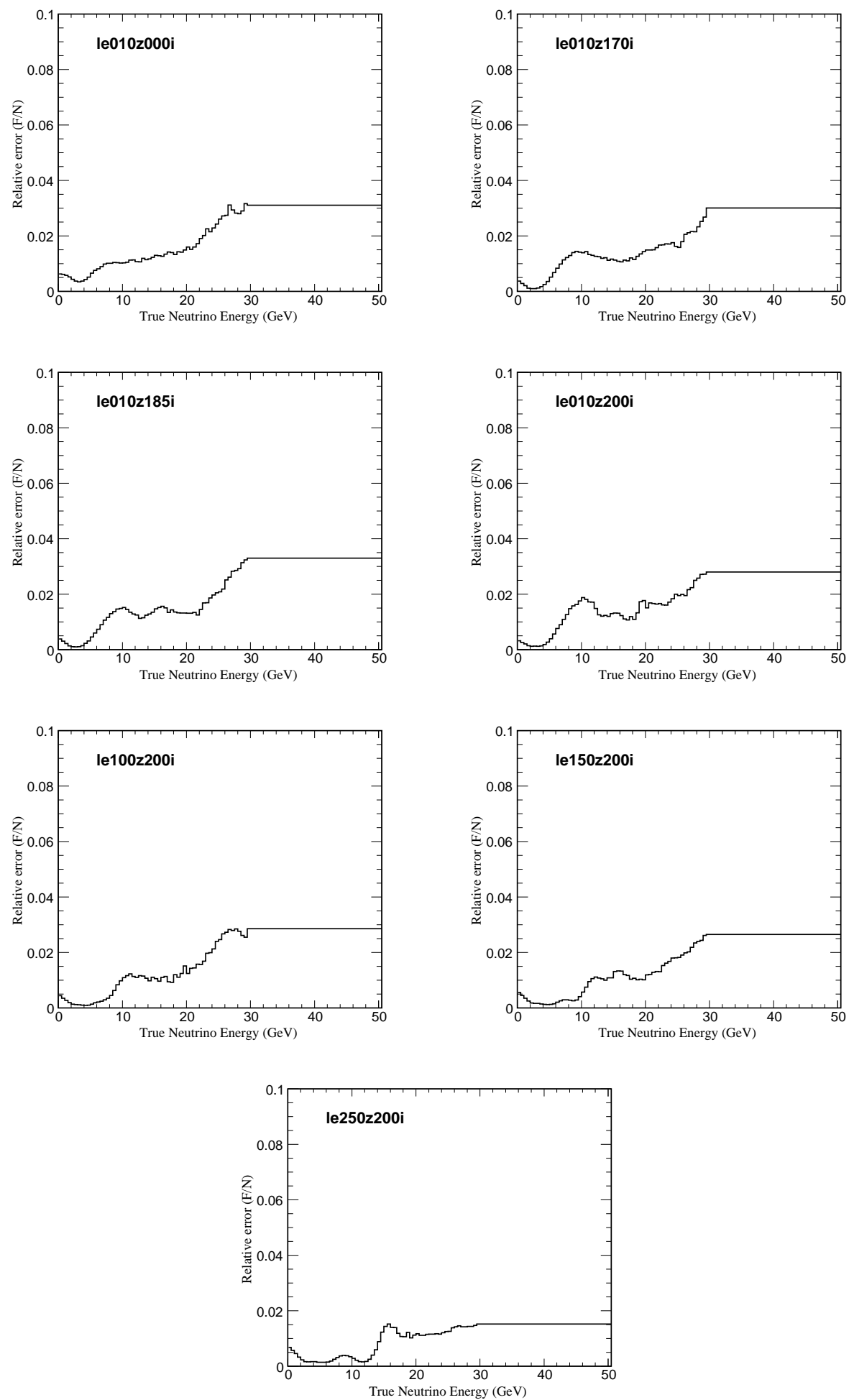

Figure 5.19: Systematic uncertainty on the $\nu_{\mu}$ flux from hadron production on the Far/Near ratio for different beam configurations. 

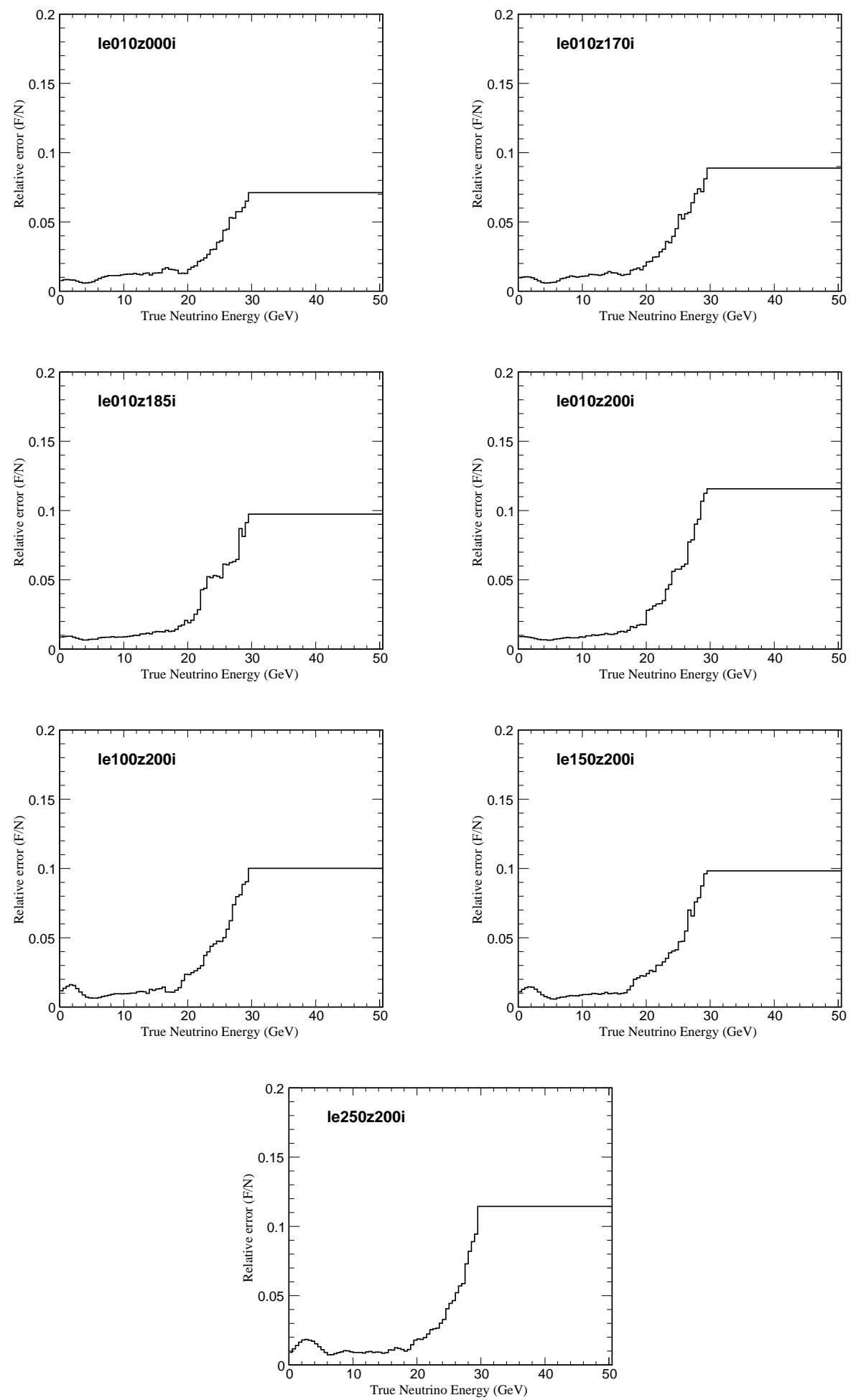

Figure 5.20: Systematic uncertainty on the $\bar{\nu}_{\mu}$ flux from hadron production the Far/Near ratio for different beam configurations. 

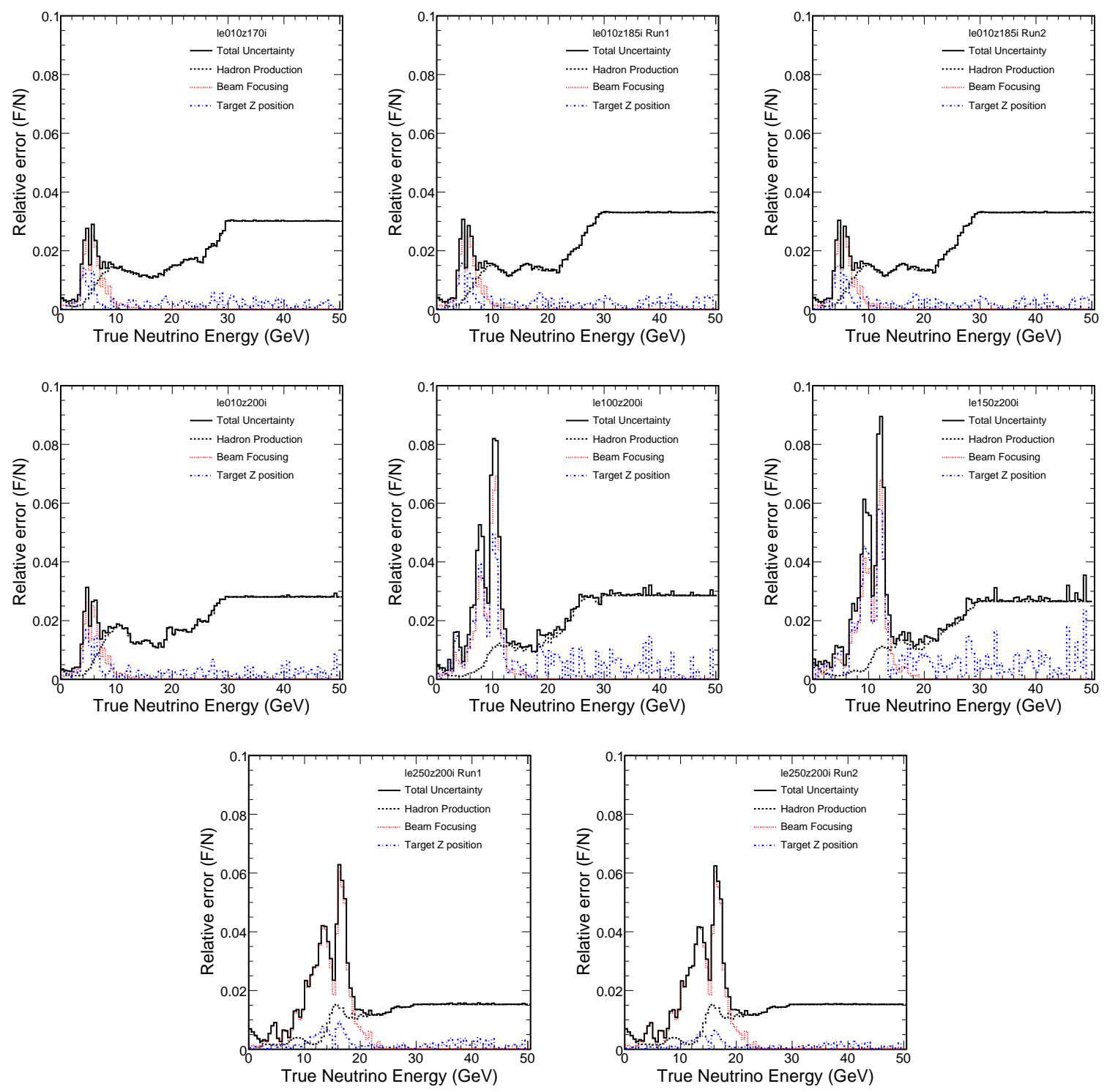

Figure 5.21: Total systematic uncertainty on the $\nu_{\mu}$ flux on the Far/Near ratio for different beam configurations. 


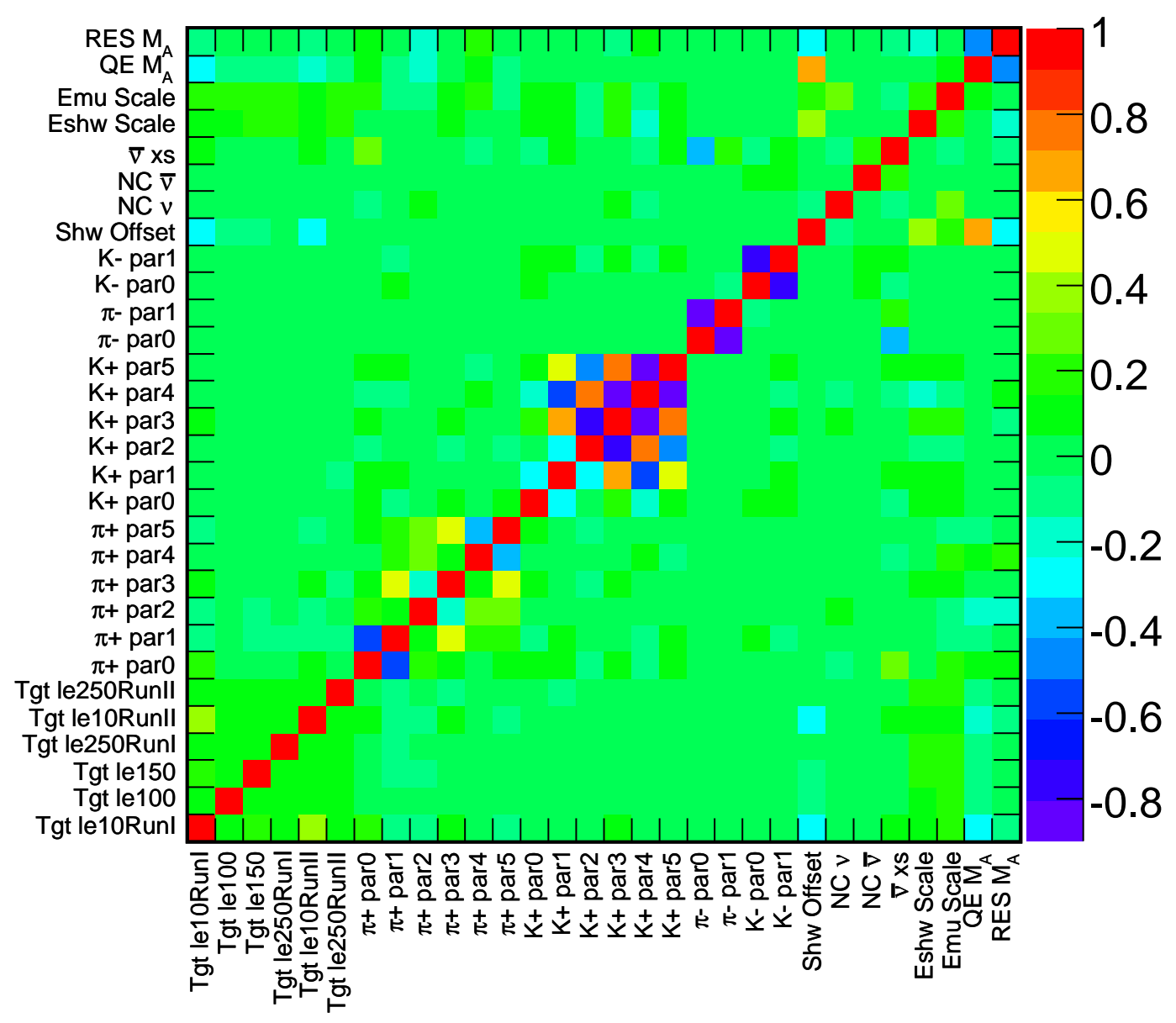

Figure 5.22: The correlation matrix of all the free parameters from the fit to the Near Detector data. 
for the falling edge of the peak which occurs at where hadrons traverse the boundaries of the focusing horns. 


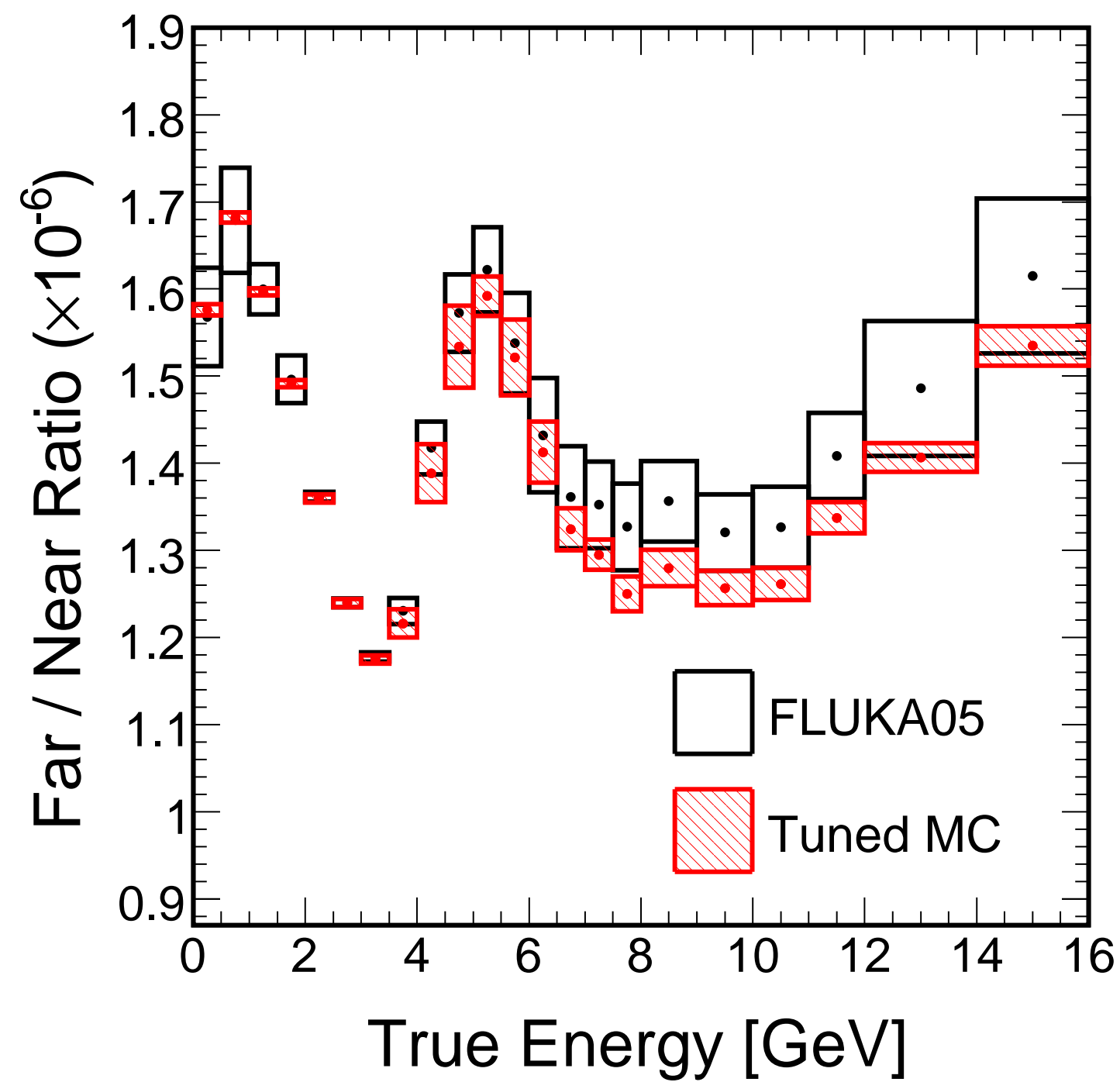

Figure 5.23: A comparison of the systematic uncertainty before and after the fit. 


\section{Chapter 6}

\section{Near Detector Data and}

\section{Extrapolation to the Far Detector}

With the Monte Carlo tuning described in Chapter 5, a direct comparison of all of the data in the Near Detector can be made. This serves as an important check for two reasons. First, the tuning procedure should be checked with different physics distributions to see if any problems or discrepancies result from the tuning. Second, this tuning will serve as a unoscillated prediction for the Far Detector. Any remaining discrepancies could potentially bias the prediction in the Far Detector. A detailed comparison will only be done for the data sets that will be used in the oscillation analysis: the le010z185i (LE) and le250z200i (HE) configurations.

\subsection{Event Selection and Data/MC comparisons}

\subsubsection{Pre-Selection Cuts}

The following criteria were determined by the collaboration at large to provide a standard sample of CC-like events selected with high efficiency. 
- Data quality - A list of bad runs was rejected based on a run by run scan of the online monitoring files. An entire run was flagged as bad if at any time in the run there were problems.

- Good beam - Require that the spill come from from a time with reliable beam data.

- Coil current - Require that the current in the coil be at the nominal value and that the data was taken with the field orientation in which negative particles are focused.

- Has a track - Each event is required to have a reconstructed track. The track is required to pass the track fitting algorithm or satisfy the track reclamation cut. The track reclamation cut is used to reclaim tracks that fail the track fitter in the data. It can reclaim tracks for which the track fitter failed to converge, but the momentum can be reliably reconstructed from range. This reduces a bias seen in the track fitter where more Near Detector Data events were seen to fail compared to Monte Carlo.

- Negative curvature - only $\nu_{\mu}$ events are examined since $\bar{\nu}_{\mu}$ events will be considered in a separate analysis.

- Fiducial Volume - The fiducial volume is defined in the Near Detector by requiring the $z$ position of the vertex to be between planes 13 and 68 and the transverse position of the vertex to be within 0.8 meters of the beam center at the upstream end of the detector.

In addition there is a selection procedure described in Chapter 7 based on event characteristics to reject $\mathrm{NC}$ events in which a track from the hadron shower is spuriously inferred to be a $\mu$. 


\subsubsection{Distributions Studied}

The Monte Carlo distributions shown in the following pages use the reweighting from the Near Detector fit described in the previous chapter. A CC-like selection cut has been applied to all of the plots except the CC discriminator. Six different groups of plots will be examined:

- Energy Related Distributions - Includes the neutrino energy, muon energy from curvature and range, the shower energy, and the number of tracks and showers.

- Vertex and Track End - Contains event vertex for the $x, y, z$ directions, and the track end position in the $x, y, z$ directions.

- CC Selection - Contains the four variables used in the standard CC selection algorithm (see Section 7.2) including scintillator planes, mean pulse height, mean low pulse height / mean high pulse height, the transverse profile and the CC discrimination variable over all energies.

- CC Discriminator - Contains the charged current discrimination variable for different neutrino energy ranges.

- Miscellaneous - Contains the number of track strips, the cosine of the angle between the track and the beam direction, a measure of the reliability of the track curvature $(q / p) / \sigma_{q / p}$ where $q / p$ is the charge divided by the momentum, and the reconstructed $y$, where $y$ is the fraction of the neutrino energy that went into the hadronic shower.

\subsubsection{LE RunI Data/MC Comparison}

This section compares different distributions in the Near Detector for data and Monte Carlo for the LE RunI data comprising Figures 6.1 through 6.6. 

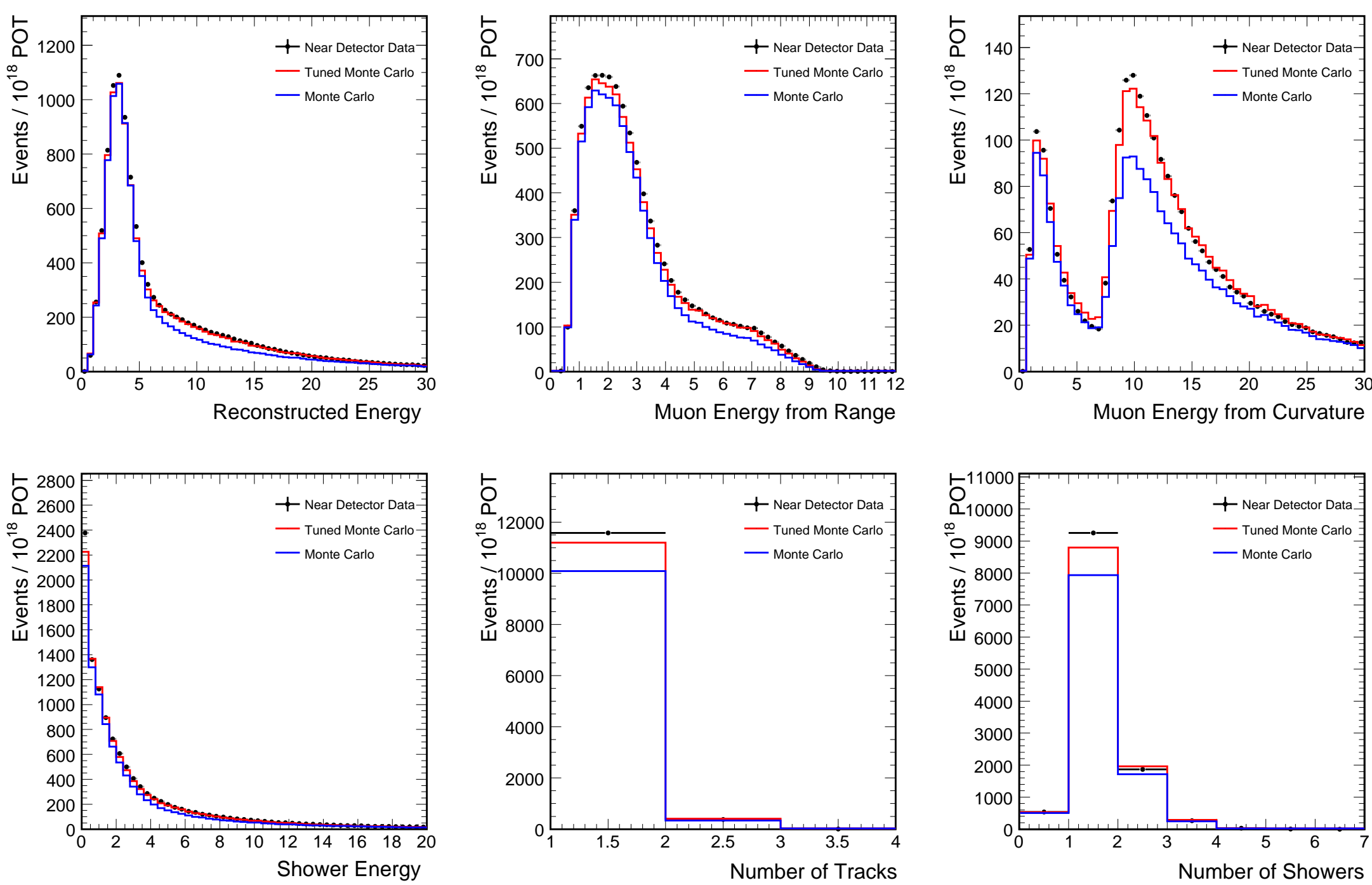

Figure 6.1: Energy plots for the LE RunI data and Monte Carlo. The plots are of neutrino energy (top left), muon energy from range (top middle), muon energy from range (top right), shower energy (bottom left), the number of tracks (bottom middle), the number of showers (bottom right). 

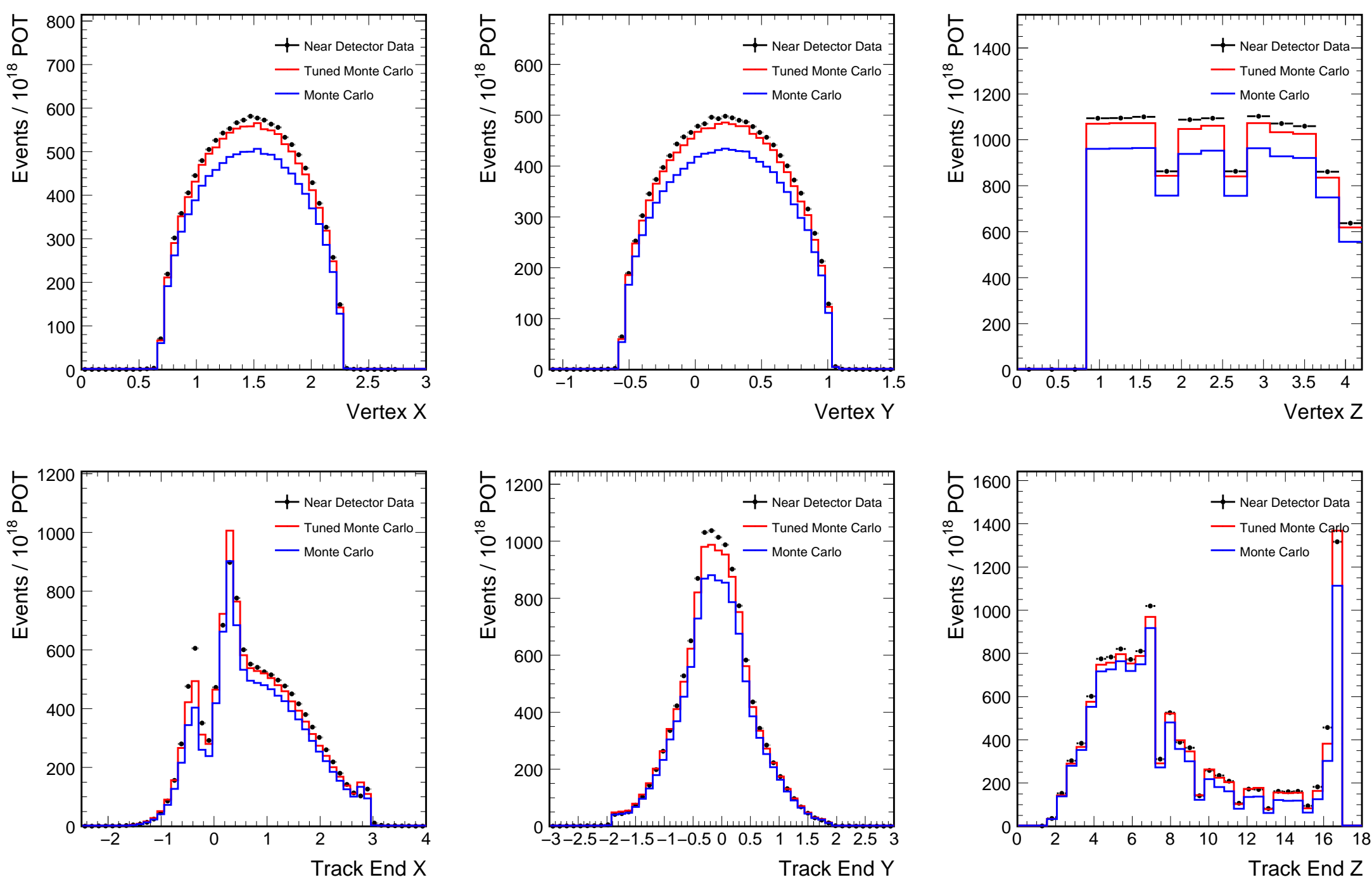

Figure 6.2: Vertex and track end plots for LE RunI data and Monte Carlo. The plots show the position of the event vertex for $x$ (top left), $y$ (top middle), and $z$ (top right), and the track end point for $x$ (bottom left), $y$ (bottom middle), and $z$ (bottom right). 

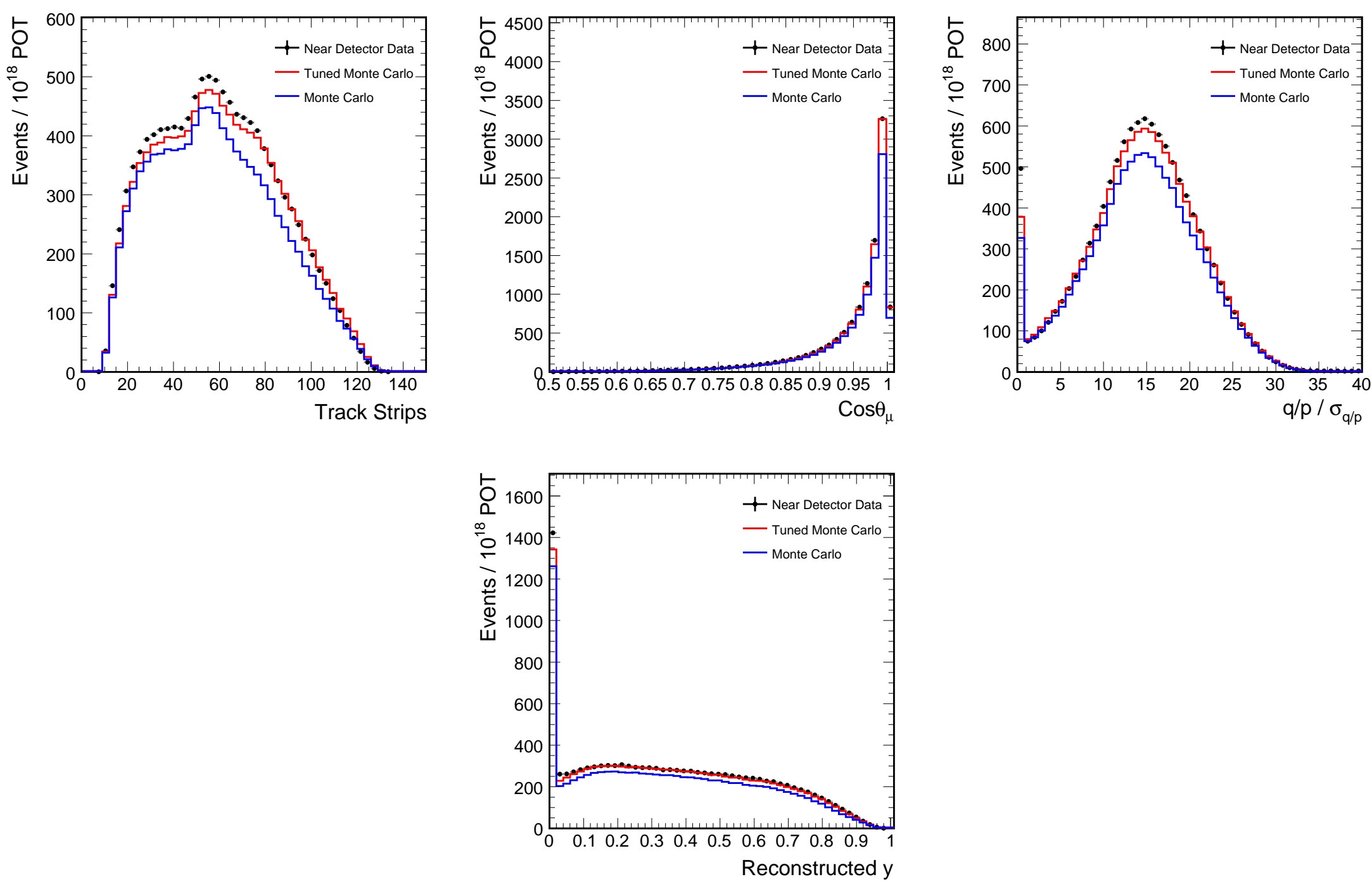

Figure 6.3: Miscellaneous plots for LE RunI data and Monte Carlo. The plots show the number of track strips (top left), the cosine of the angle between the track and beam direction (top middle), $(q / p) / \sigma_{q / p}$ (top right), reconstructed $y$ (bottom). 

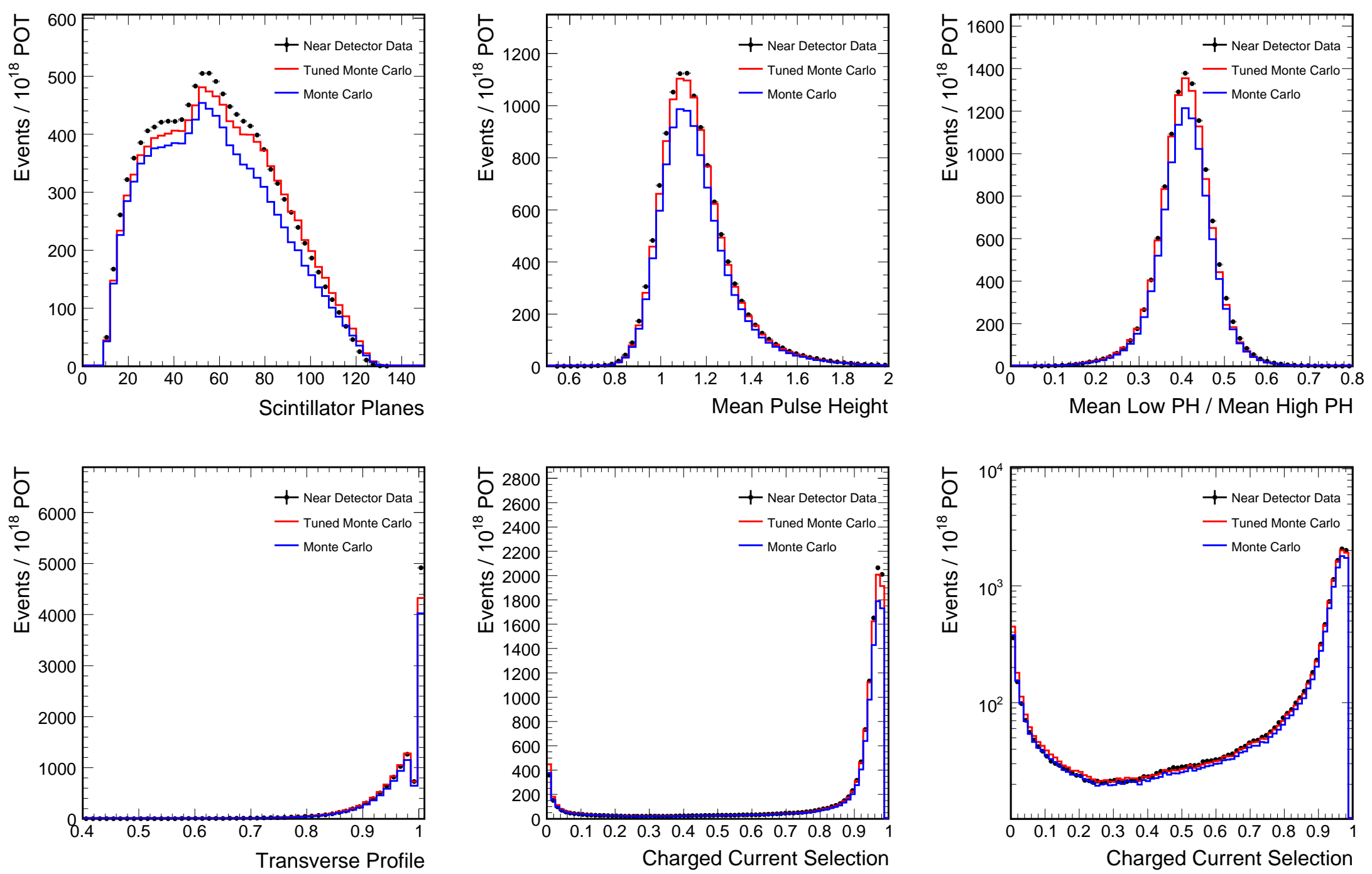

Figure 6.4: CC selection plots for LE RunI data and Monte Carlo. The plots show the number of scintillator planes (top right), the mean pulse height (top middle), low mean pulse height/high mean pulse height (top right), the transverse profile (bottom left), the CC discriminator (bottom middle), the CC discriminator on a log scale (bottom right). 

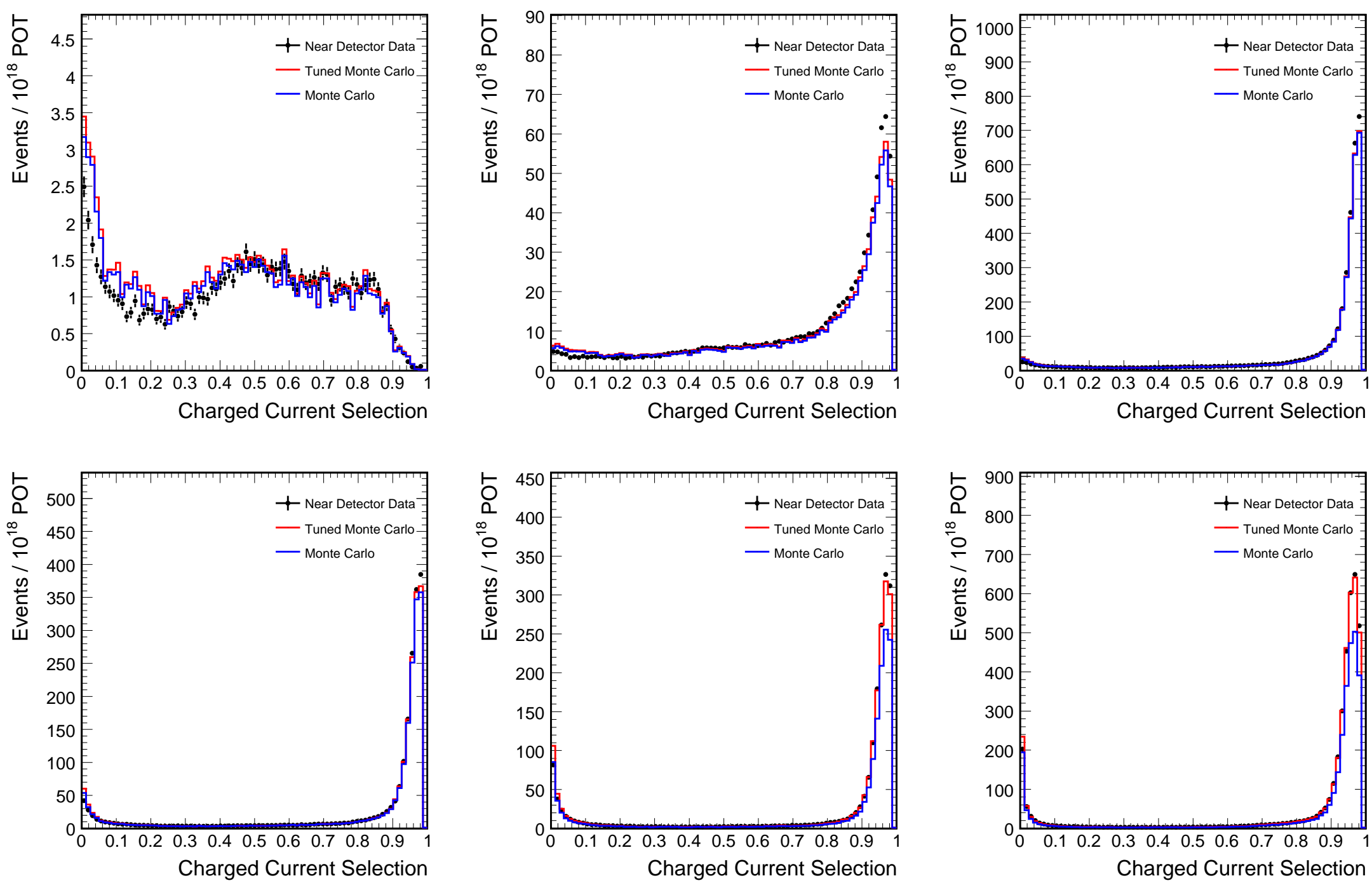

Figure 6.5: The CC discriminator for different neutrino energies for LE RunI data and Monte Carlo. The plots show the following energy ranges 0-1 GeV (top left), 1-2 GeV (top middle), 2-4 GeV (top right), 4-6 GeV (bottom left), 6-10 GeV (bottom middle), $>10 \mathrm{GeV}$ (bottom right) 

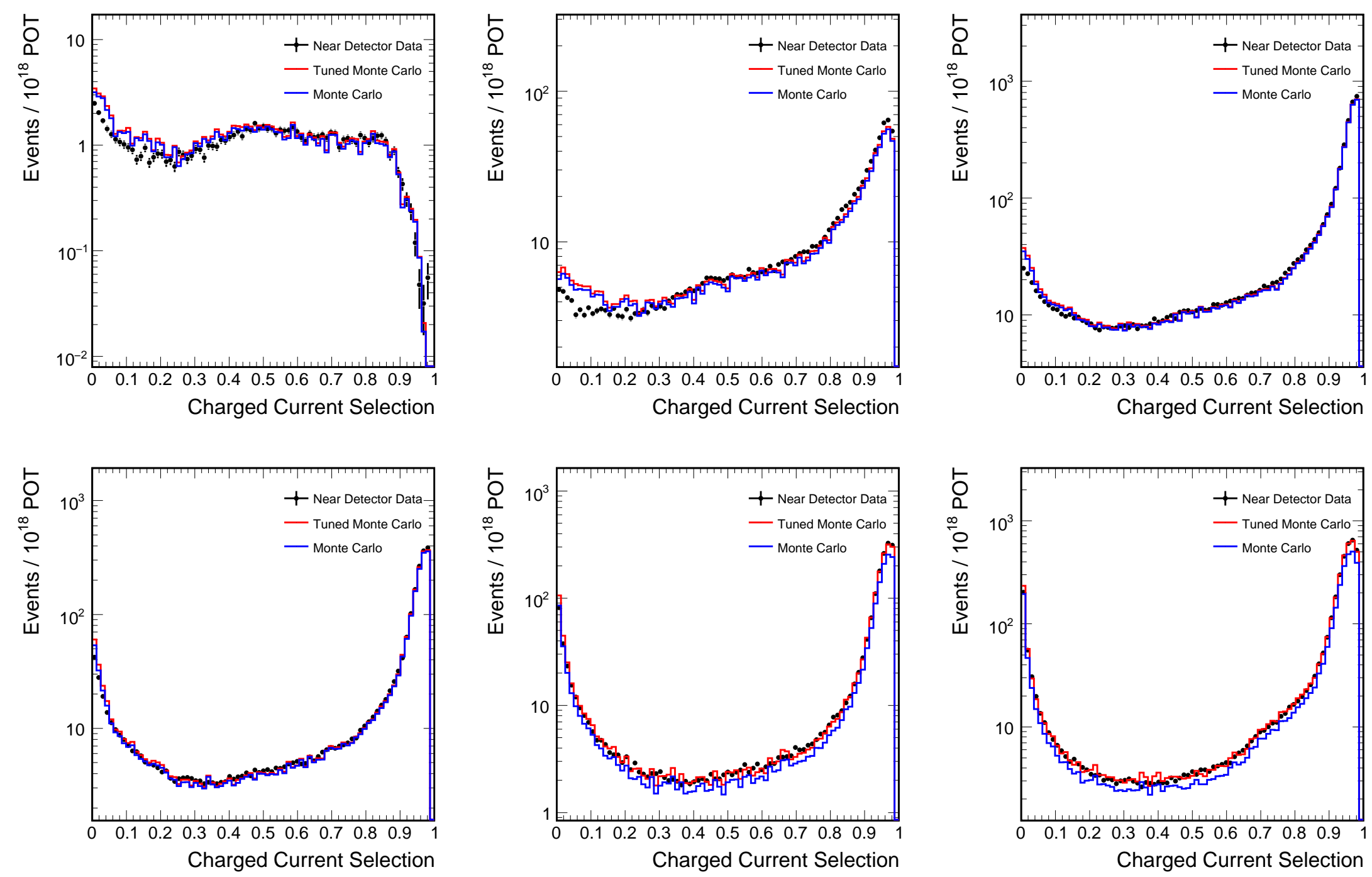

Figure 6.6: The charged current discriminator for different neutrino energies on a log scale for LE RunI data and Monte Carlo. The plots show the following energy ranges 0-1 GeV (top left), 1-2 GeV (top middle), 2-4 GeV (top right), 4-6 GeV (bottom left), 6-10 GeV (bottom middle), $>10 \mathrm{GeV}$ (bottom right). 


\subsubsection{LE RunII Data/MC Comparison}

This section compares different distributions in the Near Detector for data and Monte Carlo for the LE RunII comprising Figures 6.7 through 6.12. 

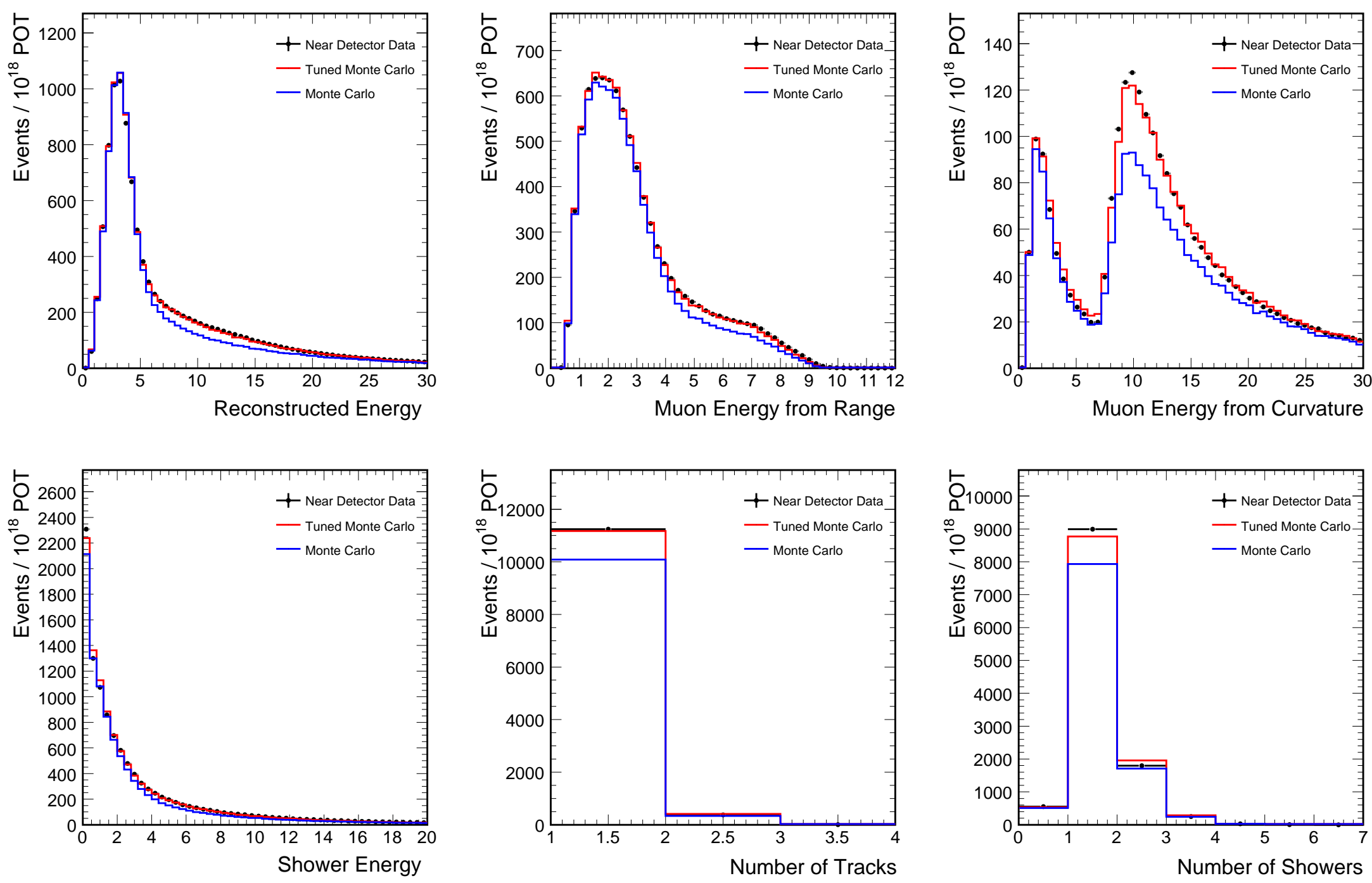

Figure 6.7: Energy plots for the LE RunII data and Monte Carlo. The plots are of neutrino energy (top left), muon energy from range (top middle), muon energy from range (top right), shower energy (bottom left), the number of tracks (bottom middle), the number of showers (bottom right). 

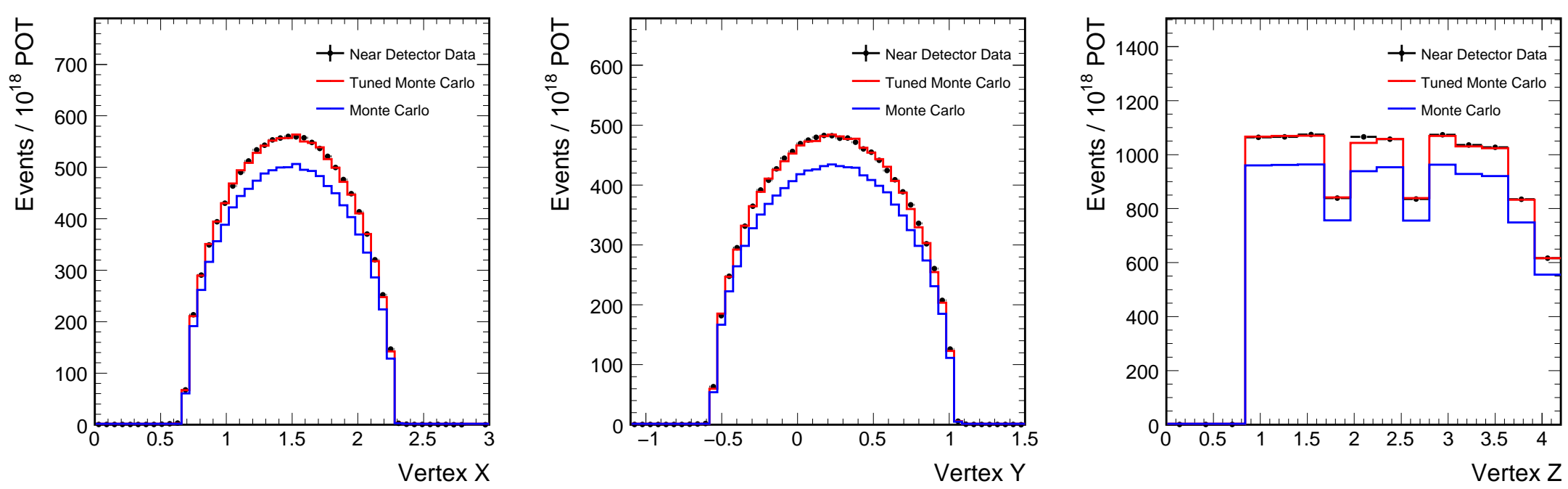

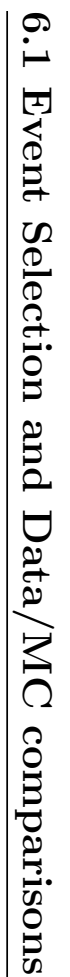
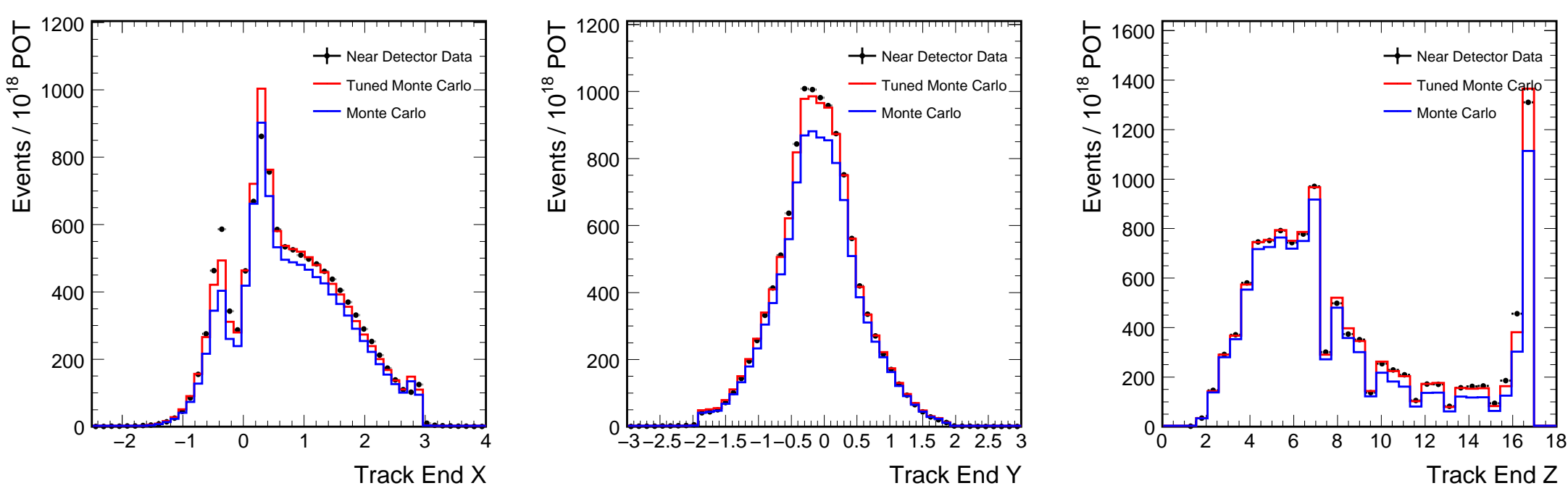

Figure 6.8: Vertex and track end plots for LE RunII data and Monte Carlo. The plots show the position of the event vertex for $x$ (top left), $y$ (top middle), and $z$ (top right), and the track end point for $x$ (bottom left), $y$ (bottom middle), and $z$ (bottom right). 

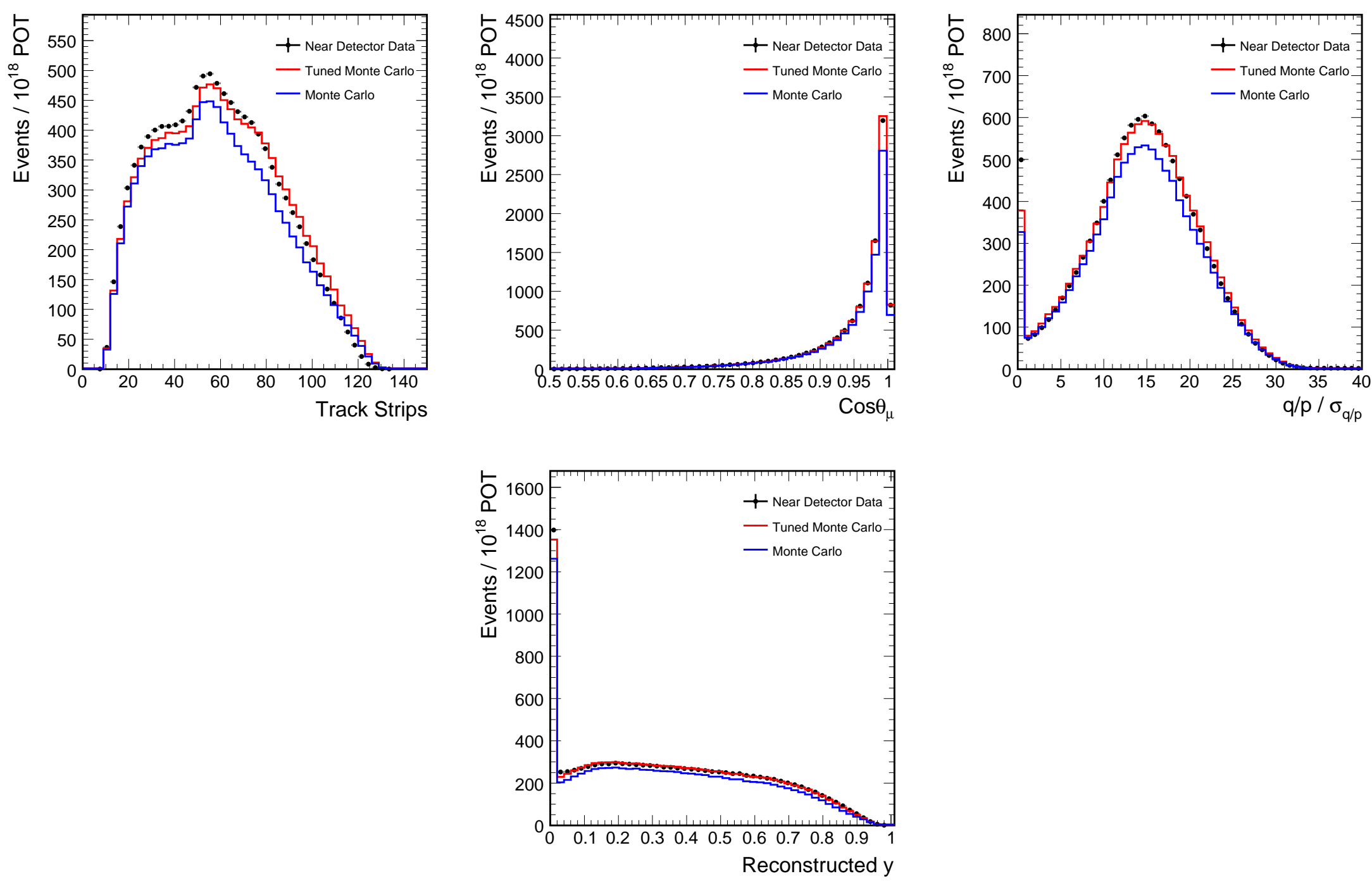

Figure 6.9: Miscellaneous plots for LE RunII data and Monte Carlo. The plots show the number of track strips (top left), the cosine of the angle between the track and beam direction (top middle), $(q / p) / \sigma_{q / p}$ (top right), reconstructed $y$ (bottom). 

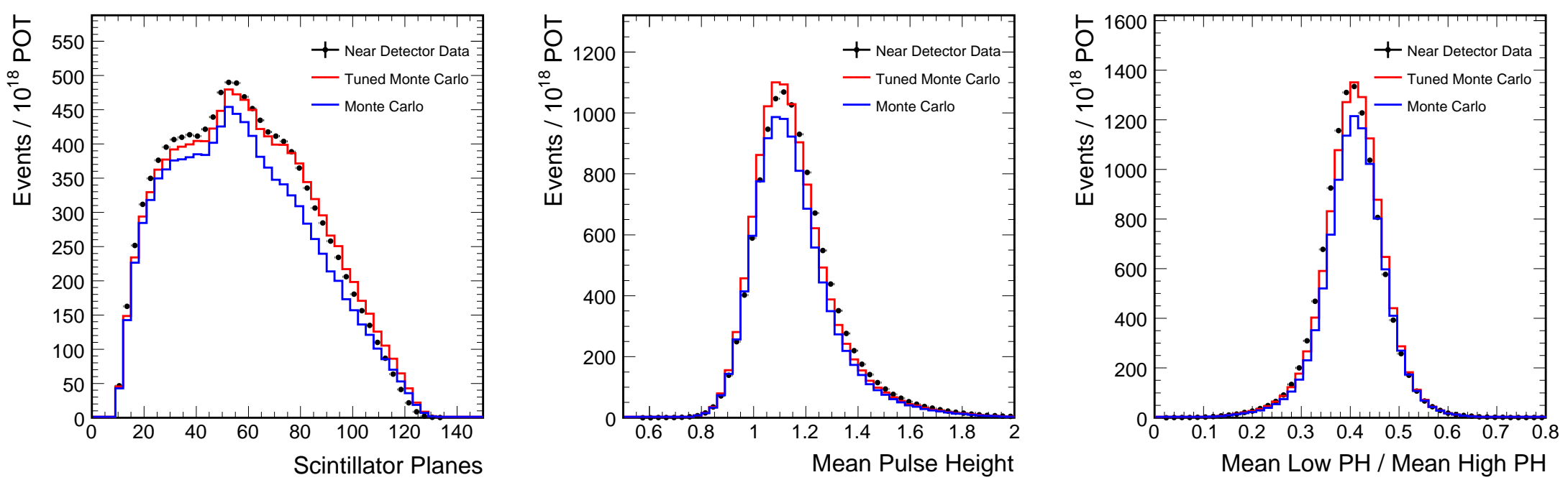

Figure 6.10: CC selection plots for LE RunII data and Monte Carlo. The plots show the number of scintillator planes (top right), the mean pulse height (top middle), low mean pulse height/high mean pulse height (top right), the transverse profile (bottom left), the CC discriminator (bottom middle), the CC discriminator on a log scale (bottom right). 

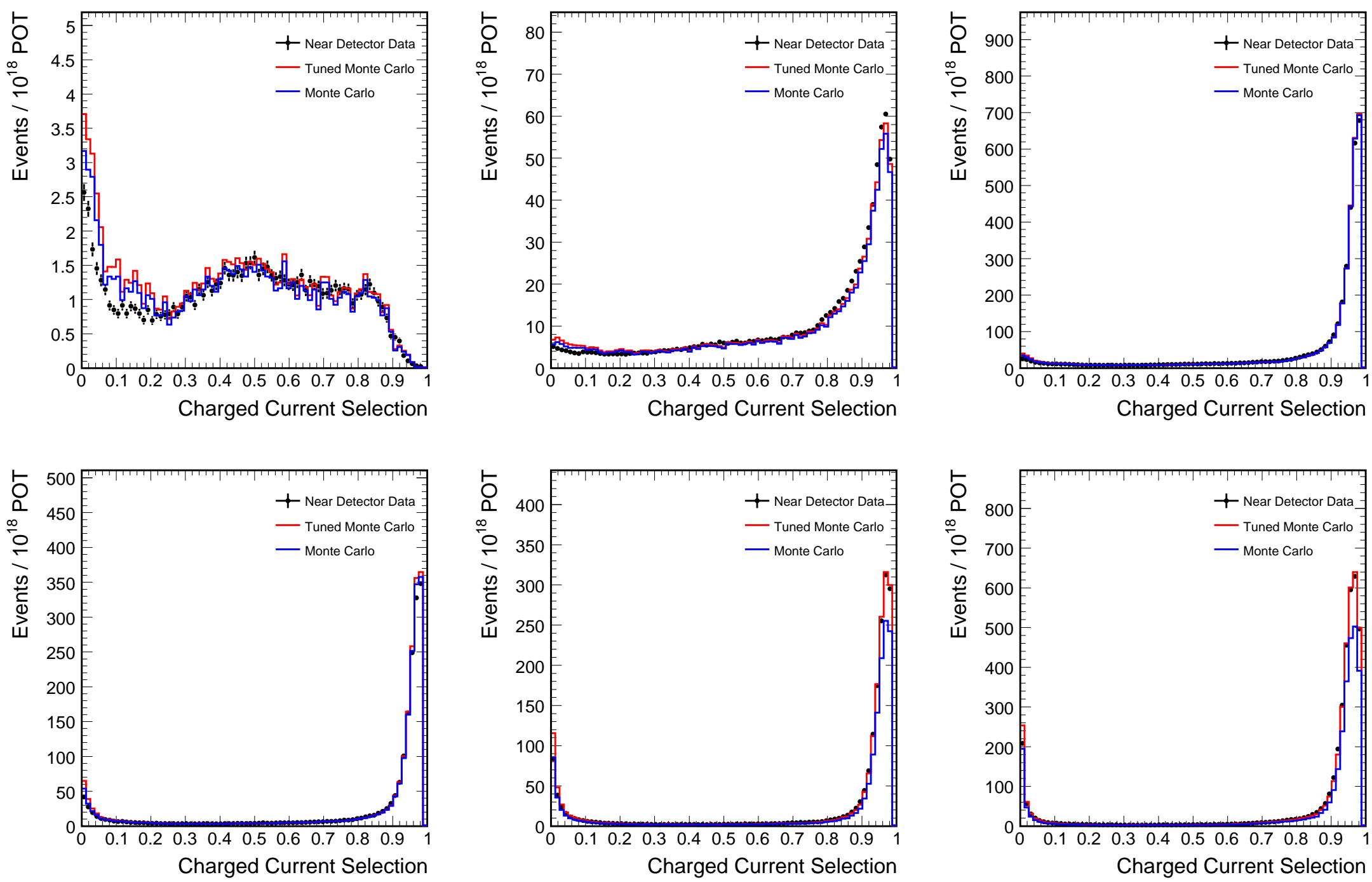

Figure 6.11: The CC discriminator for different neutrino energies for LE RunII data and Monte Carlo. The plots show the following energy ranges 0-1 GeV (top left), 1-2 GeV (top middle), 2-4 GeV (top right), 4-6 GeV (bottom left), 6-10 GeV (bottom middle), $>10 \mathrm{GeV}$ (bottom right). 

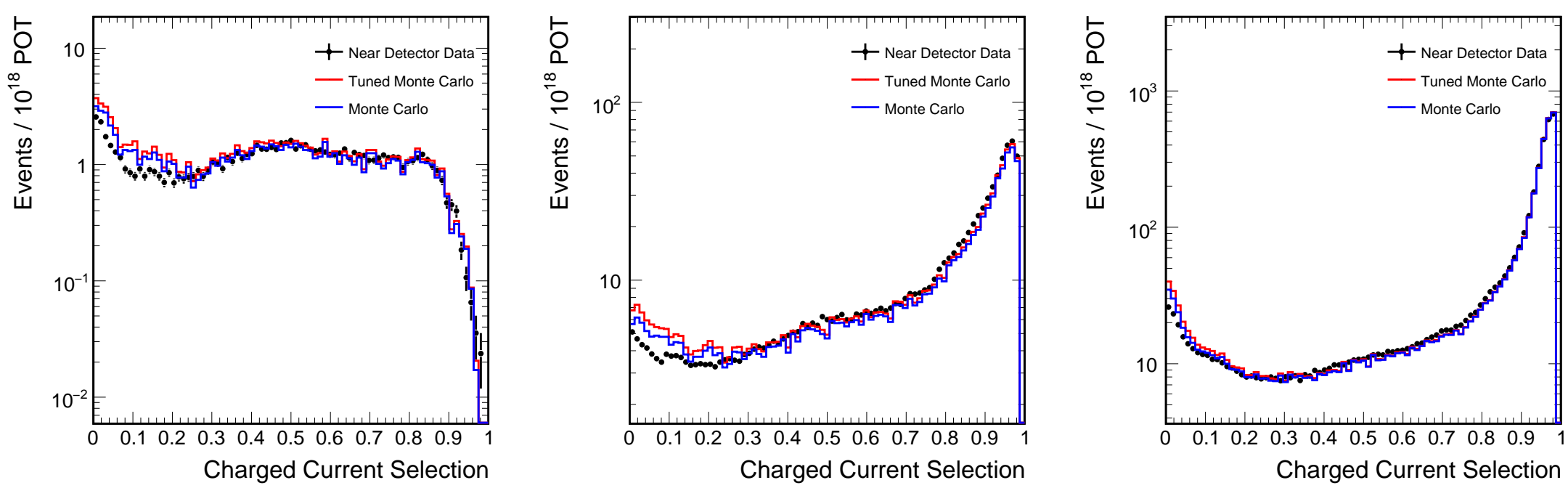

Figure 6.12: The charged current discriminator for different neutrino energies on a log scale for LE RunII data and Monte Carlo The plots show the following energy ranges 0-1 GeV (top left), 1-2 GeV (top middle), 2-4 GeV (top right), 4-6 GeV (bottom left), 6-10 GeV (bottom middle), $>10 \mathrm{GeV}$ (bottom right). 


\subsubsection{HE RunII Data/MC Comparison}

This section compares different distributions in the Near Detector for data and Monte Carlo for the HE RunII comprising Figures 6.13 through 6.18. 

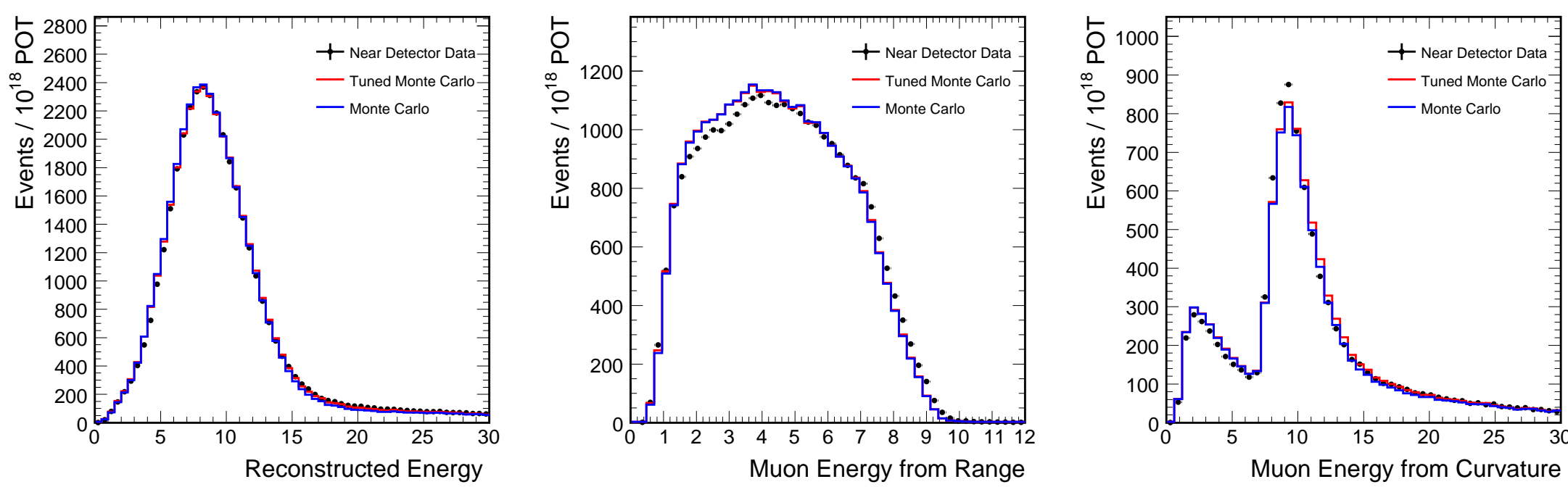

Figure 6.13: Energy plots for the HE RunII data and Monte Carlo. The plots are of neutrino energy (top left), muon energy from range (top middle), muon energy from range (top right), shower energy (bottom left), the number of tracks (bottom middle), the number of showers (bottom right). 

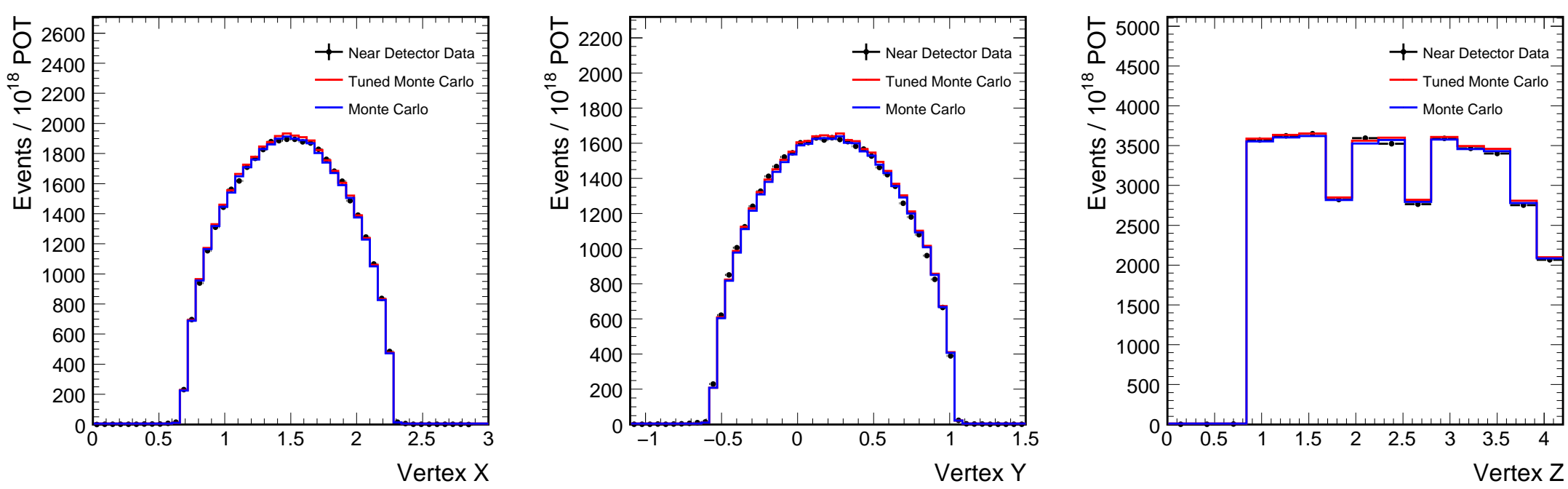

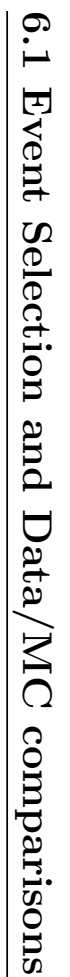
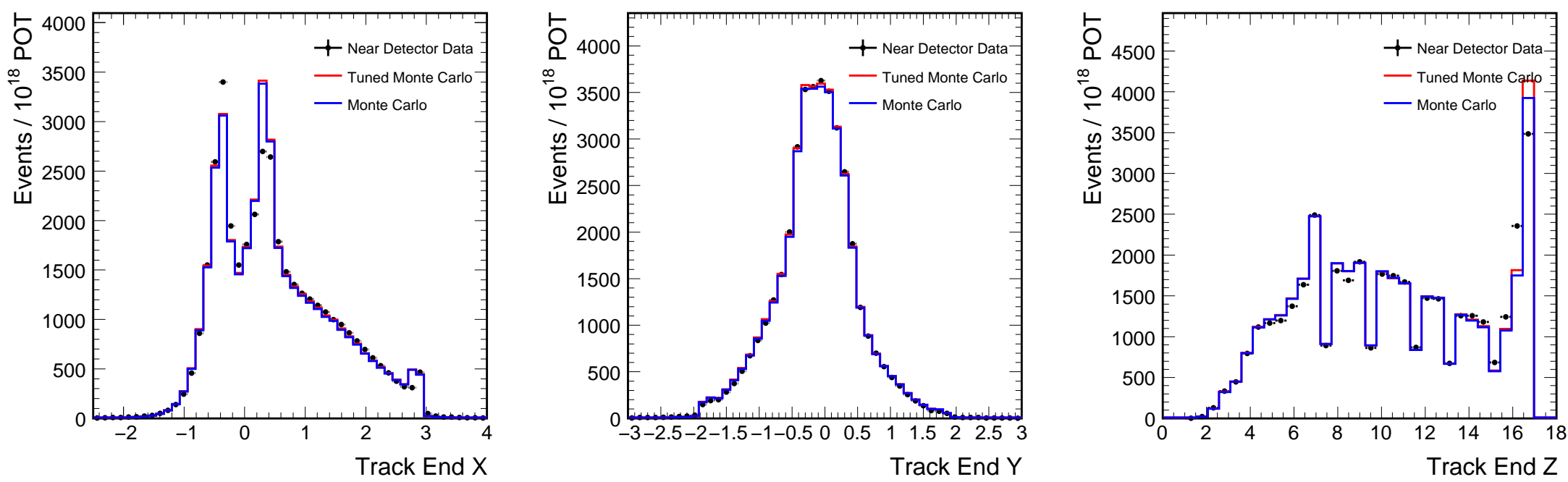

Figure 6.14: Vertex and track end plots for HE RunII data and Monte Carlo. The plots show the position of the event vertex for $x$ (top left), $y$ (top middle), and $z$ (top right), and the track end point for $x$ (bottom left), $y$ (bottom middle), and $z$ (bottom right). 

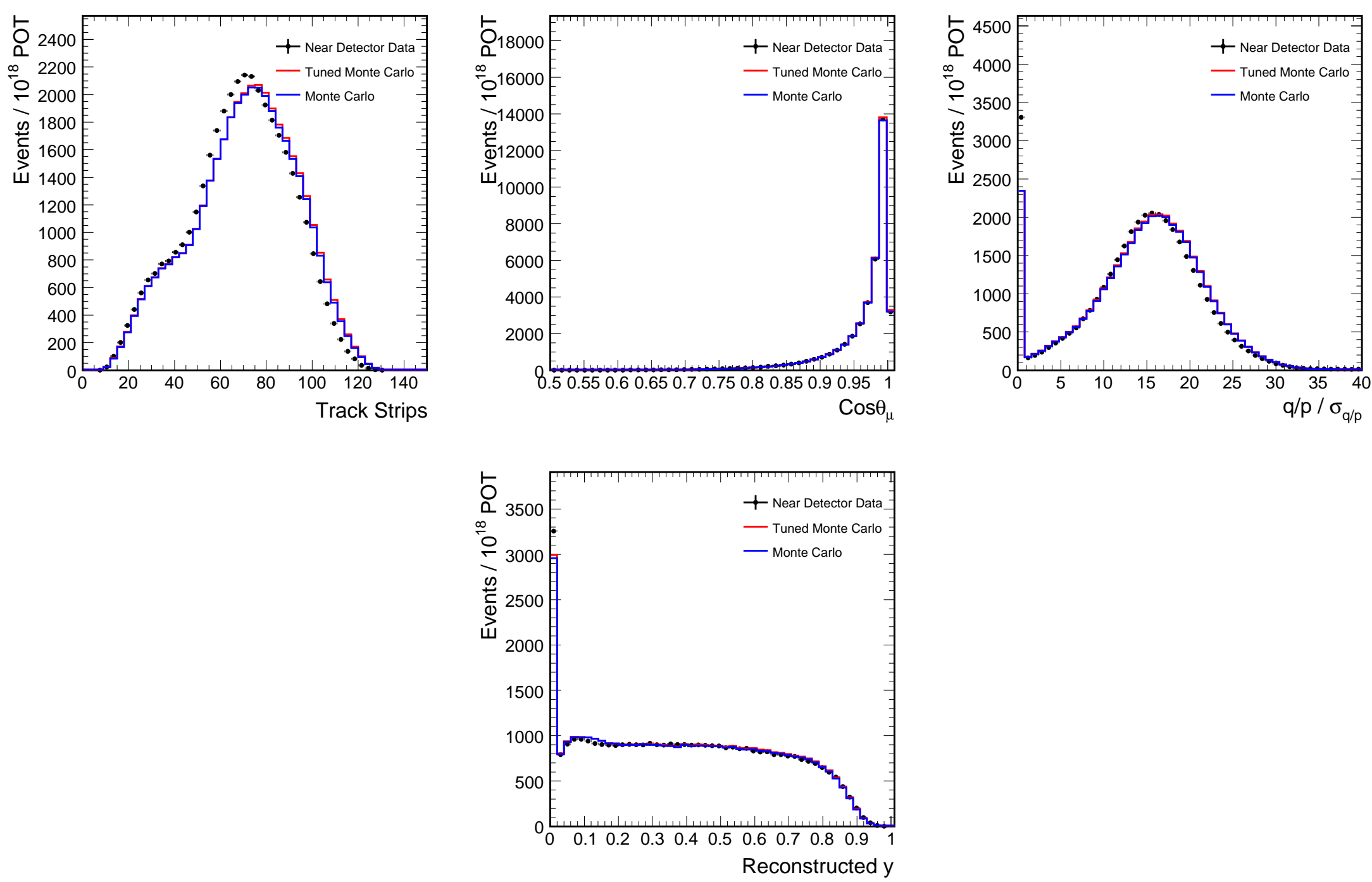

Figure 6.15: Miscellaneous plots for HE RunII data and Monte Carlo. The plots show the number of track strips (top left), the cosine of the angle between the track and beam direction (top middle), $(q / p) / \sigma_{q / p}$ (top right), reconstructed $y$ (bottom). 

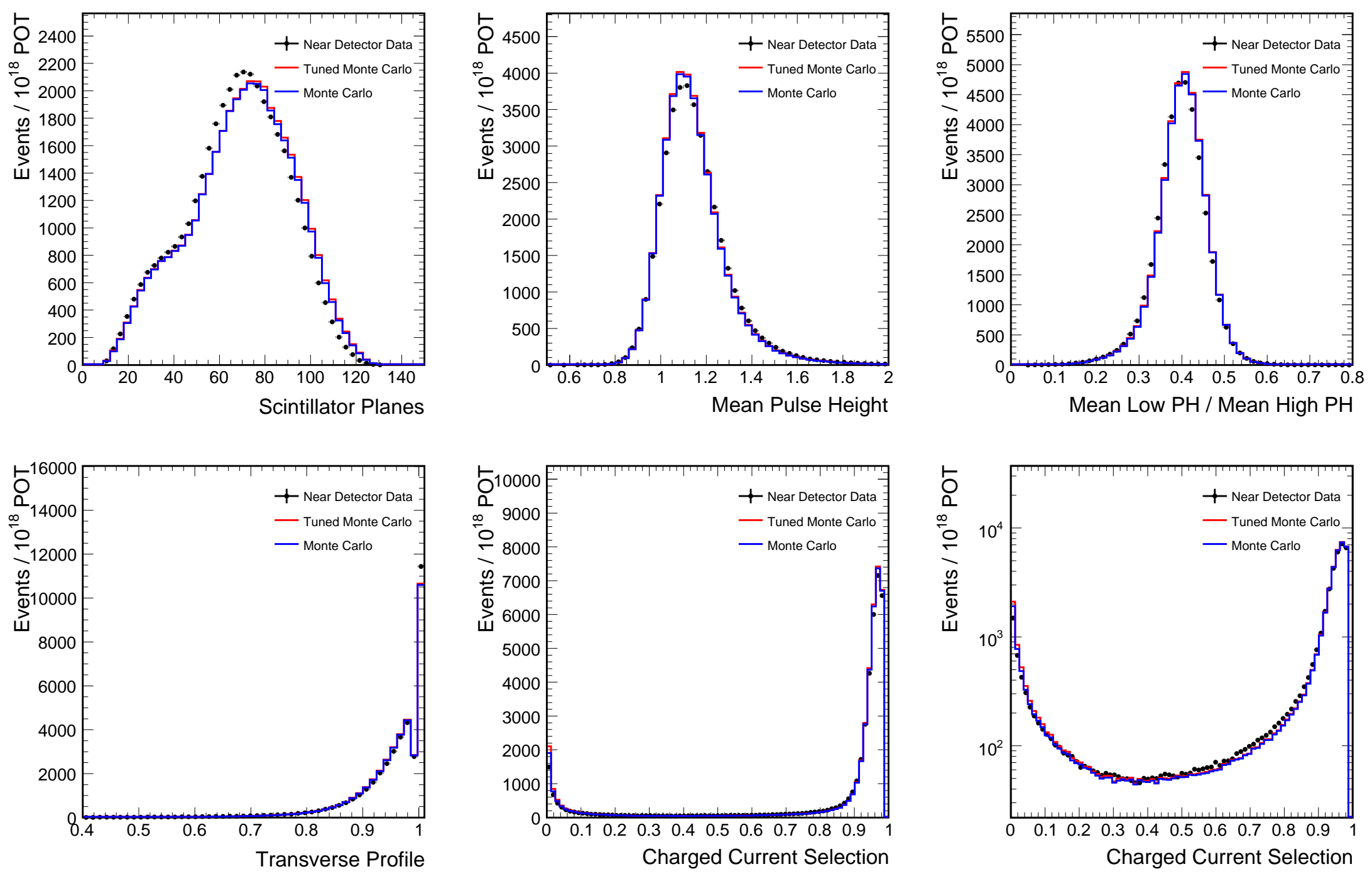

Figure 6.16: CC selection plots for HE RunII data and Monte Carlo. The plots show the number of scintillator planes (top right), the mean pulse height (top middle), low mean pulse height/high mean pulse height (top right), the transverse profile (bottom left), the CC discriminator (bottom middle), the CC discriminator on a log scale (bottom right). 

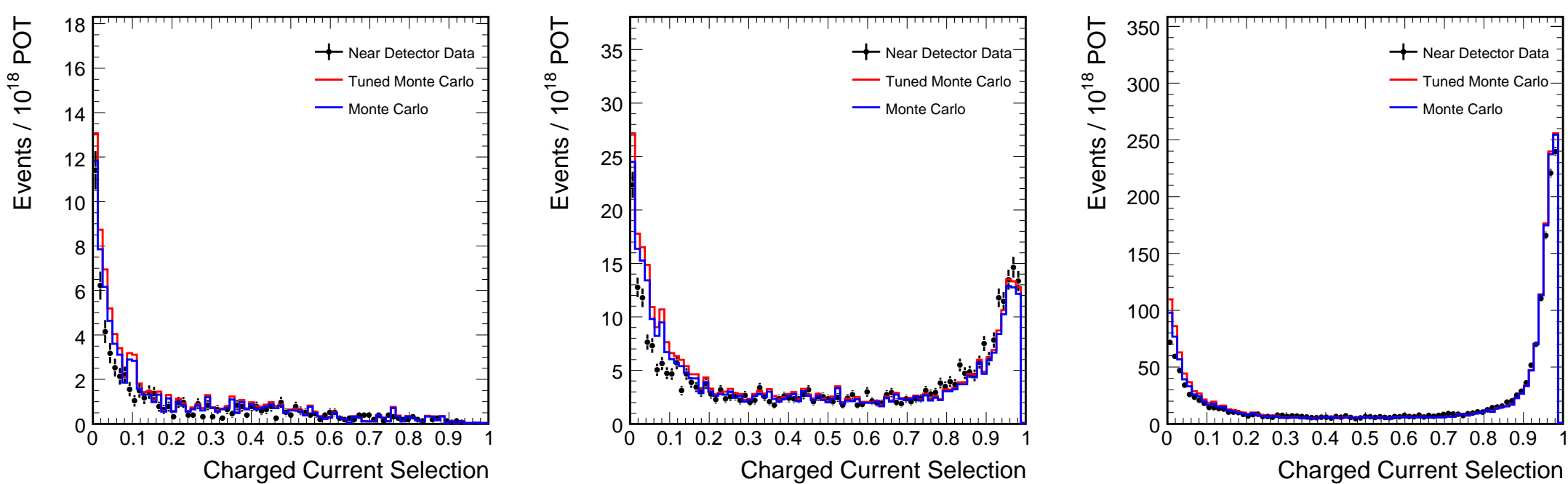

Figure 6.17: The CC discriminator for different neutrino energies for HE RunII data and Monte Carlo. The plots show the following energy ranges 0-1 GeV (top left), 1-2 GeV (top middle), 2-4 GeV (top right), 4-6 GeV (bottom left), 6-10 GeV (bottom middle), $>10 \mathrm{GeV}$ (bottom right). 

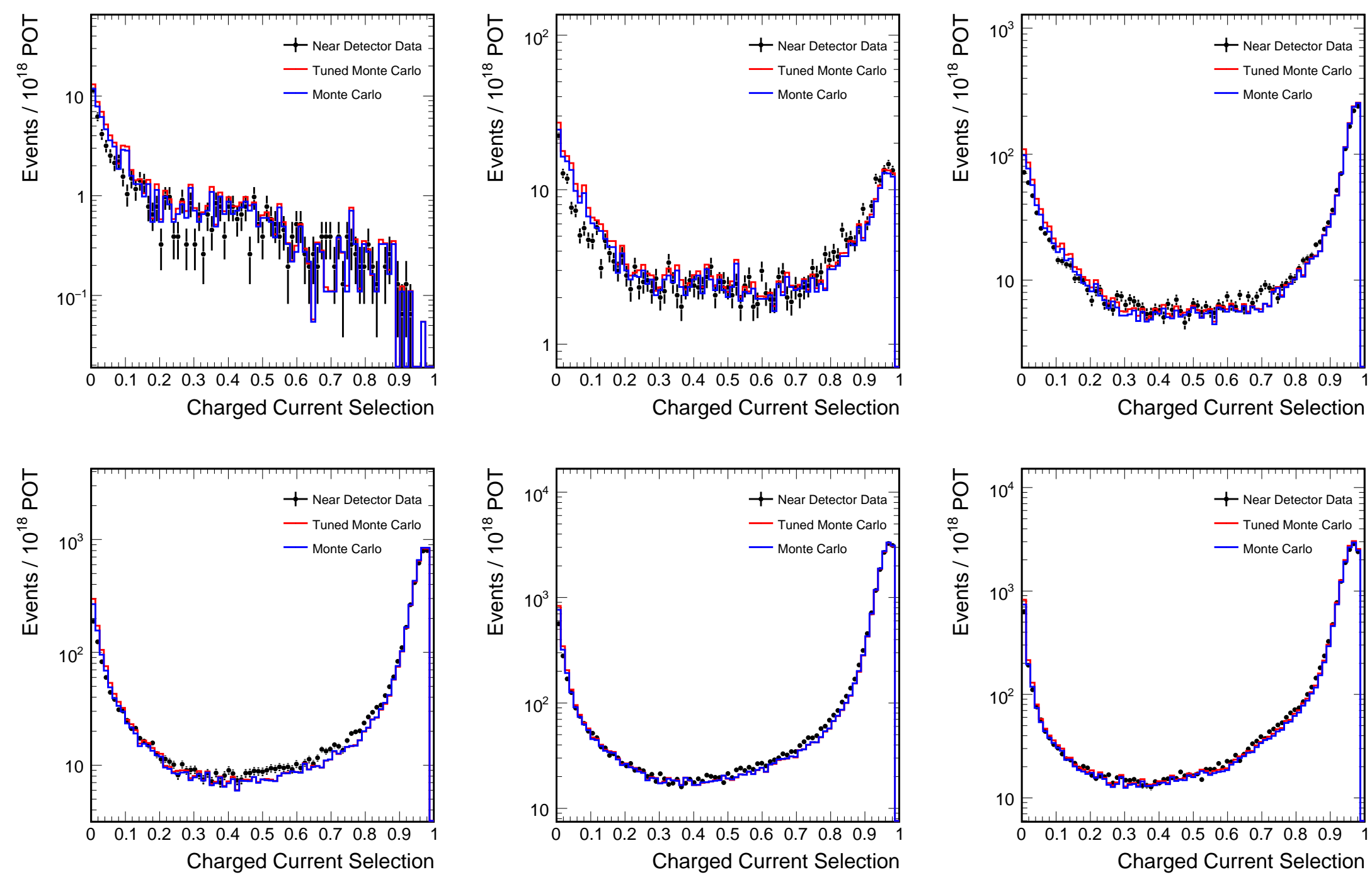

Figure 6.18: The CC discriminator for different neutrino energies on a log scale for HE RunII data and Monte Carlo. The plots show the following energy ranges $0-1 \mathrm{GeV}$ (top left), 1-2 GeV (top middle), 2-4 GeV (top right), 4-6 GeV (bottom left), 6-10 GeV (bottom middle), $>10 \mathrm{GeV}$ (bottom right). 


\subsubsection{Comments}

Overall there is good agreement in the Near Detector after the Monte Carlo tuning has been applied. The weighting did not result in substantial changes to the Monte Carlo distributions for the HE configuration, and therefore agreement between data and Monte Carlo is better in that configuration. Some discrepancies seen, mainly in the LE configuration, are:

i. Events with low y: The tuning does a better job at predicting the number of events with low $y$ than the nominal Monte Carlo, but there remains a problem in the lowest $y$ bin. (see the bottom plots of Figures 6.3, 6.9 and 6.15.)

ii. Muon Track End Position: The track end $x$ does not agree well for those that stop near the center of the detector where the coil is located. (see the bottom left plots of Figures 6.2, 6.8 and 6.14.)

iii. Neutrino Energy: The yield at the peak of the neutrino energy spectrum disagrees at the $5 \%$ level this will be further discussed in Section 8.1.2. (see the bottom left plots of Figures 6.1 and 6.7).

The source of these discrepancies is not fully understood and will require work to make the predictions more accurate. These problems do not pose a significant setback to using the tuning to predict the Monte Carlo in the Far Detector.

\subsection{Extrapolation to the Far Detector}

The extrapolation procedure takes the data from the Near Detector and uses it make an unoscillated prediction at the Far Detector. The fact that the Near Detector receives neutrinos that have decayed at wider angles causes a larger fraction of the flux to arrive from higher energy pions that decayed at the end of the decay pipe. The neutrino energy depends on both the energy of the parent hadron and on the angle of the decay. These 


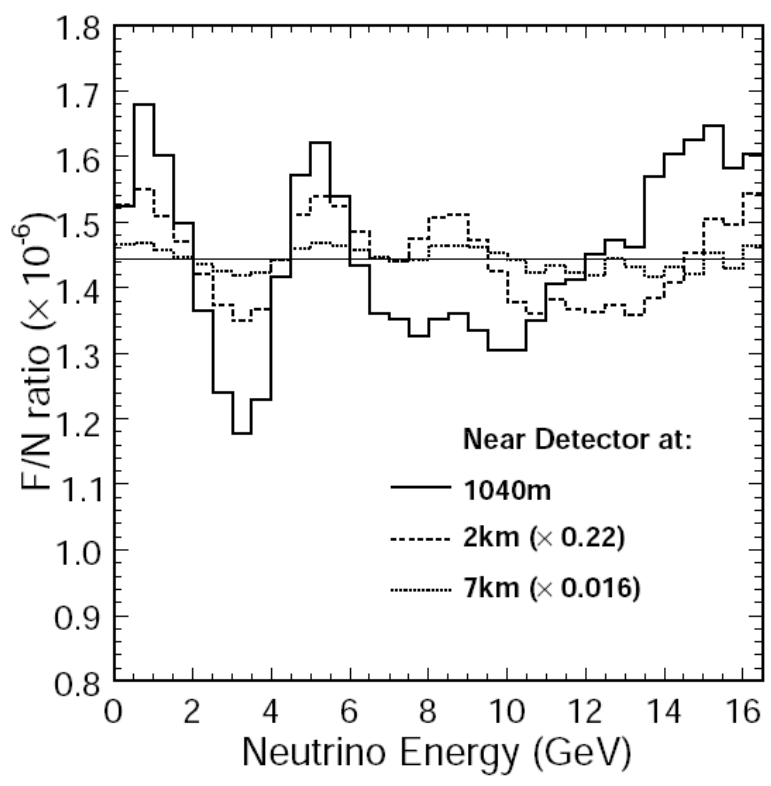

Figure 6.19: The Far/Near ratio for the Near Detector in different locations. The default position is $1040 \mathrm{~m}$ from the target, but moving it back to $7 \mathrm{~km}$ removes much of the structure. Taken from [45].

effects cause differences between the Near and Far Detectors. As the Near Detector is moved away from the neutrino source the differences are removed. Figure 6.19 shows that for a Near Detector located at $7 \mathrm{~km}$ from the target, the differences are less than $2 \%$.

These effects are not difficult to model GNUMI, but there are several approaches on how to use this information to make an extrapolation to the unoscillated Far Detector spectrum. Two different philosophies have been used to make this extrapolation. The first philosophy uses Monte Carlo to produce a transfer function to incorporate Near and Far differences. The simplest example of this would be taken the Near Detector and multiply each bin by the Far/Near ratio as found in the Monte Carlo. The second philosophy uses the Near Detector data to correct and constrain the Monte Carlo. The tuned Monte Carlo Monte Carlo can then be used predict the Far Detector spectrum. Both approaches are complimentary and are sensitive to different sources of systematic error. 


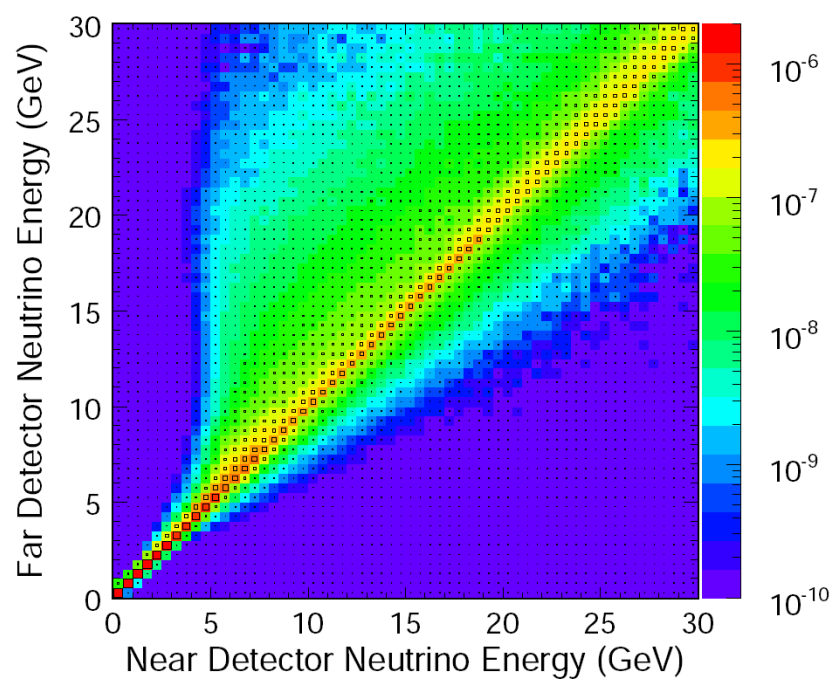

Figure 6.20: A matrix of energies in the Far Detector compared to the Near Detector. Each bin is the mean number of $\nu_{\mu}$ events in the Far Detector for one event in the Near Detector. Taken from [46].

The Beam Matrix method [77] was chosen as the primary analysis extrapolation method for MINOS. From the Monte Carlo, it creates a matrix that can be directly applied to the Near Detector data. The matrix relates the neutrino energy of events observed in the Near Detector to the distribution of events in the Far Detector. It incorporates knowledge of the geometry of the beamline, the focusing elements, two body decay kinematics and the different solid angles seen by the detectors. The matrix is shown in Figure 6.20

We have seen that the tuning of the Monte Carlo done in Chapter 5 provides a good agreement between data and Monte Carlo in the Near Detector. This same set of tuning parameters can used to modify the Far Detector Monte Carlo to predict the unoscillated spectrum. This is the extrapolation method chosen for this thesis. It provides some advantages over the more direct methods discussed in that it uses information available from many different beam configurations. However, the amount of time needed to do a full fit to the Near Detector is much longer. 

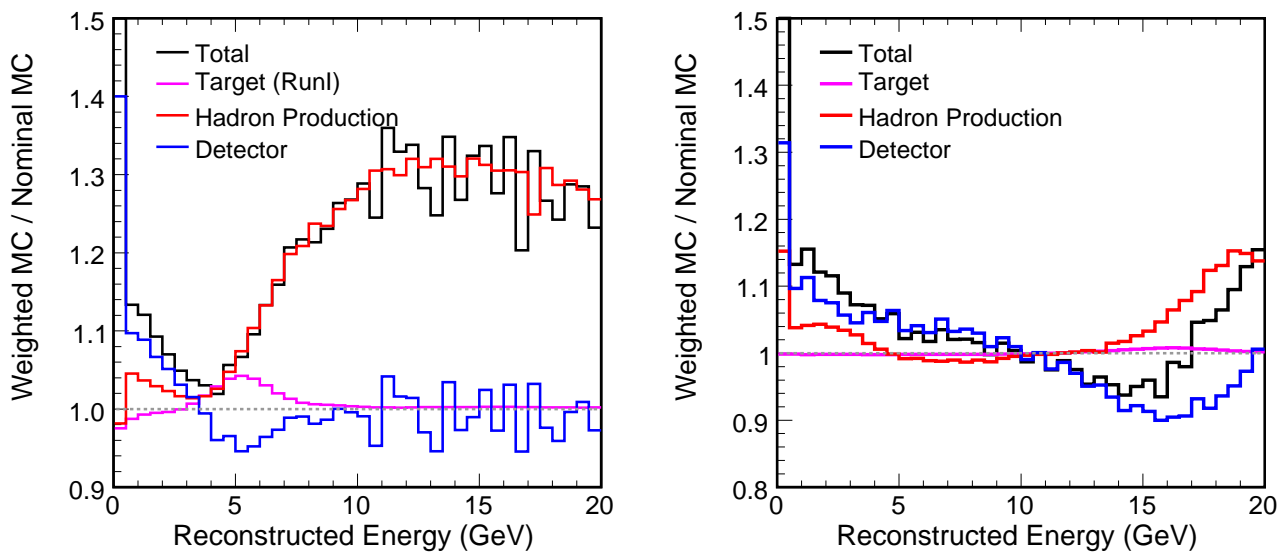

Figure 6.21: Relative contributions from the different weighting components for the LE (left) and HE (right) beam configurations. The black is the total weight, the purple is from the target position, the red is from hadron production, and the blue from detector parameters.

\subsubsection{Reweighting}

Figure 6.21 separates the reweighting of the Far Detector Monte Carlo into different components for the beam, hadron production and detector parameters. RunI and RunII have slightly different weightings because of the position of the target. 


\section{Chapter 7}

\section{Selecting Charged Current Events}

\subsection{Charged Current Events}

Since all neutrino flavors interact via the Neutral Current (NC) interaction (within the Standard Model picture of three light, active neutrinos) oscillations will not change the number of observed NC events in the Far Detector. The neutrino flavor cannot be determined in such an interaction, since a hadronic shower, if that, is the only remnant in the detector. On the other hand, Charged Current (CC) events, produce a lepton in the final state which allows the flavor of the neutrino to be inferred. The ability to tag the resulting lepton is the key to identifying $\mathrm{CC}$ events and the resulting oscillation parameters.

There have been two general strategies used to distinguish $\mathrm{NC}$ from $\mathrm{CC}$ events. The first focuses only on the track of a particular neutrino event and tries to measure the muon-ness of that track. NC events will also produce tracks from hadrons, but these should have significant differences from muon tracks. The second approach tries to use all the information from the event including information from the shower in attempting to make the distinction. Three different selection methods will be described and contrasted. 


\section{$7.2 \quad \mathrm{kNN}$ Method}

One method for selecting CC events, referred to as the kNN Method [78] and developed by Rustem Ospanov, focuses only on information from the track in the event. NC events with tracks should exhibit strong differences when compared to those formed by muons. It uses four different variables to distinguish these differences:

i. Number of Scintillator Planes - Tracks found in NC events will typically be shorter than from a CC event.

ii. Mean Pulse Height - The average energy deposited per hit is different between muons and other particles. Minimum ionizing particles such as muons deposit the same average energy. A hadronic track will deposit on average more energy per hit.

iii. Signal Fluctuation - This variable is constructed by sorting the track hits in order of ascending pulse height. The hits are then divided into two samples the low and high pulse height hits. The variable is defined as the ratio of the mean of the low pulse height hits to the mean of the high pulse height hits. There should be little variation between the low and high pulse height hits for a muon.

iv. Transverse Profile - The pulse height of all detector hits that fall within a 4 strip window and a 37.36 ns time window is compared to the pulse height of the track. The variable is defined as the ratio of these two numbers. A muon track should have few stray hits around the track and will have typically one hit per plane.

For the last three variables, the first part of the track near the hadronic shower was excluded to enhance the sensitivity to the differences between muon and non-muon tracks. Figure 7.1 compares each of these different variables for muon and non-muon tracks.

A k-nearest neighbor $(\mathrm{kNN})$ algorithm was used to classify the events using these four variables. This algorithms is evaluated in a multi-dimensional space, one for each variable. The probability that an event is $\mathrm{CC}$ or $\mathrm{NC}$ is found by drawing a sphere around the event in this parameter space. 

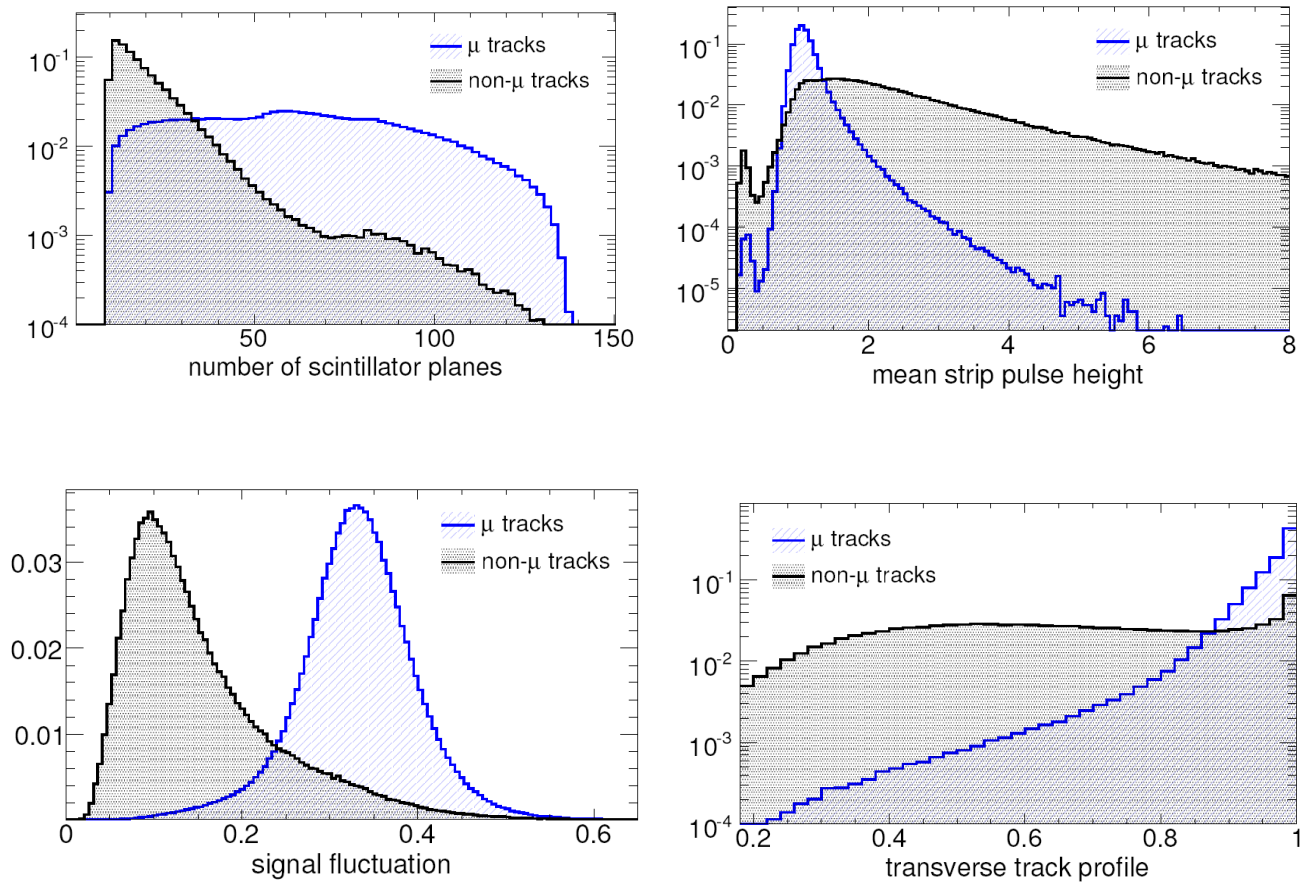

Figure 7.1: The four different separation variables used in the standard algorithm to select CC events. Top Left: the number of scintillator planes. Top Right: the average pulse height deposited in a strip. Bottom Left: The fluctuation in the average pulse height deposited. Bottom Right: Extra hits in and around the selected the track found. Taken from [78]. 
Using a predefined Monte Carlo training sample, events are counted within the multidimensional sphere. An event in a region populated preferentially by true CC events is more likely to be a $\mathrm{CC}$ event.

As implemented, this algorithm requires events to have at 5 hits in each view. Since reconstructed tracks are required to have at least 2 planes in each view, a small fraction of tracks found by the reconstruction will not be assigned a kNN output. Studies have shown that these events are dominantly NC events and therefor are discarded from the analysis. One feature of this method is that because the emphasis is on the track of the event, the training algorithm has been shown to be robust enough for data in any beam configuration.

\subsection{Likelihood or PDF Method}

The likelihood method [79], developed by Andy Blake, uses one and two dimensional probability density functions(PDFs) to characterize the differences between charged and NC events. The following variables were used to create four PDFs (see Figures 7.2 and 7.3):

i. number of track-like planes

ii. the average pulse height per track-like plane

iii. $|q / p| / \sigma_{q / p}$ vs track length, where $q$, and $p$ are the charge and momentum determined by the track fitter and $\sigma_{q / p}$ is the error.

iv. reconstructed $y$

v. the reconstructed charge of the muon

vi. the track length 
The total probability of a particular event being a $\mathrm{CC}$ or $\mathrm{NC}$ event is calculated by multiplying the probability for each PDF:

$$
\begin{aligned}
& P_{C C}=P(C C) \prod_{i} P\left(x_{i} \mid C C\right) \\
& P_{N C}=P(N C) \prod_{i} P\left(x_{i} \mid N C\right),
\end{aligned}
$$

where $P(C C)$, and $P(N C)$ are the relative normalization between $\mathrm{CC}$ and $\mathrm{NC}$ events and $P\left(x_{i} \mid C C\right)$, and $P\left(x_{i} \mid N C\right)$ are the probability of obtaining an event whose $i^{\text {th }}$ variable has a given $x$ value. The discriminating variable is constructed by:

$$
\text { Parameter }=\frac{P_{C C}}{P_{C C}+P_{N C}}
$$

This method ignores any possible correlations between different separation variables except those explicitly constructed in the two dimensional PDFs. This method was largely an extension of the selection algorithm used in the first published MINOS results [80]. An earlier version of this method, using only four PDFs was chosen as the official method for results published in the summer of 2007 [81].

\subsection{ANN Method}

The ANN [82] method, developed by Niki Saoulidou, uses an artificial neural network to define a figure of merit to separate CC events. All events with more than 50 planes are automatically identified as $\mathrm{CC}$ events allowing the training algorithm to focus in on the key characteristics differing between the two event types. The variables chosen as inputs to the neural network are:

1. event length

2. total event pulse height

3. number of strips in event 

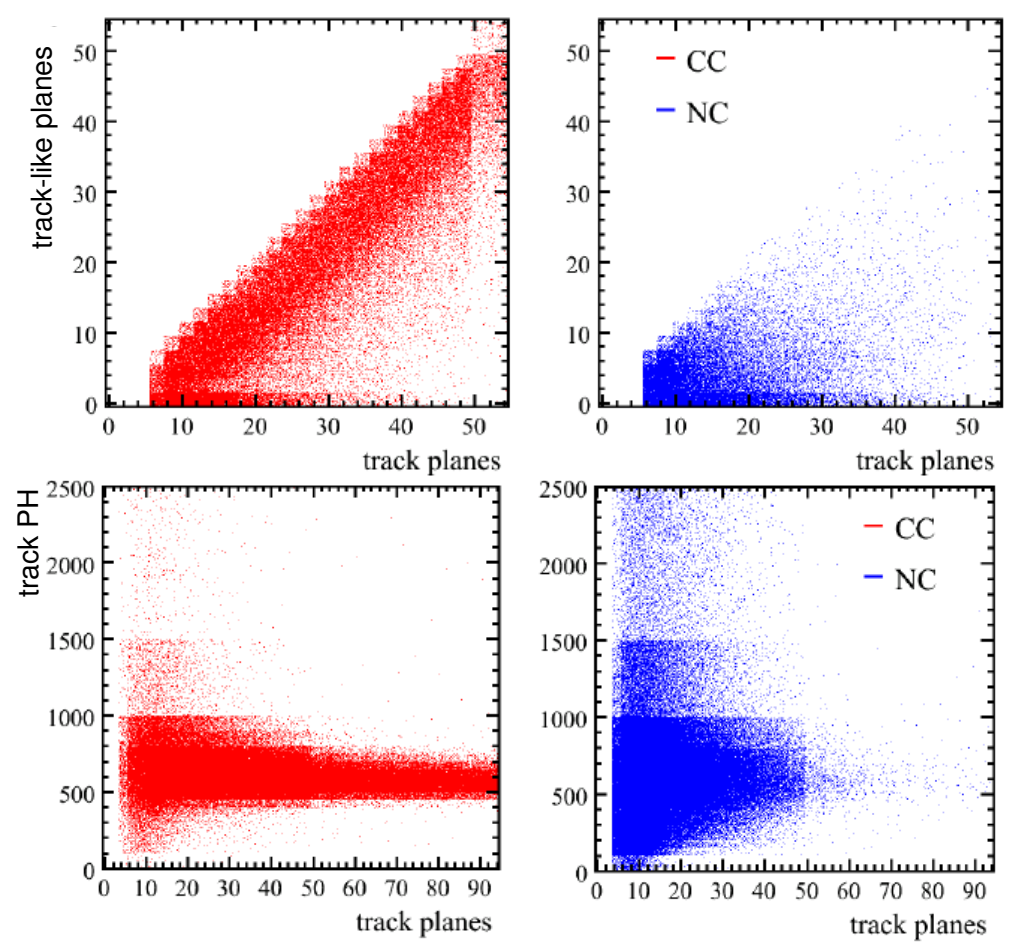

Figure 7.2: The top two plots show the number of track-like planes as a function of the number of track planes for charged current (left), and $\mathrm{NC}$ (right) events. The bottom two show the track pulse height as function of track planes and for $\mathrm{CC}$ (left), and NC (right) events. Taken from [79] 

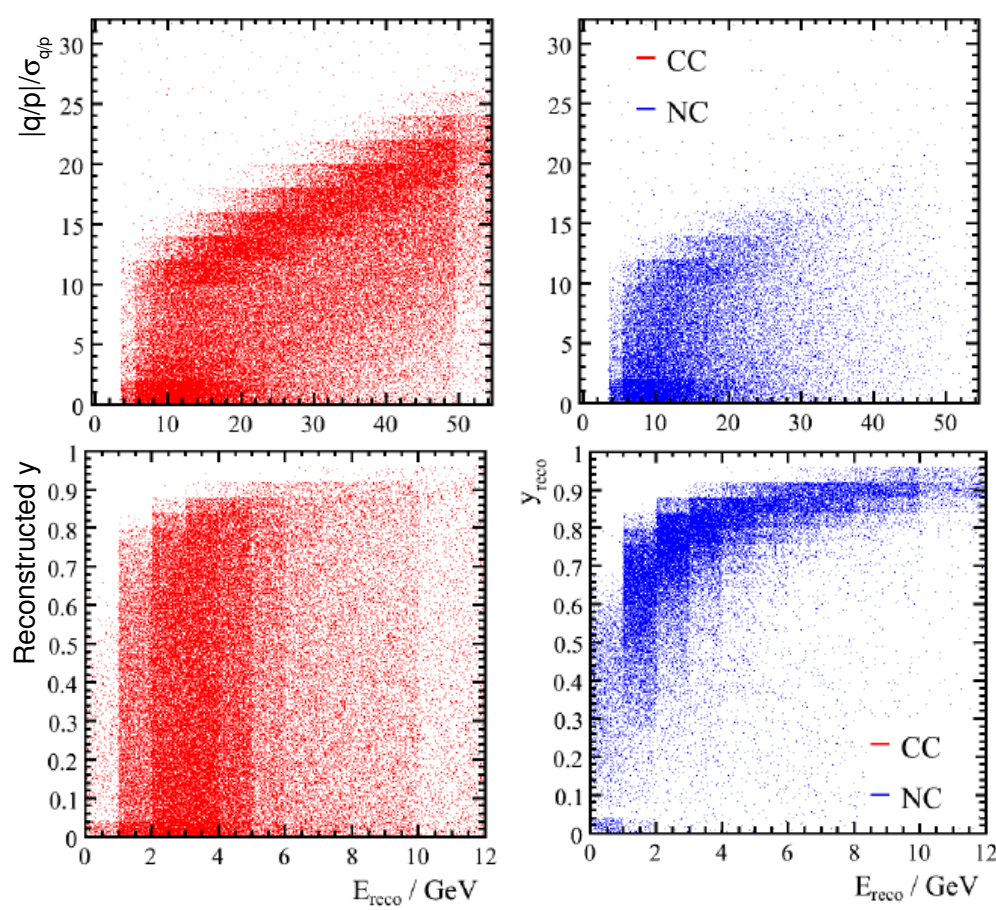

Figure 7.3: The top two plots show $|q / p| / \sigma_{q / p r}$ as a function of the number of neutrino energy for charged current (left), and NC (right) events. The bottom two show the reconstructed $y$ as a function of neutrino energy and for CC (left), and NC (right) events. Taken from [79]. 
4. number of tracks in the event

5. the average pulse height per plane in the event

6. the average pulse height per strip in the event

7. the fraction of pulse height in the first 3 planes of the event

8. the fraction of pulse height in planes 3-6 of the event

9. the fraction of pulse height in the last 6 planes of the event

10. the average pulse height per plane in the track

11. the average pulse height per digit in the shower

12. the difference between the track and shower lengths in the $U$ view

13. the difference between the track and shower lengths in the $V$ view

These variables are shown in Figures 7.4 and 7.5. 

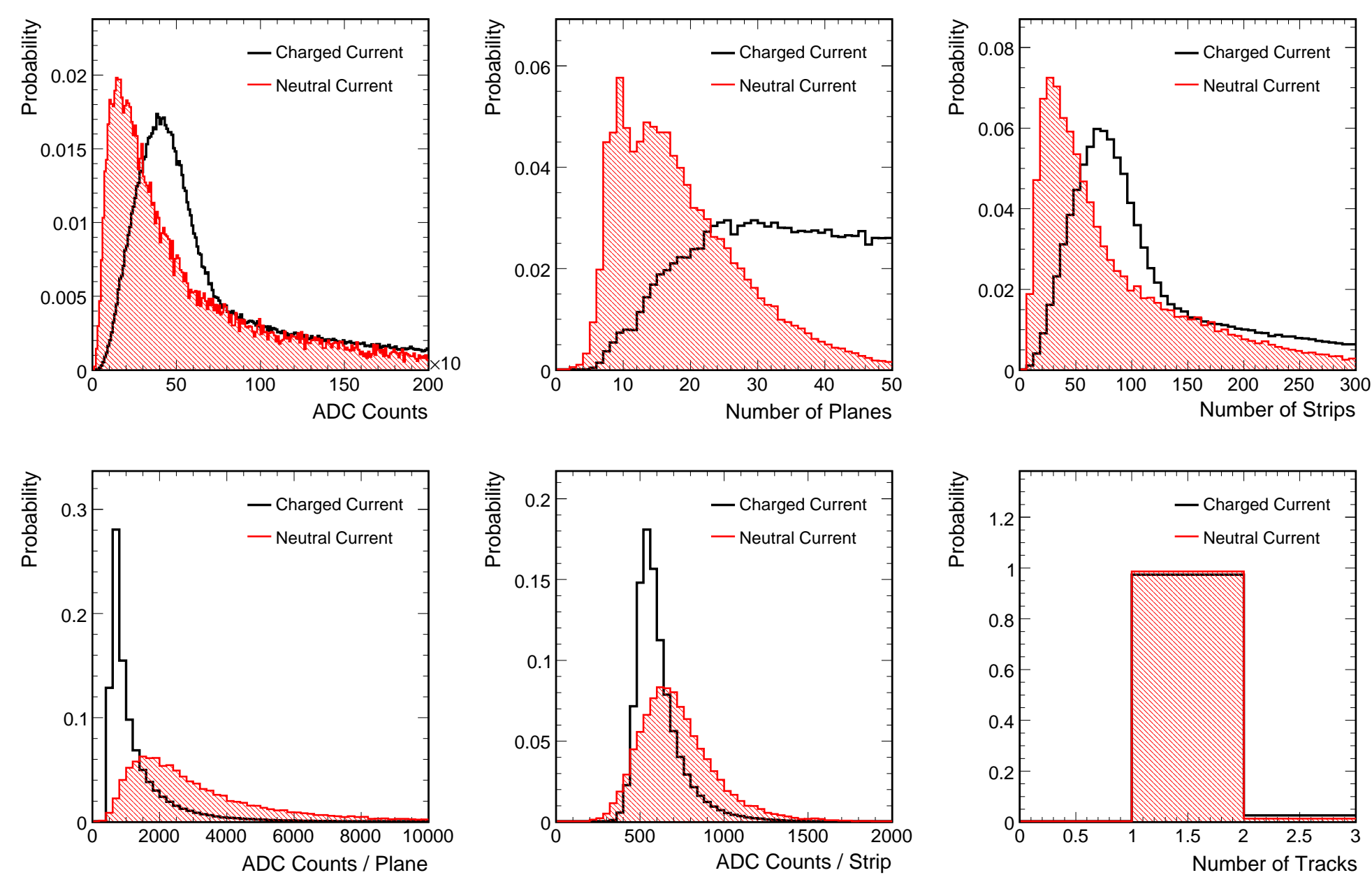

Figure 7.4: The ANN input variables: event pulse height (top left), event length( top middle), event strips (top right), event pulse height per plane ( bottom left), event pulse height per strip (bottom middle), the number of tracks (bottom right). The red histograms are for neutral current and black for CC. 

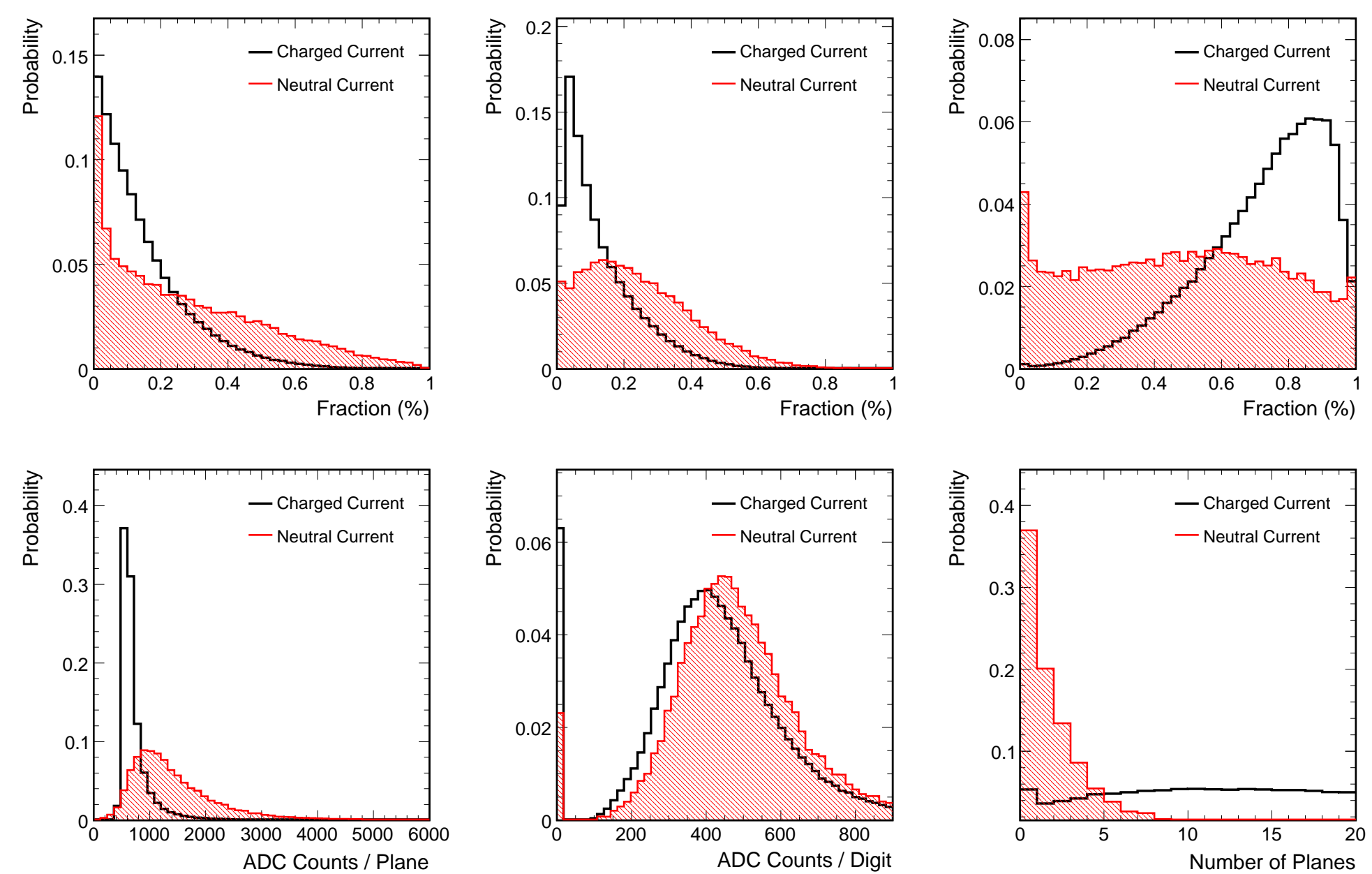

Figure 7.5: The ANN input variables: fraction of pulse event pulse height in the first three planes (top left), from planes three to six (top middle), the last six planes (top right), the track pulse height per plane (bottom left), the shower pulse height per digit (bottom middle), and the difference between the length of the track and shower. The red histograms are for NC and black for CC. 


\subsection{A Comparison of Selection Algorithms}

Some general observations between the different methods are:

i. The $\mathrm{kNN}$ algorithm, in contrast to the other methods, uses only information on the reconstructed track. This simplifies the training process for different neutrino beam configurations, but may not be taking advantage of all information in an event.

ii. The ANN and PDF algorithms execute more rapidly because they do not have to loop through an entire training set to calculate the output. Execution time may become a limiting problem for a kNN algorithm with a large number of variables.

iii. The ANN and PDF algorithms can easily handle additional variables, while the $\mathrm{kNN}$ cannot practically use many more than four or five for the execution time considerations mentioned above. This isn't necessarily an advantage since many variables are correlated.

iv. The ANN and kNN algorithms fully incorporate correlations between the different selection variables, while the PDF algorithm only uses two-dimensional correlations between selected variables.

v. The variables used in the $\mathrm{kNN}$ algorithm are more complicated than the corresponding variables in the ANN and PDF algorithms. This may lead to a higher dependence on the detailed modeling of detector response in the Monte Carlo simulation.

vi. The output of the $\mathrm{kNN}$ algorithm is given in discrete values between 0 and 1 while the other two methods provide continuous discriminant variables.

An important factor in any classification algorithm is how well the selection variables agree between data and Monte Carlo. Several studies [83] have shown that all three methods perform equally well. The one exception is the signal fluctuation variable for the $\mathrm{kNN}$ algorithm. A clear shift has been seen with respect to data and Monte Carlo. If a cut is made excluding low pulse height hits much of this discrepancy is mitigated. The 


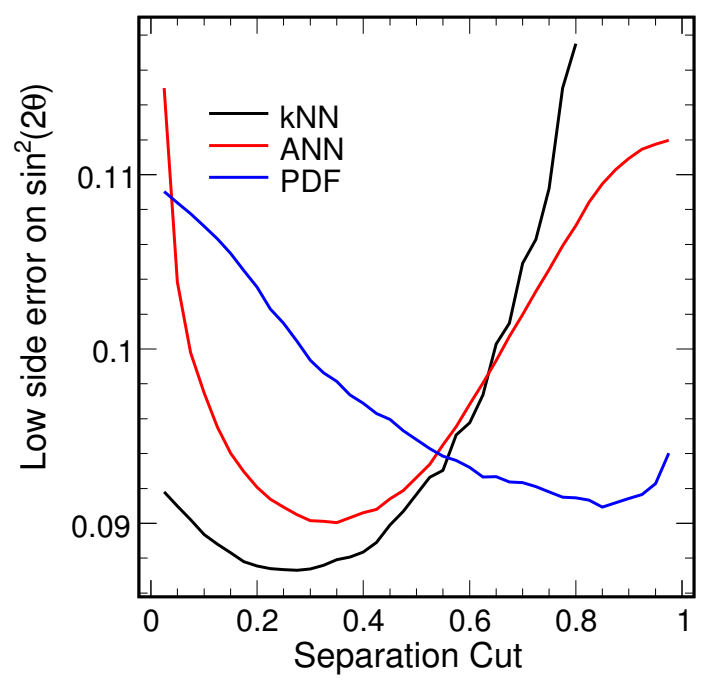

Figure 7.6: Comparison of the sensitivity on the low side $1 \sigma$ error on $\sin ^{2}\left(2 \theta_{32}\right)$ of different $\mathrm{CC}$ algorithms. For the kNN and PDF algorithms, more CC-like events are found to the right, and to the left for the ANN algorithm. The kNN, ANN, and PDF are in black, red and blue respectively.

physical origin of this problem is not fully understood at this time. Thus, selection on the basis of which discriminator best matches the data does not favor one above another..

Perhaps the most important consideration as to the choosing of a discrimination variable is how well it is able to constrain the oscillation parameters: $\Delta m_{32}^{2}$ and $\sin ^{2}\left(2 \theta_{32}\right)$. Using the full set of Far Detector Monte Carlo, each algorithm was tested to see its ability to constrain the oscillation parameters. For these studies a subset of the Far Detector Monte Carlo sample was scaled to $3.21 \times 10^{20}$ POT (the amount of data in the LE configuration). This subset was used as "fake" data oscillated with values of $\sin ^{2}\left(2 \theta_{32}\right)=1.0$ and $\Delta m_{32}^{2}=2.46 \times 10^{-3} \mathrm{eV}^{2}$. For each cut point on the output of the three algorithms, the Monte Carlo was fit to the fake data and the error on the oscillation parameters was found. The error on $\Delta m_{32}^{2}$ was calculated as the average of the high and low $1 \sigma$ errors. For $\sin ^{2}\left(2 \theta_{32}\right)$, the error was evaluated as the low $1 \sigma$ error due to the presence of the physical boundary. Figures 7.6 and 7.7 show the sensitivities for the three selection algorithms. 


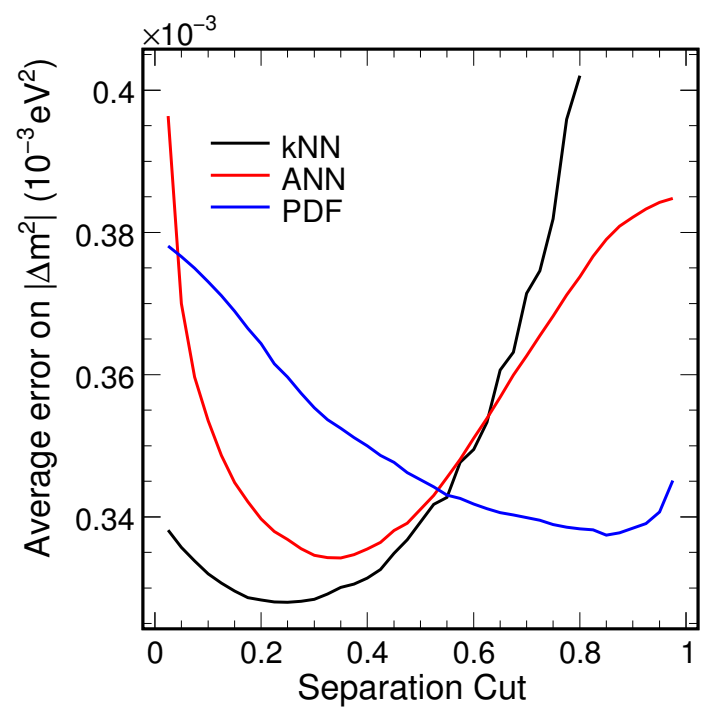

Figure 7.7: Comparison of the sensitivity on the average $1 \sigma$ error on $\Delta m_{32}^{2}$ of different $\mathrm{CC}$ algorithms. For the $\mathrm{kNN}$ and PDF algorithms, more CC-like events are found to the right, and to the left for the ANN algorithm. The kNN, ANN, and PDF are in black, red and blue respectively.

From these plots the optimal cut point for the methods are found to be greater than 0.275 for $\mathrm{kNN}$, less than 0.35 for ANN, and greater than 0.85 for PDF. The kNN method clearly has $2-3 \%$ better sensitivity than both of the other methods. As a result, the $\mathrm{kNN}$ method was selected by the MINOS collaboration as the official CC selection algorithm. Events with values above 0.3 were labeled as $\mathrm{CC}$ events. The purity and efficiency as a function of energy for the kNN method are shown in Figure 7.8.

To investigate whether the main advantage of the kNN method over the others was in the variables used or in the $\mathrm{kNN}$ algorithm itself, a test was done using the same $\mathrm{kNN}$ variables but a different algorithm. The $\mathrm{kNN}$ variables were implemented into a simple neural network using the TMVA toolkit [84]. Figure 7.9 compares the sensitivity to the mixing angle for both methods (the effect on $\Delta m_{32}^{2}$ is the same). The plot shows a neural network trained with the $\mathrm{kNN}$ variables is able to perform equally as well. 


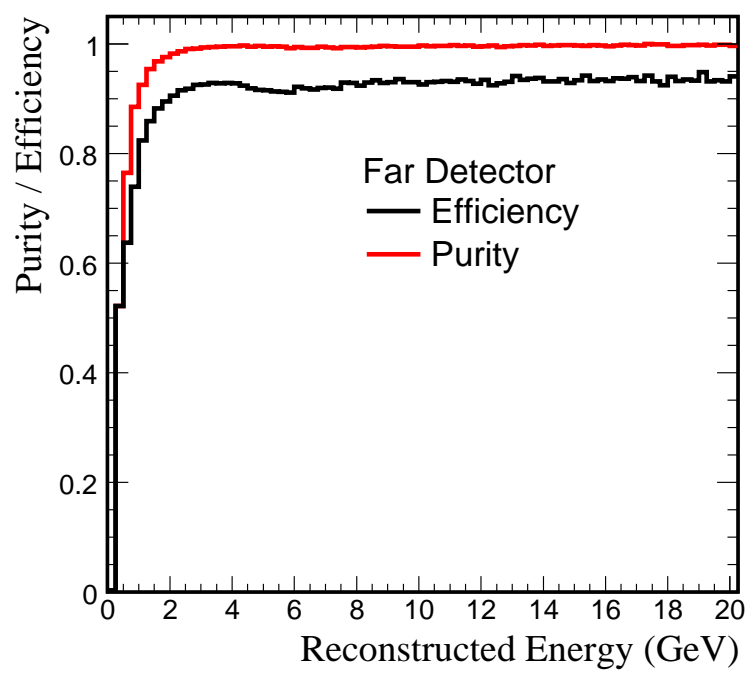

Figure 7.8: The efficiency (black) and purity (red) of the kNN selection algorithm after all the pre-selection cuts for events in the Far Detector as a function of reconstructed neutrino energy.

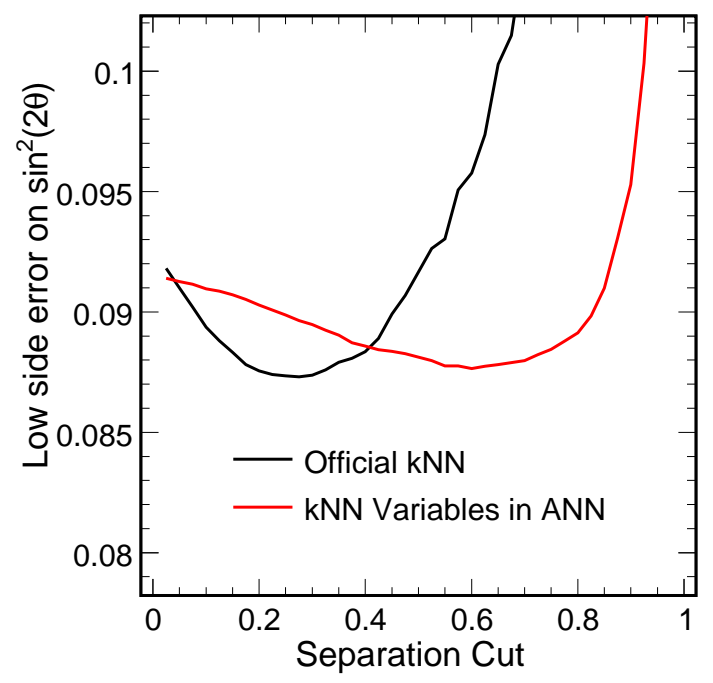

Figure 7.9: Comparison of the sensitivity on the low side $1 \sigma$ error of $\sin ^{2}\left(2 \theta_{32}\right)$ for the standard kNN selection (black) and a neural network using the same variables (red). 


\subsection{Improvements Over the Standard Implementa- tion}

Referring to Figure 7.8 there appears to be further areas of improvement in the low energy region. A hand scan [85] from the Far Detector data also identified several CC events that were missed by the kNN algorithm. Several different areas of improvement are possible. Some of these may include:

i. Remove the 5 plane cut requirement in each view. Figure 7.10 shows a comparison of different neural network algorithms based on the kNN variables, but trained with different minimum requirements on the number of planes. Only a very small improvement was seen by lowering the minimum plane number to 3 .

ii. Additional variables that have been suggested include:

(a) the amount of energy lost at the end of a track. A hadron track will lose a large amount of energy at the end of the track leaving a clear signature.

(b) the length a track extends outside of the hadronic shower

(c) look for gaps in tracks, hadron tracks are much more likely to contain gaps

These have been implemented in a neural network as additional variables to the $\mathrm{kNN}$ variables and only minor improvements were seen.

iii. Use an energy dependent cut instead of a global CC cut.

iv. Provide separate training samples for high and low energy events, similar to what is done in the ANN method. 


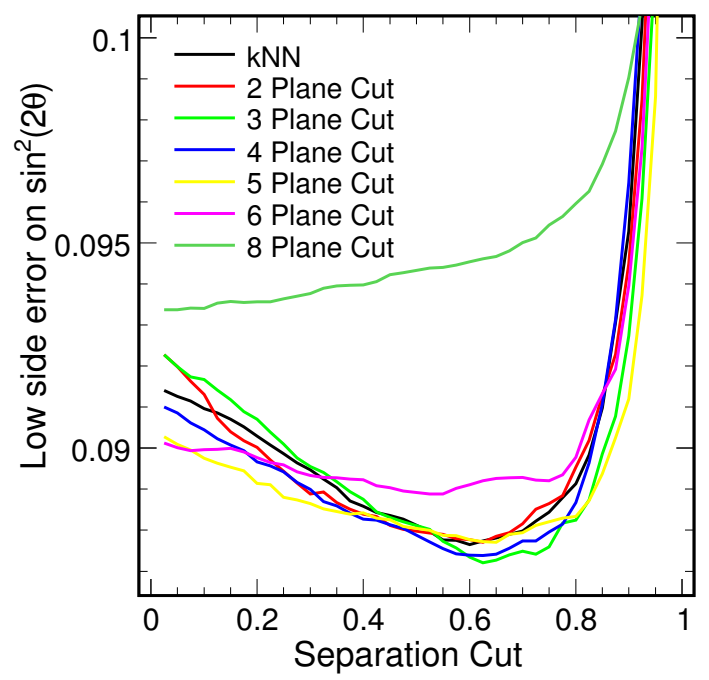

Figure 7.10: Comparison of the sensitivity on the low side $1 \sigma$ error of $\sin ^{2}\left(2 \theta_{32}\right)$ for different requirements on the minimum number of tracks in each plane. 


\section{Chapter 8}

\section{Systematic Uncertainties}

Systematic errors can potentially affect the values of the measured oscillation parameters. This chapter will describe the sources of systematic error that are most important for the atmospheric oscillation analysis. The errors and their associated systematic uncertainties are evaluated through a combination of studies using data and Monte Carlo samples.

\subsection{Beam Related Uncertainties}

Most of the neutrino beam uncertainties were studied in the context of the work reported in Chapter 5. This section discusses two sources of beam related systematic error that were not considered in the fit to the Near Detector data. These are the effect of the geomagnetic field, and the variation seen at low energies in the Near Detector as a function of time during the running periods relevant for this analysis.

\subsubsection{Geomagnetic Field in the Decay Pipe}

The MINOS beam simulation does not account for the effect of the geomagnetic field on hadrons as they travel the length of the $675 \mathrm{~m}$ decay pipe. Calculations done early on in MINOS, suggested [86] that the geomagnetic field may have a non-negligible effect on the neutrino beam. The author of those calculations urged for a complete Monte 


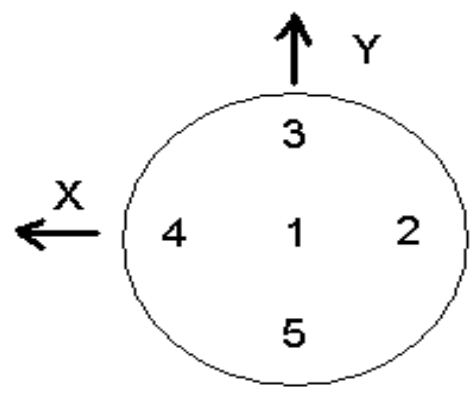

Figure 8.1: The coordinate system used to measure the geomagnetic field; $x$ is SouthWest or beam-left and $y$ is vertical. The field was measured five times at each $z$ location graph in the order indicated on the graph. The measurement in each quadrant was done one foot from the 78 in. diameter pipe wall. Figure courtesy Jim Hylen.

Carlo simulation to fully understand its impact, but it was never carried out. More recently the CNGS beam saw an effect in their muon monitors that may be explained by the geomagnetic field [86]. This section describes the results of incorporating actual measured values of the magnetic field in the decay pipe into the GNUMI Monte Carlo simulation.

\section{Measurements of the Geomagnetic Field}

The geomagnetic field in the decay pipe was measured by Jim Hylen and others in October 2003 [87]. Measurements were made in five different points along the decay pipe. Figure 8.1 defines the coordinate system used and the five measurement points. The components of the geomagnetic field averaged over the length of the decay pipe are shown in Figure 8.2. The measurements show a uniform field $0.3 \mathrm{G}$ field oriented downward with an uncertainty of $\pm 0.2 \mathrm{G}$. This value is half that of the earth's magnetic field at the surface with the reduction due to partial shielding from the steel. These values were incorporated into the GNUMI simulation along with the beam direction relative to the earth. 


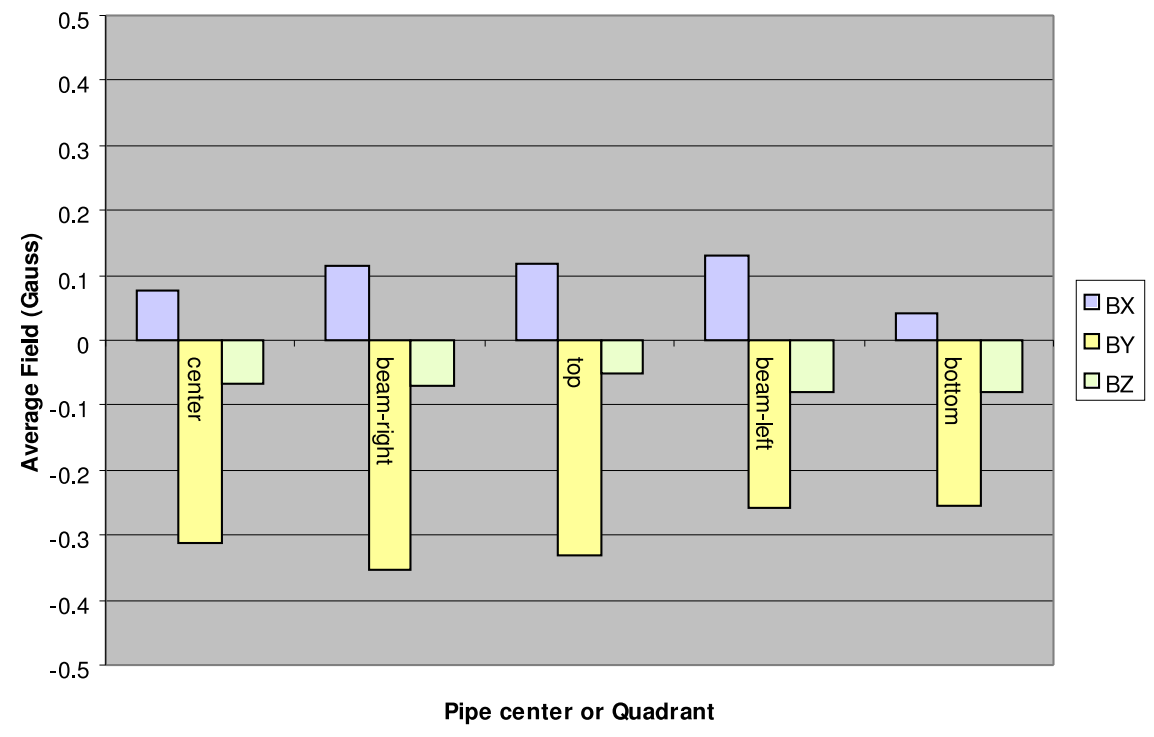

Figure 8.2: The three components of the earth's magnetic field indicated by $B_{x}$ in blue, $B_{y}$ in yellow, $B_{z}$ in light green. The measurements were averaged over the length of the decay pipe for the five different measurement positions (see Figure 8.1). Figure courtesy Jim Hylen.

\section{The Effect of the Geomagnetic field to the NuMI Flux}

Figures 8.3 and 8.4 show a calculation of the flux at different points in the $x, y$ plane or transverse plane (where $z$ is the direction along the beamline). The center of each plot corresponds to the position of the detector. The flux at each point is calculated by summing the probability that individual neutrinos could be detected at that particular point. The probabilities for all the grid points are then normalized to the maximum probability. The plots show that the geomagnetic field moves the neutrino beam downward and to the right $(\sim 10 \mathrm{~cm}$ in the Near Detector, $\sim 100 \mathrm{~m}$ in the Far Detector).

Figure 8.5 shows the effect of the geomagnetic field on on the neutrino energy spectrum. To distinguish between statistical fluctuations and real physics, a simulation with a magnetic field 10 times larger was carried out and then scaled down by a factor of 10 . The change in the fluxes is less than one percent for the Far, Near and Far/Near ratio. Because of the size of this effect it was not included as a systematic error in the oscillation analysis. 

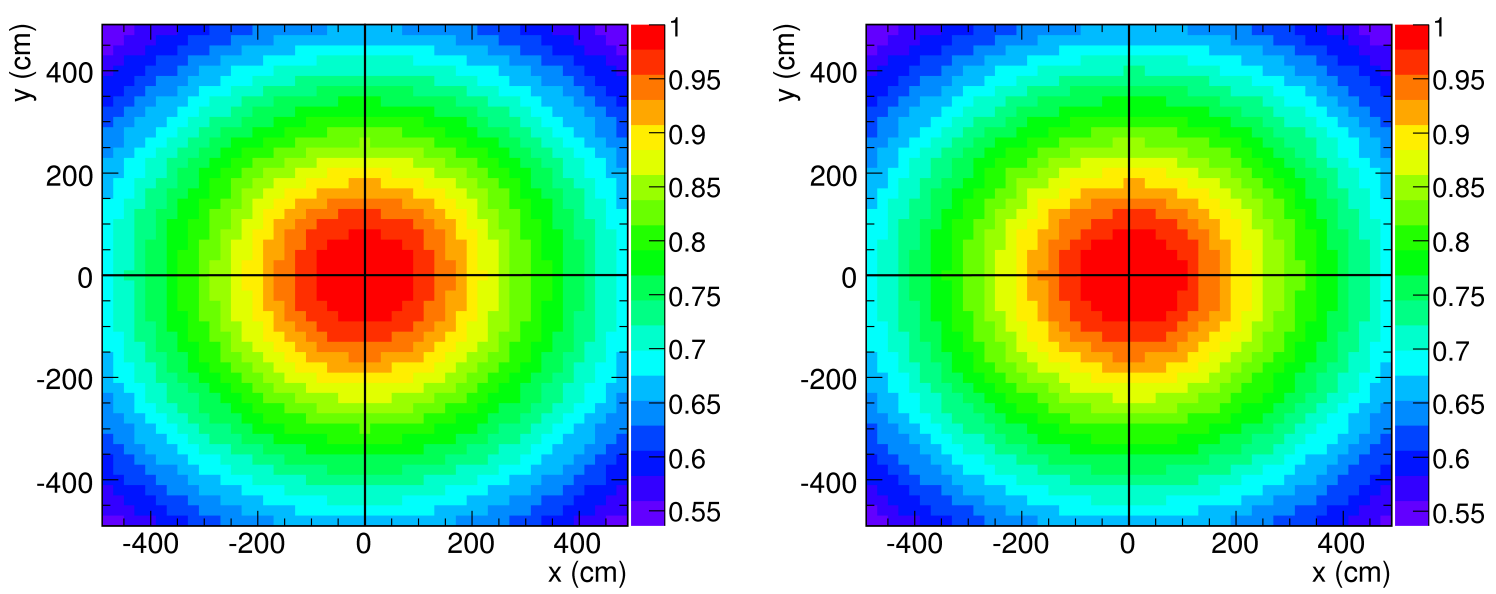

Figure 8.3: A calculation of the relative $\nu_{\mu}$ flux in the plane transverse to the beam in the Near Detector. The graph is centered on the Near Detector and each graph point represents the summed probability that all the generated neutrinos will be detected at that point. The nominal Monte Carlo is on left and the right shows the effect of adding the geomagnetic field to the Monte Carlo.
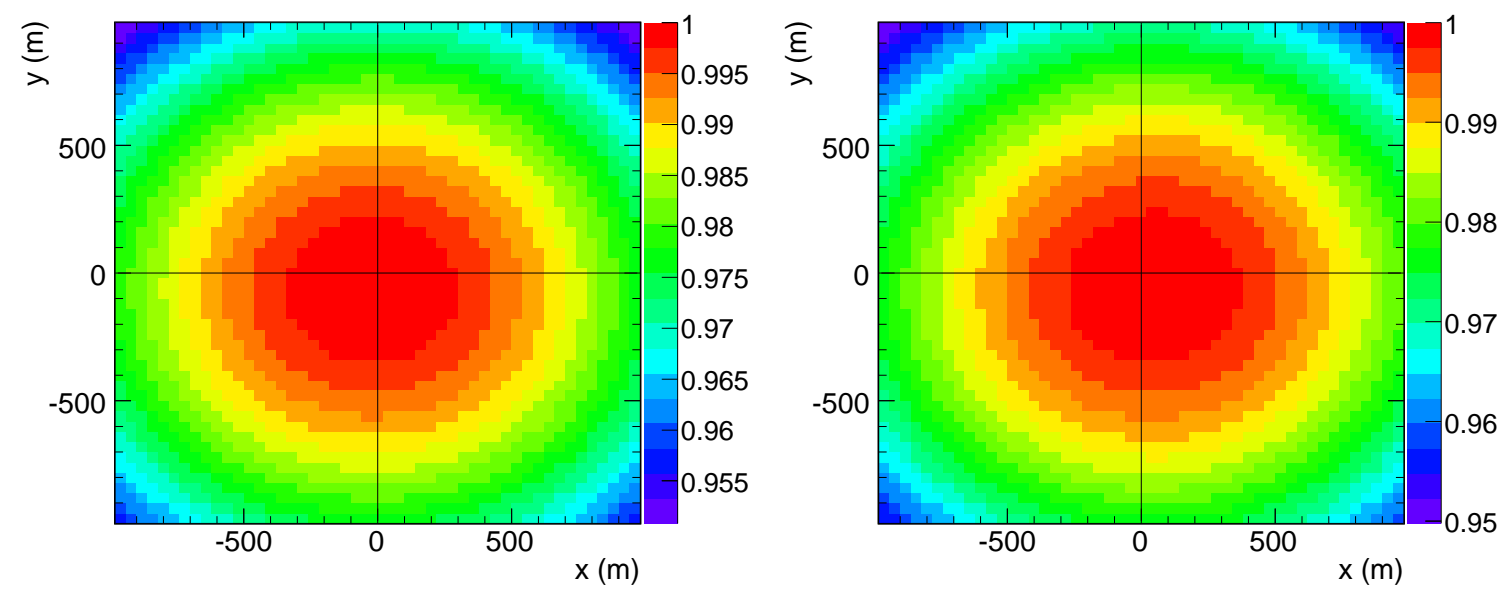

Figure 8.4: A calculation of the relative $\nu_{\mu}$ flux in the plane transverse to the beam in the Far Detector. The graph is centered on the Far Detector and each graph point represents the summed probability that all the generated neutrinos will be detected at that point. The nominal Monte Carlo is on left and the right shows the effect of adding the geomagnetic field to the Monte Carlo. 

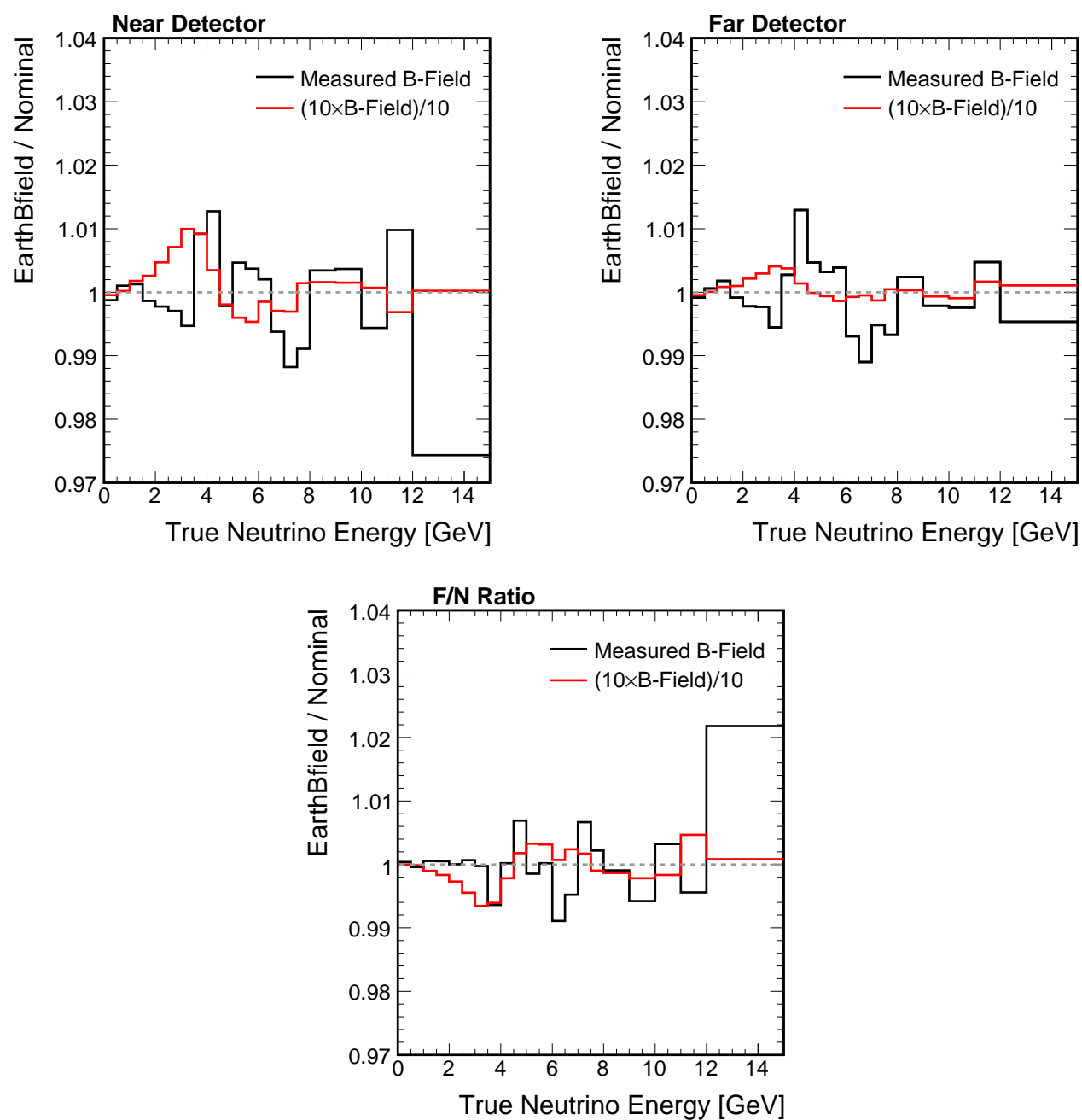

Figure 8.5: The ratio of the nominal neutrino energy spectrum to one with a geomagnetic field for the Near Detector (top right), Far Detector(top left), and the Far/Near ratio (bottom). The black line is the result for the measured magnetic field and the red is a magnetic field $\times 10$ larger and then scaled down by 10 . 

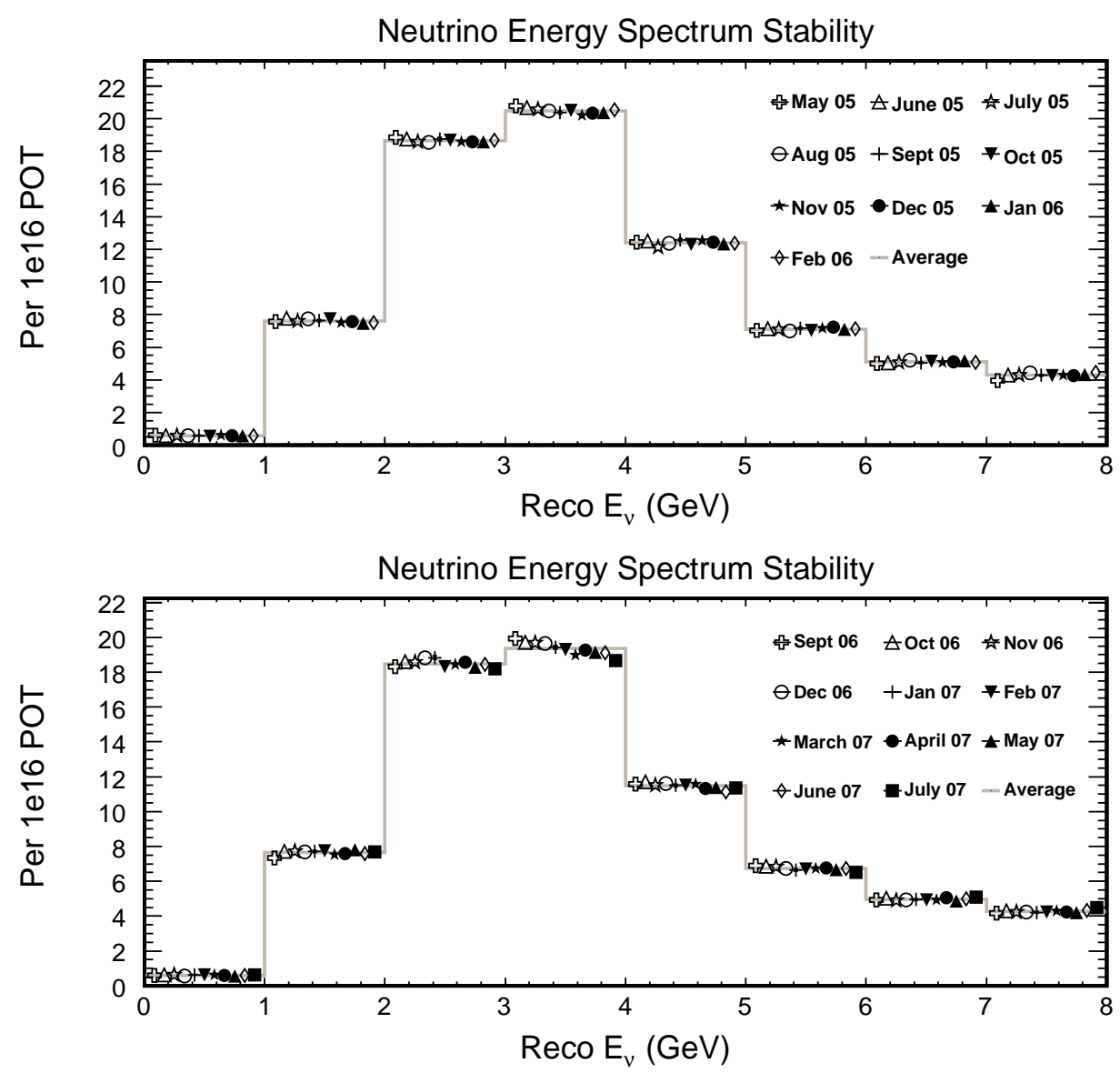

Figure 8.6: The number of neutrino events over time as a function of energy. The top plot is from RunI and the bottom from RunII. Figure courtesy Mark Dorman.

\subsubsection{Time Dependent Change to the Flux}

Over the course of RunI the neutrino energy spectrum was constant over all reconstructed energies. RunII saw a steady decrease in the number of $\nu_{\mu}$ events between 2 and $4 \mathrm{GeV}$ as a function of time. Figure 8.6 compares the neutrino energy spectrum for the different run periods as measured in the Near Detector. During the same time no change was seen to the number of $\bar{\nu}_{\mu}$ events [88].

\section{Possible Sources}

Focusing uncertainties is one example of an effect that would change the number of $\nu_{\mu}$ events but not the $\bar{\nu}_{\mu}$. The largest of these uncertainties are as listed in Section 3.1.6. 

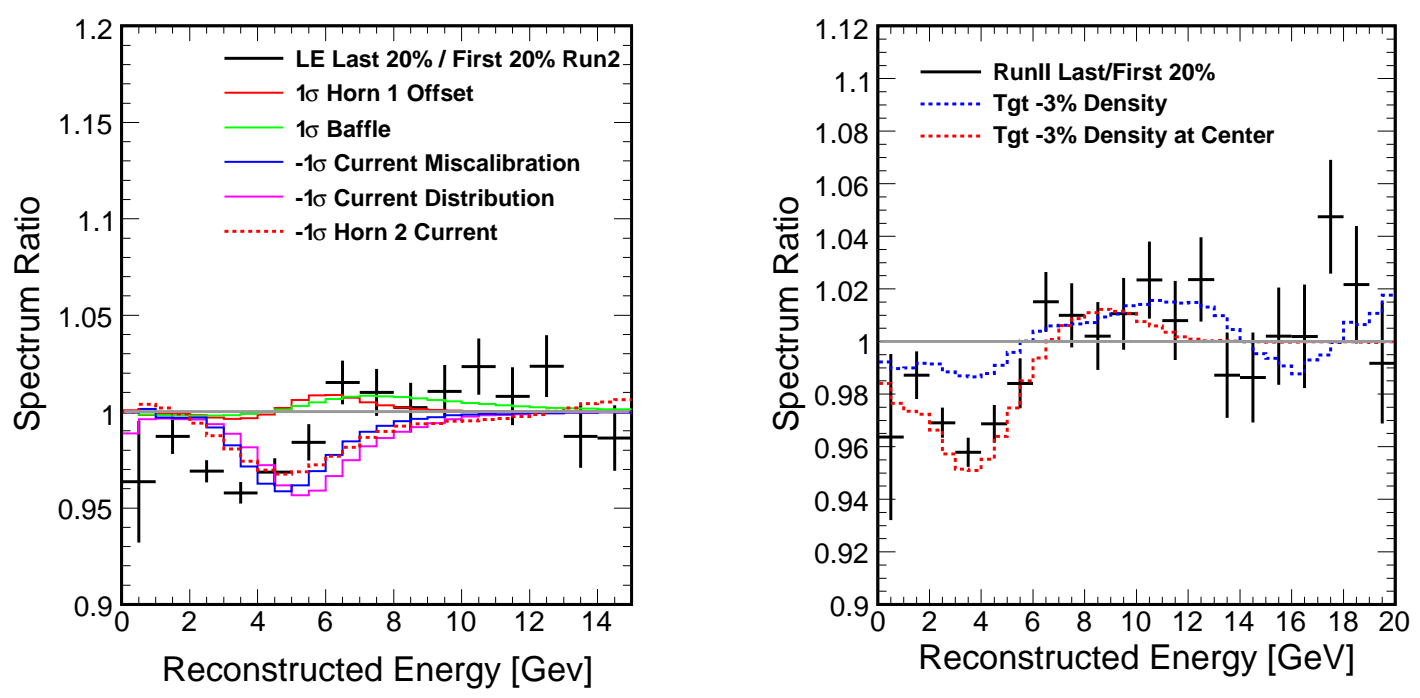

Figure 8.7: Left: The reduction of the flux from RunII is plotted with different focusing uncertainties. Right: The data is compared with two density loss models: an overall $3 \%$ density reduction (blue) and a $3 \%$ density reduction in the target center (red). The data is plotted as the ratio of the first $20 \%$ with the last $20 \%$.

Figure 8.7 shows the data for RunII with the $1 \sigma$ focusing uncertainties. To highlight the time dependent effect of the data, it is plotted as the ratio of the last $20 \%$ to the first $20 \%$ of RunII. The data plotted in this fashion shows a $4 \%$ decrease in the energy spectrum at $3 \mathrm{GeV}$ and a $1 \%$ increase from $6-10 \mathrm{GeV}$. All of these uncertainties affect the energy spectrum in the wrong place.

Another possible cause for this effect is that the target could suffer damage over time from large amounts of radiation resulting in a loss of target mass. Using the FLUKA Monte Carlo, the mass loss was modeled by reducing the density by $3 \%$ throughout the target and by reducing the density $3 \%$ in the center of the target. Figure 8.7 shows the results of these models and compares them to the nominal data. Both models show a reduction in the neutrino energy spectrum in the right place. They also predict a slight increase in the spectrum at higher energies.

A similar study [89] looked for evidence of a hole in the NuMI target. The study reweighted hadrons coming from different regions of the target to simulate a hole. Full 

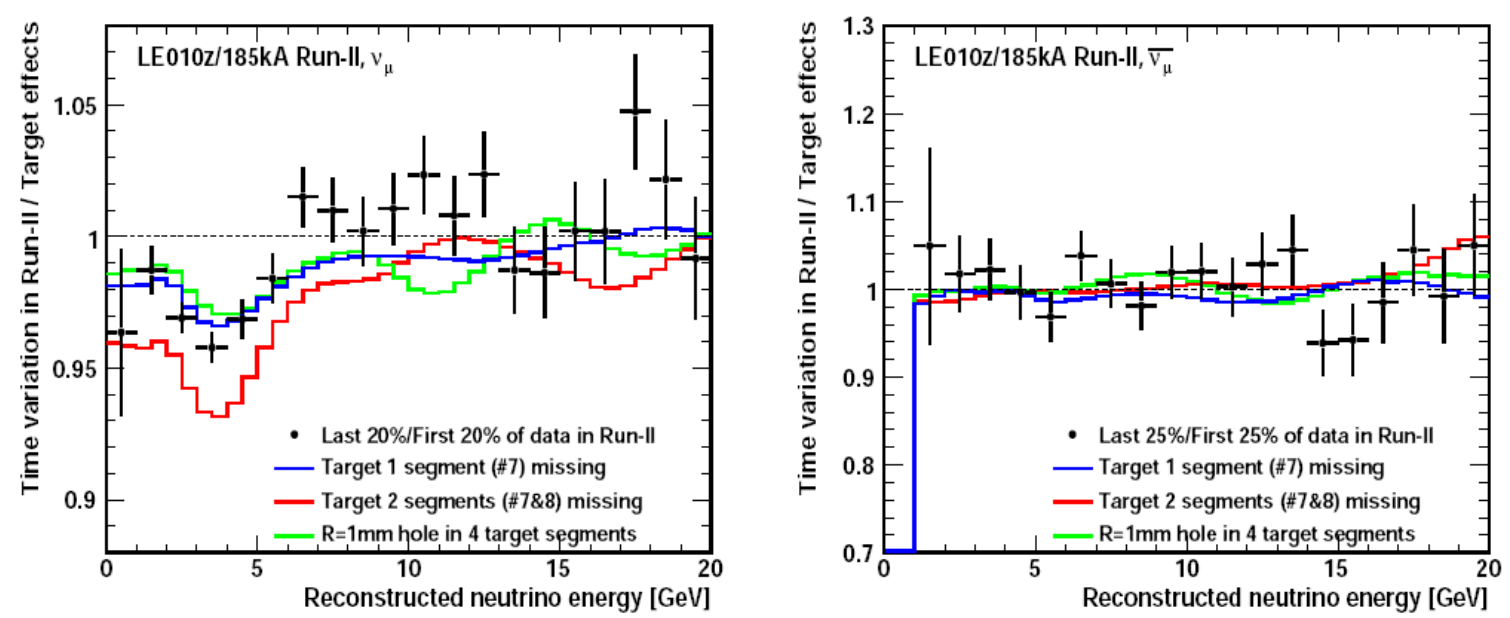

Figure 8.8: The RunII flux reduction is compared to different models simulating a hole in the target for $\nu_{\mu}$ (left) and $\bar{\nu}_{\mu}$ (right). Figure taken from [89].

FLUKA simulations were also done where different segments of the target were removed. These results, shown in Figure 8.8, were consistent with the density loss models - all of them showed the correct behavior for both $\nu_{\mu}$ and $\bar{\nu}_{\mu}$. These studies give strong evidence that the loss of flux could be due to radiation damage in the target.

External radiation tests done at Brookhaven National Laboratory [89] estimated that little damage occurred for graphite and carbon composites for radiation doses under $10^{21}$ protons $/ \mathrm{cm}^{2}$. However, just above that level, dramatic and serious degradations to the structure were seen. Unfortunately, the NuMI targets are not currently available for examination due to high radiation levels.

Figure 8.9 compares the difference seen in the RunII energy spectrum with the uncertainty in the Near Detector as calculated in Section 5.7. The change in the data is well within the calculated uncertainty so this systematic error was not considered in the oscillation analysis. This clearly needs to be understood for future analyses, especially if the trend continues. 


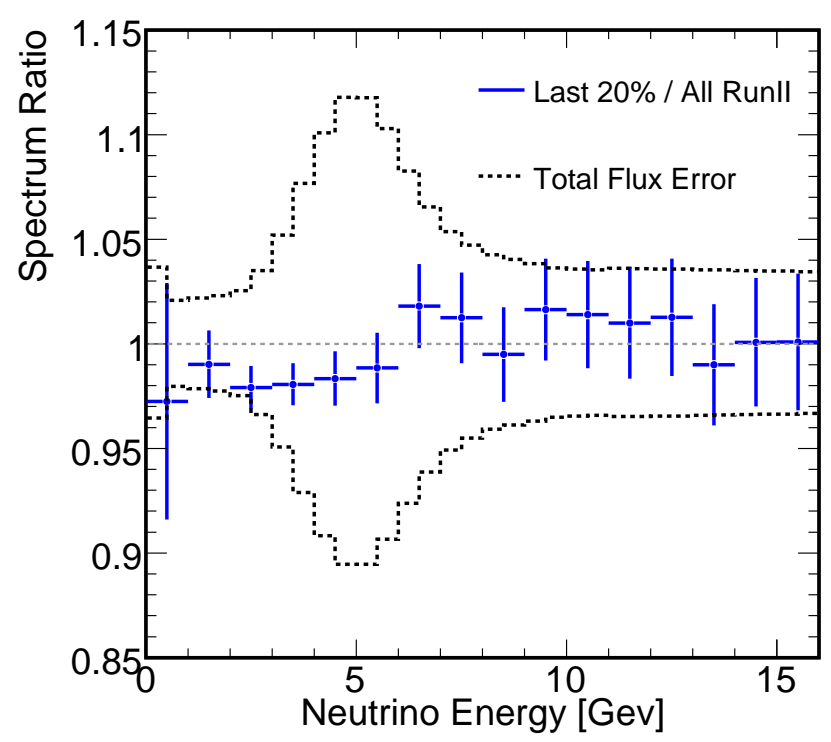

Figure 8.9: The dashed line shows the flux uncertainty for the Near Detector and the points show the ratio of the last $20 \%$ of RunII to all the data in RunII.

\subsection{Relative Normalization}

The uncertainty on the relative normalization between Near and Far has been estimated to be $4 \%$ [46]. This number has three sources: $2 \%$ from fiducial mass calculations, $1 \%$ from the live time, and $3 \%$ from reconstruction differences. To get a more realstic estimate on the reconstruction differences, nine scanners, including the author of this thesis, examined over 12,000 combined events in data and Monte Carlo for both detectors [90].

The normalization uncertainty from reconstruction can be calculated as:

$$
\text { Uncertainty }=1-\frac{\left(R_{\text {tracking }} \times R_{\text {sign }} \times R_{\text {fiducial }}\right)_{\text {Near }}}{\left(R_{\text {tracking }} \times R_{\text {sign }} \times R_{\text {fiducial }}\right)_{\text {Far }}}
$$

where $R_{i}$ is the ratio of the efficiencies between the data and Monte Carlo for track identification (tracking), charge sign determination (sign) and fiducial volume effects (fiducial).

The tracking efficiency estimates how often reconstructed tracks are misidentified in $\mathrm{CC}$ events and missed in $\mathrm{NC}$ events. The sign efficiency identifies tracks obviously 


\begin{tabular}{|c|c|c|c|c|c|c|}
\hline & ND MC & ND Data & FD MC & FD Data & $R^{N D}$ & $R^{F D}$ \\
\hline $\begin{array}{l}\text { NC-like } \\
\text { ETracking }\end{array}$ & $\begin{array}{c}0.9714 \pm \\
0.0030\end{array}$ & $\begin{array}{c}0.9726 \pm \\
0.0028\end{array}$ & $\begin{array}{c}0.9948 \pm \\
0.0013\end{array}$ & $\begin{array}{c}1^{+0}-0.0018 \text { or } \\
>0.9989 \\
(68 \% \text { CL })\end{array}$ & $\begin{array}{c}1.0013 \pm \\
0.0042\end{array}$ & $\begin{array}{c}1.0052 \pm \\
0.0022\end{array}$ \\
\hline $\begin{array}{l}\text { CC-like } \\
\text { ETracking }\end{array}$ & $\begin{array}{c}0.9826 \pm \\
0.0022\end{array}$ & $\begin{array}{c}0.9762 \pm \\
0.0023\end{array}$ & $\begin{array}{c}0.9876 \pm \\
0.0016\end{array}$ & $\begin{array}{c}0.9805 \pm \\
0.0061\end{array}$ & $\begin{array}{c}0.9934 \pm \\
0.0032\end{array}$ & $\begin{array}{c}0.9928 \pm \\
0.0064\end{array}$ \\
\hline $\begin{array}{c}\text { CC-like } \\
\varepsilon_{\mathrm{q}<0}\end{array}$ & $\begin{array}{c}0.9910 \pm \\
0.0022\end{array}$ & $\begin{array}{c}0.9974 \pm \\
0.0023\end{array}$ & $\begin{array}{c}0.9903 \pm \\
0.0016\end{array}$ & $\begin{array}{c}0.9973 \pm \\
0.0061\end{array}$ & $\begin{array}{c}1.0065 \pm \\
0.0032\end{array}$ & $\begin{array}{c}1.0071 \pm \\
0.0064\end{array}$ \\
\hline $\begin{array}{c}\text { CC-like } \\
\varepsilon_{\text {is_fid }}\end{array}$ & $\begin{array}{c}0.9929 \pm \\
0.0022\end{array}$ & $\begin{array}{c}0.9926 \pm \\
0.0023\end{array}$ & $\begin{array}{c}0.9987 \pm \\
0.0016\end{array}$ & $\begin{array}{c}0.9967 \pm \\
0.0061\end{array}$ & $\begin{array}{c}0.9997 \pm \\
0.0032\end{array}$ & $\begin{array}{c}0.9981 \pm \\
0.0064\end{array}$ \\
\hline
\end{tabular}

Figure 8.10: The efficiencies as calculated from the hand scan of events. The $R$-values from the last two columns give the ratio between the data and Monte Carlo. Taken from [90].

reconstructed with the wrong curvature. The fiducial efficiency accounts for events where the scanner found an incorrect vertex position changing whether or not the event was within the fiducial volume. Figure 8.10 lists the efficiencies and $R$ values.

The relative normalization uncertainty on the reconstruction was found to be $0.23 \%$. Assuming the $R$-values to be uncorrelated, the uncertainty is found by adding in quadrature the uncertainties on the $R$-values. Plugging in the uncertainties from Figure 8.10 gives an uncertainty of $1.3 \%$. Since the uncertainty is larger than the measurement, an upper limit is set of $1.3 \%$ on the normaliztion uncertainty from reconstruction. This study reduced the overall normalization uncertainty from $4 \%$ to $2.6 \%$.

\subsection{Hadronic Energy Scale}

The estimated error from the shower or hadronic energy scale consists of the absolute energy scale (including single hadron response, intranuclear effects, calibration) and the relative energy scale between Near and Far. Other differences such as steel and scintillator thicknesses are believed to be small compared to the calibration uncertainties. 


\begin{tabular}{lc}
\hline \hline Source & Uncertainty \\
\hline Tuning MC to CalDet data & $2.5-5 \%$ \\
CalDet beam & $2 \%$ \\
CalDet stopping muons & $1.4 \%$ \\
Spill vs. Cosmic Response & $1 \%$ \\
\hline Total & $5.6 \%$ \\
\hline \hline
\end{tabular}

Table 8.1: Uncertainties from the Calibration Detector for use in calculating the absolute hadronic energy scale.

\subsubsection{Absolute Energy Scale}

The Calibration Detector (see Section 3.2.3) provided the single hadron response for the MINOS detectors. Table 8.1 lists the different components of the uncertainty. A total uncertainty of $5.6 \%$ was derived for the absolute scale uncertainty.

\subsubsection{Final State Uncertainties}

Hadrons produced from neutrino interactions can re-interact in the target nucleus. This process is referred to as final state interactions or intranuclear re-scattering. The uncertainty in these final state interactions must be accounted for in the calculation of the resulting shower energy as measured in the MINOS detectors. The total uncertainty was estimated by selecting the important parameters and then shifting each component by $1 \sigma$ and evaluating the resulting shift in the shower energy. The total uncertainty from these effects is shown in Figure 8.11. The maximum contribution is $8.2 \%$ in the lowest energy bin and falls off at higher energies.

\subsubsection{Relative Uncertainty}

By comparing the calibration procedures in the Near and Far Detectors a relative uncertainty can be found. The total uncertainty in the Near Detector is $2.3 \%$ while the Far Detector is $2.4 \%$. Combining these in quadrature gives a Near-to-Far relative calibration of $3.3 \%$. The largest uncertainty in both the Near and Far detectors is the result of 


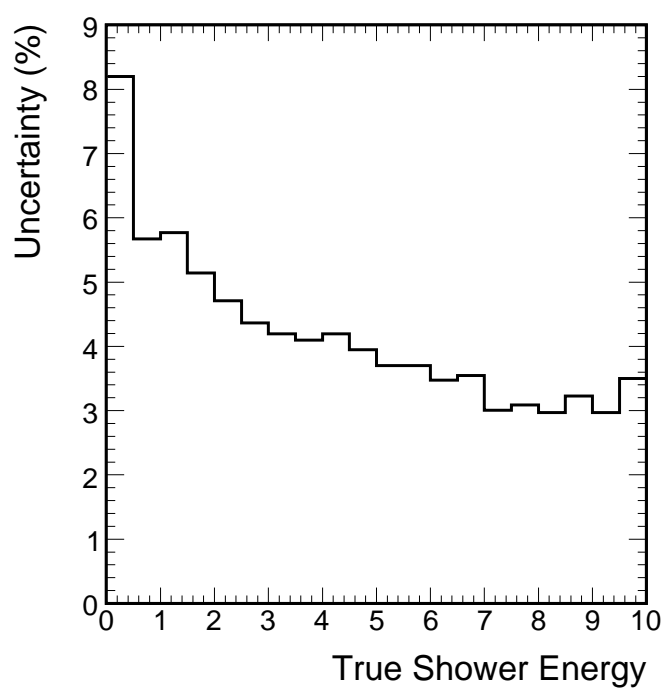

Figure 8.11: The uncertainty on the hadronic energy scale from final state interactions as a function of true shower energy.

differences in the spatial variation of the response when comparing the data to the Monte Carlo.

The total hadronic energy uncertainty is obtained by adding in quadrature the values from intranuclear re-scattering, 5.6\% uncertainty from CalDet, and the $2.4 \%$ uncertainty from the Far Detector calibration. This is a shower energy dependent value with a maximum of $10.3 \%$.

\subsection{Neutral Current Contamination}

The Monte Carlo cannot reliably model background NC events due to uncertainties in cross-sections and differences in the probability of finding a reconstructed track in data and Monte Carlo. This requires constraints from the Near Detector data to understand these uncertainties. John Marshall performed a data driven study to estimate the uncertainty on the NC Background [91] using muon removed events. Muon hits were removed from CC events to create NC-like or "fake" NC events. By comparing these fake NC events, an estimate of the track finding efficiency in hadronic showers can be obtained. 


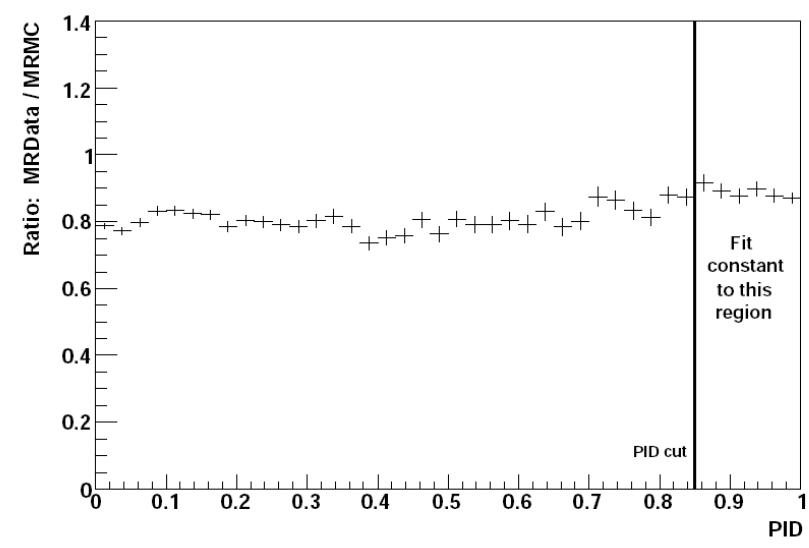

Figure 8.12: The ratio of selected muon removed data events to muon removed Monte Carlo events as a function of the PDF CC discriminator (labeled PID in the plot). The vertical line indicates the cut value for CC-like identification. Taken from [54]

The fake NC events are reprocessed through the reconstruction chain applying the CC event selection cuts (see Section 6.1.1), including having a reconstructed track. This sample can then be used to study differences in the reconstruction of background NC events. The Likelihood or PDF discriminator (see Section 7.3) is used to find CC-like events. The ratio of the PDF distribution for muon removed data and Monte Carlo above the CC-like cut at 0.85 is flat (see Figure 8.12).

Fitting the ratio above the cut value to a constant gives an efficiency correction factor. This factor is computed separately for different ranges of neutrino energy. The results of the fits gives an energy dependent correction factor that addresses the differences in finding tracks in NC events.

After the efficiency correction, a second "normalization" correction is carried out that scales the true NC events from the muon removed Monte Carlo sample to minimize differences between the data for the PDF distribution. The overall correction, combining the "normalization" and "efficiency" corrections, is $-7.9 \pm 3.5 \%$. The typical efficiency corrections are $\sim 20 \%$, while the typical normalization corrections $\sim 10 \%$. Assuming these numbers are uncorrelated, the NC background is calculating to be $25 \%$. Given that 
muon removed events will have inherent differences from true neutral current events, the value was doubled to $50 \%$.

The fit in the Near Detector data (see Chapter 5.3.3) also included a scale factor for the NC component. A separate factor for $\nu_{\mu}$ and $\bar{\nu}_{\mu}$ was included in the fit with $30 \%$ errors in the penalty terms of the $\chi^{2}$. The scale factors from the fit were $14.0 \pm 9.1 \%$ for $\nu_{\mu}$ and $-36.8 \pm 25.3 \%$ for $\bar{\nu}_{\mu}$. These numbers are the amount the Monte Carlo must be changed to agree with the Near Detector Data. These numbers fall within the $50 \%$ uncertainty calculated above.

\subsection{Muon Momentum}

A Range-Curvature Task Force studied the systematic error on the muon energy as measured by range [92]. They found the error to be $2 \%$ by comparing GEANT Monte Carlo predicitons to external data. To establish the uncertainty on the momentum determination from curvature a comparison of the muon energy from range and curvature was carried out. The agreement was found to be good with 1\%, making the total systematic error on the muon energy from curvature to be $3 \%$.

\subsection{Cross-Section}

Estimates for the uncertainty of the charged current neutrino cross-section can be broken down into different parts: uncertainties on the quasi-elastic and resonance axial mass $M_{A}$ and uncertainties on the behavior of the cross-section in the transition region from resonance to deep inelastic scattering.

The low energy region is governed by the two $M_{A}$ values. Both are dipole parametrization of the neutrino-nucleon cross-section. The value of $M_{A}^{Q E}$ and $M_{A}^{R E S}$ used in the simulation, based on preexisting data, are 0.99 and 1.12 respectively. Both of these measurements have an uncertainty of $15 \%$ which takes into account evidence that the data 


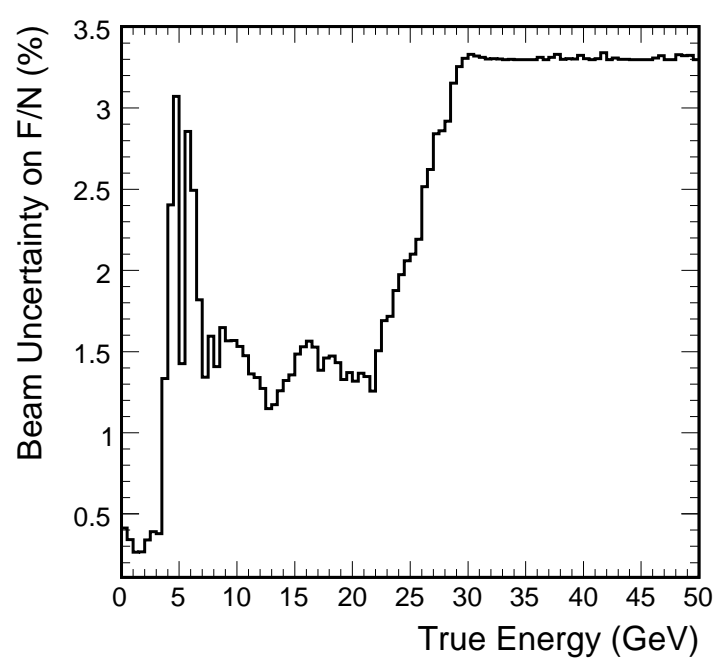

Figure 8.13: The uncertainty on the Far/Near ratio from the flux calculation of Chapter 5 for the LE configuration.

may not be described by the simple dipole parametrization and effects in iron nuclei. These classes of events make up a majority of the events in the oscillation region, and therefore uncertainties for DIS events are not considered.

\subsection{Flux}

The uncertainty in the flux was calculated in Section 5.7.2 from the Near Detector fit. For completeness, Figure 8.13 shows the total uncertainty on the Far/Near ratio for the LE beam configuration.

\subsection{Extrapolation Errors}

Each extrapolation technique will have different errors associated with it. To estimate the statistical errors incurred from the Near Detector fit, a procedure similar to that used in Section 5.7.1 is used. The differences will be that all of the Near Detector fit parameters will be included and the prediction will be on the reconstructed energy spectrum. The procedure samples a large number of random parameter values chosen based on the error 
matrix of the Near Detector fit. For each set of parameter values, a prediction of the Far Detector $\nu_{\mu} \mathrm{CC}$ energy spectrum is made by reweighting the same Monte Carlo sample. From this set of predictions a bin-to-bin covariance matrix is made.

Figure 8.14 shows the resultant correlation matrices for the case when only the hadron production parameters are varied, the detector parameters and both. The errors for each bin of reconstructed energy are taken as the square root of the diagonal elements of the covariance matrix and shown in Figure 8.15 for the LE configuration. The lowest energy bin exhibits large errors compared to the other bins because of the large NC background in that bin.

\subsection{Residual Difference from Near Detector Fit}

The extrapolation method that uses the fit in the Near Detector depends on how well the tuned Monte Carlo matches the data. Any residual differences after the fit will be propagated to the unoscillated prediction in the Far Detector. Figure 8.16 shows the ratio of the data to Monte Carlo for the LE RunI data. It shows a difference of $\sim 5 \%$ from $10-15 \mathrm{GeV}$ and $\sim 3 \%$ from $17-30 \mathrm{GeV}$.

\subsection{Impact on the Oscillation Measurements}

The systematic errors discussed above can be separated into those that are global and affect both the Near and Far Detectors and those that are only relative. The Near Detector provides constraints on the global systematic errors by using the Near Detector fit.

\subsubsection{Global Errors}

The strategy used to quantify the impact of the systematic errors on the oscillation parameters proceeds as follows. 

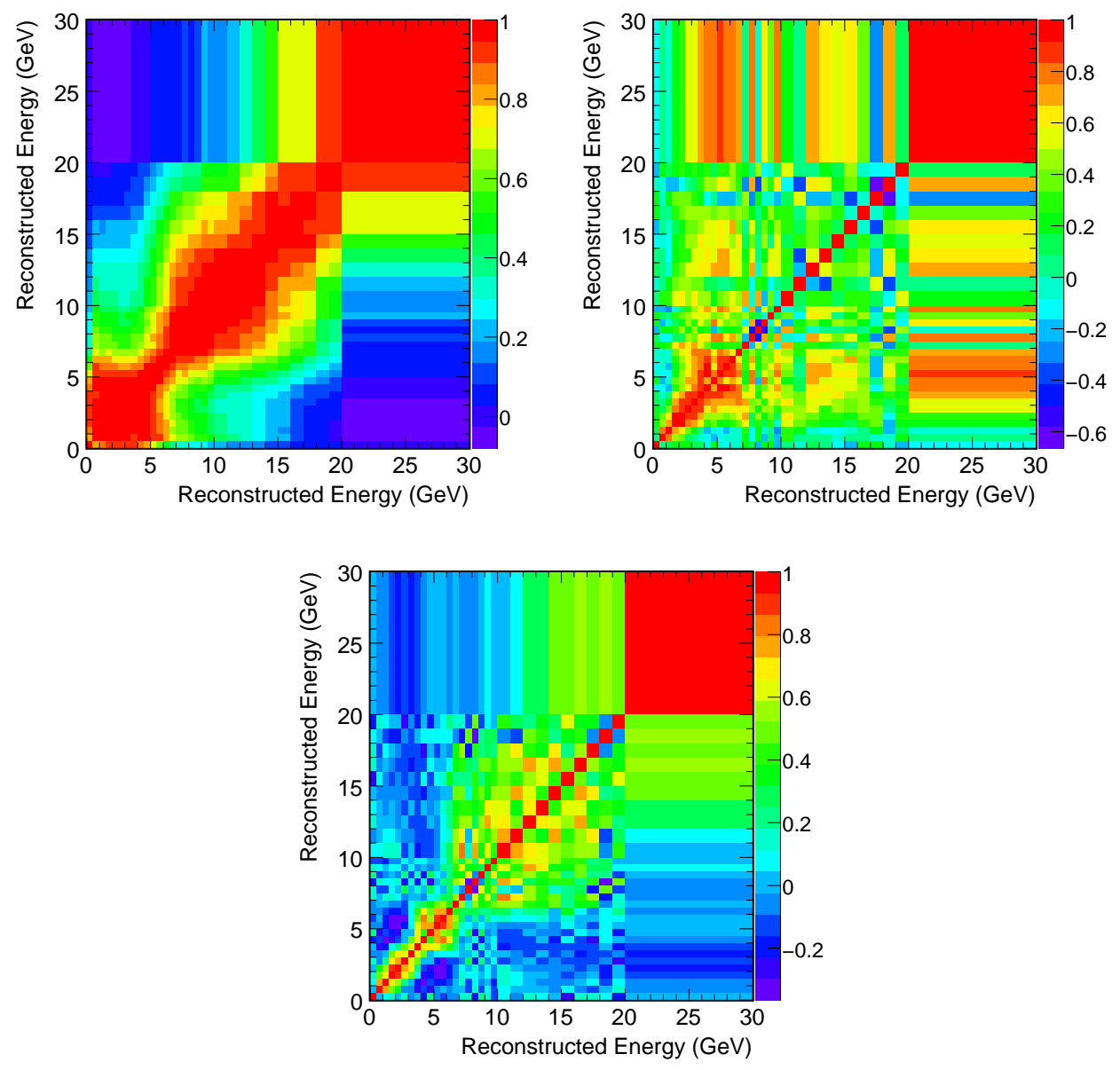

Figure 8.14: The correlation matrices between different bins in reconstructed energy for the Far Detector as predicted from the Near Detector fit. Top Left: Only the hadron production parameters are varied. Top Right: Only the detector parameters are varied. Bottom: All of the parameters are varied. together. 


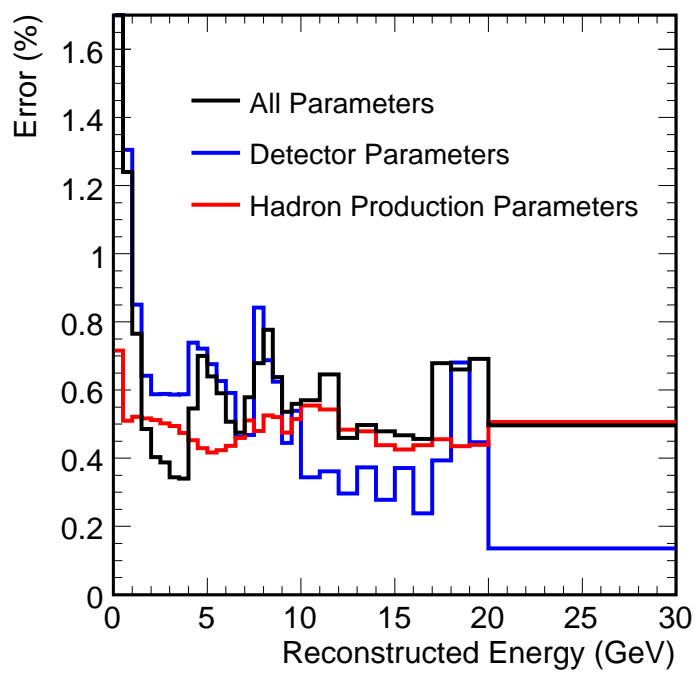

Figure 8.15: The percentage error on the LE energy spectrum as a function of reconstructed energy from the extrapolation. The black line is when all parameters are varied, the blue for detector parameters and the red for hadron production parameters.

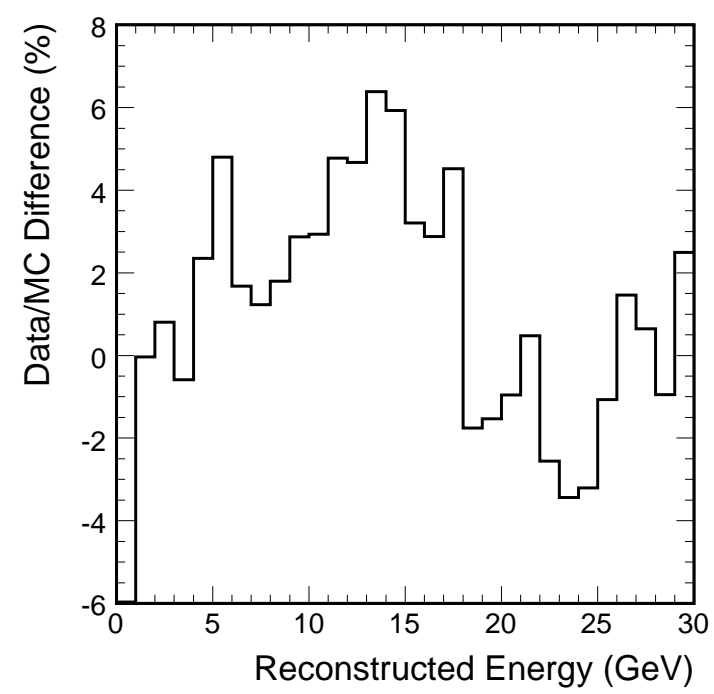

Figure 8.16: The difference between the data and Monte Carlo after the Near Detector fit as a function of reconstructed energy. 
1. In the Near Detector create a "fake" data set from the Monte Carlo.

2. Apply $1 \sigma$ systematic shifts of the important uncertainties to the Near Detector fake data.

3. Do a completely new fit to the Near Detector fake data as described in Section 5 to obtain an extrapolation to the Far Detector.

4. Create a fake data set in the Far Detector. Oscillate the data using the input parameters of $\sin ^{2}\left(2 \theta_{32}\right)=1.0$ and $\Delta m_{32}^{2}=2.44 \times 10^{-3} \mathrm{eV}^{2}$. Apply the same systematic shift as was done in the Near Detector fake data.

5. Use the extrapolation derived from the new Near Detector fit to predict the unoscillated Far Detector spectrum.

6. Fit the Monte Carlo to the fake Far Detector data and find out how far the oscillation parameters have shifted from the input values.

The first three steps attempt to see how well the Near Detector fit can recover and reproduce shifts that was done in the fake data. Because of correlations between variables, it will not necessarily be able to find the correct value. The global errors evaluated in this study are: the absolute hadronic energy scale, the muon energy scale, the neutral current background, and the $M_{A}$ values for QE and RES. For the hadronic energy scale two different values are used. The first, is a flat $10.3 \%$ correction and the second, the parametrization of the hadronic energy uncertainty found in Section 5.3.3.

Table 8.2 shows how well the fit was able to recover the shift applied to the fake data. In all cases the fit was able to fit for the correct shift. To test the robustness of this procedure, a few of the parameters were run with the exact same procedure except that the fake data was first reweighted with the values from the Near Detector fit of Chapter 5. Table 8.3 lists how well the fit can describe these shifts. In general, it is not able to recover the correct systematic errors as well as the bare Monte Carlo method 


\begin{tabular}{lc}
\hline \hline Parameter Shift & Best Fit Value \\
\hline Neutral Current $+50 \%$ & $+48 \%$ \\
Neutral Current $-50 \%$ & $-48 \%$ \\
Shower Parametrization $+1 \sigma$ & $+1.0 \sigma$ \\
Shower Parametrization $-1 \sigma$ & $-1.0 \sigma$ \\
Shower Scale $+10.3 \%$ & $1.0 \sigma$ \\
Shower Scale $-10.3 \%$ & $+10.3 \%$ \\
Muon Scale $+2 \%$ & $+2 \%$ \\
Muon Scale $-2 \%$ & $-2 \%$ \\
Shower Parametrization $-1 \sigma$ & $-1.0 \sigma$ \\
$M_{A}$ QE $+15 \%$ & $14.4 \%$ \\
$M_{A}$ QE $-15 \%$ & $-14.5 \%$ \\
$M_{A}$ RES $+15 \%$ & $14.7 \%$ \\
$M_{A}$ RES $-15 \%$ & $-14.8 \%$ \\
\hline \hline
\end{tabular}

Table 8.2: The values obtained by shifting a set of fake Near Detector data and doing a completely new Near Detector fit. The left column lists the systematic shift that was applied to the fake data and the right column lists how well the fit was able to measure the corresponding shift.

\begin{tabular}{lc}
\hline \hline Parameter Shift & Best Fit Value \\
\hline Neutral Current $+50 \%$ & $41 \%$ \\
Neutral Current -50\% & $-68 \%$ \\
Shower Energy $1 \sigma$ & $1.3 \sigma$ \\
Shower Energy -1 $\sigma$ & $-0.64 \sigma$ \\
$M_{A} \mathrm{QE}+15 \%$ & $16.3 \%$ \\
$M_{A} \mathrm{QE}-15 \%$ & $-13.4 \%$ \\
\hline \hline
\end{tabular}

Table 8.3: The values obtained by shifting a set of fake Near Detector data and doing a completely new Near Detector fit. The left column lists the systematic shift that was applied to the fake data and the right column lists how well the fit was able to measure the corresponding shift. Here, the fake data was first reweighted by applying the results of the Near Detector fit described in Chapter 5. 


\begin{tabular}{lcc}
\hline \hline Parameter Shift & $\begin{array}{c}\text { Shift in } \Delta m_{32}^{2} \\
\left(10^{-5} \mathrm{eV}^{2}\right)\end{array}$ & $\begin{array}{c}\text { Shift in } \sin ^{2}\left(2 \theta_{32}\right) \\
\left(10^{-3}\right)\end{array}$ \\
\hline Neutral Current $+50 \%$ & -0.02 & 0.9 \\
Neutral Current $-50 \%$ & 0.2 & -0.06 \\
Shower Parametrization $+1 \sigma$ & -0.006 & 0.9 \\
Shower Parametrization $-1 \sigma$ & -0.007 & -0.03 \\
Shower Energy +10.3\% & -0.05 & -0.3 \\
Shower Energy -10.3\% & -0.06 & -0.4 \\
Track Energy $+2 \%$ & 0.01 & 0.02 \\
Track Energy -2\% & -0.01 & -0.003 \\
$M_{A}$ QE $+15 \%$ & 0.1 & 0.6 \\
$M_{A}$ QE - $15 \%$ & 0.09 & 0.2 \\
$M_{A}$ RES $+15 \%$ & 0.02 & -0.4 \\
$M_{A}$ RES - $15 \%$ & 0.02 & 0.06 \\
\hline \hline
\end{tabular}

Table 8.4: The shift in oscillation parameters produced by the corresponding shift in systematic errors.

Each fit in the Near Detector to the different systematic errors construct gives a different unoscillated prediction of at the Far Detector. This unoscillated prediction is used along with the fake Far Detector data set to see how much the oscillation parameters are shifted. Table 8.4 lists the shifts on $\Delta m_{32}^{2}$ and $\sin ^{2}\left(2 \theta_{32}\right)$ when using the nominal Monte Carlo and a graphical representation of these shifts is illustrated in Figure 8.17. The corresponding shifts on the oscillation parameters for the case when the fake data is reweighted by the Near Detector fit before a new fit is done are given in Table 8.5 and Figure 8.18. 


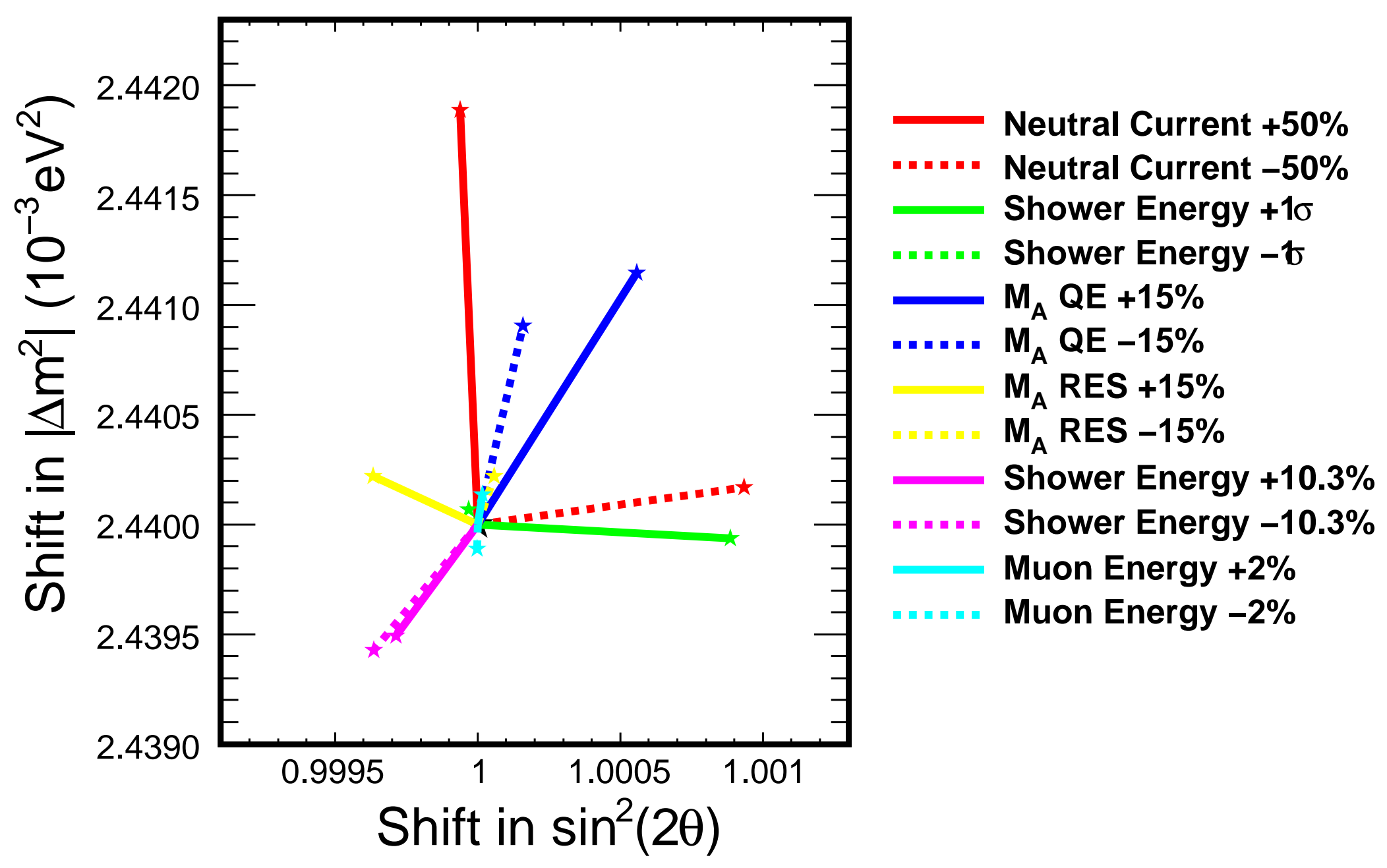

Figure 8.17: The shift in oscillation parameter space from the best fit point for different systematic errors. 


\begin{tabular}{lcc}
\hline \hline Parameter Shift & $\begin{array}{c}\text { Shift in } \Delta m_{32}^{2} \\
\left(10^{-5} \mathrm{eV}^{2}\right)\end{array}$ & $\begin{array}{c}\text { Shift in } \sin ^{2}\left(2 \theta_{32}\right) \\
\left(10^{-3}\right)\end{array}$ \\
\hline Neutral Current $+50 \%$ & 0.3 & -0.7 \\
Neutral Current $-50 \%$ & 0.05 & 1.2 \\
Shower Parametrization $1 \sigma$ & 0.3 & 0.1 \\
Shower Parametrization $-1 \sigma$ & 0.2 & 0.4 \\
$M_{A}$ QE $+15 \%$ & 0.06 & 1.0 \\
$M_{A}$ QE -15\% & 0.01 & 0.3 \\
\hline \hline
\end{tabular}

Table 8.5: The shift in oscillation parameter space from the best fit point for different systematic errors. Here, the fake data in the Near Detector was first reweighted by applying the results of the Near Detector fit described in Chapter 5. 


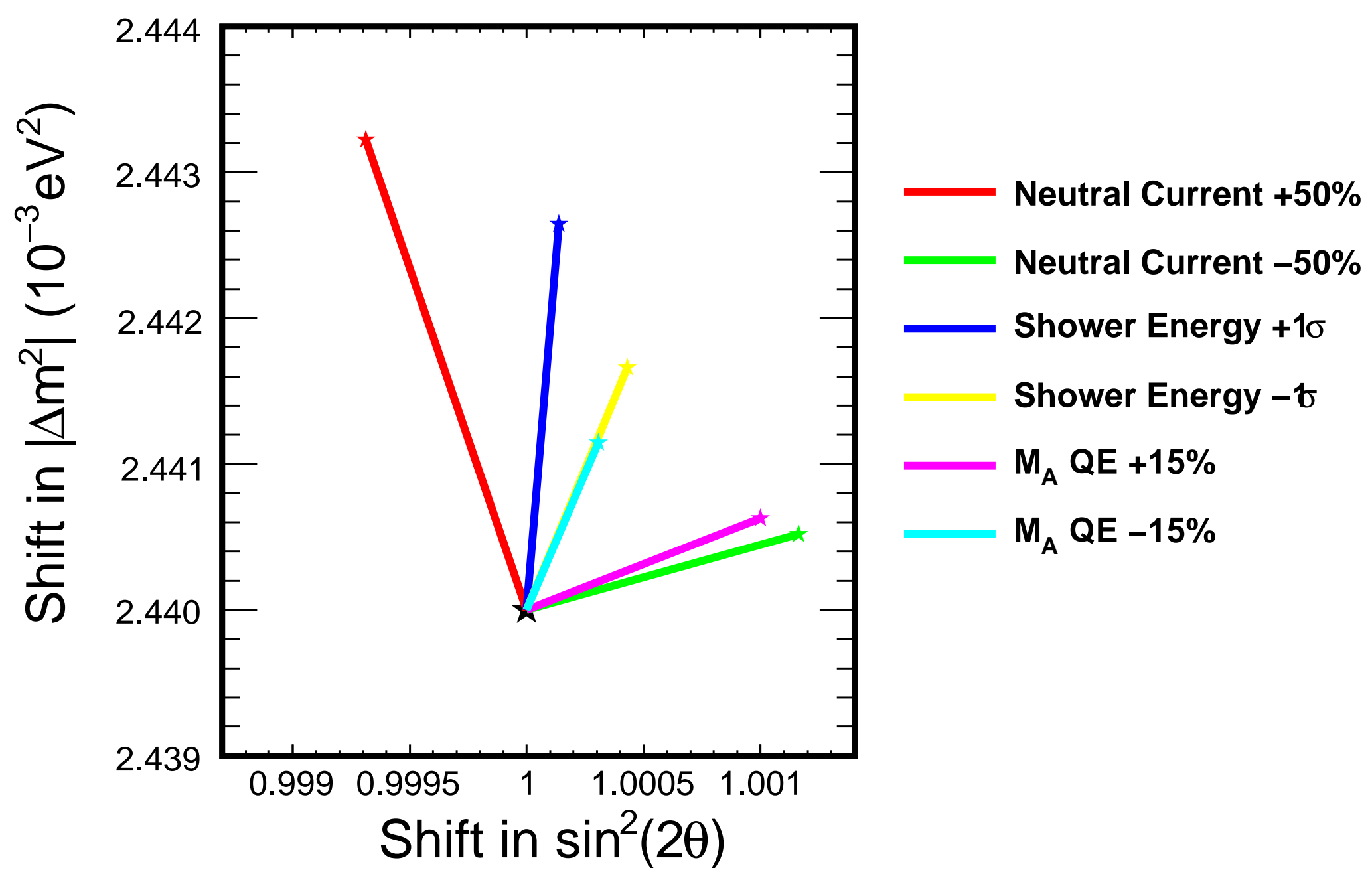

Figure 8.18: The shift in oscillation parameter space from the best fit point as a result of different systematic errors. Here, the fake data in the Near Detector was first reweighted by applying the results of the Near Detector fit described in Chapter 5. 


\begin{tabular}{lcc}
\hline \hline Parameter Shift & $\begin{array}{c}\text { Shift in } \Delta m_{32}^{2} \\
\left(10^{-5} \mathrm{eV}^{2}\right)\end{array}$ & $\begin{array}{c}\text { Shift in } \sin ^{2}\left(2 \theta_{32}\right) \\
\left(10^{-3}\right)\end{array}$ \\
\hline Normalization $+4 \%$ & -7.2 & -1.4 \\
Normalization $+4 \%$ & 7.3 & 1.5 \\
Normalization $+2.6 \%$ & -4.7 & -0.9 \\
Normalization $+2.6 \%$ & 4.7 & 1.0 \\
Extrapolation $+1 \sigma$ & -0.7 & -0.8 \\
Extrapolation $+1 \sigma$ & 0.7 & 0.8 \\
F/N Beam Error $+1 \sigma$ & -2.6 & 2.9 \\
F/N Beam Error $+1 \sigma$ & 2.6 & -2.9 \\
Data/MC difference in ND $+1 \sigma$ & -3.7 & 8.7 \\
Data/MC difference in ND $+1 \sigma$ & 3.7 & -8.1 \\
Relative Shower Energy $3.3 \%$ & 2.2 & 6.5 \\
Relative Shower Energy $3.3 \%$ & -2.2 & -6.2 \\
\hline \hline
\end{tabular}

Table 8.6: The shift in oscillation parameters produced by the corresponding shift in systematic errors for the relative systematic errors.

\subsubsection{Relative Errors}

Relative errors are simpler and do not need to go through the complication of the fit in the Near Detector. The relevant errors that have already been discussed are uncertainties due to: the relative shower energy differences, the extrapolation procedure, the beam or flux uncertainty, the normalization uncertainty and the residual differences in the data/Monte Carlo ratio from the Near Detector fit. For the normalization uncertainties two values will be used: $2.6 \%$ and $4 \%$. The $2.6 \%$ is the improved estimation of the uncertainty after the hand scan was done while $4 \%$ was the previous estimate. An energy dependent normalization uncertainty will also be considered where the the full reconstruction efficiency uncertainty of $1.3 \%$ is used as a linear function of energy. 


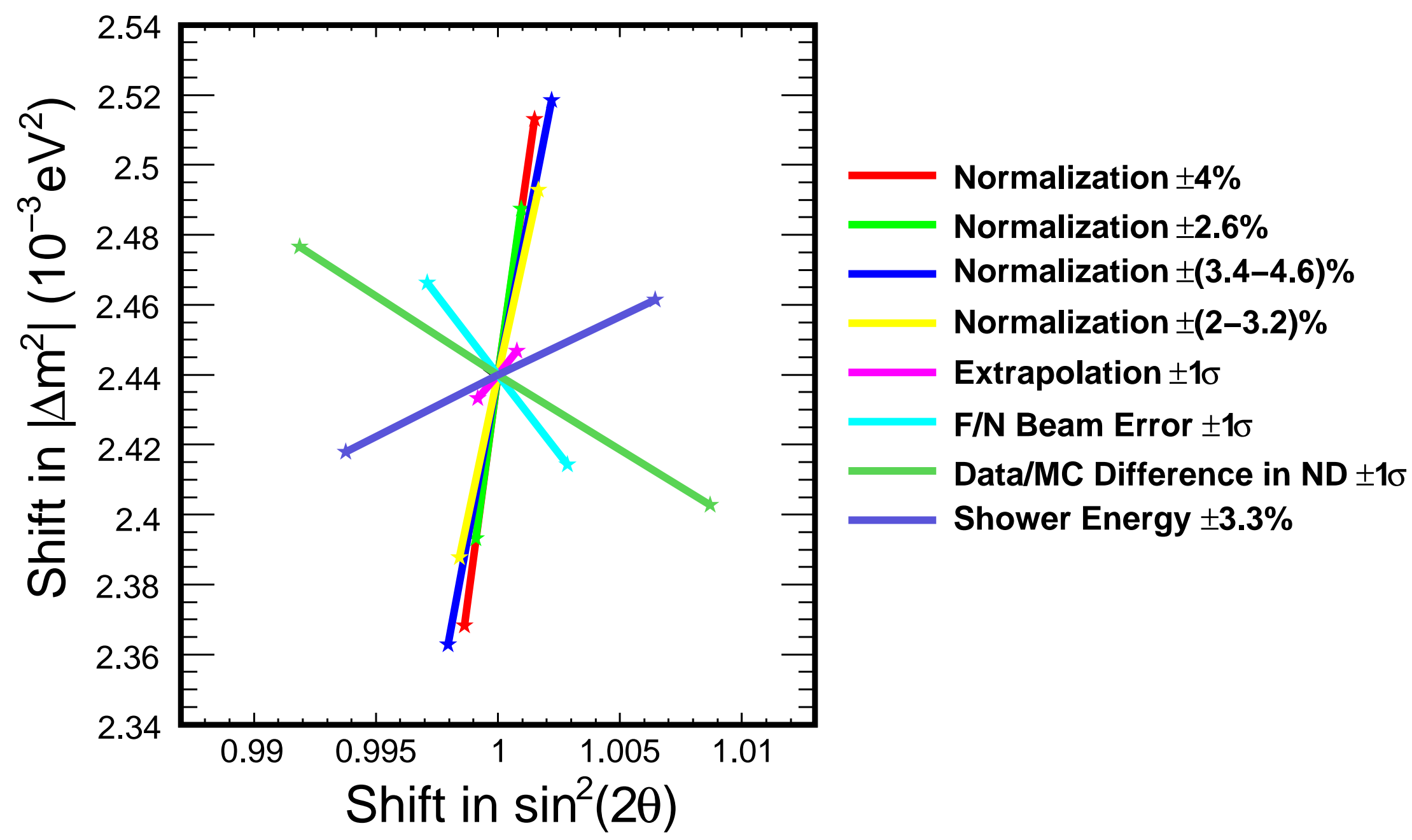

Figure 8.19: The shift in oscillation parameter space from the best fit point for the relative systematic errors 


\subsection{Discussion}

Comparing the size of the relative and global errors shows that the global errors are an order of magnitude smaller. This is due to the constraints provided by doing the fit to the data in Near Detector. In one sense the global fits may be contrived in that the Monte Carlo is only tweaked by a few parameters at a time and it is good at identifying these changes in the Near Detector.

While the global systematic errors are much smaller, they may not be able to be entirely trusted at the level indicated. They presuppose a good knowledge of each uncertainty and that all the major uncertainties are included in the fit. If this is not the case then they will be larger than these studies have shown. That said, the global errors should still be smaller than the relative errors. The largest relative errors are the residual Near Detector data/Monte differences, the relative normalization and the relative shower energy. These three systematic parameters will be included as nuisance parameters in the next section when the Far Detector data is analyzed. 


\section{Chapter 9}

\section{Analysis of the Far Detector Data}

With the extrapolation procedure defined and the systematic errors evaluated, the Far Detector data can now be analyzed. This chapter describes the selection of events in the Far Detector fits the data to the oscillation hypothesis.

The data sets used for the oscillation analysis were the 3 beam configurations LE RunI, RunII and HE RunII. The total number of protons on target (POT) from these configurations is $3.36 \times 10^{20}$. Table 9.1 lists the relative contribution from each configuration.

\begin{tabular}{lc}
\hline \hline Beam Configuration & POT $\left(\times 10^{20}\right)$ \\
\hline le010z185i RunI & 1.27 \\
le010z185i RunII & 1.94 \\
le250z200i RunII & 0.15 \\
\hline Total & 3.36 \\
\hline \hline
\end{tabular}

Table 9.1: Amount of data from each beam configuration used for the oscillation analysis. 


\subsection{Selection Cuts}

All physics runs in the Far Detector longer than 2 minutes and with greater than 100 triggers were selected. The following pre-selection cuts were applied to select neutrino events from the NuMI beam:

- Data Quality - Require the high voltage, coil and timing system to be fully operational.

- Spill Timing Cut - Require the time difference between an event and the nearest spill to be $\leq 20 \mu$ s and $\geq-30 \mu s$. Figure 9.1 shows this time difference for neutrino event candidates.

- Light Injection Cut - The calibration system uses an LED light injection system to characterize the detector response. An algorithm identifies light injection events and removes them from the data sample.

- Require a Track - Events must have at least one track that passes the track fitter.

- Fiducial Volume - The fiducial volume of the Far Detector is defined such that the $z$ position of the vertex must be between planes 3 and 239 or 252 and 464 . The distance, $d$, from the vertex to the center of the detector must satisfy $d>0.4 \mathrm{~m}$ to eliminate events close to the magnetic coil and $d<\sqrt{14}$.

- Track Direction - Since neutrino events should point back to Fermilab, the angle between the reconstructed track and the beam direction is required to be within $53^{\circ}$

- Charged Current Selection - Select CC events by requiring the output of the kNN algorithm, (see Section 7), be greater than 0.3.

Table 9.2 shows the fraction of events remaining after each of these cuts. The total number of events after the selection cuts is 848, 729 from the LE configurations and 118 from HE. The total event rate as a function of time is flat as shown in Figure 9.2. 

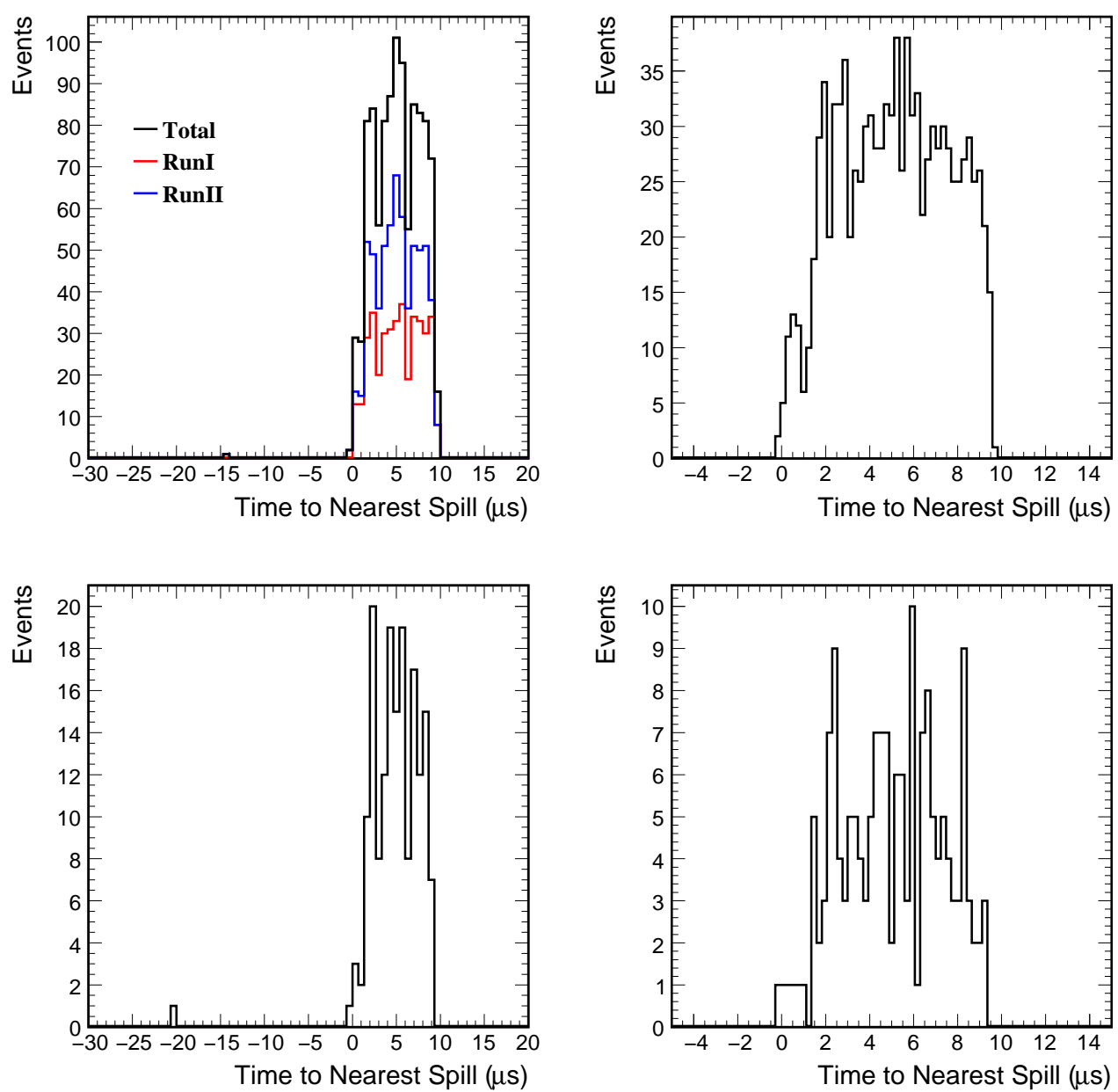

Figure 9.1: The relative time between neutrino events and the closest spill time. The top left shows the LE beam data for the total (black), RunI (red), and RunII (blue). The top right is a zoomed in view of the same plot. The bottom two plots show the same for the HE beam configuration. 

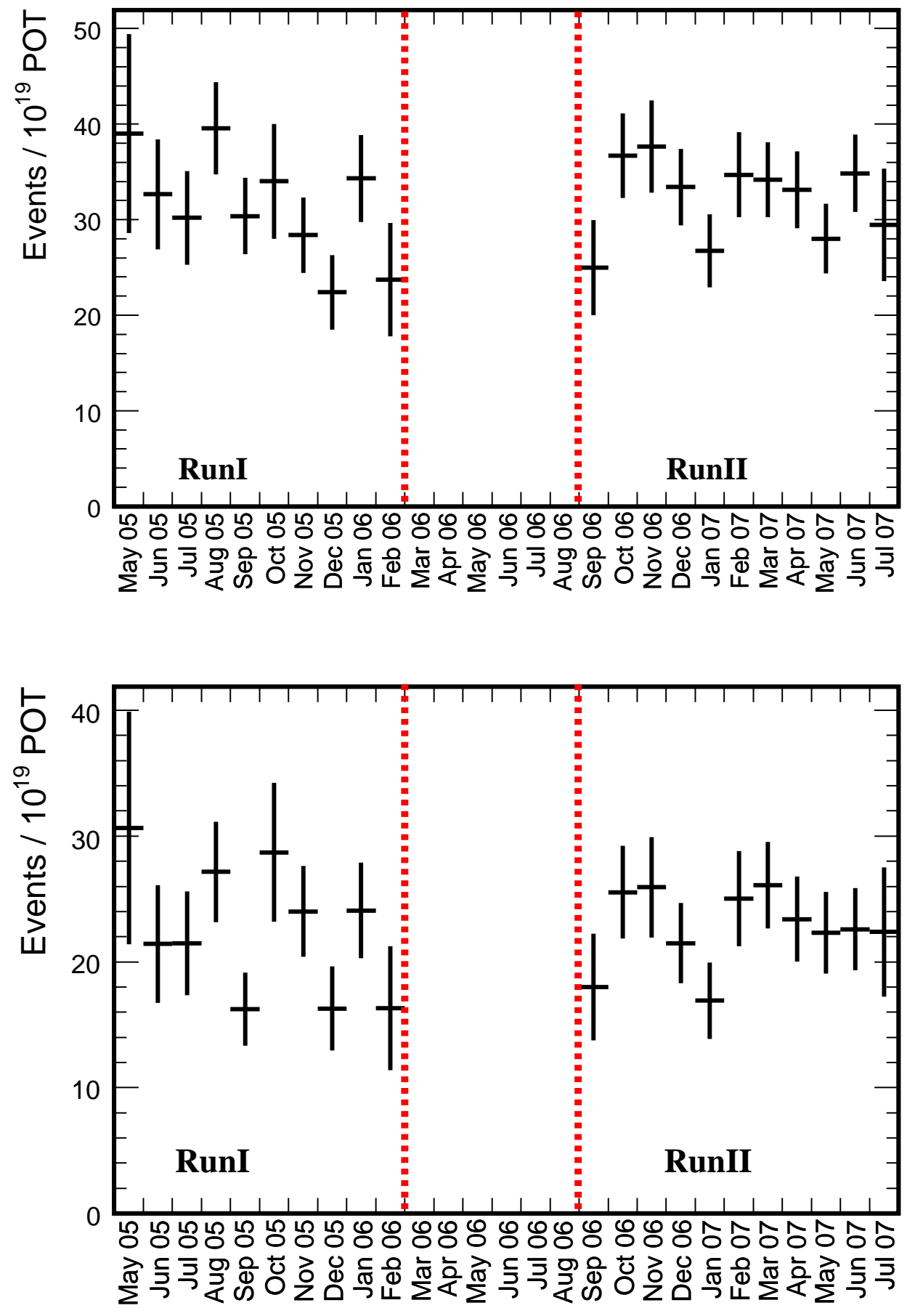

Figure 9.2: The number of total neutrino events as a function of month is shown at the top, and the number of CC-like events on the bottom. 


\begin{tabular}{lcccc}
\hline \hline Cut & LE Runs & Remaining (\%) & HE Run & Remaining (\%) \\
\hline Track in Fiducial & 1157 & 100 & 183 & 100 \\
Data Quality & 1141 & 99 & 175 & 96 \\
Timing Cut & 1113 & 96 & 174 & 95 \\
Direction Cut & 1063 & 92 & 170 & 93 \\
Beam Quality & 1037 & 90 & 168 & 92 \\
Track Quality & 1034 & 89 & 167 & 91 \\
Track Charge & 863 & 75 & 139 & 76 \\
Charged Current & 730 & 63 & 118 & 64 \\
Energy $\leq$ 200GeV & 729 & 63 & 118 & 64 \\
\hline \hline
\end{tabular}

Table 9.2: The effect of different cuts on the number of events in the Far Detector for the LE and HE beam configurations.

\section{$9.2 \quad$ Blinding}

MINOS conforms to a blind analysis policy where a fraction of the data is hidden until all analysis algorithms have been defined. This reduces biases in the analysis as a result of previous measurements. All of the Near Detector is open for al to analyze as well as non-spill data from the Far Detector. Far Detector data taken during beam spills is split into two separate streams by a selection algorithm. One stream is open and the other is "blinded" or hidden. The only valid reason to look at the blind set is to check for possible calibration or reconstruction problems. The blinding algorithm selects events using a sinusoidal function of the total pulse height and event length where the phase and frequency is chosen at random. The plots in the rest of this section use the full Far Detector data, but were not made until after the the analysis had been finalized and approved.

\subsection{Data in the Far Detector}

Figures 9.3 to 9.10 compare various distributions of the Far Detector data and the Monte Carlo for the LE and HE configurations. These comparisons include an unoscillated 
prediction using the extrapolation procedure defined in 6.2 and a fit to the oscillation hypothesis. Clear evidence is seen of a suppression of the low energy events. 

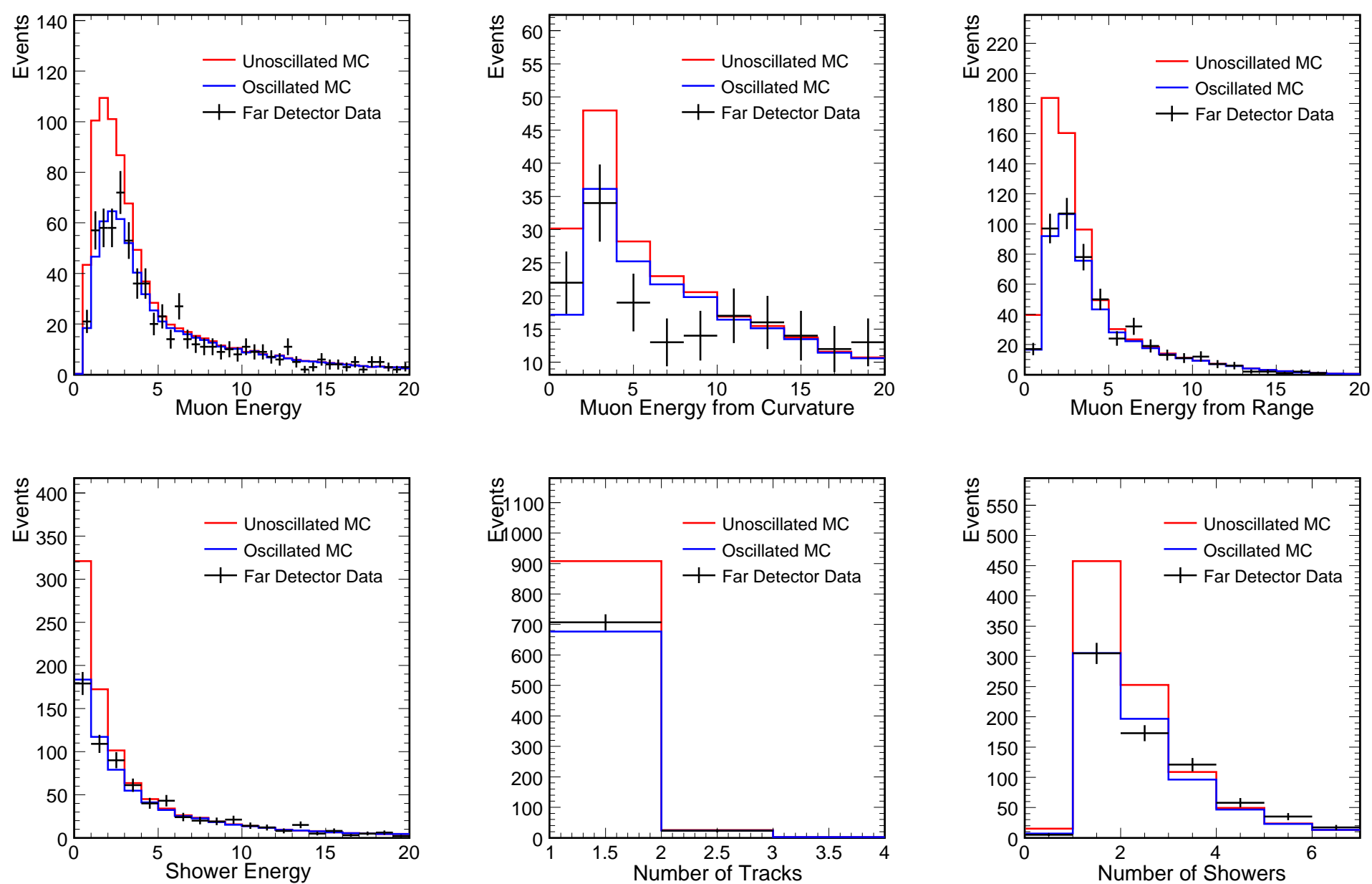

Figure 9.3: Far Detector plots of muon and shower quantities for the LE beam. The black points are the data, the red line the unoscillated prediction and the blue the best fit oscillation result. Shown are the muon energy (top left), the muon energy from exiting tracks (top middle), muon energy from contained tracks (top right), shower energy (bottom left), the number of tracks (bottom middle), the number of showers (bottom right). 

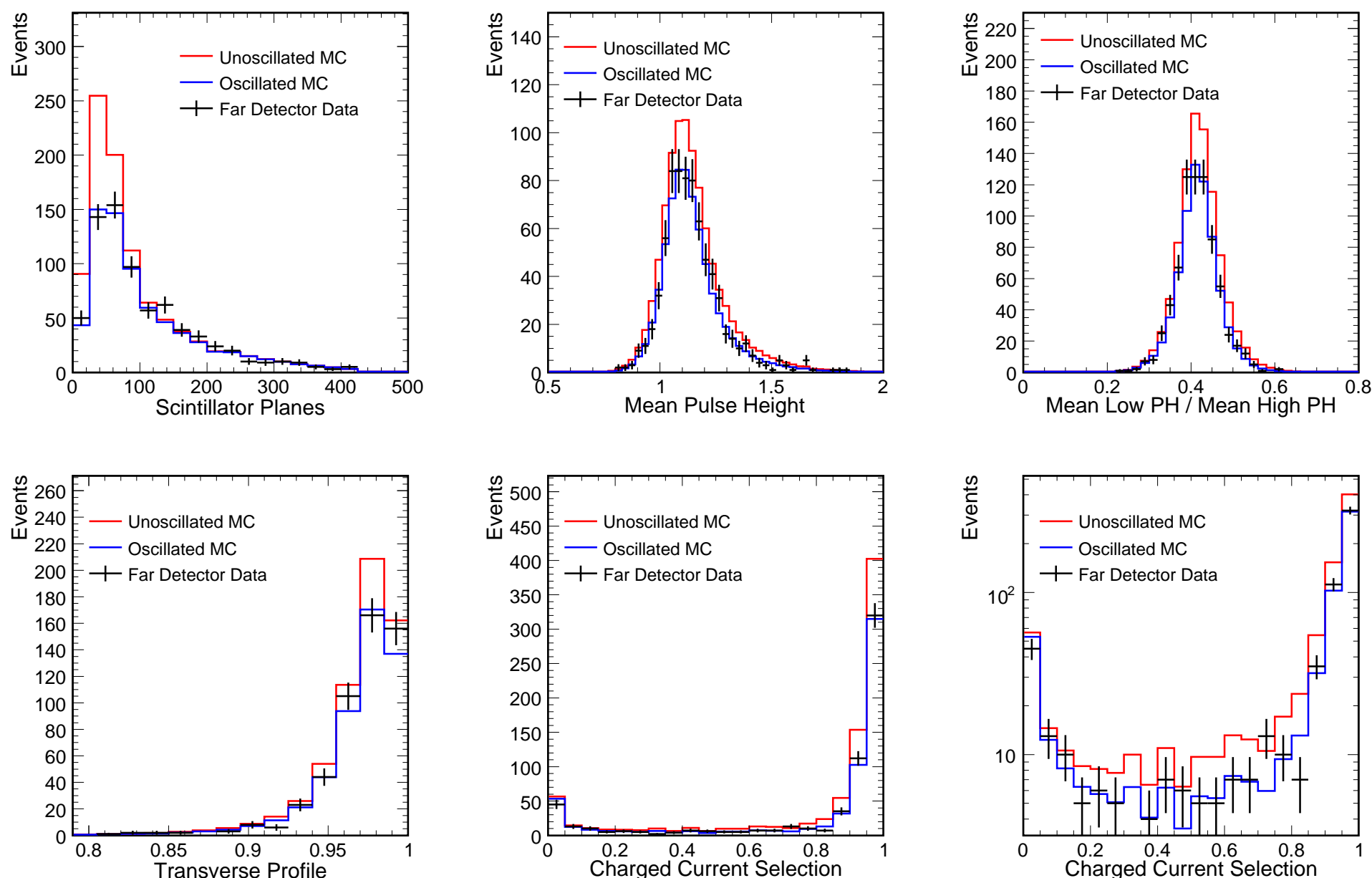

Figure 9.4: Far Detector plots of the CC selection variables for the LE beam. The black points are the data, the red line the unoscillated prediction and the blue the best fit oscillation result. Shown are the CC variables scintillator planes (top left), mean pulse height (top middle), signal fluctuation (top right), transverse profile (bottom right), the CC selector (bottom middle), the $\mathrm{CC}$ selector on a log scale (bottom right). 

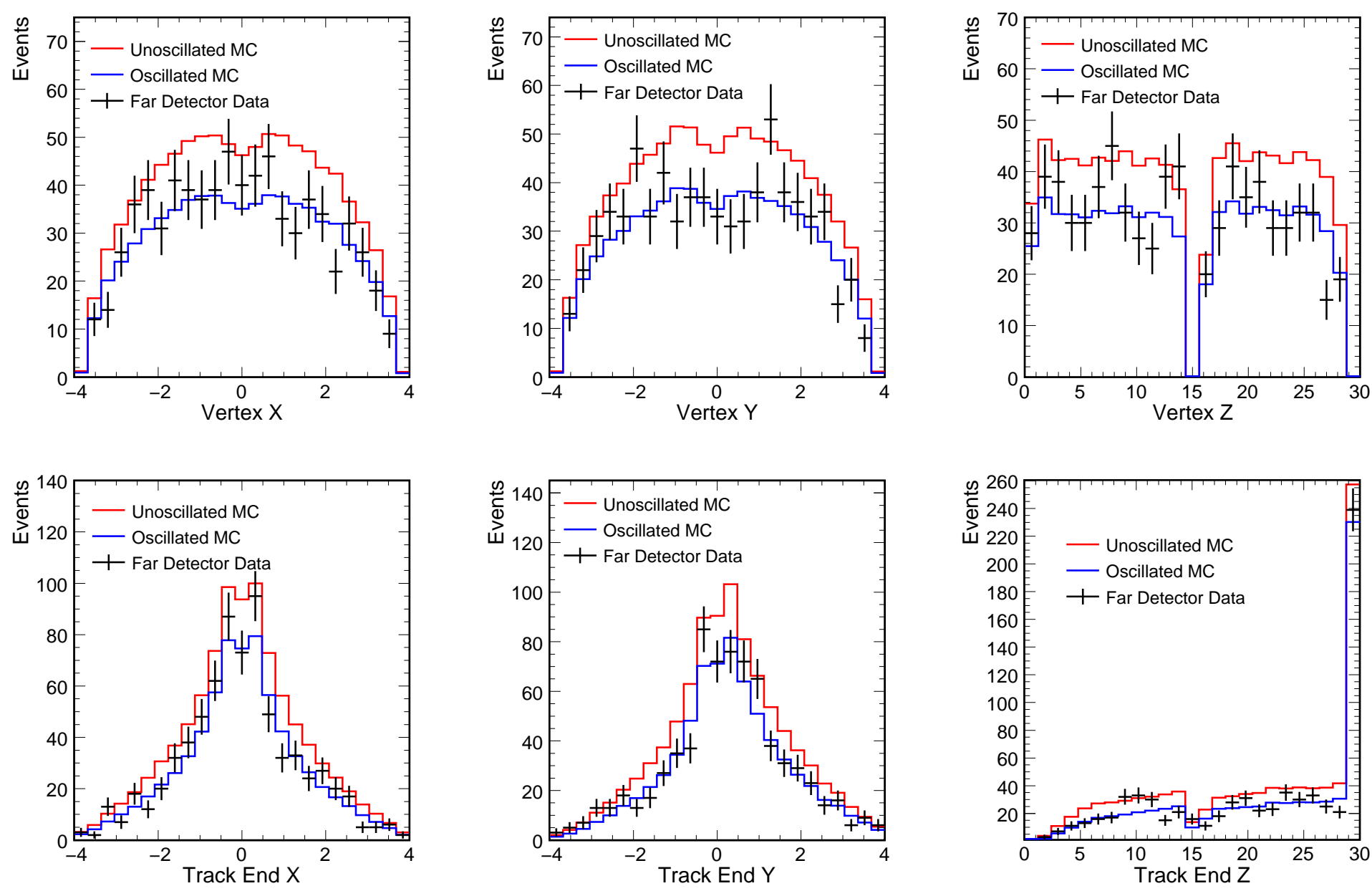

Figure 9.5: Far Detector plots of the event vertex and track ends for the LE beam. The black points are the data, the red line the unoscillated prediction and the blue the best fit oscillation result. Shown are the event vertex position for $x$ (top right), $y$ (top middle), $z$ (top right), and track end positions for $x$ (bottom left), $y$ (bottom middle), and $z$ (bottom right). 

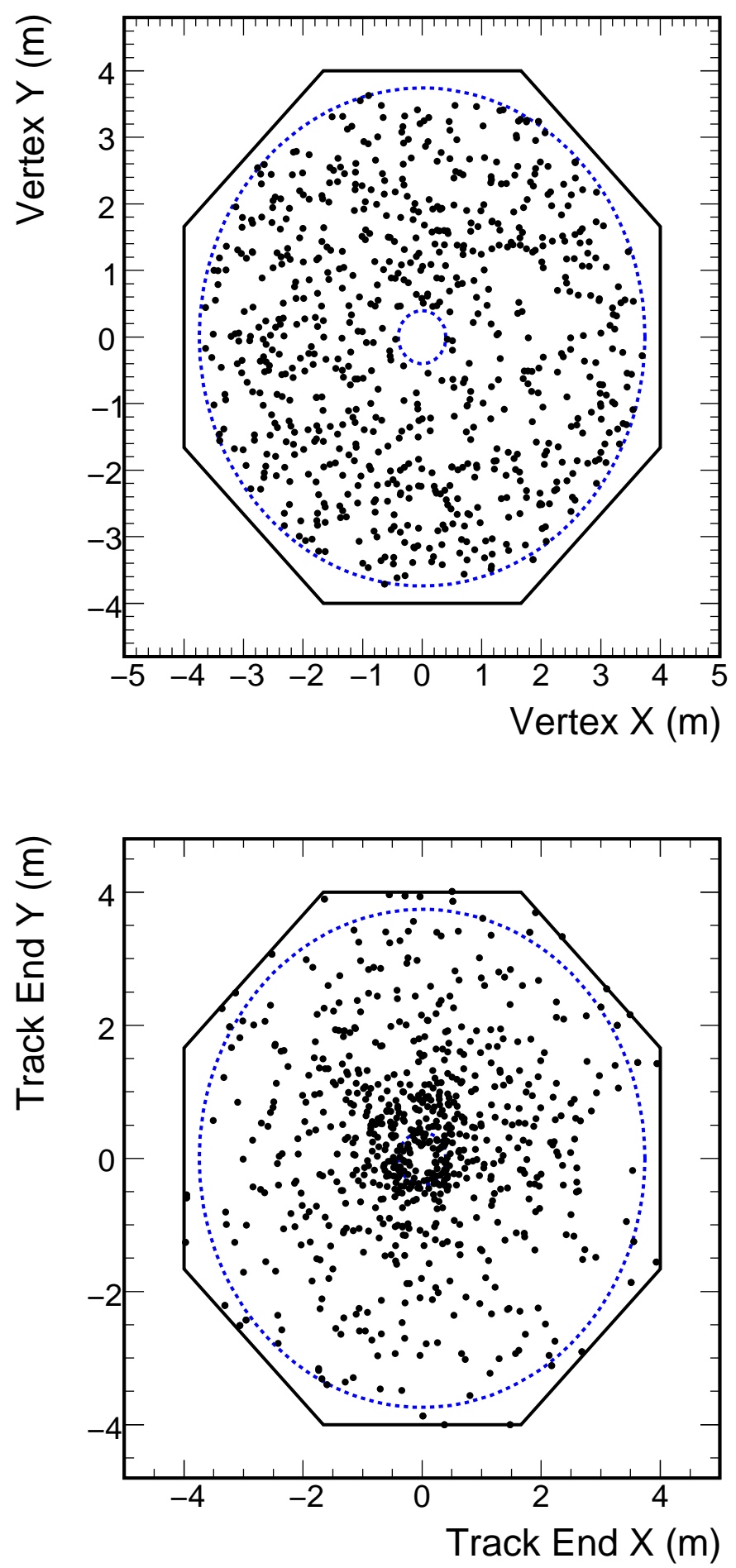

Figure 9.6: 2-D plots in the $x$ and $y$ plane of the event vertex position (top) and the end position of the track (bottom) for the LE beam. The blue line indicates the fiducial volume of the Far Detector. 

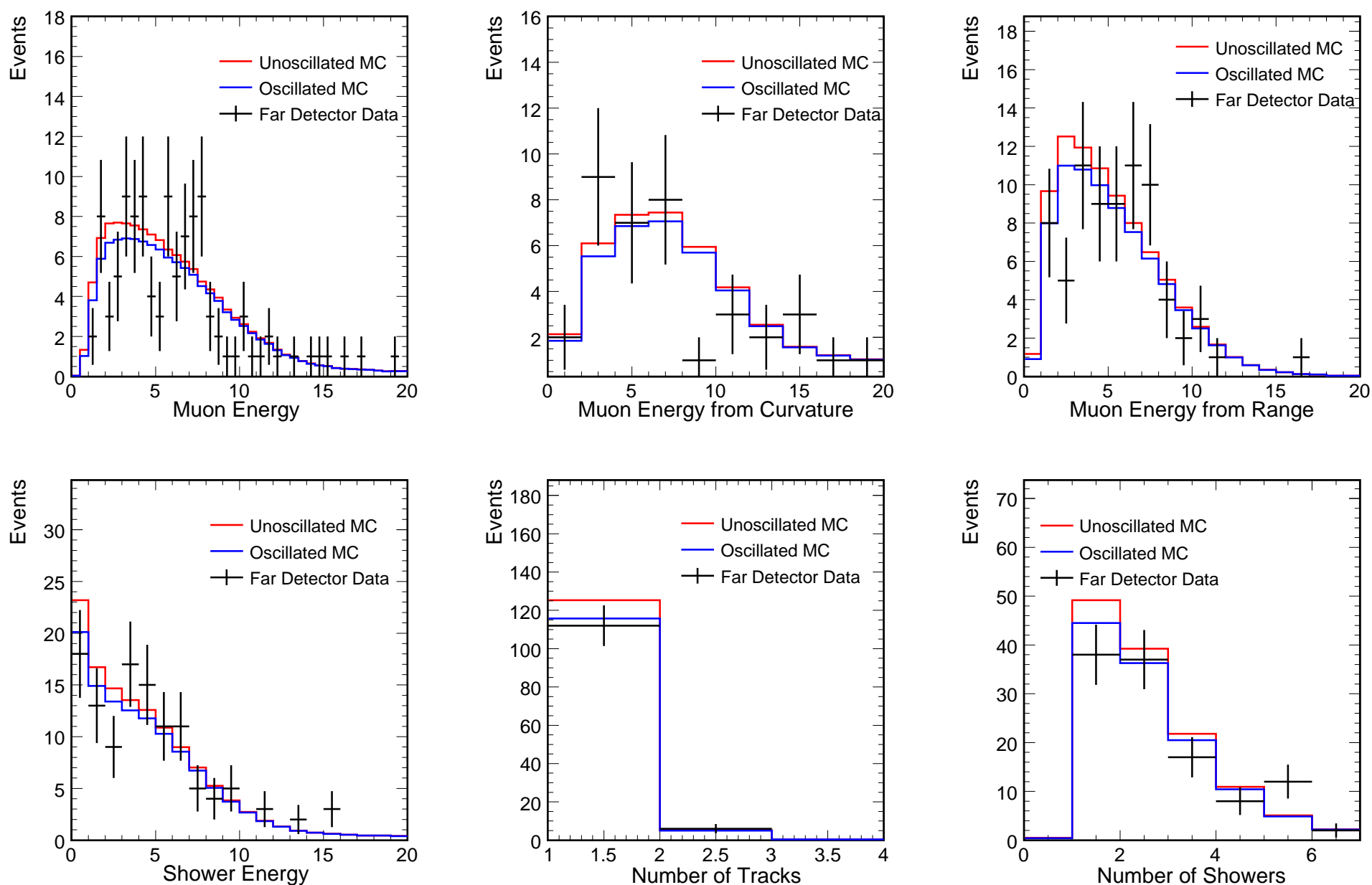

Figure 9.7: Far Detector plots of muon and shower quantities for the HE beam. The black points are the data, the red line the unoscillated prediction and the blue the best fit oscillation result. Shown are the muon energy (top left), the muon energy from exiting tracks (top middle), muon energy from contained tracks (top right), shower energy (bottom left), the number of tracks (bottom middle), the number of showers (bottom right). 

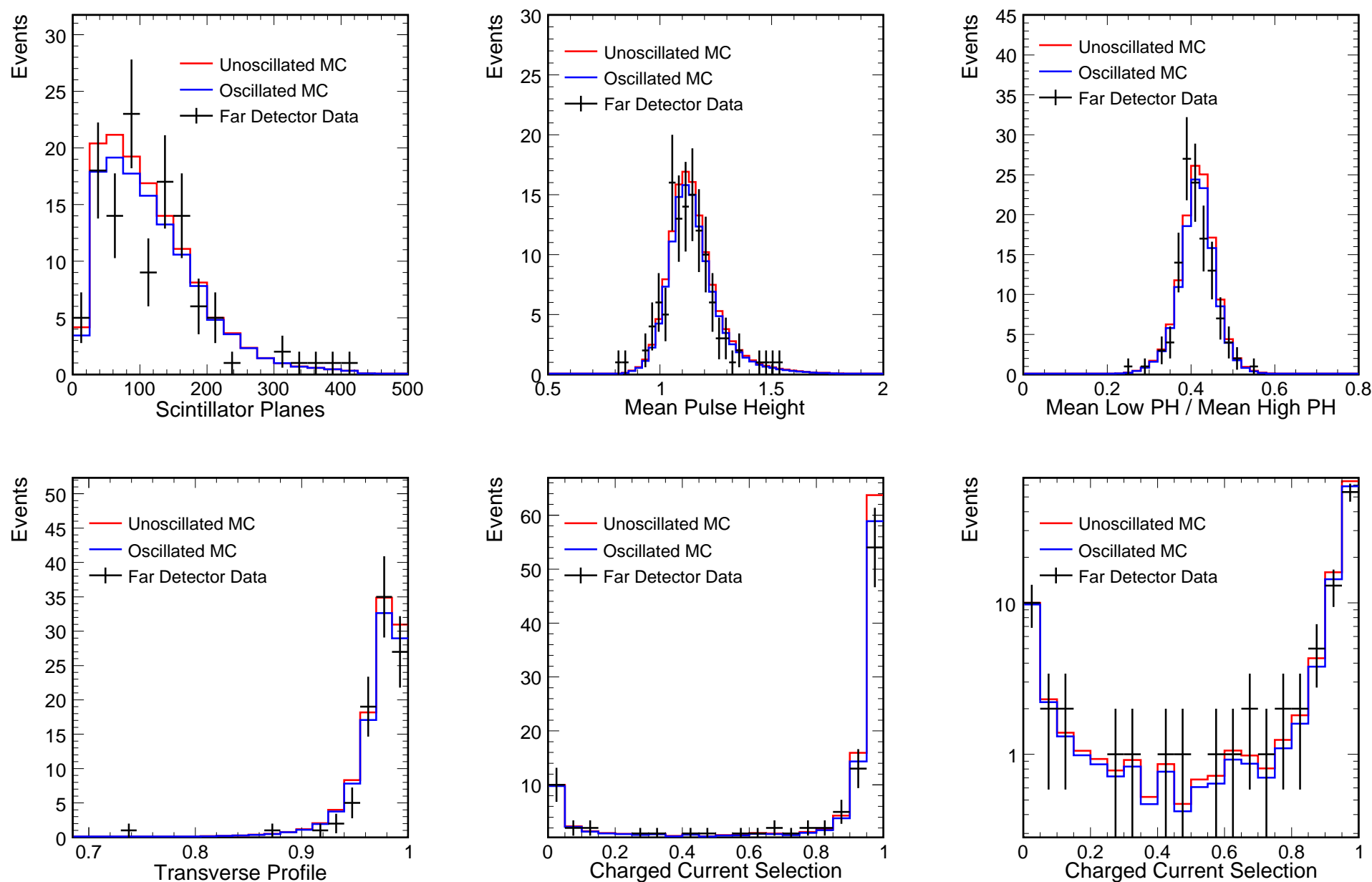

Figure 9.8: Far Detector plots of the charged current selection variables for the HE beam. The black points are the data, the red line the unoscillated prediction and the blue the best fit oscillation result. Shown are the charged current variables scintillator planes (top left), mean pulse height (top middle), signal fluctuation (top right), transverse profile (bottom left), the charged current selection variable (bottom middle), the charged current selection variable on a log scale (bottom right). 

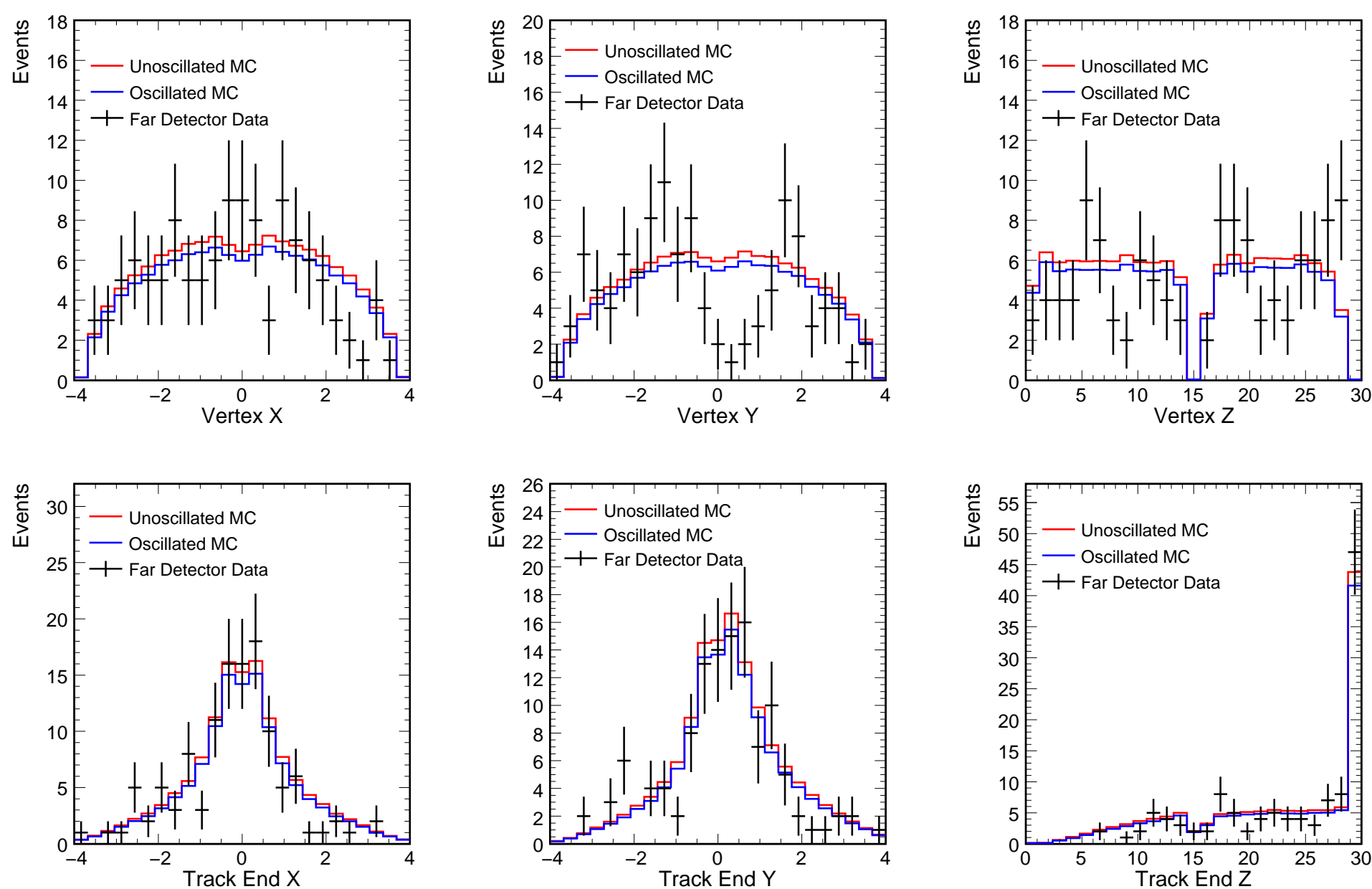

Figure 9.9: Far Detector plots of the event vertex and track ends for the HE beam. The black points are the data, the red line the unoscillated prediction and the blue the best fit oscillation result. Shown are the event vertex position for $x$ (top left), $y$ (top middle), $z$ (top right), and track end positions for $x$ (bottom right), $y$ (bottom middle), and $z$ (bottom right). 


\subsection{Oscillation Analysis}

As given in Chapter 2, the survival probability for a muon neutrino is given by:

$$
P\left(\nu_{\mu}->\nu_{\mu}\right)=1-\sin ^{2}(2 \theta) \sin \left(1.267 \Delta m_{32}^{2} L / E\right) .
$$

To set limits on the oscillation parameters $\Delta m_{32}^{2}$ and $\sin ^{2}\left(2 \theta_{32}\right)$, the above equation is fit to the Far Detector data. The likelihood function is defined as $\chi^{2}=-2 \ln (\lambda)$ where $\lambda$ is defined as [13]:

$$
-2 \ln (\lambda)=\sum_{i}^{\text {bins }} 2\left(N_{i}^{\text {exp }}-N_{i}^{\text {obs }}+2 N_{i}^{\text {obs }} \ln \left(N_{i}^{\text {obs }} / N_{k}^{\text {exp }}\right)\right)+\sum_{j=1}^{\text {systematics }}\left(\frac{\Delta \alpha_{j}}{\sigma_{\alpha_{j}}}\right)^{2}
$$

where $N_{i}^{o b s}$ and $N_{i}^{e x p}$ are the observed and expected number of events in bin $i$, and $\alpha_{j}$ are the systematic parameters included in the fit. The data was binned into $0.5 \mathrm{GeV}$ bins up to $10 \mathrm{GeV}, 1 \mathrm{GeV}$ bins from $10-20 \mathrm{GeV}$ and three more bins from 20-30 GeV, 30-50 $\mathrm{GeV}$, and 50-200 GeV.

Figures 9.11, 9.12, and 9.13 show the $\nu_{\mu}$ energy spectrum for the Far Detector data compared with the unoscillated prediction and the best fit prediction. The best fit prediction came from a simultaneous fit to data from all three beam configurations.

Ignoring for the moment the effect of systematic errors, the best fit points for the oscillation parameters were found at $\Delta m_{32}^{2}=2.44_{-0.11}^{+0.11} \times 10^{-3} \mathrm{eV}^{2}$ and $\sin ^{2}\left(2 \theta_{32}\right)=$ $1.00_{-0.04}^{+0.00}$. The result had a $\chi^{2}=91.35$ for 97 degrees of freedom. Table 9.3 lists the contribution to the $\chi^{2}$ from each data set. Figure 9.14 shows the $68 \%$ and $90 \%$ C.L. assuming gaussian statistics defined by contours of $\Delta \chi^{2}=2.31$ and 4.61 respectively. If the physical boundary for $\sin ^{2}\left(2 \theta_{32}\right)$ is relaxed, the results are $\sin ^{2}\left(2 \theta_{32}\right)=1.09_{-0.09}^{+0.07}$ and $\Delta m_{32}^{2}=2.31_{-0.15}^{+0.17} \times 10^{-3} \mathrm{eV}^{2}$.

The official MINOS results obtained a slightly lower value of $\Delta m_{32}^{2}=2.38 \times 10^{-3} \mathrm{eV}^{2}$ and $\sin ^{2}\left(2 \theta_{32}\right)=1$ without systematics. The results of this analysis are consistent with that measurement. Part of the difference between the two methods is a consequence of 

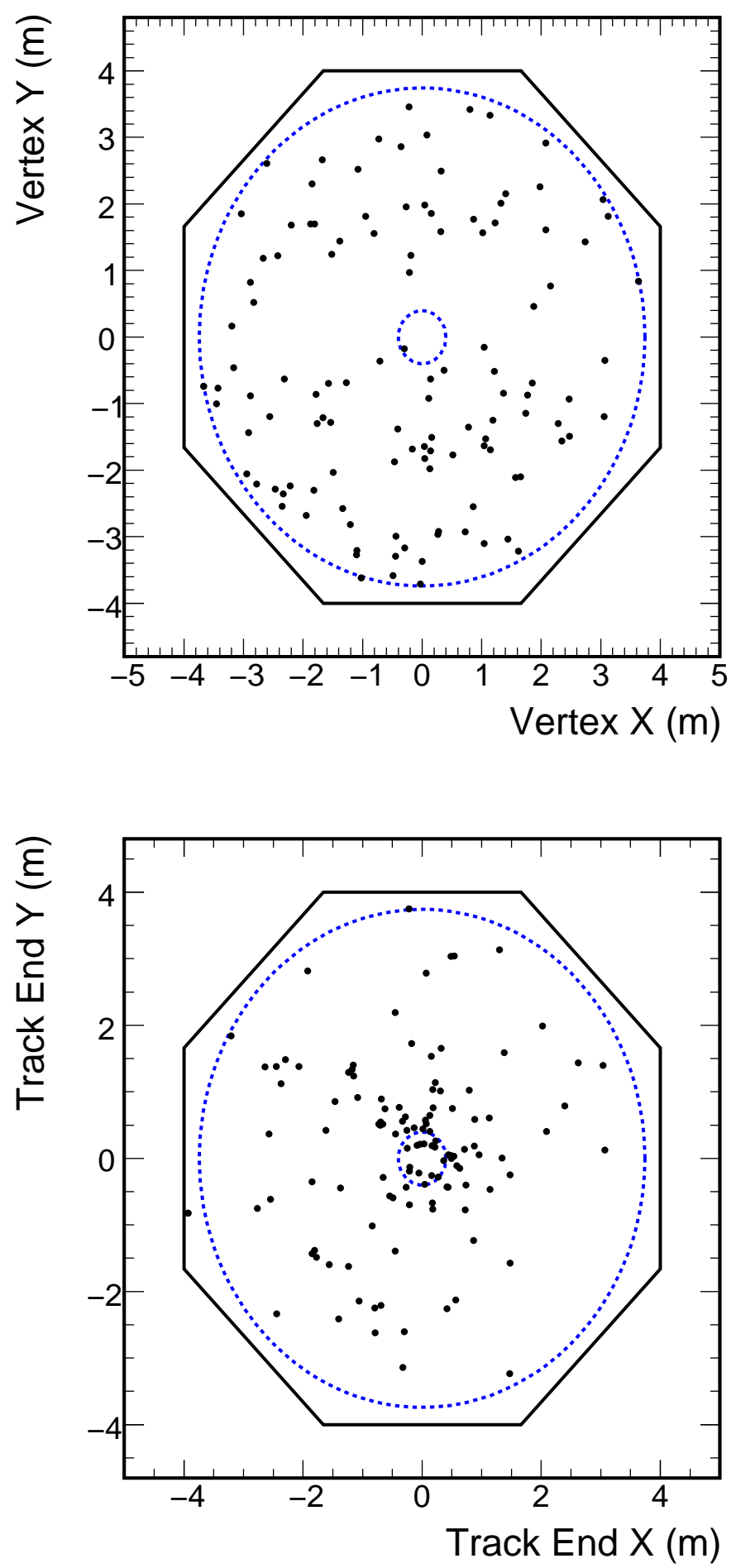

Figure 9.10: 2-D plots in the $x$ and $y$ plane of the event vertex position (top) and the end position of the track (bottom) for the HE beam. The blue line indicates the fiducial volume of the Far Detector. 


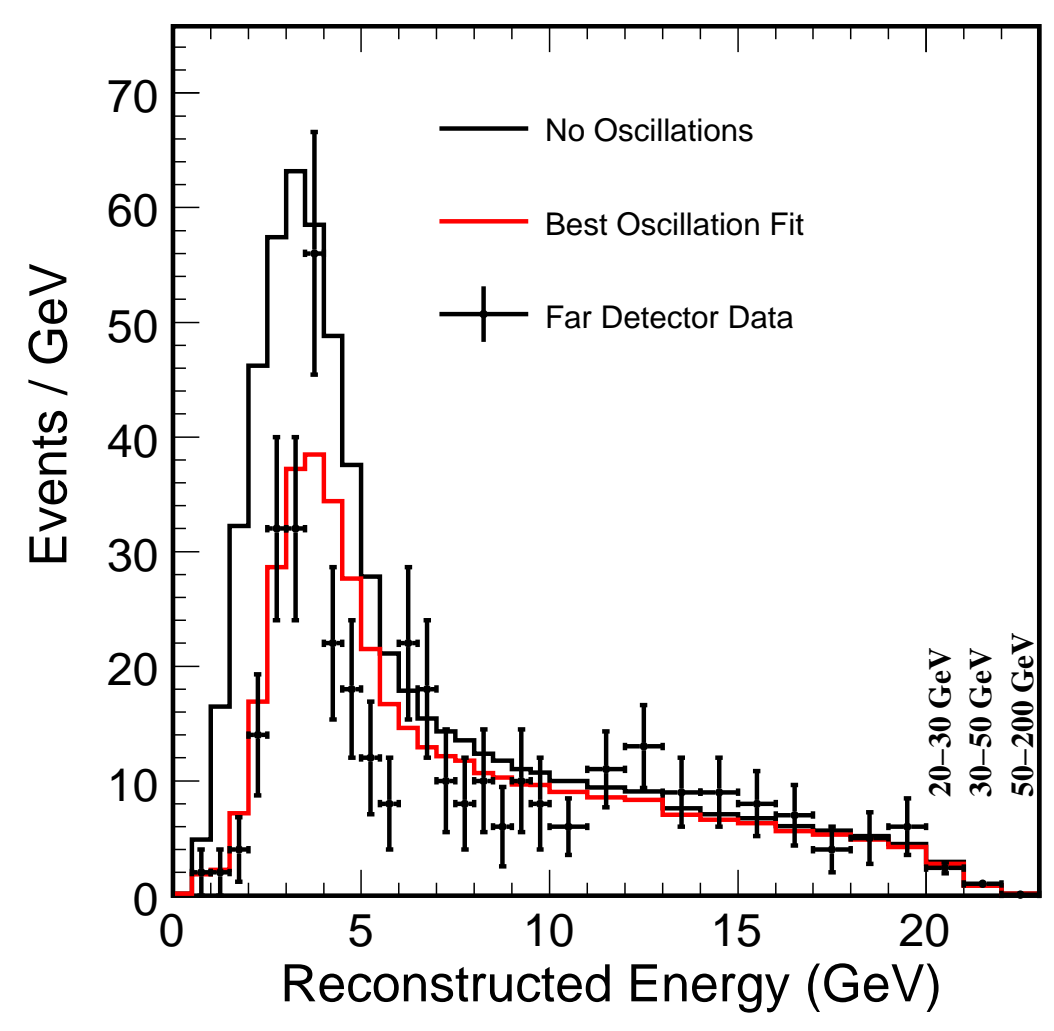

Figure 9.11: Neutrino energy spectrum from the LE Run1 configuration compared to the best fit prediction to all three beams simultaneously. 


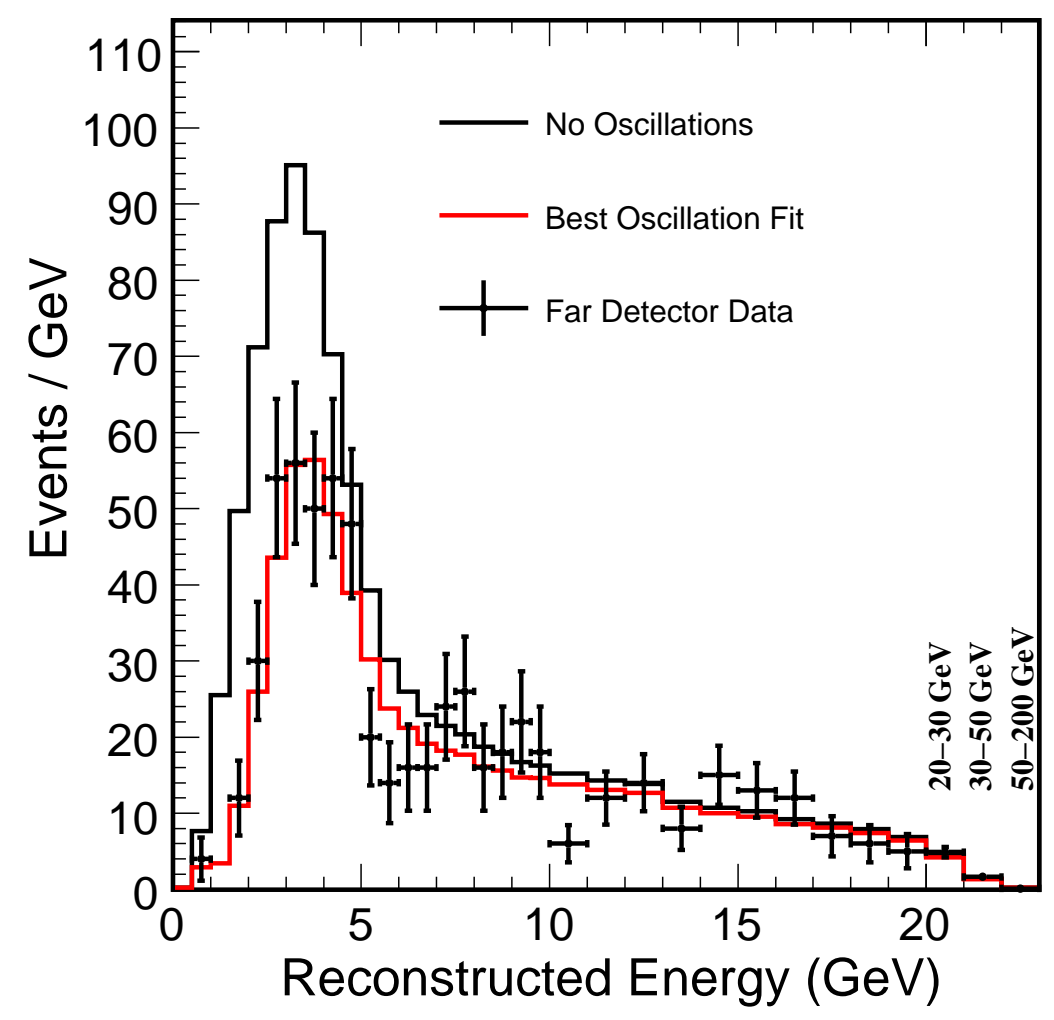

Figure 9.12: Neutrino energy spectrum from the LE Run2 configuration compared to the best fit prediction to all three beams simultaneously. 


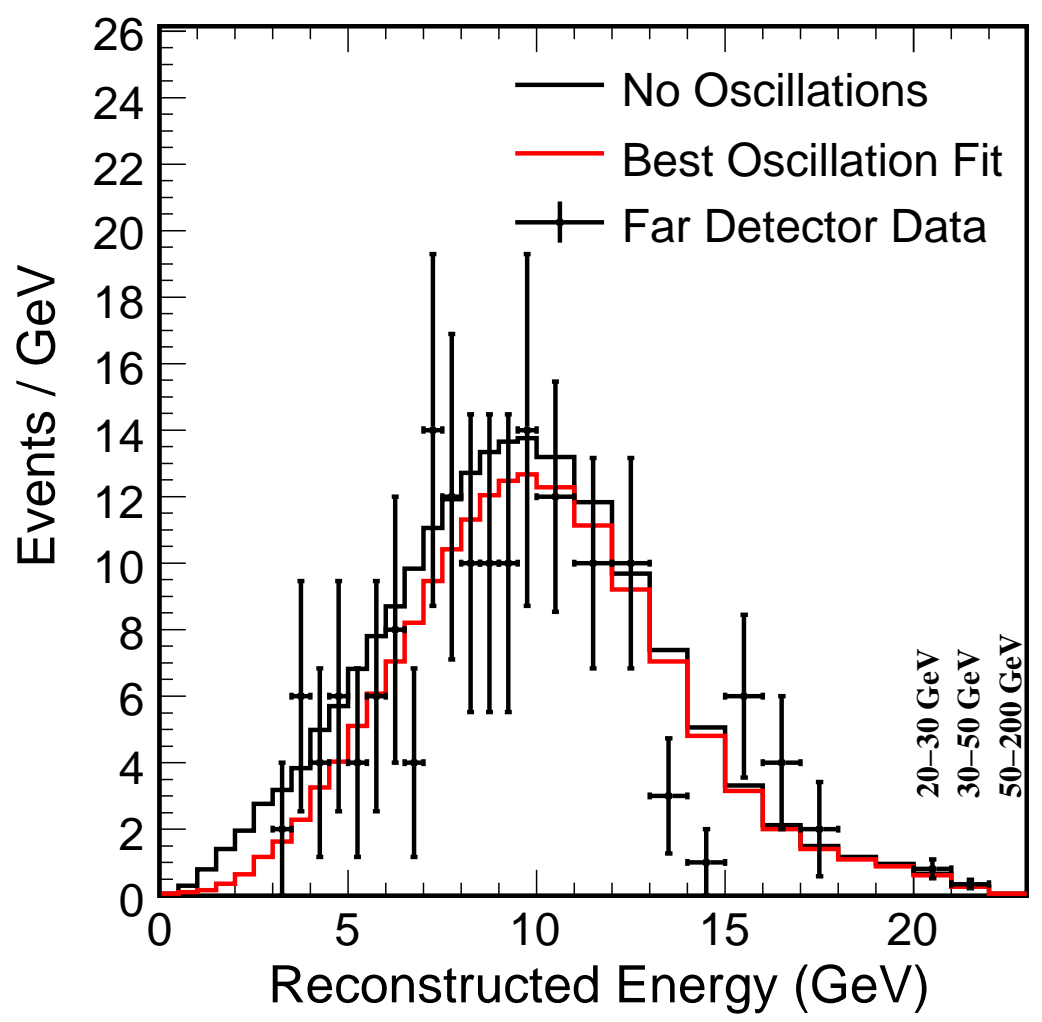

Figure 9.13: Neutrino energy spectrum from the HE configuration compared to the best fit prediction to all three beams simultaneously. 


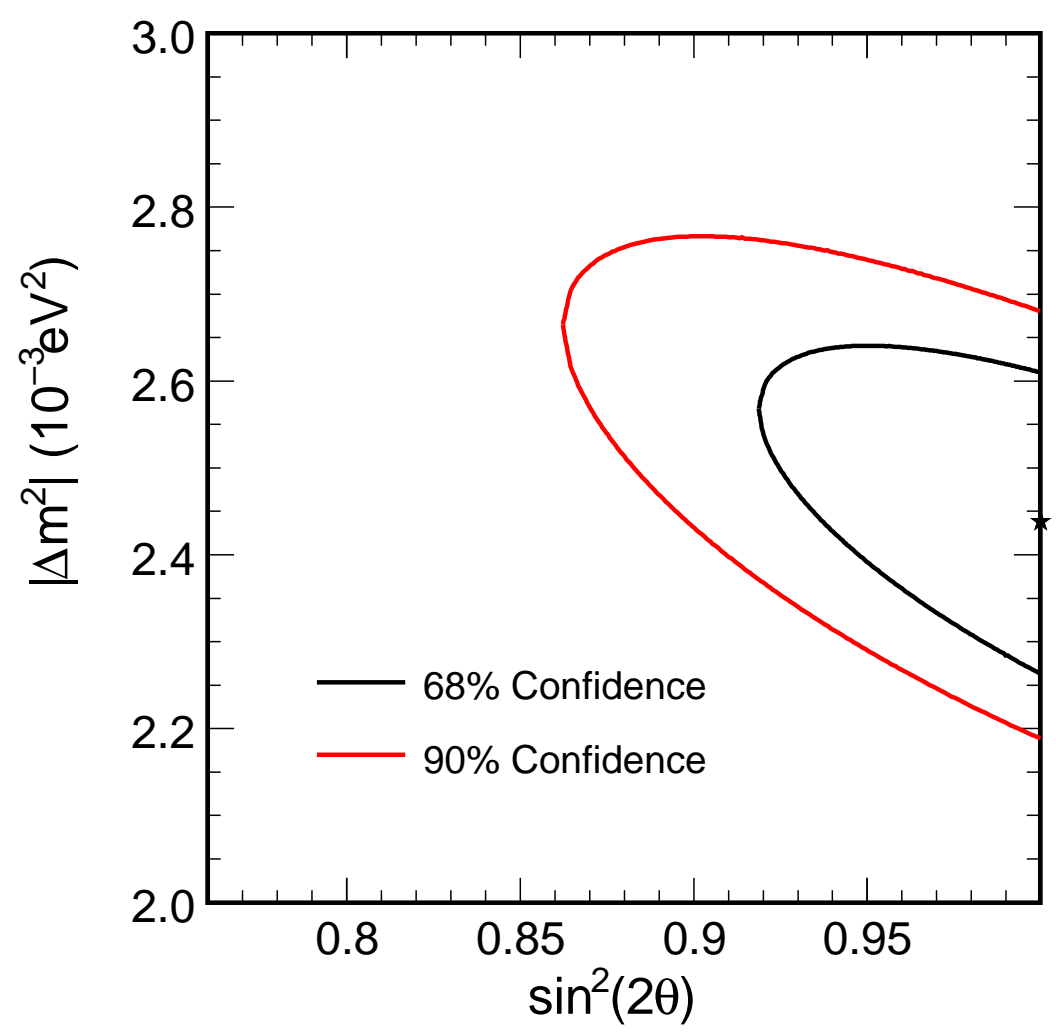

Figure 9.14: Confidence limits of $68 \%$ (red) and 90\% (black) from fitting the LE RunI, LE RunII and HE RunII simultaneously. 


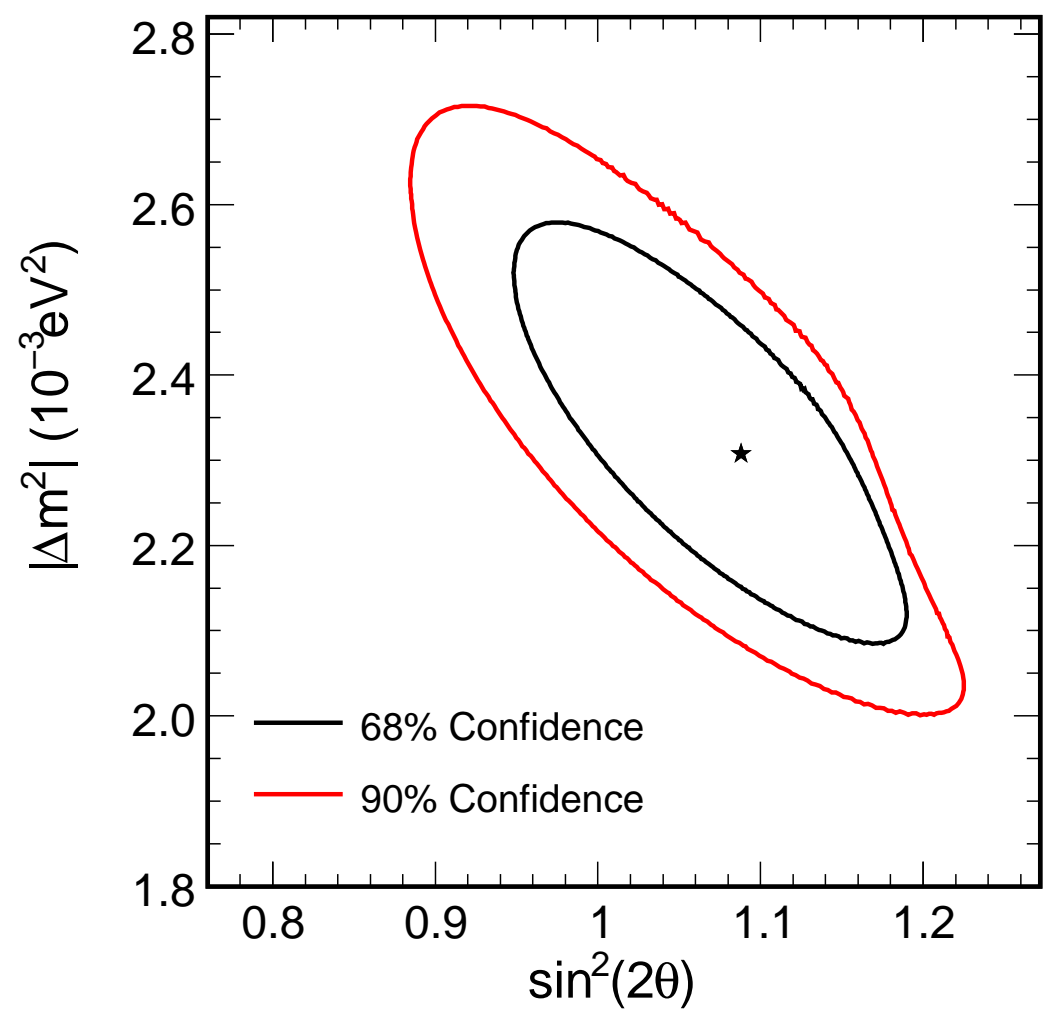

Figure 9.15: Confidence limits of $68 \%$ (red) and 90\% (black) from fitting the LE RunI, LE RunII and HE RunII simultaneously where $\sin ^{2}\left(2 \theta_{32}\right)$ is unconstrained. 


\begin{tabular}{lcc}
\hline \hline Data Set & $\chi^{2}$ Contribution & Degrees of Freedom \\
\hline LE Run1 & 28.96 & 33 \\
LE Run2 & 33.81 & 33 \\
HE & 28.58 & 33 \\
\hline Total & 91.35 & 97 (99-2 fit parameters) \\
\hline \hline
\end{tabular}

Table 9.3: The contribution to the $\chi^{2}$ and degrees of freedom from each data set.
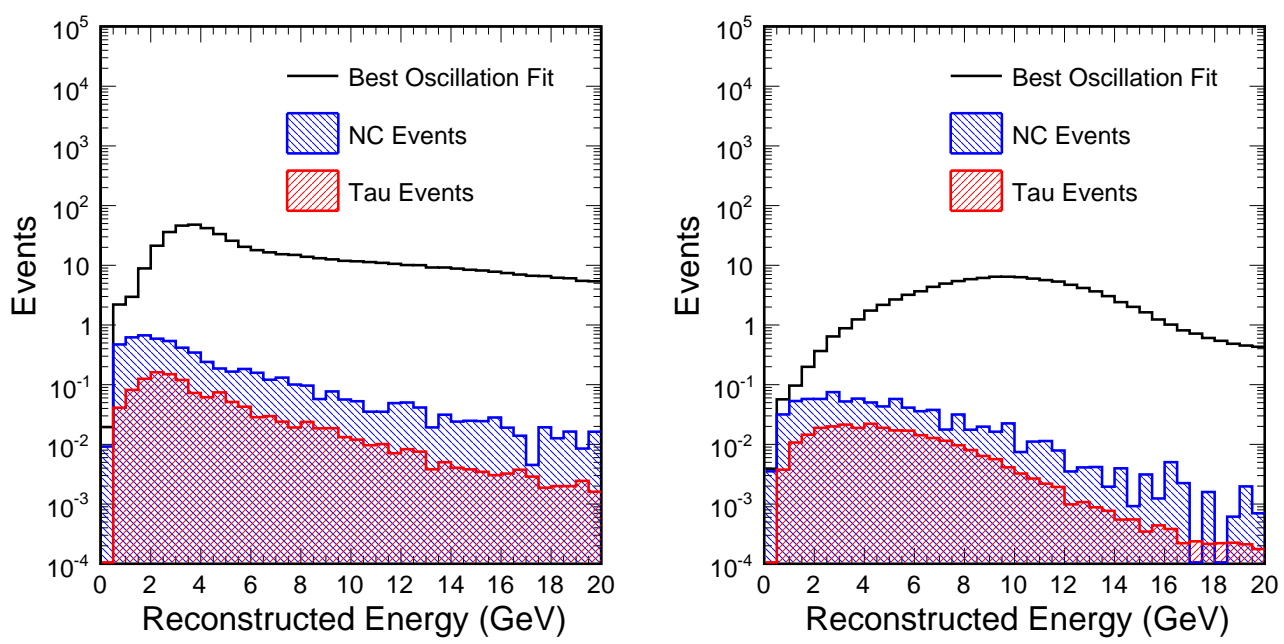

Figure 9.16: The background components from $\nu_{\tau}$ and neutral current events for the LE beam (left) and HE beam (right).

the different extrapolation methods. The extrapolation method used in this thesis applies corrections to the muon energy, shower energy and the neutral current background. If these corrections are ignored the results are the same as that of the primary analysis.

Figure 9.16 shows the background component from $\mathrm{NC}$ events and $\nu_{\tau}$ events. The total number of events expected from neutral current events is 5.8 and from tau events 1.3 in LE configuration.

\subsubsection{Fit to Individual Runs}

Each different beam configuration can be fit independently for the oscillation parameters.

Table 9.4 shows the results of these fits and Figure 9.17 compares the sensitivities. 


\begin{tabular}{lcc}
\hline \hline Data Set & $\begin{array}{c}\Delta m_{32}^{2} \\
\left(10^{-3} \mathrm{eV}^{2}\right)\end{array}$ & $\sin ^{2}\left(2 \theta_{32}\right)$ \\
\hline LE Run1 Only & 2.59 & 1.00 \\
LE Run2 Only & 2.35 & 1.00 \\
HE Only & 2.48 & 1.00 \\
LE Run1 Only (Unconstrained) & 2.52 & 1.04 \\
LE Run2 Only (Unconstrained) & 2.17 & 1.14 \\
HE Only (Unconstrained) & - & - \\
LE + HE & 2.44 & 1.00 \\
LE + HE (Unconstrained) & 2.28 & 1.06 \\
\hline \hline
\end{tabular}

Table 9.4: The best fit oscillation parameters when fitting each data run alone. Unconstrained fits allow $\sin ^{2}\left(2 \theta_{32}\right)$ to be larger than 1 . The unconstrained HE fit did not have enough statistics to converge in the fit.

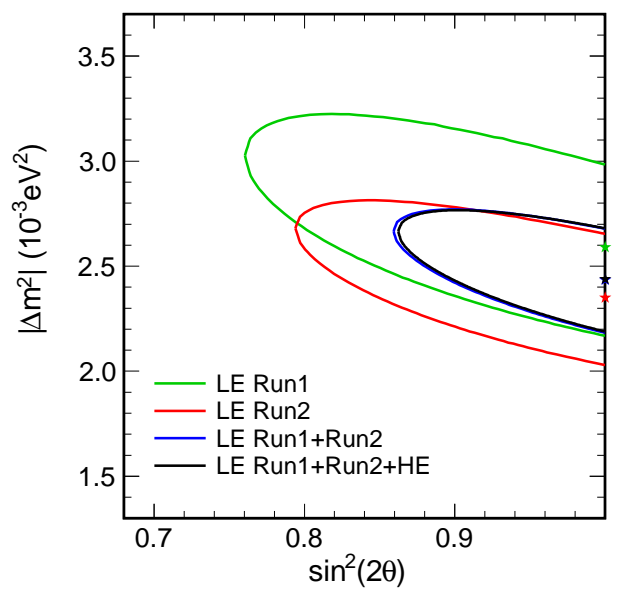

Figure 9.17: The 90\% C.L. for individual runs: LE Run1 (green), LE RunII (red), combined LE runs (blue), combined LE+HE runs (black). The stars show the best fit points. 

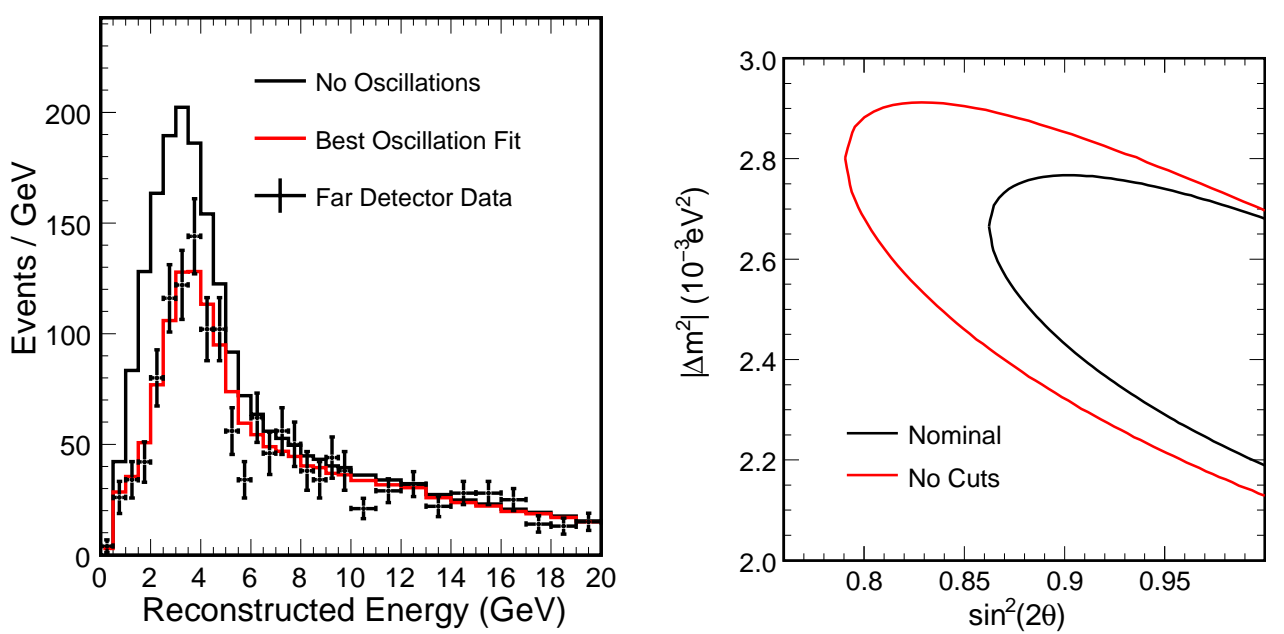

Figure 9.18: The effect of not including any cuts on the event selection. Left: The best fit spectrum to the oscillation parameters. Right: The $90 \%$ C.L. for both the nominal case and when cuts are applied.

\subsubsection{Fitting the Data with No Cuts}

To test the impact of the selection cuts, the oscillation parameters are found without applying the neutral current and charge sign selection cuts. In this case, it does not make sense for the extrapolation to apply the $\mathrm{NC}$ correction since the fit in the Near Detector was only done for CC-like events. The results are shown in Figure 9.18 with best fit values of $\Delta m_{32}^{2}=2.42 \times 10^{-3} \mathrm{eV}^{2}, \sin ^{2}\left(2 \theta_{32}\right)=1$. As expected, the sensitivity is degraded due to the increased background, but the central values agree.

\subsubsection{Fitting with Systematics}

The three largest systematic errors found in Chapter 8 were those coming from relative uncertainties between the Near and Far Detectors: the relative shower energy, the residual ND difference, and the normalization uncertainty. These were treated as nuisance parameters and Figure 9.19 shows the 90\% C.L. 


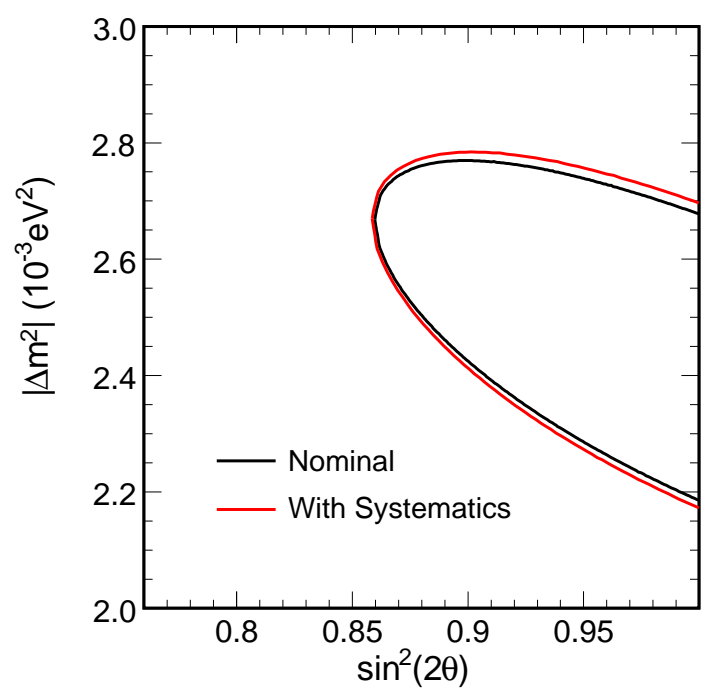

Figure 9.19: A comparison of the 90\% C.L. when the largest systematic errors are included as nuisance parameters (red) and with no systematics (black).

\subsection{Comparison with Published MINOS Results}

The MINOS collaboration published oscillation results of the same data analyzed here in [48]. This analysis served as a cross-check to that of the main extrapolation procedure, the Beam Matrix method (see Section 6.2). A comparison of the contours is shown in Figure 9.20. Table 9.5 also lists other results that have been released of the MINOS data. The analysis presented here agrees well with the published results. The constraints from this analysis are better because of the impact of the global errors is reduced by fitting for these parameters in the Near Detector fit.

\subsection{Dip at $5 \mathrm{GeV}$}

One area where the data does not agree with the oscillated prediction is at falling edge of the peak at 5-6 GeV. A careful examination of Figures 9.11 and 9.12 for RunI and RunII show that both have a deficit in this region. Figure 9.21 combines the events from RunI and RunII showing the total deficit in the 5-6 GeV bins. The predicted number events in 


\begin{tabular}{l|cc}
\hline \hline & $\begin{array}{c}\Delta m_{32}^{2} \\
\left(10^{-3} \mathrm{eV}^{2}\right)\end{array}$ & $\sin ^{2}\left(2 \theta_{32}\right)$ \\
\hline Published Result (2006) [80] & $2.74_{-0.20}^{+0.44}$ & $1.00_{-0.13}$ \\
Preliminary Result (2007) [81] & $2.38_{-0.16}^{+0.20}$ & $1.00_{-0.08}$ \\
Published Result (2008) [48] & $2.44 \pm 0.13$ & $1.00_{-0.05}$ \\
This Analysis & $2.45 \pm 0.12$ & $1.00_{-0.04}$ \\
\hline \hline
\end{tabular}

Table 9.5: A comparison of the best fit oscillation parameters from the results of this thesis with the other MINOS results.

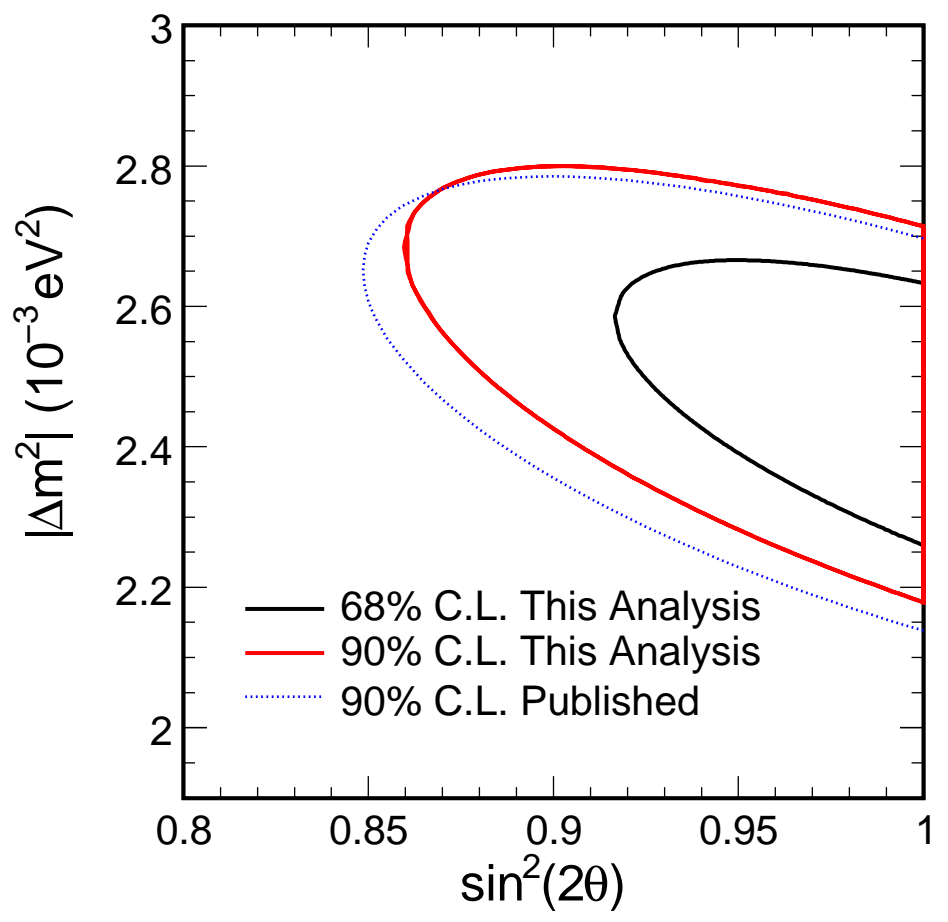

Figure 9.20: Comparison of the results from this thesis with the recently published MINOS results [48]. 


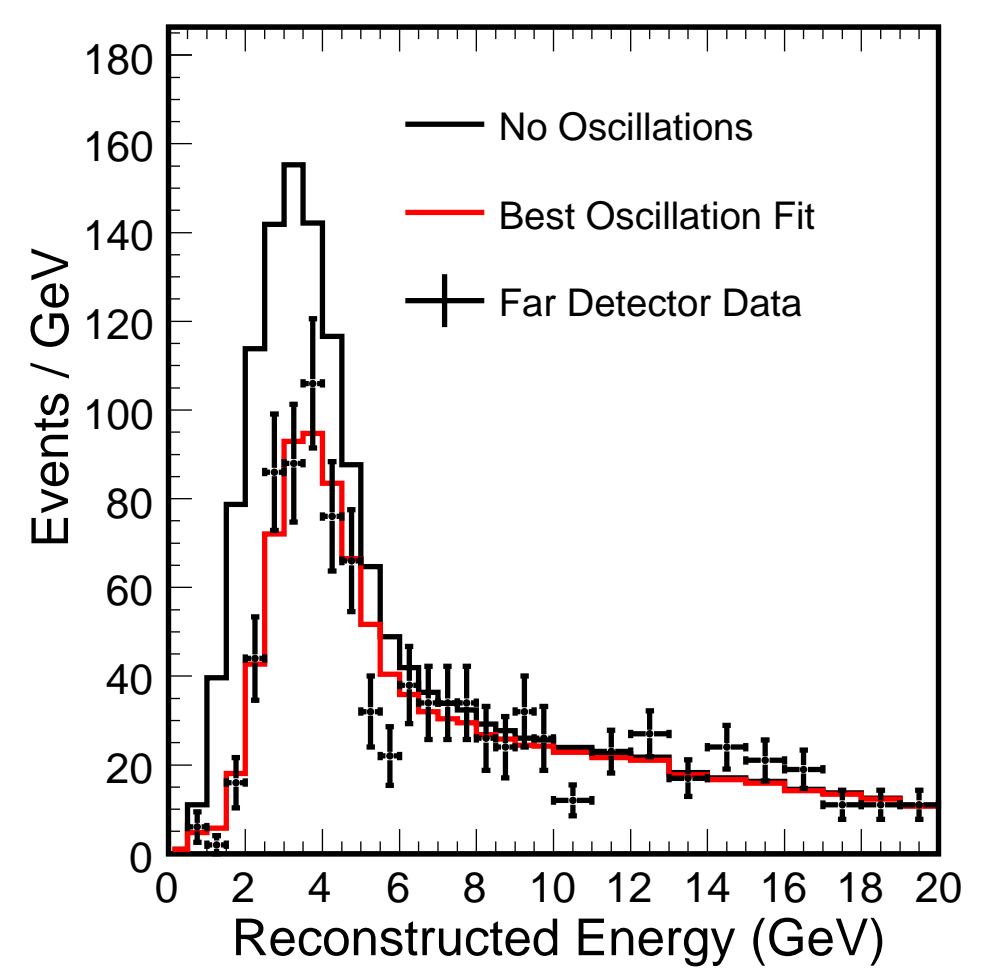

Figure 9.21: The LE RunI+RunII combined neutrino energy spectrum. A $\sim 3 \sigma$ deficit is seen in the $5-6 \mathrm{GeV}$ bins.

the two bins is 46 while the data shows only 27 , a $2.8 \sigma$ effect. This could be a statistical fluctuation or it could be a sign of an unaccounted for systematic error. Comparison with Figure 3.13 shows that this is also the same region where the uncertainty from alignment and focusing is the largest.

\subsubsection{Cut Relaxation}

It is important to see if events are being removed by the event selection cuts in the 5-6 GeV range. The two most likely cuts to affect this region are the charge sign cut and the CC selection cut. Figure 9.22 shows the ratio of the neutrino energy spectrum before and after relaxing each cut. The charged current selection cut does remove more events in the $5-6 \mathrm{GeV}$ bin for the data than expected from the Monte Carlo. The charge sign cut shows good agreement. 

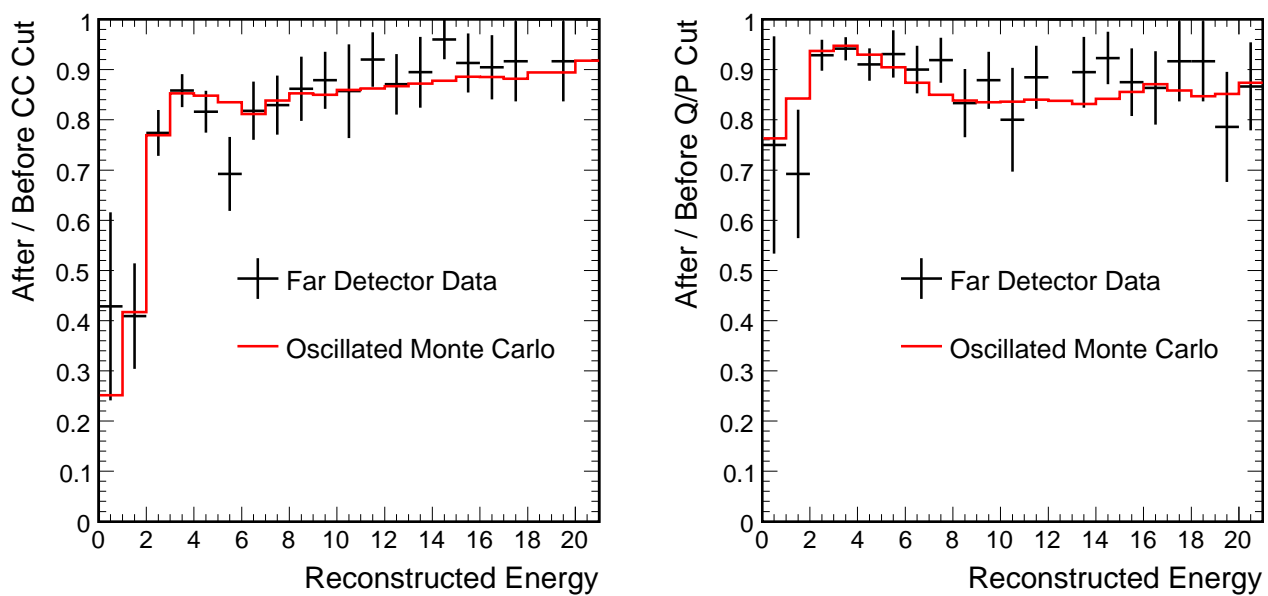

Figure 9.22: Left: The ratio of the data and best fit oscillated Monte Carlo spectrum before and after the charged current selection cut. Right: The ratio of the data and best fit oscillated Monte Carlo spectrum before and after the charge sign cut.

\subsubsection{Different Regions of $y$}

If there were a $y$ dependence on the events that are missing that may provide clues as to the cause of the dip. Figure 9.23 shows the $\nu_{\mu}$ energy spectrum for different regions of $y$. For high $y$ values there is no deficit in the $5-6 \mathrm{GeV}$ range, but the low $y$ regions show an even more enhanced deficit.

\subsubsection{Observables between $3-7 \mathrm{GeV}$}

It is important to check other reconstructed quantities in the region of interest. Figure 9.3 already showed good agreement over all energy regions, but that may not be true for 5-6 $\mathrm{GeV}$ region. To increase statistics an energy range was chosen to from 3-7 GeV. Several quantities are examined in Figure 9.24. 

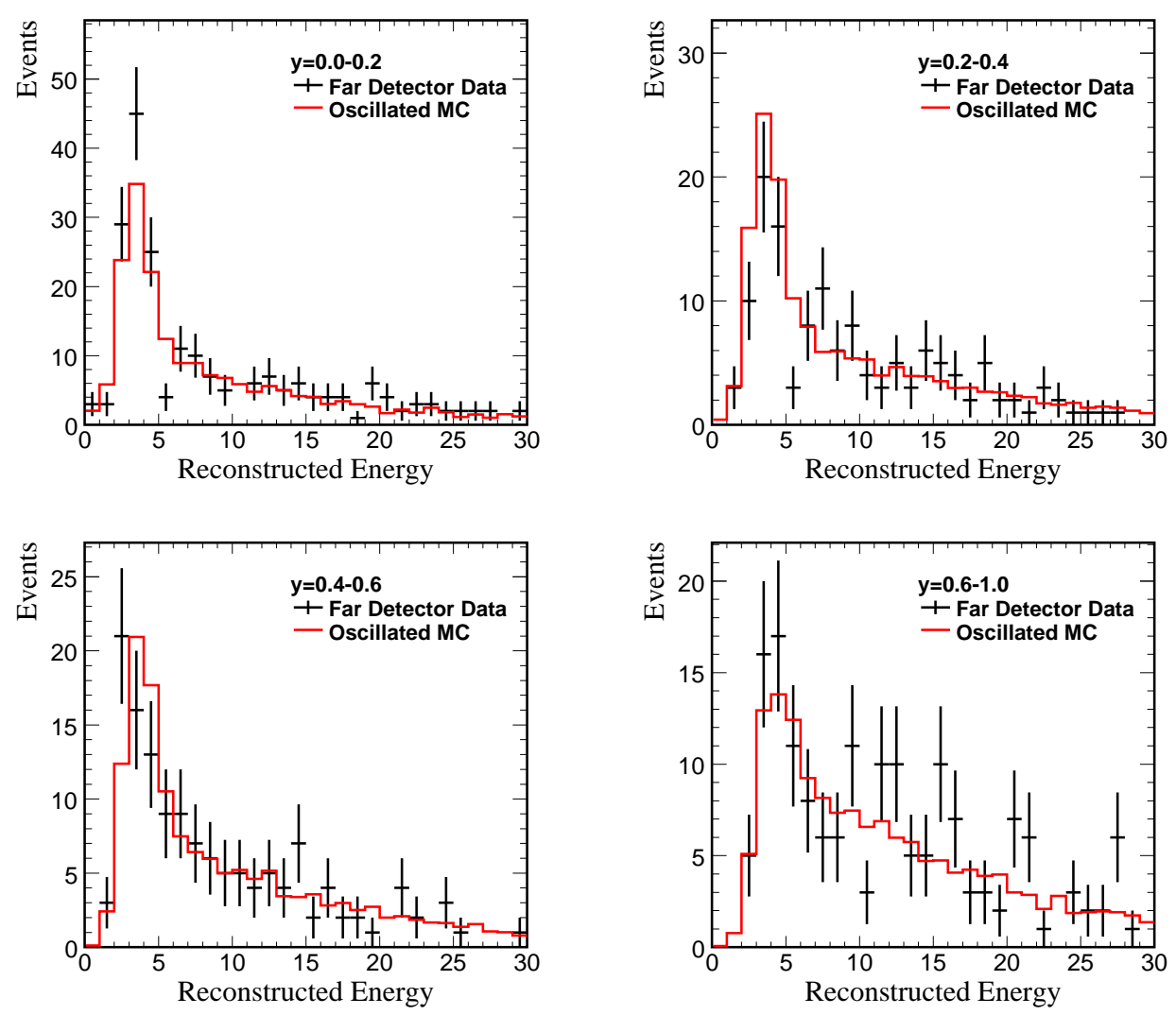

Figure 9.23: The Far Detector LE neutrino energy spectrum for data and the best fit prediction for different regions of $y$ : $y<0.2$ (top right), $0.2<y<0.4$ (top left), $0.4<y<0.6$ (bottom right), $0.6<y<1$ (bottom left). 

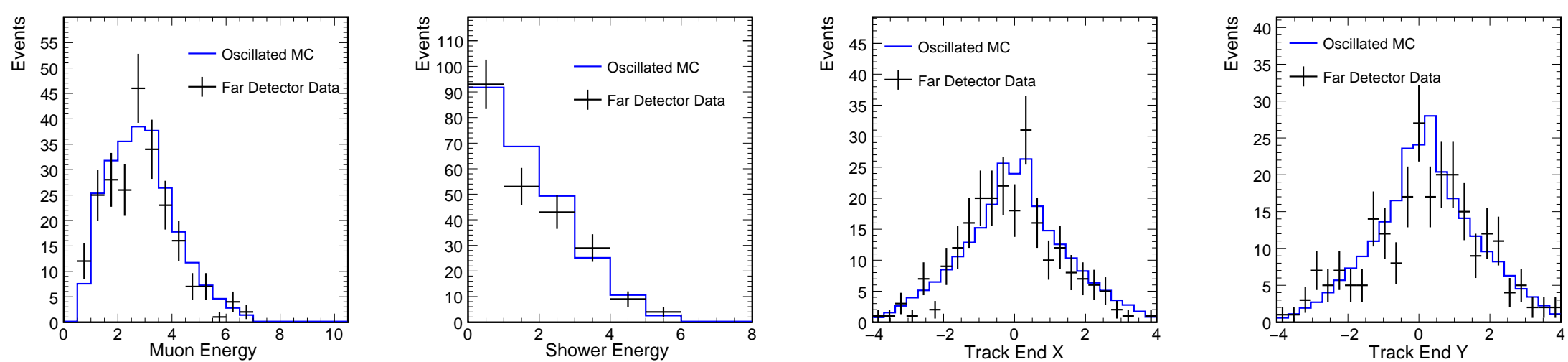

0
0
$\ddots$
$\ddots$
0
0
0
0
0
$<$
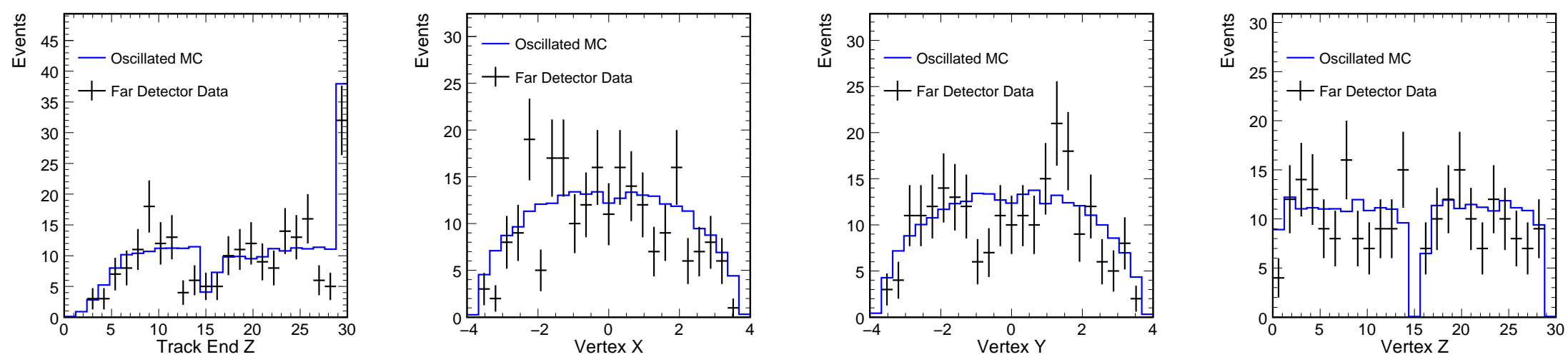

Figure 9.24: Various quantities in the Far Detector for neutrino energies from 3-7 GeV. 
Among these plots, there is only one that may be cause for concern. The plot showing the $z$ position of the end of the track shows a deficit at the end of both supermodules.

\subsubsection{Focusing Effects}

Since the largest region of uncertainty for focusing is in the falling edge of the peak, a study was done to see if an incorrect account of the focusing could cause of the deficit. To test this hypothesis two additional parameters were introduced in the fit to the Far Detector: the calibration of the horn current and the modeling of the current or the effect of the skin depth.

Figure 9.25 shows the results of these fits. The best fit value for the horn current was $-3.77 \%$, and the significance of the dip was reduced to $2.2 \sigma$. The result of the skin depth was a best fit value of $-4.13 \sigma$, where $1 \sigma$ is equal to a skin depth of $6 \mathrm{~mm}$. In this case the significance of the dip was reduced to $1.7 \sigma$. Both cases can be ruled out by measurements of the beamline instrumentation. A further point, is that because of detector resolution, a $1 \mathrm{GeV}$ wide deficit in reconstructed neutrino energy must be even narrower in true neutrino energy. This evidence makes focusing effects an unlikely candidate to explain the discrepancy.

\subsubsection{Summary of $5 \mathrm{GeV}$ dip}

While studying the deficit in the $5-6 \mathrm{GeV}$ bin several issues have arisen:

i. The charged current selection cut removes more events in that bin than in surrounding bins. The cut removes 12 out of 39 events in that bin and only 16 out of 87 in the bin before and 8 out of 43 in the bin after.

ii. The deficit is only observed for low $y$ events, no deficit is seen above $y=0.4$. 

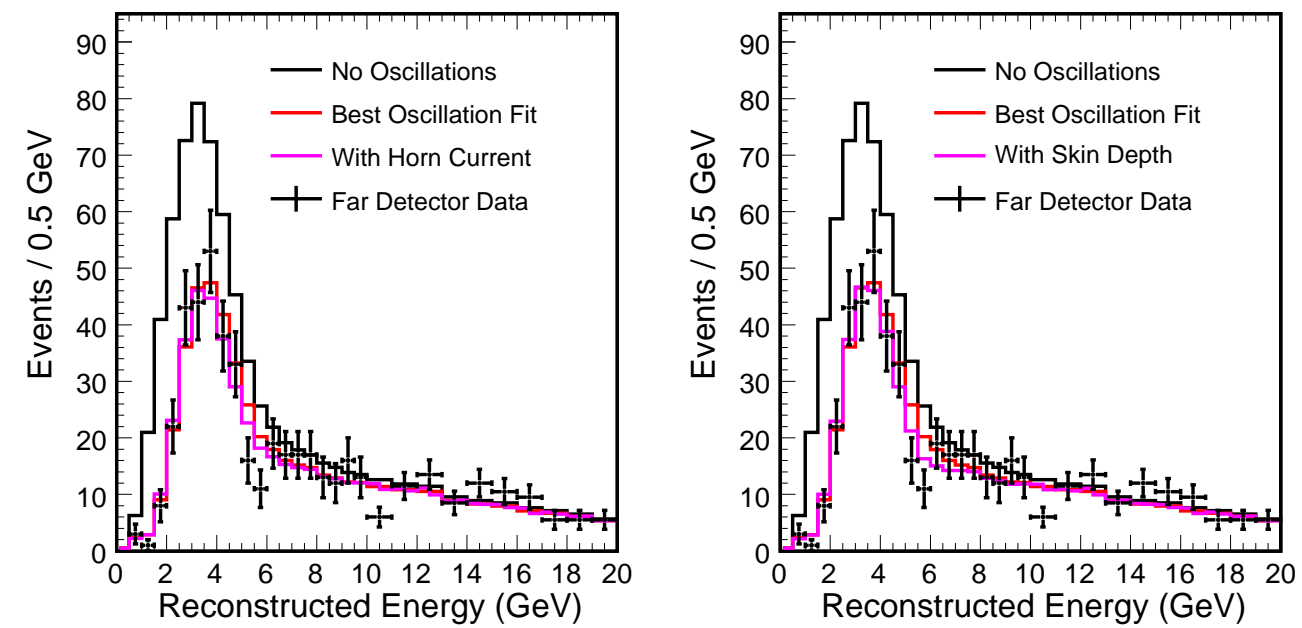

Figure 9.25: The effect of adding the horn current calibration (left) and the horn skin depth (right) as a parameter in the fit.

iii. The number of tracks with end $z$ position in the last few meters of both supermodules is lower than the expectation for events with neutrino energy between 3 and 7 $\mathrm{GeV}$.

A visual scan of all events between 4-7 GeV was done to see if the events being cut by the CC selection should not have been cut. One event had two short overlapping tracks and one other could possibly have been labeled as a CC event. Also, the events that are cut out all have $y$ values above 0.4 so these are not going to help explain the deficit. The scan also included examining the end of reconstructed tracks to see if missing hits were causing the observed deficit at the end of the supermodules. The scan showed 6 tracks missed one hit at the end of the track, 3 tracks were not really tracks, and only one track had 3 hits missing. The conclusion from these studies is that the deficit from 5-6 $\mathrm{GeV}$ is probably not due to an extrapolation or reconstruction problem, but a statistical fluctuation.

The collaboration approved looking at data from the blinded RunIII data set to see if the deficit still persisted. To prevent looking at data in the oscillation region, they authorized examining vents above $4 \mathrm{GeV}$. A comparison of the RunIII energy spectrum 
to the Beam Matrix extrapolation was performed in [93]. No deficit was seen for the RunIII data between 5 and $6 \mathrm{GeV}$, strengthening the assertion that the dip is likely a statistical fluctuation.

\subsection{Alternatives to Neutrino Oscillations}

Other processes can also produce a change in the observed $L / E$ dependence in neutrinos. The MINOS data can be used to test these other processes and compare it to the standard oscillation hypothesis.

\subsubsection{Neutrino Decay}

One possibility is that neutrinos do not oscillate, but decay. Neutrino decay implies that there is a mass difference between states, and therefore predicts mixing as well. Assuming two flavor mixing between $\nu_{\mu}$ and $\nu_{\tau}$ with eigenstates $\nu_{1}$ and $\nu_{2}$ The the muon neutrino survival probability is [94]:

$$
\begin{aligned}
P_{\nu_{\mu}->\nu_{\mu}}= & \left(\sin ^{4} \theta+\cos ^{4} \theta \exp (-\alpha L / E)\right)^{2} \\
& +2 \sin ^{2} \theta \cos ^{2} \theta \exp (-\alpha L / 2 E) \cos \left(\Delta m^{2} L / 2 E\right) .
\end{aligned}
$$

where $\alpha=m_{2} / \tau_{0}$ and $\tau_{0}$ is the rest-frame lifetime of the $\nu_{2}$ state. If $\Delta m_{32}^{2}$ is small the cosine term is unity and the equation reduces to:

$$
P_{\nu_{\mu}->\nu_{\mu}}=\left(\sin ^{2} \theta+\cos ^{2} \theta \exp (-\alpha L / 2 E)\right)^{2} .
$$

A fit was done using Equation 9.5, to the Far Detector Data. The best fit values were $\sin ^{2} \theta=0$, and $\alpha=2.45 \times 10^{-3}$ with a $\chi^{2}=112.3$ for the fit. The data favors oscillations 
over decay by $4.6 \sigma$. The ratio of the neutrino energy spectrum for the standard oscillation prediction and the decay prediction is shown in Figure 9.26

\subsubsection{Neutrino Decoherence}

It has long been postulated that quantum gravity may act as a background through which the neutrino propagates. If true, such a background would cause a change in the interference pattern. In [95], the formulation of quantum open systems was applied to the specific case of $\nu_{\mu} \rightarrow \nu_{\tau}$ transitions. The evolution of the probability of a $\nu_{\mu}$ remaining as a $\nu_{\mu}$ including the interaction with the background can be described by adding one additional parameter, $\mu^{2}$. The standard survival probability is modified in the following way:

$$
P_{\nu_{\mu}->\nu_{\mu}}=1-\frac{1}{2} \sin ^{2}(2 \theta)\left(1-\exp (-\kappa L / 2 E) \cos \left(\Delta m^{2} L / 2 E\right)\right)
$$

In the limit that $\kappa \rightarrow 0$, the standard oscillation formula is recovered. For the case of pure decoherence, $\Delta m_{32}^{2}=0$, the survival probability becomes:

$$
P_{\nu_{\mu}->\nu_{\mu}}=1-\frac{1}{2} \sin ^{2}(2 \theta) \cdot(1-\exp (-\kappa L / 2 E))
$$

The Far Detector data was fit to this case and the best fit values were found to be $\sin ^{2}\left(2 \theta_{32}\right)=1$ and $\kappa=0.011$. The $\chi^{2}$ value for the fit was 130.4 . For the case of pure decoherence the data favors oscillations by $6.3 \sigma$. Figure 9.26 compares the oscillation hypothesis to neutrino decay.

\subsubsection{Lorentz and CPT Invariance Violation}

The introduction of of CPT Invariance or Lorentz Invariance Violation can cause different neutrino mass states to propagate with slightly different velocities. This can lead to an unconventional energy dependence of the oscillations. The MINOS data can be fit to see 


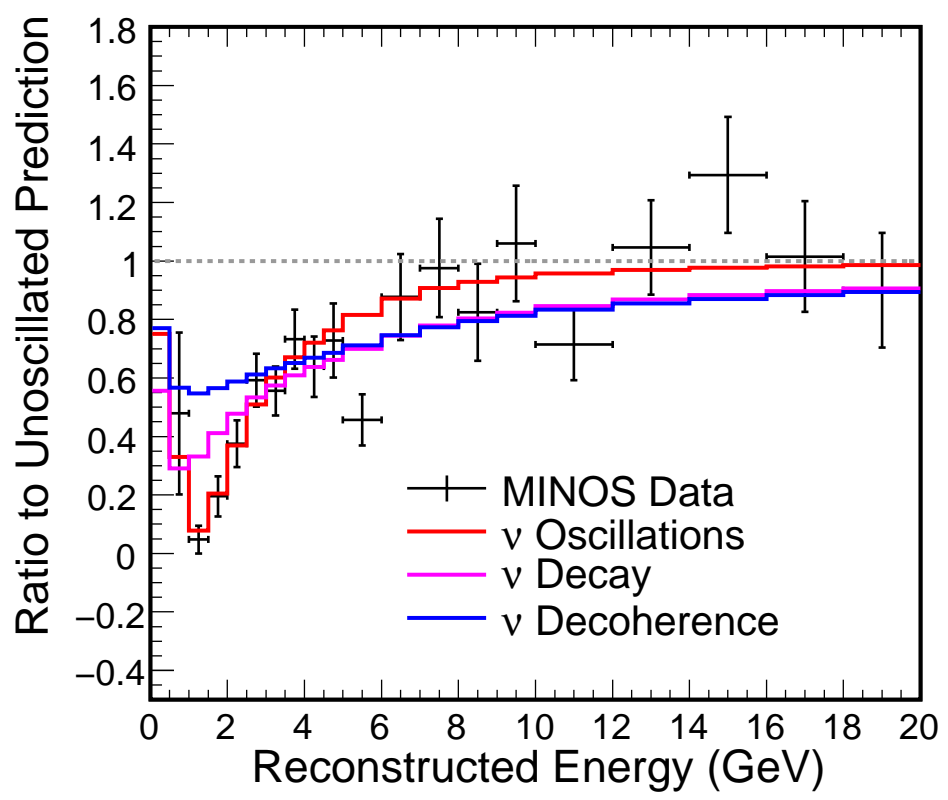

Figure 9.26: The ratio of the unoscillated spectrum to the best fit values for different models: data (black points), oscillations(red), decay (red), decoherence (blue).

the sensitivity to the energy exponent:

$$
P\left(\nu_{\mu}->\nu_{\mu}\right)=1-\sin ^{2} 2(\theta) \sin \left(1.27 \Delta m_{32}^{2} L / E^{n}\right),
$$

where $n=1$ for standard oscillations. Figure 9.27 shows the $\chi^{2}$ function as a function of $n$. Each point in the figure is minimized with respect to $\Delta m_{32}^{2}$ and $\sin ^{2}\left(2 \theta_{32}\right)$. The best fit value is $n=0.98 \pm 0.12$, but there is also a local minimum closer to zero at $n=0.11$. The oscillation parameters at this point are $\sin ^{2}\left(2 \theta_{32}\right)=0.95$, and $\Delta m_{32}^{2}=4.8 \times 10^{-3} \mathrm{eV}^{2}$. The difference in $\chi^{2}$ values between the absolute minimum and this local minimum at $n=0.11$ is $\Delta \chi^{2}=11$. The ratio to the unoscillated spectrum is shown in Figure 9.28.

Some specific models have been suggested that apply admixtures of CPT and oscillations. These predict the the following consequences for the standard survival probability [96, 97]:

$$
P_{\nu_{\mu}->\nu_{\mu}}=\sin ^{2}\left(2 \theta_{32}\right) \sin ^{2}\left(1.27 \Delta m_{32}^{2} \frac{L}{E} \pm \alpha L\right)
$$




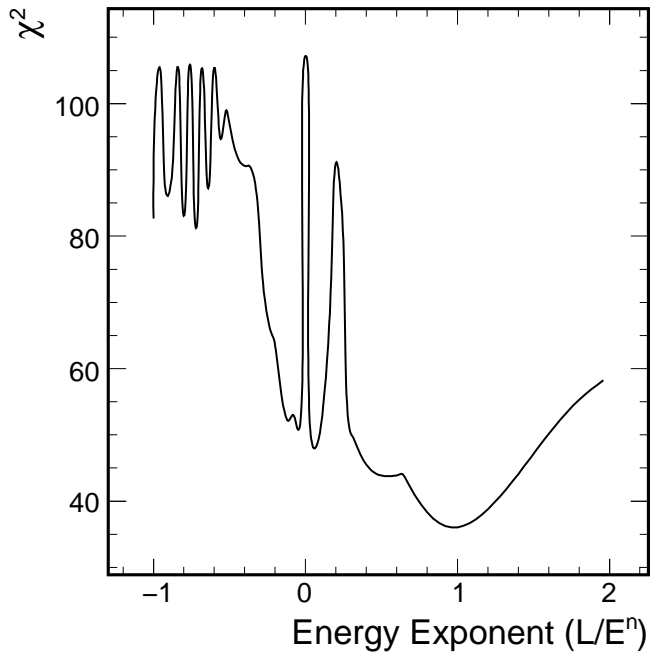

Figure 9.27: The $\chi^{2}$ values as a function of the exponent of the energy in the denominator. Each point is minimized with respect to the oscillation parameters.

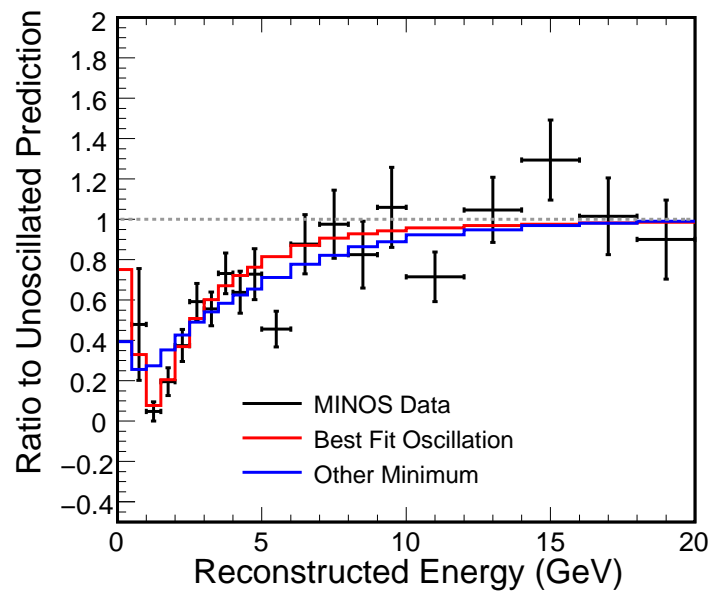

Figure 9.28: A ratio of the predicted spectrum to the unoscillated prediction for: the data (black points), the standard oscillation scenario (red) and the prediction from the local minimum of $n=0.11, \sin ^{2}\left(2 \theta_{32}\right)=0.95$ and $\Delta m_{32}^{2}=4.8 \times 10^{-3} \mathrm{eV}^{2}$. 


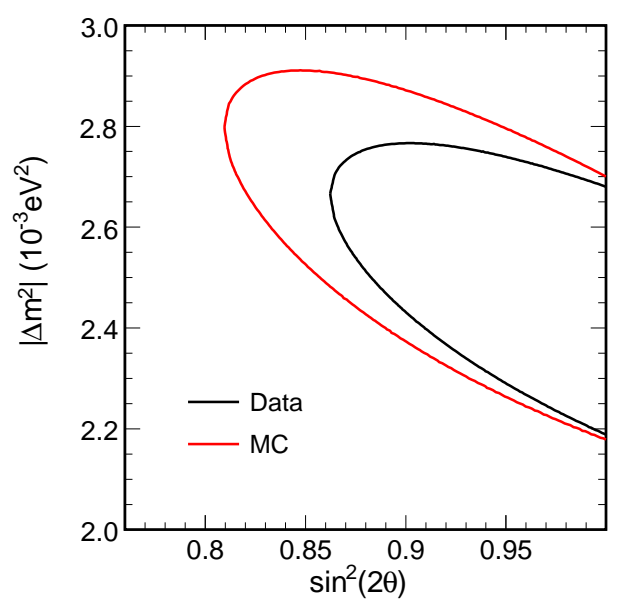

Figure 9.29: The 90\% C.L. on the oscillation parameters of the data (black) and Monte Carlo (red) for $3.36 \times 10^{20}$ POT.

and,

$$
P_{\nu_{\mu}->\nu_{\mu}}=\sin ^{2}\left(2 \theta_{32}\right) \sin ^{2}\left(1.27 \Delta m_{32}^{2} \frac{L}{E} \pm \beta L\right)
$$

where the "+" sign is for neutrinos and "-" for antineutrinos. The MINOS data is able to set limits on $\alpha$ and $\beta$. The $90 \%$ C.L. limits are:

$$
\begin{aligned}
& \alpha<2 \times 10^{-4} \mathrm{~km}^{-1} \\
& \beta<6 \times 10^{-6} \mathrm{GeV}^{-1} \mathrm{~km}^{-1} .
\end{aligned}
$$

\subsection{Measurements at a Boundary}

The $90 \%$ C.L. is compared to the sensitivty derived from Monte Carlo for the same exposure in Figure 9.29. The results from the data are better than would be expected from the Monte Carlo, especially for the mixing angle. Two potential causes for this, are that the contour is pulled by the best fit point being in the unphysical region and statistical fluctuations in the data. 
The procedure for finding the confidence intervals can also be affected by the presence of a physical boundary. A method developed to find confidence intervals that fully takes into account the presence of a physical boundary was developed by Feldman and Cousins [98]. The application of the method requires a large number of pseudoexperiments at each point in the parameter space. The confidence limits can be determined by these large number of pseudo-experiments in the following way:

i. On a grid of $\Delta m_{32}^{2}$ and $\sin ^{2}\left(2 \theta_{32}\right)$ (restricted to the physical region), 10,000 fake experiments (oscillated by the parameters at the grid point) are generated at each grid point by Poisson fluctuating the number of events in each bin from the predicted Monte Carlo energy spectrum at $3.2 \times 10^{20}$ POT.

ii. Perform an oscillation fit to each fake experiment. Define $\Delta \chi^{2}$ as the difference between the $\chi^{2}$ from the best fit point and the $\chi^{2}$ from a prediction using the oscillation parameters of the grid point.

iii. Each grid point will give 10,000 values of $\Delta \chi^{2}$. The value which encompasses $90 \%$ of these experiments is the $\Delta \chi_{90}^{2}$ point, or the point which should be used to find the $90 \%$ confidence region.

The grid size was chosen to encompass the region of parameter space where MINOS has sensitivity: $0.7-1.0$ for $\sin ^{2}\left(2 \theta_{32}\right)$ and $1.5-3.5 \times 10^{-3} \mathrm{eV}^{2}$ for $\Delta m_{32}^{2}$. Figure 9.30 shows the $\Delta \chi_{90}^{2}$ grid values for an exposure of $3.2 \times 10^{20}$ POT. The Gaussian approximation uses the value of 4.61 to define the $90 \%$ confidence region. As expected, values far from the boundary are close to 4.61 , but close to $\sin ^{2}\left(2 \theta_{32}\right)=1$ the $\Delta \chi_{90}^{2}$ values drop below 4 . The reason the values drop is that a number of values will pile up for $\sin ^{2}\left(2 \theta_{32}\right)=1$, because they want to go into the unphysical region. This will lower the $\Delta \chi_{90}^{2}$ surface. Figure 9.30 illustrates this effect by plotting the $\Delta \chi_{90}^{2}$ for two separate points, one far away and one close to the boundary.

The corrected $90 \%$ C.L. can now be found by using $\Delta \chi^{2}=\chi_{\min }^{2}+\Delta \chi_{90}^{2}$. Figure 9.31 compares this contour with the one calculated by $\Delta \chi^{2}=\chi_{\min }^{2}+4.61$. This plot shows 

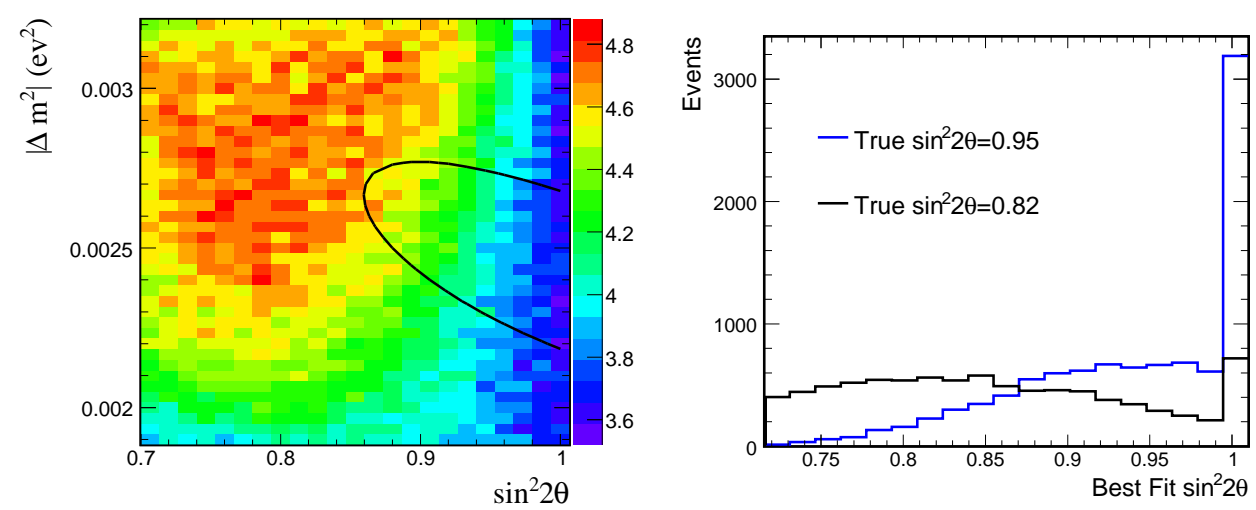

Figure 9.30: Left: The $\Delta \chi_{90}^{2}$ surface is indicated by the color. The black line shows the $90 \%$ C.L. from the MINOS data. Right: $\Delta \chi^{2}$ values for two different points: $\sin ^{2}\left(2 \theta_{32}\right)$ $=0.82$ and 0.95 .

that when the effect of the physical boundary on the oscillation analysis is to broaden the limits on $\Delta m_{32}^{2}$ near the boundary of $\sin ^{2}\left(2 \theta_{32}\right)=1$.

\section{$9.9 \quad$ Future Sensitivity}

At what point in the future do the systematic uncertainties begin to dominate over the statistical errors? The method employed to ascertain this point uses generated Monte Carlo at a number of possible exposures from 5 to $200 \times 10^{20}$ POT. It proceeds as follows:

i. Oscillate the Monte Carlo with the oscillation parameters close to the minimum $\left(\Delta m_{32}^{2}=2.43 \times 10^{-3} \mathrm{eV}^{2}, \sin ^{2}\left(2 \theta_{32}\right)=1.0\right)$ to generate fake data samples and scale it to the desired exposure.

ii. Generate 10,000 fake experiments by Poisson fluctuating the total number of events and randomly drawing that number of events from the Monte Carlo.

iii. For the most important systematic errors, randomly select values from a Gaussian with the width equal to the uncertainty. The systematic errors used are the normalization (4\% uncertainty), shower energy scale (3.3\% uncertainty), and the neutral current background (50\% uncertainty). 


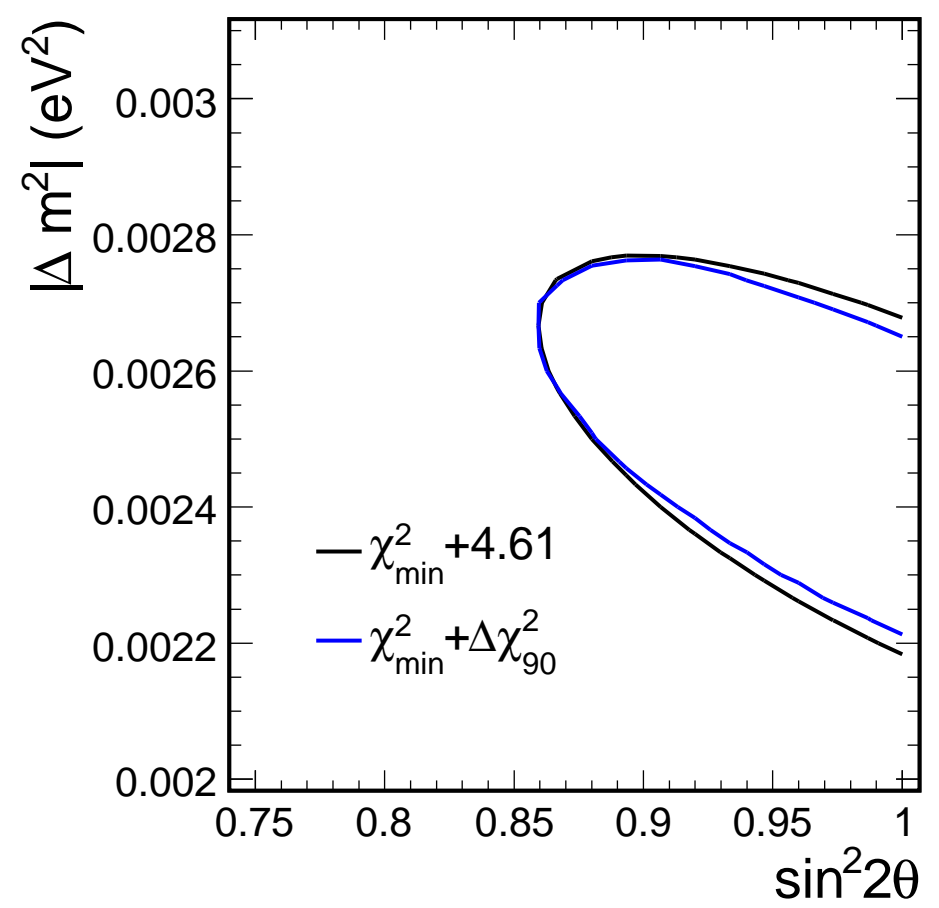

Figure 9.31: The 90\% C.L. of the LE data found by using a Feldman-Cousins prescription (blue) and the Gaussian approximation (black). 
iv. Fit the Monte Carlo to the randomly selected fake data and evaluate the best fit oscillation and systematic parameters comparing them to the actual values used.

Figure 9.32 shows the difference between the best fit and true values of the oscillation parameters for exposures of 10 and $40 \times 10^{20}$ POT. Several variations of the above were carried out:

i. Excluding systematic errors

ii. Including systematic errors, but not explicitly fitting for them

iii. Including systematic errors, and fitting for them

iv. Including systematic errors, but removing the Poisson fluctuations.

Each of these variations were performed at each exposure point. Figure 9.33 is a comparison of the different methods and the errors of the oscillation parameters as a function of exposure. The errors are evaluated by taking the width of the Gaussian fit to the difference between the best fit and true values. To see possible deviations from $\sqrt{N}$ scaling, the errors are also plotted as a function of $1-1 / \sqrt{\text { exposure }}$ see (Figure 9.34). Errors that scale as $\sqrt{N}$ on such an axis will be straight lines.

The main conclusion from these plots is that the oscillation parameters do not appear to hit a systematic floor. As the data increases, if the oscillation parameters are included in the fit, they are able to be better constrained. If the systematic errors are not included in the fit (the red curve in Figure 9.34) then the error does begin to deviate from $\sqrt{N}$ scaling at about $20 \times 10^{20}$ POT.

Figure 9.35 shows the error on the systematic parameters as a function of exposure. The error is calculated the same as before. It shows that the normalization parameter is constrained the best (i.e. has the smallest error). This is true because the high energy tail of the spectrum sets the normalization scale. The NC background, because of the small contamination has the the largest of the three. The systematic parameters appear to hit a threshold, after which the error is significantly reduced. This happens at about $100 \times 10^{20}$ POT for the NC background and $50 \times 10^{20}$ POT for the shower energy. This 

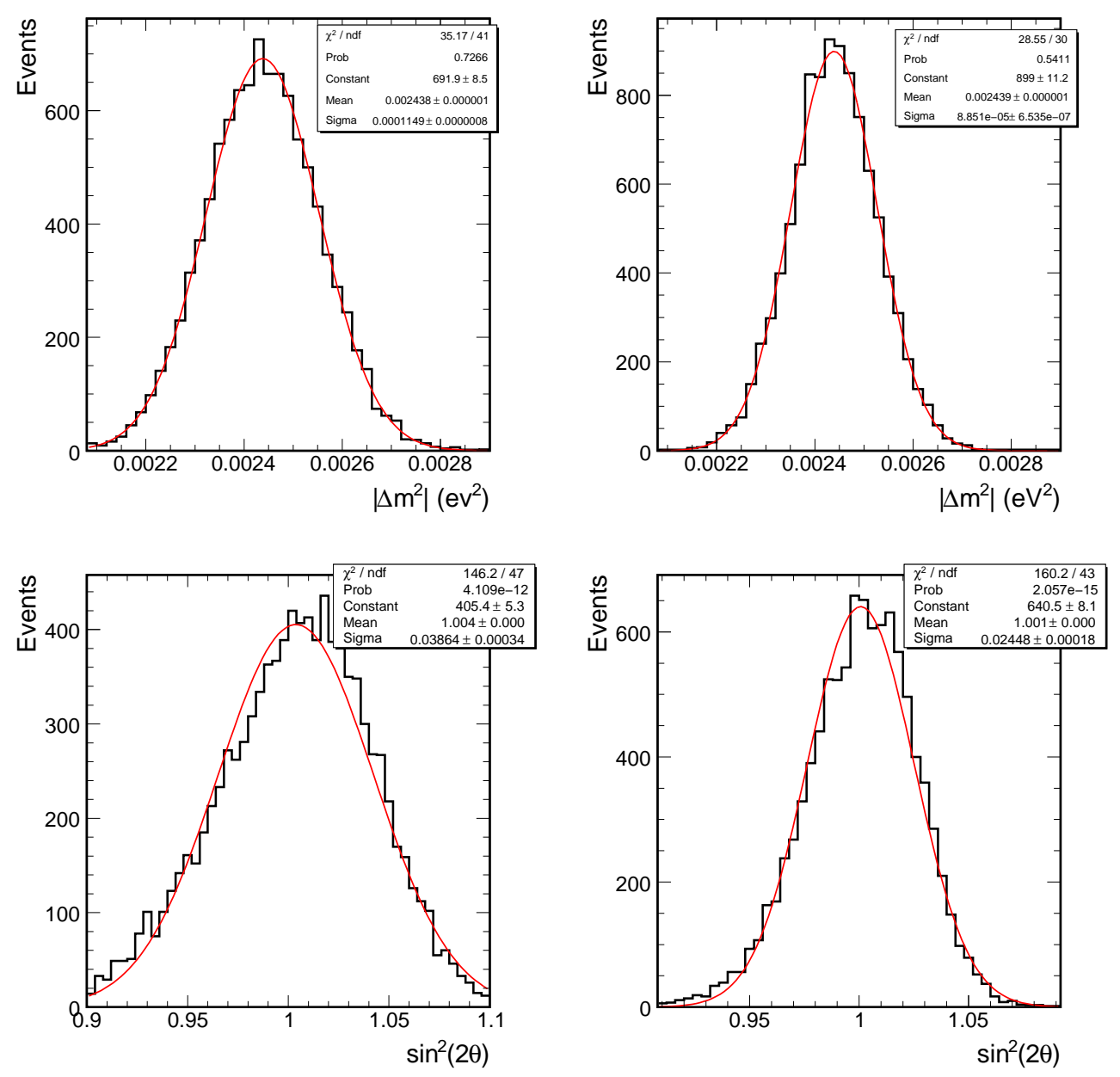

Figure 9.32: The distribution of oscillation parameters for 10,000 different fake experiments at exposures of $10 \times 10^{20}$ POT (left) and $40 \times 10^{20}$ POT (right). The width of a Gaussian fit to the distribution is used as the error. 

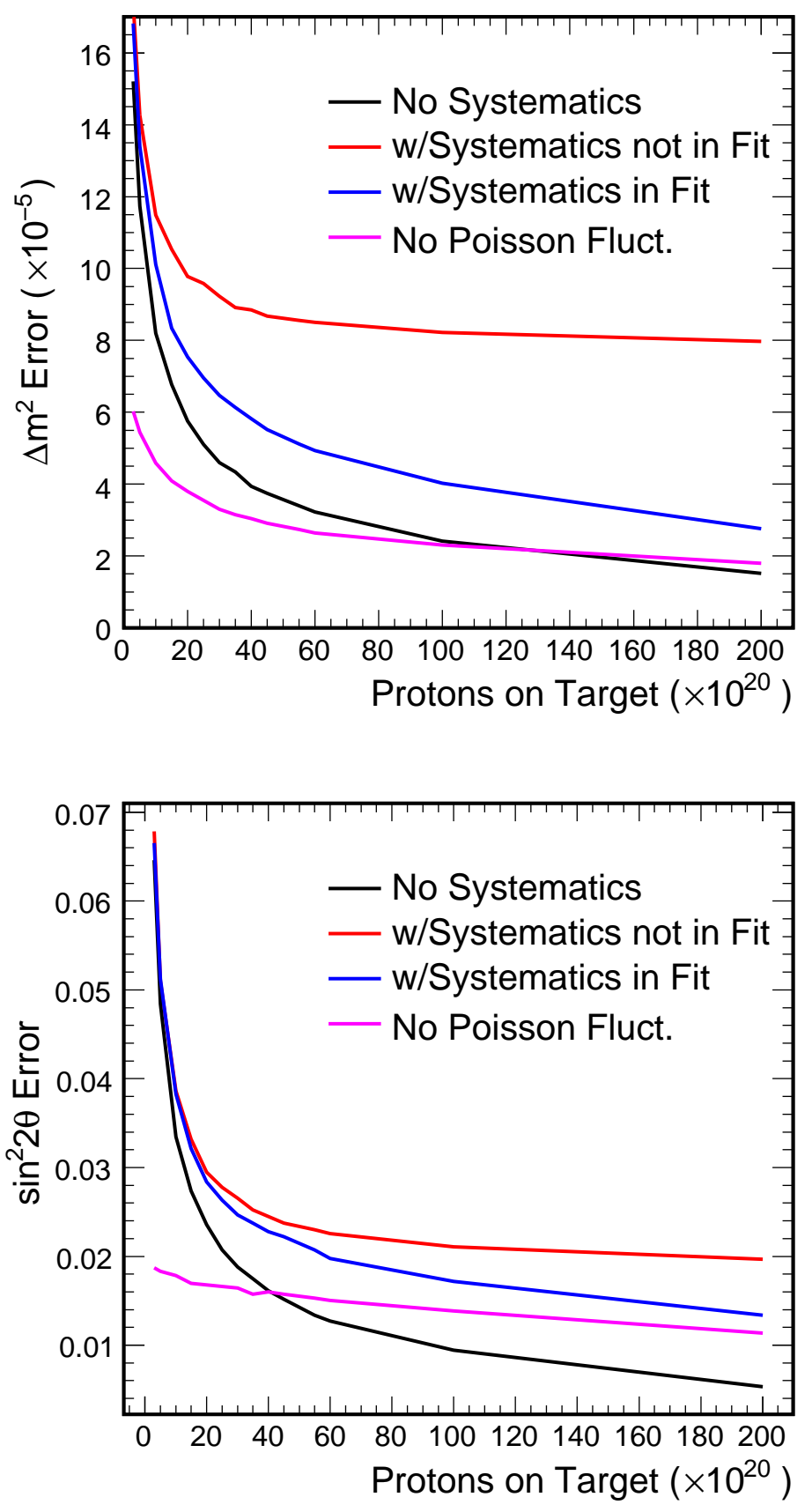

Figure 9.33: The error on the oscillation parameters $\Delta m_{32}^{2}$ (top) and $\sin ^{2}\left(2 \theta_{32}\right)$ (bottom) as a function of POT. Each point represents the width of a Gaussian fit to 10,000 fake experiments for experiments with: no systematics (black), including systematics but not including them in the fit (red), including systematics in the fit (blue), including systematics but without statistical fluctuations (purple). 

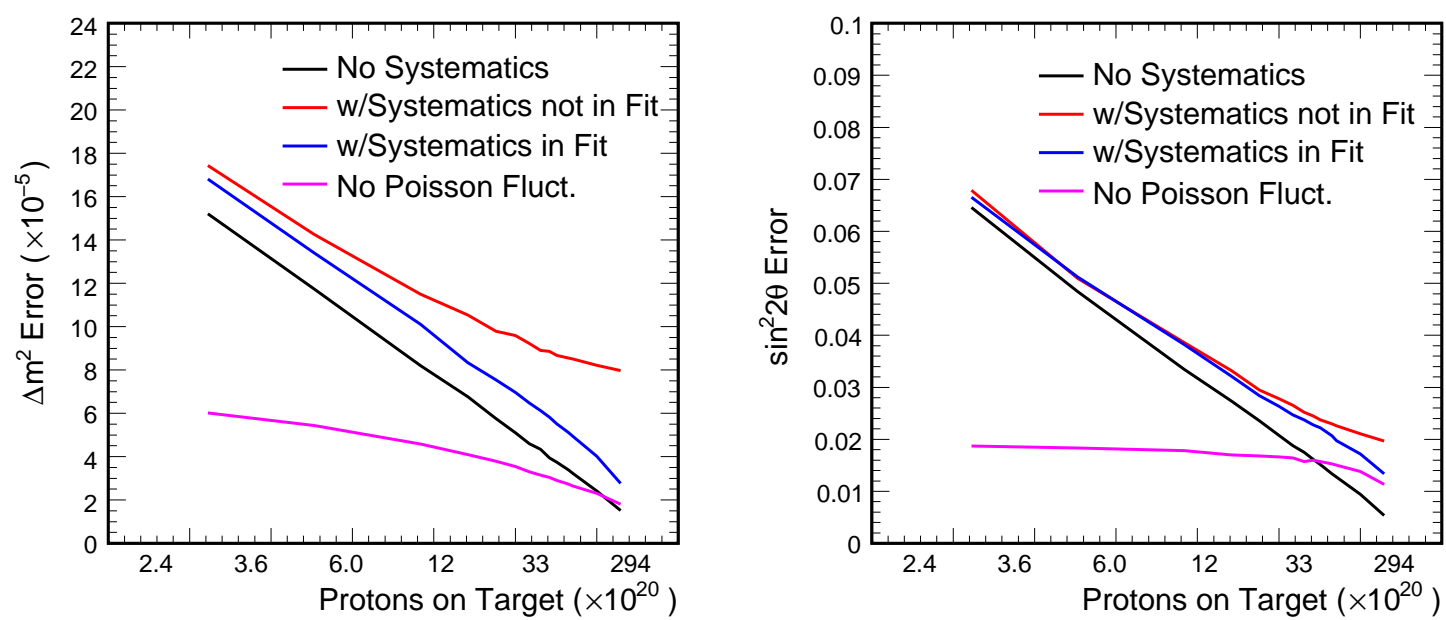

Figure 9.34: The error on the oscillation parameters $\Delta m_{32}^{2}$ (left) and $\sin ^{2}\left(2 \theta_{32}\right)$ (right) as

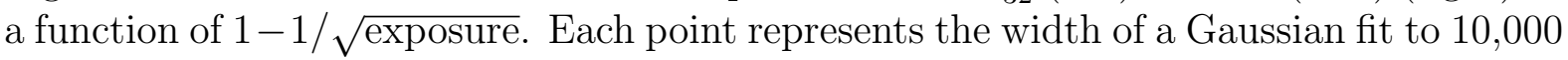
fake experiments for experiments with: no systematics (black), including systematics but not including them in the fit (red), including systematics in the fit (blue), including systematics but without statistical fluctuations (purple).

may be the point where the statistical errors are reduced enough to have sensitivity to the parameters.

To see where the systematic errors become the same size as the statistical errors one can look to see in Figure 9.34 where the purple curve (systematics only) intersects the black (statistics only). They become comparable at $\sim 40 \times 10^{20}$ POT. 

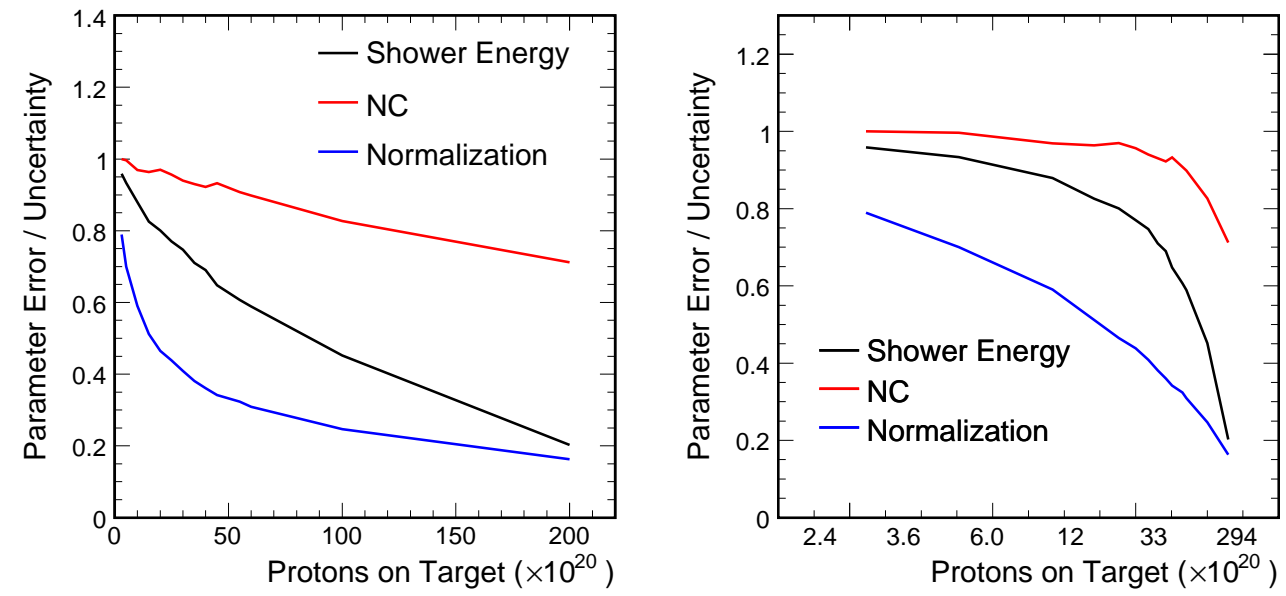

Figure 9.35: The error on the systematic parameters: the neutral current background (red), the relative shower energy (black) and the relative normalization (black) as function of exposure (left) and as function of $1-1 / \sqrt{\text { exposure }}$ (right). Each point represents the width of a Gaussian fit to 10,000 fake experiments. 


\section{Chapter 10}

\section{Summary and Outlook}

This thesis has described a precision measurement of the neutrino oscillation parameters first observed in atmospheric neutrino experiments. The number of $\nu_{\mu}$ neutrinos was measured in both the MINOS Near and Far Detectors. The data used in this analysis was taken from May 2005 to June 2007 for a total of $3.36 \times 10^{20}$ protons on target. To compare the data observed at the Far Detector a prediction of the expectations without neutrino oscillations was necessary.

A method of extrapolation was developed that used the data in the Near Detector to correct for our lack of knowledge of the neutrino production. The Monte Carlo was fit to data collected from 10 different running conditions in the Near Detector. The Monte Carlo was fit to these different data sets to provide an improved prediction. In addition to providing a method of extrapolation the fit reduced the uncertainties from hadron production. The prediction from the improved Monte Carlo was thoroughly studied in the Near Detector before being used to extrapolate the flux to the Far Detector.

Different methods for distinguishing between $\mathrm{CC}$ and $\mathrm{NC}$ were studied. The kNN method was seen to have the best sensitivity to oscillations. The largest systematic uncertainties were identified by applying shifts in fake data samples and doing an oscillation analysis. The most important systematic uncertainties were: the relative normalization between Near and Far, the hadronic energy scale and the residual difference in the Near 
Detector from the fit. These were included as nuisance parameters in the analysis of the Far Detector data..

A total of 848 events were observed in three beam configurations in the Far Detector while 1064 were expected with oscillations. A clear energy dependent deficit was seen in the number of $\nu_{\mu}$ events. The data was fit assuming $\nu_{\mu} \rightarrow \nu_{\tau}$ oscillations. The results of the fit are $\Delta m_{32}^{2}=2.45_{+0.12}^{-0.12} \times 10^{-3} \mathrm{eV}^{2}$ and $\sin ^{2}\left(2 \theta_{32}\right)=1_{-0.04}$ and $\sin ^{2}\left(2 \theta_{32}\right) \leq 0.90(90 \%$ C.L.) when the mixing angle is constrained to the physical region. When the constraint on $\sin ^{2}\left(2 \theta_{32}\right)$ is relaxed the best fit values become $\Delta m_{32}^{2}=2.32_{+0.18}^{-0.15} \times 10^{-3} \mathrm{eV}^{2}$ and $\sin ^{2}\left(2 \theta_{32}\right)$ $=1.09_{-0.09}^{+0.07}$ and $\sin ^{2}\left(2 \theta_{32}\right) \leq 0.90$ (90\% C.L.) Figure 10.1 compares the limits from this analysis compared to other experiments. MINOS gives the best constraints on $\Delta m_{32}^{2}$.

At the current time MINOS has accumulated over $7.5 \times 10^{20}$ POT, more than double the amount of data presented in this thesis. The future sensitivity to the oscillation parameters will continue to improve as shown in Figure 10.2. 


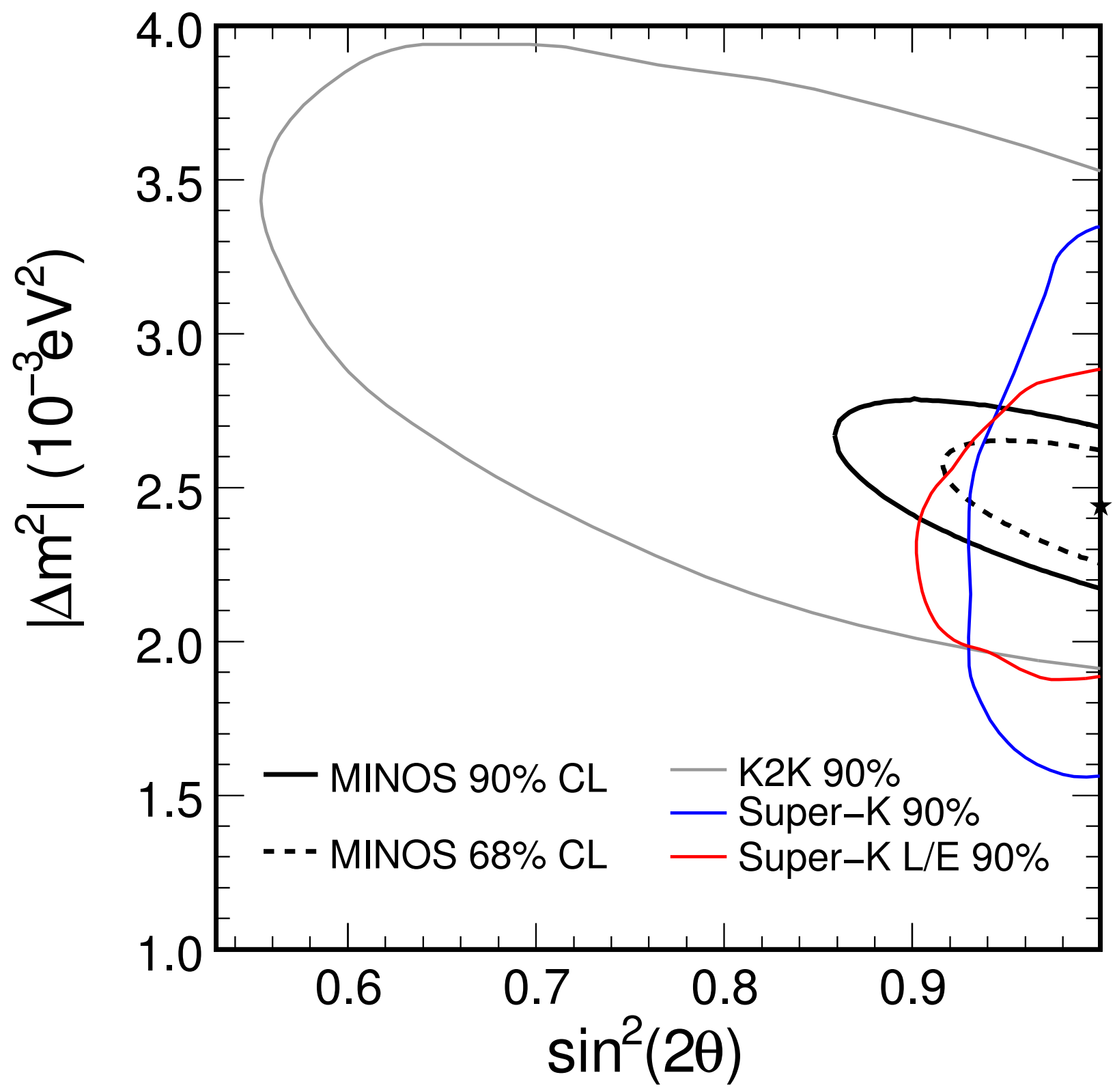

Figure 10.1: The $68 \%$ and $90 \%$ C.L. of this analysis compared with other measurements of the oscillation parameters from SuperKamiokande (blue), the $L / E$ analysis of SuperKamiokande (red) and K2K (gray). systematic errors. 


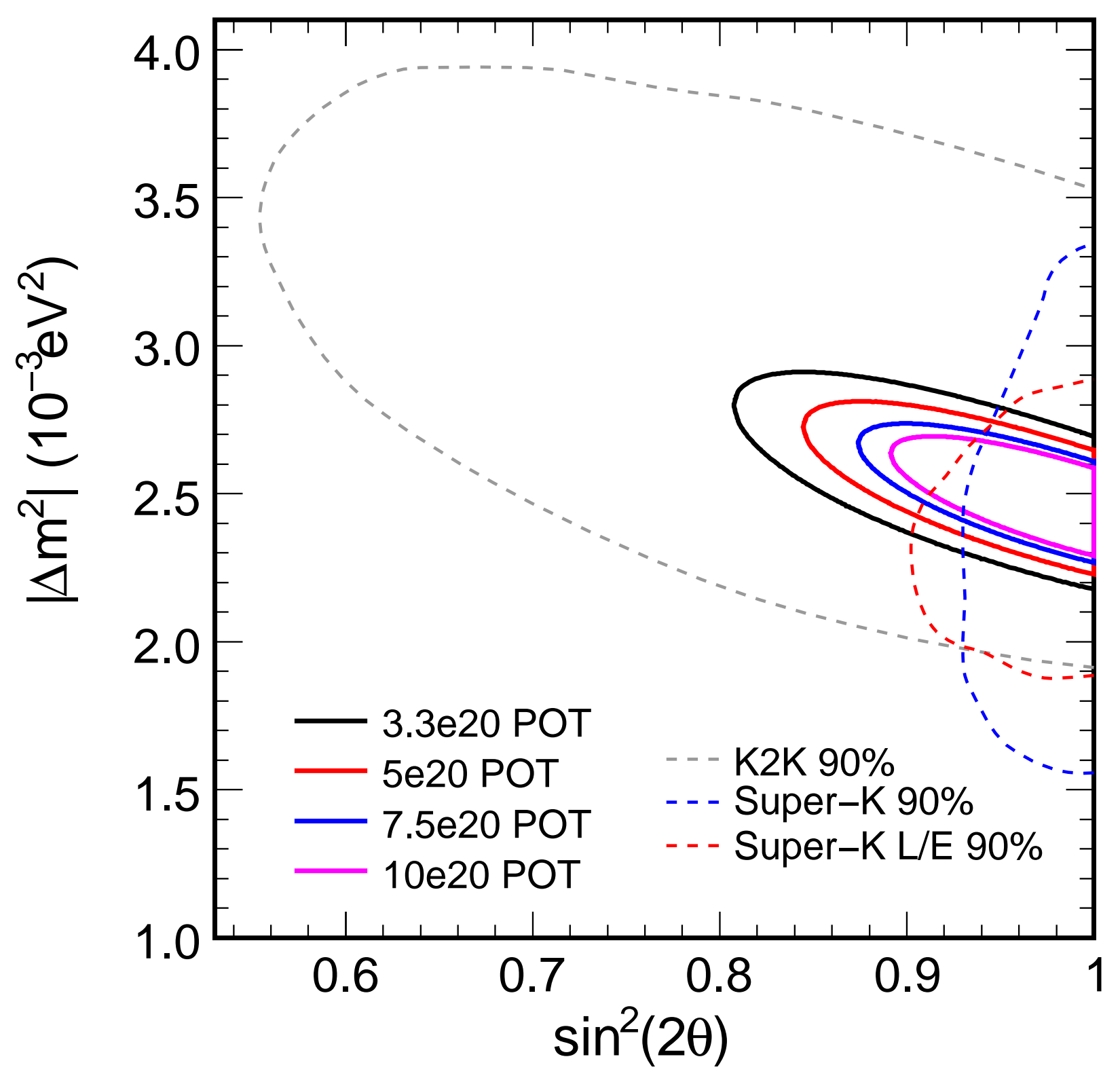

Figure 10.2: The sensitivity with increasing POT. 


\section{Appendix A}

\section{Uncertainties to the $\nu_{e}$ Flux}

The impact of different elements of the focusing system and alignment on the flux has only been thoroughly studied for the $\nu_{\mu}$ flux. To accommodate the need to calculate the impact of a large number of systematic effects, the PBEAM [99] Monte Carlo was used instead of GNUMI.

The speed, however, comes at a cost in that the calculation does not handle cascade decays such as $\pi \rightarrow \mu \rightarrow \nu_{e}$, which are an important component of the background $\nu_{e}$ flux. For the $\nu_{e}$ appearance measurement it will be important to know the systematic error on the number of $\nu_{e}$ background event due to beam alignment and focusing, but PBEAM cannot be used to perform such a calculation.

The problem with using GNUMI to simulate systematic effects for the $\nu_{e}$ flux is that of computing. The GNUMI simulation program takes considerably more time than PBEAM, but is able to track the $\nu_{e}$ component of the flux. A further complication is the inherently low rate of $\nu_{e}$ events. Only $1.3 \%$ of the NuMI beam comes from electron neutrinos, therefore a large sample of Monte Carlo is needed. With such low statistics, only an estimate of the uncertainty on the total number of $\nu_{e}$ events. As requested by the $\nu_{e}$ Analysis group, the uncertainty will be calculated for the total number of $\nu_{e}$ events with true energy less than $10 \mathrm{GeV}$. This study will be restricted to the beam configurations 
relevant to the $\nu_{e}$ appearance measurement which are the le010z185 (LE), and le250z200i (HE) configurations.

The most important beam related uncertainties from [47] are:

i. Horn 1 Offset (Estimated uncertainty: $1 \mathrm{~mm}$ ) - This is a shift in the horizontal or vertical position (transverse to the beam line) of Horn 1. A beam based measurement of the vertical and horizontal position of Horn 1 was done using the primary proton beam [100]. The beam was scanned vertically and horizontally while the target was removed and observations were done of where the beam scattered off the alignment bars. This study found the position of horn 1 to be off by $0.8 \pm 0.3 \mathrm{~mm}$ in the $x$, or horizontal, direction and $0.6 \pm 0.8 \mathrm{~mm}$ in the $y$, or vertical direction.

ii. Horn Current Miscalibration (Estimated uncertainty: 1\%) - The uncertainty in the absolute current through the horns was determined to be stable to less than $1 \%$ [101]. It was also observed that the actual current was $1.6 \%$ less than the desired value. The Monte Carlo used in the simulation was generated with this reduced current.

iii. Horn Current Distribution (Estimated uncertainty: $\delta=6 \mathrm{~mm} / \delta=\infty$ ) - For an ideal conductor all the current should run on the outer surface of the horn, but for a realistic case the current will penetrate into the bulk of the conductor some depth $\delta$ given by the skin depth. This can modify the magnetic field in the horns and correspondingly the focusing of hadrons. In the Monte Carlo $\delta=\infty$, which uniformly distributes the current throughout the conductor.

iv. Target $z$ (Estimated uncertainty : $1 \mathrm{~cm}$ ) - The distance between the target and the focusing horns determines the energy of the neutrino beam.

More detailed discussions of each of these effects is found in [47]. 


\section{A.1 Analysis Procedure}

For each effect, large Monte Carlo samples were generated corresponding to $\pm 1 \sigma, \pm 2 \sigma$, and $\pm 3 \sigma$ shifts. A Monte Carlo sample of 1 billion protons on target was generated for the raw unshifted Monte Carlo to remove statistical fluctuations. This is five times the size of the available GNUMI Monte Carlo. As mentioned previously (see Section 4.3), GNUMI does not simulate the initial proton interaction, but rather uses FLUKA to interact the protons in the NUMI target. Only 200 million protons on the NuMI target generated using FLUKA were available. To achieve the high number of events required the FLUKA files were reused with different random seeds. The random seed determines the decay points of the hadrons and the kinematics.

Figure A.1 shows the ratio of each of the different effects relative to the nominal flux for the LE configuration and Figure A.2 for the HE configuration as a function of energy. The cross-sections have been folded into these plots to directly see the change in the number of $\nu_{e}$ events.

To find the total change in the number of $\nu_{e}$ events due to each effect the total events less than $10 \mathrm{GeV}$ are summed. Figures A.3 and A.4 show the ratio of this sum to the nominal flux. These points are fit to a linear function and the error is evaluated by the value of this fitted function at $1 \sigma$. All errors except the horn 1 offset are symmetric about zero and the error is averaged by

$$
\text { error }=(f(+1 \sigma)-f(-1 \sigma)) / 2,
$$

where $f$ is the fitted function. For the horn 1 offset the value at $1 \sigma, f(+1 \sigma))$, is used. This number is the estimate on the systematic uncertainty on the $\nu_{e}$ events less than 10 $\mathrm{GeV}$. The uncertainty on this number is found by evaluating the errors from the fit. 

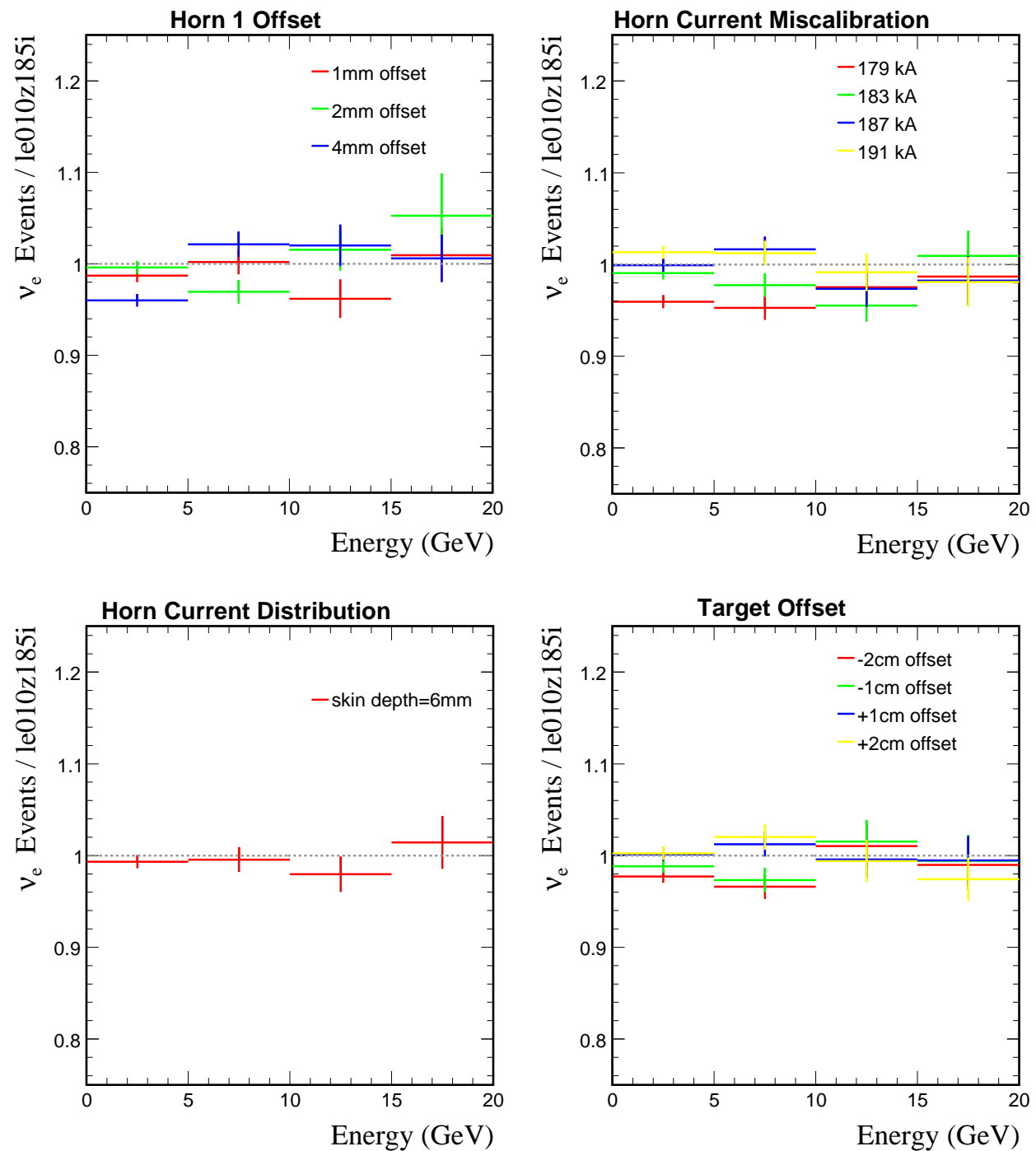

Figure A.1: The relative change on the number of $\nu_{e}$ events for different effects: horn 1 offset (upper left), horn current miscalibration (upper right), horn current distribution (lower left), and target $z$ (lower right). These changes are relative to the LE beam. 

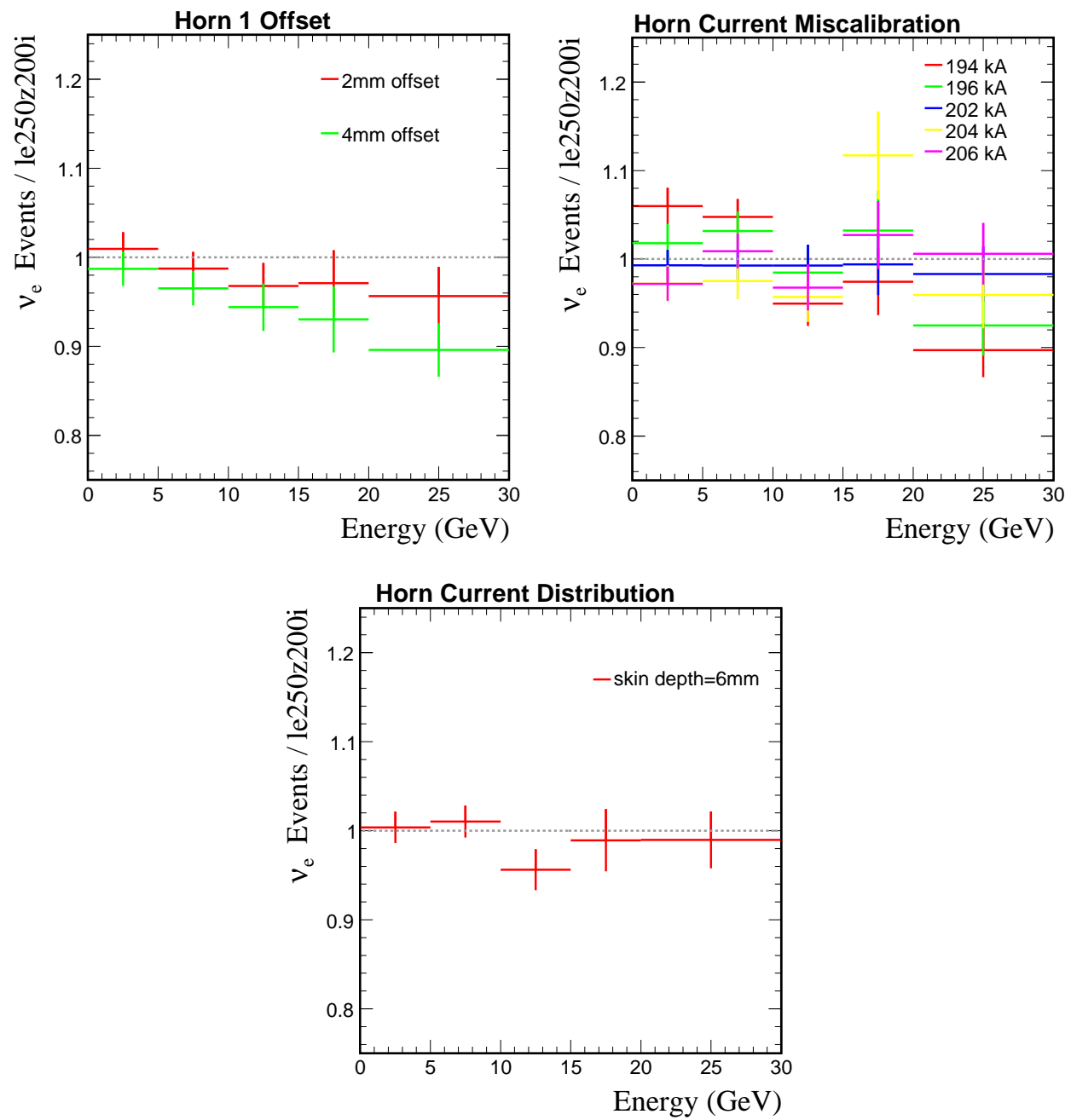

Figure A.2: The relative change on the number of $\nu_{e}$ events for different effects: horn 1 offset (upper left), horn current miscalibration (upper right), horn current distribution (lower left), and target $z$ (lower right). These changes are relative to the HE beam. 

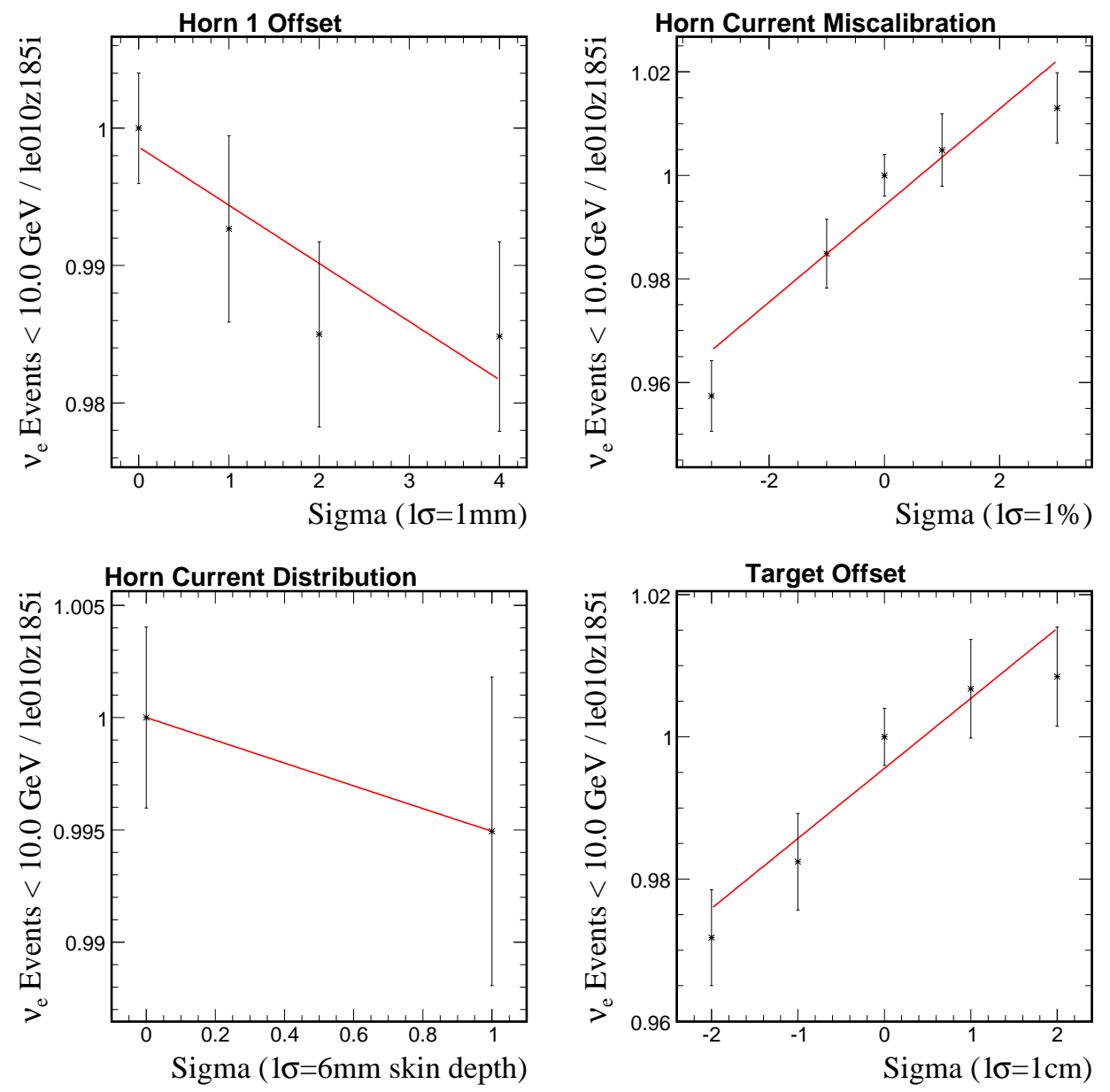

Figure A.3: The number of $\nu_{e}$ events for the LE beam less than $10 \mathrm{GeV}$ for each effect divided by that of the nominal Monte Carlo. It is plotted as a function of $\sigma$ away from the nominal Monte Carlo. 

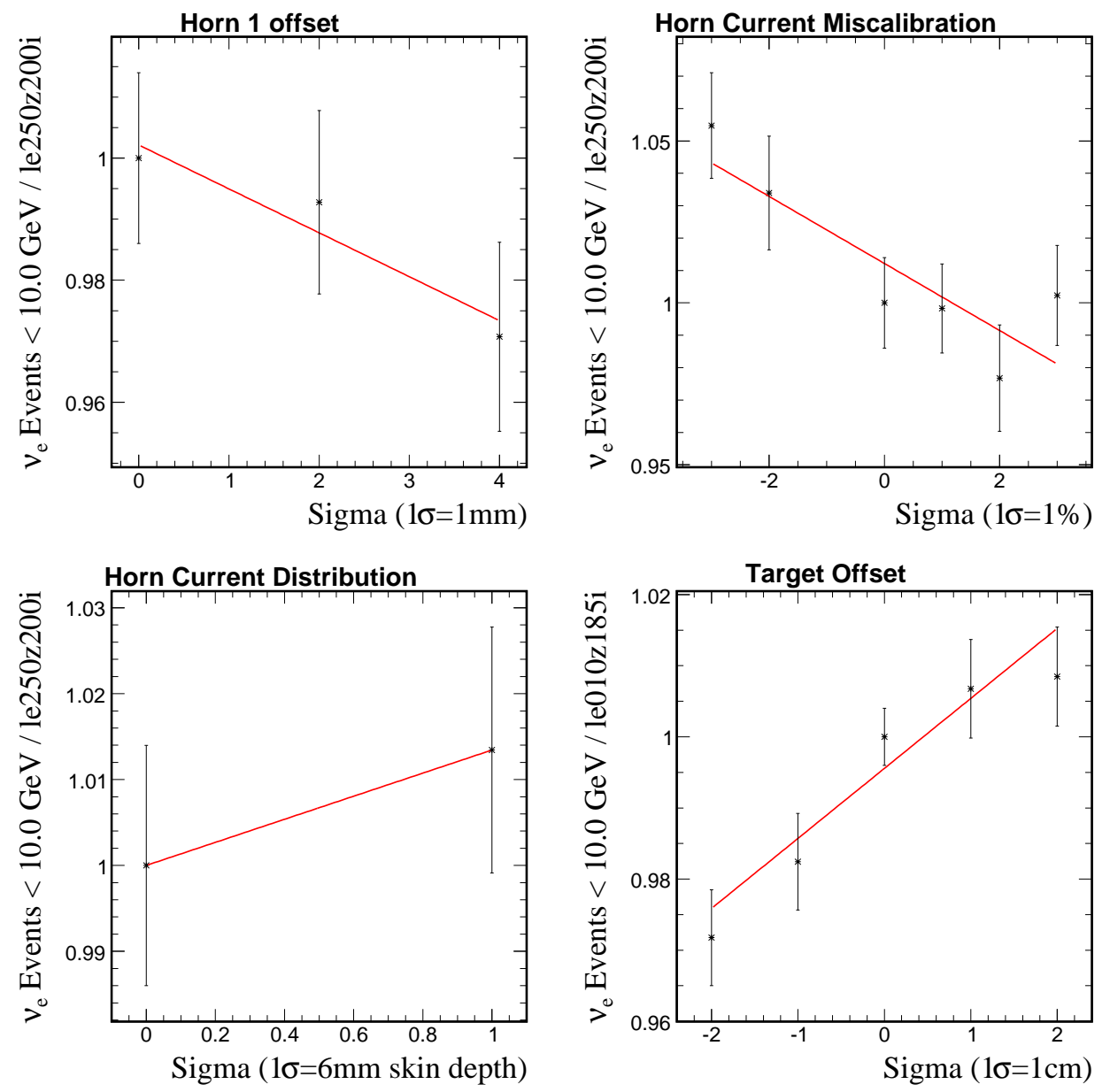

Figure A.4: The number of $\nu_{e}$ events for the HE beam less than $10 \mathrm{GeV}$ for each effect divided by that of the nominal Monte Carlo. It is plotted as a function of $\sigma$ away from the nominal Monte Carlo. 


\begin{tabular}{lc}
\hline \hline Effect & Change in $\nu_{e}$ Events $<10 \mathrm{GeV}$ \\
\hline Horn 1 Offset & $0.56 \pm 0.19 \%$ \\
Horn Current Miscalibration & $0.93 \pm 0.15 \%$ \\
Horn Current Distribution & $0.51 \pm 0.8 \%$ \\
Target $z$ & $0.98 \pm 0.22 \%$ \\
POT & $2 \%$ \\
Total & $2.67 \%$ \\
\hline \hline
\end{tabular}

Table A.1: The systematic errors on the number of $\nu_{e}$ events with less than $10 \mathrm{GeV}$ for the LE beam configuration.

\begin{tabular}{lc}
\hline \hline Effect & Change in $\nu_{e}$ Events $<10 \mathrm{GeV}$ \\
\hline Horn 1 Offset & $0.50 \pm 0.52 \%$ \\
Horn Current Miscalibration & $1.04 \pm 0.31 \%$ \\
Horn Current Distribution & $1.34 \pm 2.00 \%$ \\
POT & $2 \%$ \\
Total & $2.8 \%$ \\
\hline \hline
\end{tabular}

Table A.2: The systematic error on the number of $\nu_{e}$ events with less than $10 \mathrm{GeV}$ for the HE beam configuration.

\section{A.2 Results}

Tables A.1 and A.2 summarize the results for each of the systematic effects considered. An additional $2 \%$ error on the number of protons on target (POT) has also been added for each of the beam configurations. To find the the upper limit on the total change from non-hadron production effects we add the numbers in quadrature including their uncertainties. For the LE beam we find an upper limit of $2.7 \%$ on the total number of $\nu_{e}$ events and $3.4 \%$ for the LE250 beam. 


\section{Bibliography}

[1] W. Pauli, Dear radioactive ladies and gentlemen, Phys. Today 31N9, 27 (1978).

[2] O. Hahn and L. Meitner, Uber die Absorption der $\beta$-Strahlen einiger Radioelemente, Physikalische Zeitschrift 9, 321 (1908).

[3] O. von Baeyer, O. Hahn, and L. Meitner, Nachweis von $\beta$-Strahlen bei Radium D, Physikalische Zeitschrift 12, 378 (1911).

[4] J. Chadwick, Intensitätsverteilung im magnetischen Spektrum der $\beta$-Strahlen von Radium B+C, Verhandlungen der Deutschen Physikalischen Gesellschaft 16, 383 (1914).

[5] C. D. Ellis and W. A. Wooster, The Average Energy of Disintegration of Radium E, Proceedings of the Royal Society of London A117, 109 (1927).

[6] C. J., The Existence of a Neutron, Proceedings of the Royal Society of London A136, 692 (1929).

[7] E. Fermi, An attempt of a theory of beta radiation. 1, Z. Phys. 88, 161 (1934).

[8] F. L. Wilson, Fermi's Theory of Beta Decay, American Journal of Physics 36, 1150 (1968), English translation of [7].

[9] F. Reines and C. L. Cowan, Detection of the free neutrino, Phys. Rev. 92, 830 (1953). 
[10] C. L. Cowan, F. Reines, F. B. Harrison, H. W. Kruse, and A. D. McGuire, Detection of the free neutrino: A Confirmation, Science 124, 103 (1956).

[11] G. Danby et al., Observation of High-Energy Neutrino Reactions and the Existence of Two Kinds of Neutrinos, Phys. Rev. Lett. 9, 36 (1962).

[12] M. L. Perl et al., Evidence for anomalous lepton production in e+ e- annihilation, Phys. Rev. Lett. 35, 1489 (1975).

[13] Particle Data Group Collaboration: C. Amsler et al., Review of particle physics, Phys. Lett. B667, 1 (2008).

[14] DONUT Collaboration: K. Kodama et al., Observation of tau-neutrino interactions, Phys. Lett. B504, 218 (2001).

[15] S. L. Glashow, Partial Symmetries of Weak Interactions, Nucl. Phys. 22, 579 (1961).

[16] A. Salam and J. C. Ward, Electromagnetic and weak interactions, Phys. Lett. 13, 168 (1964).

[17] S. Weinberg, A Model of Leptons, Phys. Rev. Lett. 19, 1264 (1967).

[18] Gargamelle Neutrino Collaboration: F. J. Hasert et al., Observation of neutrinolike interactions without muon or electron in the Gargamelle neutrino experiment, Phys. Lett. B46, 138 (1973).

[19] M. Gell-Mann and A. Pais, Behavior of neutral particles under charge conjugation, Phys. Rev. 97, 1387 (1955).

[20] A. Pais and O. Piccioni, NOTE ON THE DECAY AND ABSORPTION OF THE THETA0, Phys. Rev. 100, 1487 (1955).

[21] B. Pontecorvo, Inverse beta processes and nonconservation of lepton charge, Sov. Phys. JETP 7, 172 (1958). 
[22] B. Pontecorvo, Neutrino experiments and the question of leptonic-charge conservation, Sov. Phys. JETP 26, 984 (1968).

[23] Z. Maki, M. Nakagawa, and S. Sakata, Remarks on the unified model of elementary particles, Prog. Theor. Phys. 28, 870 (1962).

[24] J. Davis, Raymond, Attempt to detect the antineutrinos from a nuclear reactor by the $\mathrm{Cl}^{37}\left(\bar{\nu}, e^{-}\right) \mathrm{A}^{37}$ reaction, Phys. Rev. 97, 766 (1955).

[25] B. Pontecorvo, Inverse beta process, Camb. Monogr. Part. Phys. Nucl. Phys. Cosmol. 1, 25 (1991).

[26] Davis, Raymond, Jr. and Harmer, Don S. and Hoffman, Kenneth C., Search for neutrinos from the sun, Phys. Rev. Lett. 20, 1205 (1968).

[27] J. N. Bahcall, N. A. Bahcall, and G. Shaviv, Present status of the theoretical predictions for the Cl- 36 solar neutrino experiment, Phys. Rev. Lett. 20, 1209 (1968).

[28] V. N. Gribov and B. Pontecorvo, Neutrino astronomy and lepton charge, Phys. Lett. B28, 493 (1969).

[29] KAMIOKANDE-II Collaboration: K. S. Hirata et al., Observation of B-8 Solar Neutrinos in the Kamiokande-II Detector, Phys. Rev. Lett. 63, 16 (1989).

[30] A. I. Abazov et al., Search for neutrinos from sun using the reaction Ga-71 (electronneutrino e-) Ge-71, Phys. Rev. Lett. 67, 3332 (1991).

[31] GALLEX Collaboration: P. Anselmann et al., Solar neutrinos observed by GALLEX at Gran Sasso., Phys. Lett. B285, 376 (1992).

[32] SNO Collaboration: Q. R. Ahmad et al., Direct evidence for neutrino flavor transformation from neutral-current interactions in the Sudbury Neutrino Observatory, Phys. Rev. Lett. 89, 011301 (2002). 
[33] SNO Collaboration: B. Aharmim et al., Electron energy spectra, fluxes, and daynight asymmetries of B-8 solar neutrinos from the 391-day salt phase SNO data set, Phys. Rev. C72, 055502 (2005).

[34] KamLAND Collaboration: T. Araki et al., Measurement of neutrino oscillation with KamLAND: Evidence of spectral distortion, Phys. Rev. Lett. 94, 081801 (2005).

[35] Super-Kamiokande Collaboration: J. Hosaka et al., Three flavor neutrino oscillation analysis of atmospheric neutrinos in Super-Kamiokande, Phys. Rev. D74, 032002 (2006).

[36] T. J. Haines et al., Calculation of Atmospheric Neutrino Induced Backgrounds in a Nucleon Decay Search, Phys. Rev. Lett. 57, 1986 (1986).

[37] KAMIOKANDE-II Collaboration: K. S. Hirata et al., Experimental Study of the Atmospheric Neutrino Flux, Phys. Lett. B205, 416 (1988).

[38] Frejus Collaboration: C. Berger et al., Study of Atmospheric Neutrino Interactions with the Frejus Detector, Phys. Lett. B227, 489 (1989).

[39] The NUSEX Collaboration: M. Aglietta et al., Experimental study of atmospheric neutrino flux in the NUSEX experiment, Europhys. Lett. 8, 611 (1989).

[40] W. W. M. Allison et al., Measurement of the atmospheric neutrino flavour composition in Soudan-2, Phys. Lett. B391, 491 (1997).

[41] Super-Kamiokande Collaboration: Y. Fukuda et al., Evidence for oscillation of atmospheric neutrinos, Phys. Rev. Lett. 81, 1562 (1998).

[42] Super-Kamiokande Collaboration: Y. Ashie et al., Evidence for an oscillatory signature in atmospheric neutrino oscillation, Phys. Rev. Lett. 93, 101801 (2004). 
[43] K2K Collaboration: M. H. Ahn et al., Measurement of Neutrino Oscillation by the K2K Experiment, Phys. Rev. D74, 072003 (2006).

[44] K. Anderson et al., The NuMI Facility Technical Design Report, FERMILABDESIGN-1998-01.

[45] Z. Pavlovic, Observation of Disappearance of Muon Neutrinos in the NuMI Beam, FERMILAB-THESIS-2008-59.

[46] MINOS Collaboration: D. G. Michael et al., Observation of muon neutrino disappearance with the MINOS detectors and the NuMI neutrino beam, Phys. Rev. D77, 072002 (2008).

[47] Z. Pavlovic, Systematic Uncertainties in the NuMI Flux (2006), MINOS-doc-1283.

[48] MINOS Collaboration: P. Adamson et al., Measurement of Neutrino Oscillations with the MINOS Detectors in the NuMI Beam, Phys. Rev. Lett. 101, 131802 (2008).

[49] MINOS Collaboration: D. G. Michael et al., The Magnetized steel and scintillator calorimeters of the MINOS experiment, Nucl. Instrum. Meth. A596, 190 (2008).

[50] P. V. Vahle, Electromagnetic interactions in the MINOS detectors, FERMILABTHESIS-2004-35.

[51] M. A. Kordosky, Hadronic interactions in the MINOS detectors, FERMILABTHESIS-2004-34.

[52] C. James, All Experimenters Meetings (2007), http://wwwnumi.fnal.gov/MinosAEM/oldAEM.html.

[53] B. J. Rebel, Neutrino induced muons in the MINOS far detector, FERMILABTHESIS-2004-33. 
[54] J. S. Marshall, A study of muon neutrino disappearance with the MINOS detectors and the NuMI neutrino beam, FERMILAB-THESIS-2008-20.

[55] T. H. Osiecki, A search for sterile neutrinos in MINOS, FERMILAB-THESIS2007-77.

[56] A. Ferrari, P. R. Sala, A. Fasso, and J. Ranft, FLUKA: A multi-particle transport code (Program version 2005), CERN-2005-010.

[57] R. Brun et al., GEANT3 manual., CERN Program Library Long Writeup W5013, 1994. CERN Program Library Long Writeup W5013.

[58] H. Gallagher, The NEUGEN neutrino event generator, Nucl. Phys. Proc. Suppl. 112, 188 (2002).

[59] C. Zeitnitz and T. A. Gabriel, The GEANT - CALOR interface and benchmark calculations of ZEUS test calorimeters, Nucl. Instrum. Meth. A349, 106 (1994).

[60] D. Rein and L. M. Sehgal, Neutrino Excitation of Baryon Resonances and Single Pion Production, Ann. Phys. 133, 79 (1981).

[61] NuTeV Collaboration: G. P. Zeller et al., A precise determination of electroweak parameters in neutrino nucleon scattering, Phys. Rev. Lett. 88, 091802 (2002).

[62] R. C. Allen et al., Study of electron-neutrino electron elastic scattering at LAMPF, Phys. Rev. D47, 11 (1993).

[63] NOMAD Collaboration: P. Astier et al., Prediction of neutrino fluxes in the NOMAD experiment, Nucl. Instrum. Meth. A515, 800 (2003).

[64] L. A. Ahrens et al., DETERMINATION OF THE NEUTRINO FLUXES IN THE BROOKHAVEN WIDE BAND BEAMS, Phys. Rev. D34, 75 (1986).

[65] H. W. Atherton et al., PRECISE MEASUREMENTS OF PARTICLE PRODUCTION BY 400-GeV/c PROTONS ON BERYLLIUM TARGETS, CERN-80-07. 
[66] NA56/SPY Collaboration: A. Marchionni, Measurement of charged particle production from 450-GeV/c protons on beryllium, Nucl. Phys. Proc. Suppl. 75A, 194 (1999).

[67] D. S. Barton et al., Experimental Study of the a-Dependence of Inclusive Hadron Fragmentation, Phys. Rev. D27, 2580 (1983).

[68] N. V. Mokhov, The MARS code system user's guide version 13(95), FERMILABFN-0628.

[69] M. Bonesini, A. Marchionni, F. Pietropaolo, and T. Tabarelli de Fatis, On particle production for high energy neutrino beams, Eur. Phys. J. C20, 13 (2001).

[70] A. J. Malensek, Empirical Formula for Thick Target Particle Production, FERMILAB-FN-0341.

[71] MIPP Collaboration: H. Meyer, Physics of the MIPP experiment, Nucl. Phys. Proc. Suppl. 142, 453 (2005).

[72] S. M. Seun, Measurement of $\pi-K$ Ratios from the NuMI Target, FERMILABTHESIS-2007-61.

[73] R. P. Feynman, Very high-energy collisions of hadrons, Phys. Rev. Lett. 23, 1415 (1969).

[74] F. James and M. Roos, Minuit: A System for Function Minimization and Analysis of the Parameter Errors and Correlations, Comput. Phys. Commun. 10, 343 (1975).

[75] R. Brun and F. Rademakers, ROOT: An object oriented data analysis framework, Nucl. Instrum. Meth. A389, 81 (1997).

[76] M. G. Catanesi et al., Proposal for hadron production measurements using the NA49 detector for use in long-baseline and atmospheric neutrino flux calculations, CERN-SPSC-2001-017. 
[77] J. J. Evans, Measuring Antineutrino Oscillations with the MINOS Experiment, FERMILAB-THESIS-2009-14.

[78] R. Ospanov, A measurement of muon neutrino disappearance with the MINOS detectors and NuMI beam, FERMILAB-THESIS-2008-04.

[79] A. Blake, A likelihood method for CC/NC separation (2007), MINOS-doc-2720.

[80] MINOS Collaboration: D. G. Michael et al., Observation of muon neutrino disappearance with the MINOS detectors and the NuMI neutrino beam.

[81] The MINOS Collaboration, Preliminary Results from MINOS on $\nu_{\mu}$ Disappearance Based on an Exposure of $2.5 \times 10^{20} 120 \mathrm{GeV}$ Protons on the NuMI Target, (2007), Contributed to the XXIII International Symposium on Lepton and Photon Interactions at High Energy, Daegu, Korea, 13-18 August 2007.

[82] N. Salidou, ANN for CC- NC Separation (2007), MINOS-doc-2735.

[83] B. Armstrong, CC PID Task Force Interim Report (2008), MINOS-doc-4019.

[84] A. Hocker et al., TMVA: Toolkit for multivariate data analysis, PoS ACAT, 040 (2007).

[85] T. Mann, Bottom-Fishing for QE-like Events below the Oscillation Minimum Open season (2007), MINOS-doc-3333.

[86] R. Milburn, Systematic Error from Residual Geomagnetic Field in the NuMI Beam Pipe (2007), MINOS-doc-4633.

[87] J. Hylen, private communication 2008.

[88] M. Dorman et al., Observation of Changes to the NuMI Beamline Status with Near Detector Data (2008), MINOS-doc-4496.

[89] M. Bishai et al., Update on NumI Target Damage Study (2008), MINOS-doc-5081. 
[90] J. Paley, CC Handscan Results (2009), MINOS-doc-5613.

[91] J. Marshall, A Data-Driven Correction to the MC NC Background (2007), MINOSdoc-3307.

[92] R. Hatcher et al., Range Curvature Task Force Position Paper (2007), MINOSdoc-3134.

[93] N. Devenish and J. Hartnell, Unblinding of Run III ¿4 GeV region (2008), MINOSdoc-4912.

[94] V. D. Barger, J. G. Learned, S. Pakvasa, and T. J. Weiler, Neutrino decay as an explanation of atmospheric neutrino observations, Phys. Rev. Lett. 82, 2640 (1999).

[95] G. L. Fogli, E. Lisi, A. Marrone, and D. Montanino, Status of atmospheric neutrino(mu)i-¿neutrino(tau) oscillations and decoherence after the first K2K spectral data, Phys. Rev. D67, 093006 (2003).

[96] D. Colladay and V. A. Kostelecky, CPT violation and the standard model, Phys. Rev. D55, 6760 (1997).

[97] D. Colladay and V. A. Kostelecky, Lorentz-violating extension of the standard model, Phys. Rev. D58, 116002 (1998).

[98] G. J. Feldman and R. D. Cousins, A Unified Approach to the Classical Statistical Analysis of Small Signals, Phys. Rev. D57, 3873 (1998).

[99] PBEAM by Noel Stanton, see P-875: A Long-baseline Neutrino Oscillation Experiment at Fermilab, 33 (1995) for a description.

[100] L. Loiacono, Beam Based Alignment of the NuMI Horns Target and Baffle (2008), MINOS-doc-4828.

[101] J. Hylen, Calibration of NuMI Horn Current Readout (2006), MINOS-doc-1303. 